\title{
TRAVTEK EVALUATION TASK C3 - CAMERA CAR STUDY
}

\section{NOTE TO READER:}

\section{THIS IS A LARGE DOCUMENT}

Due to its large size, this document has been segmented into multiple files. All files separate from this main document file are accessible from links (blue type) in the table of contents or the body of the document. 


\section{TravTek Evaluation Task $\mathrm{C}_{3}$ Camera Car Study}

Publication No. FHWA-RD-94-076

June 1995

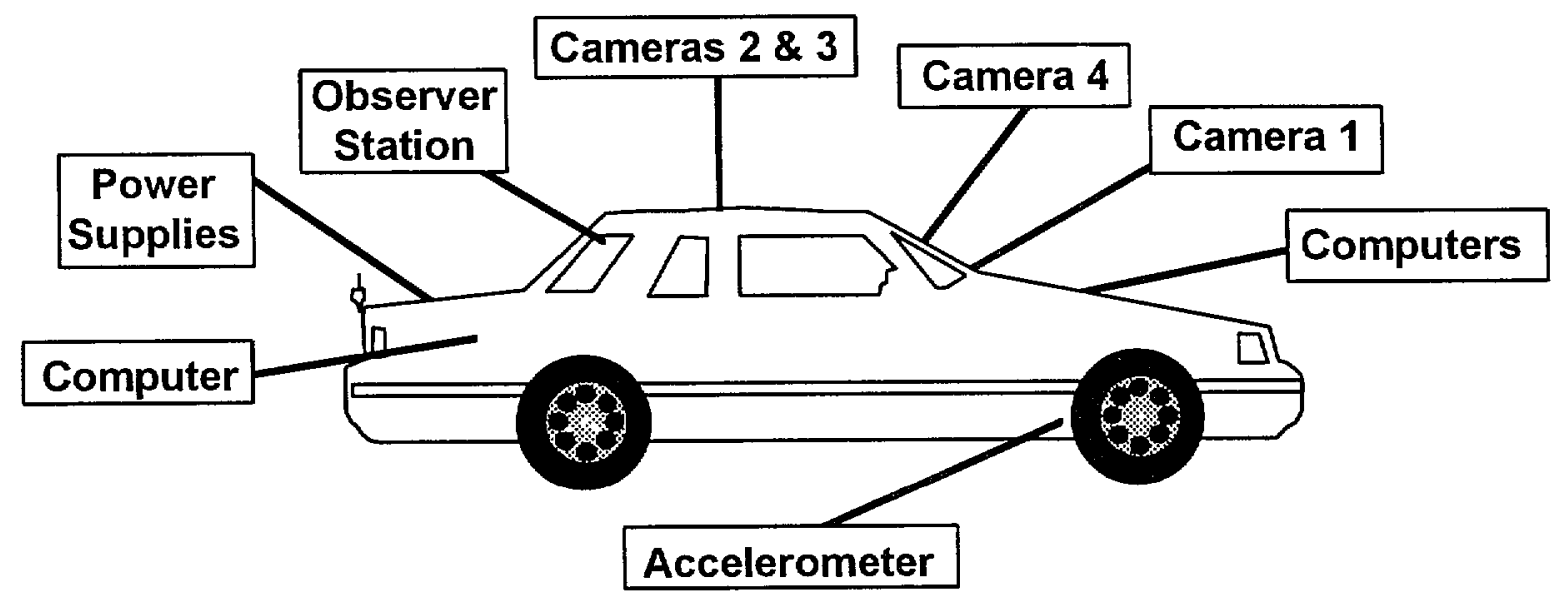

Research and Development

Turner-Fairbank Highway Research Center

US. Department of Transportation 6300 Georgetown Pike

Federal Highway Administration 


\section{FOREWORD}

This report is one of eight reports produced as part of the evaluation of the Travtek operational field test, conducted in Orlando, Florida, during 1992-1993. Travtek, short for Travel Technology, was an advanced driver information and traffic management system that provided a combination of traveler information services and route navigation and guidance support to the driver. Twelve individual but related studies were conducted during the evaluation. Evaluation goals and objectives were represented by the following basic questions: (1) Did the TravTek system work? (2) Did drivers save time and avoid congestion? (3) Will drivers use the system?- (4) How effective was voice guidance compared to moving map and turn-by-turn displays? (5) Was TravTek safe? (6) Could TravTek benefit travelers who do not have the TravTek system? (7) Will people be willing to pay for TravTek features?

Evaluation data were obtained from more than 4,000 volunteer drivers during the operation of 100 specially equipped automobiles for a l-year period. Results of the evaluation demonstrated and validated the concept of in-vehicle navigation and the provision of traveler information services to the driver. The test also provided valuable results concerning the drivers'interaction with and use of the in-vehicle displays. This project has made many important contributions supporting the goals and objectives of the Intelligent Transportation Systems Program.

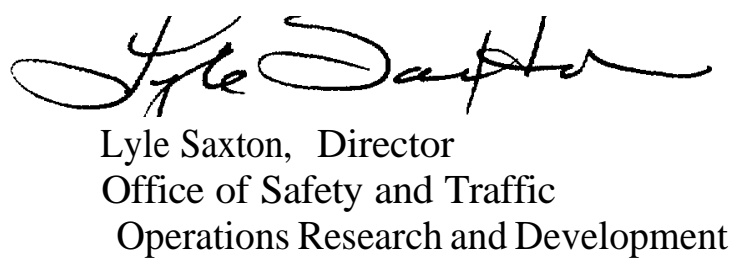

\section{NOTICE}

This document is disseminated under the sponsorship of the Department of Transportation in the interest of information exchange. The United States Government assumes no liability for the contents or the use thereof. This report does not constitute a standard, specification, or regulation.

The United States Government does not endorse products of manufacturers. Trade and manufacturers' names appear in this report only because they are considered essential to the object of the document. 


\begin{tabular}{|c|c|c|}
\hline $\begin{array}{l}1 \text { Report No. } \\
\text { FHWA-RD-94-076 }\end{array}$ & 2. Government Accession №. & 3. Recipient's Catalog No. \\
\hline \multicolumn{2}{|l|}{ 4. Title and Subtitle } & 5. ReportDate June 1995 \\
\hline \multicolumn{2}{|c|}{ TRAVTEK EVALUATION TASK C3 - CAMERA CAR STUDY } & 6. Performing Organization Code \\
\hline \multicolumn{2}{|c|}{$\begin{array}{l}\text { 7. Author(s) T. Dingus, D. McGehee, M. Hulse, S. Jahns, N. Manakkal, } \\
\text { M. Mollenbauer, \& R. Fleischman }\end{array}$} & 8. Performing Organization Report No. \\
\hline \multirow{2}{*}{\multicolumn{2}{|c|}{$\begin{array}{l}\text { 9. Performing Organization Name and Address } \\
\text { Performance and Safety Sciences, Inc. } \\
29 \text { Forest Hill Place NE } \\
\text { Iowa City, IA } 52240\end{array}$}} & $\begin{array}{l}\text { 10. Work Unit No. (TRAIS) } \\
\text { 3B7A }\end{array}$ \\
\hline & & $\begin{array}{l}\text { 11. Contractor Grant No. } \\
\text { DTFH61-91-C-00106 }\end{array}$ \\
\hline \multirow{2}{*}{\multicolumn{2}{|c|}{$\begin{array}{l}\text { 12. Sponsoring Agency Name and Address } \\
\text { Office of Safety and Traffic Operations R\&D } \\
\text { Federal Highway Administration } \\
6300 \text { Georgetown Pike } \\
\text { McLean, VA } 22 \text { 102-2296 }\end{array}$}} & $\begin{array}{l}\text { 13. Type of Report and Period Covered } \\
\text { Final Report/ } 9-92 \text { to } 5-94\end{array}$ \\
\hline & & 14. Sponsoring Agency Code \\
\hline
\end{tabular}

15. Supplementary Notes

Contracting Officer's Technical Representative (COTR) Frank Mammano HSR-I2

16. Abstract

The goal of the TravTek Camera Car Study was to furnish a detailed evaluation of driving and navigation performance, system usability, and safety for the TravTek system. To achieve this goal, an instrumented "camera car" was developed to provide comprehensive driving performance and behavior measurement capability. Six navigation test configurations were evaluated in the camera car study. These included:

- TravTek route-map display.

- TravTek route-map display with supplementary voice guidance.

- TravTek symbolic guidance-map display.

- TravTek symbolic guidance-map display with supplementary voice guidance.

- Paper map.

- Paper textual direction list.

A primary finding of this research was that turn-by-turn guidance information (whether presented verbally, in a textual list or by a graphic display) enhances the performance, usability, and/or safety when compared with alternatives which provide holistic route information. For this study, the TravTek turn-by-turn with voice condition and a paper direction list (with a large legible font and similar in layout to a computer generated list found at some rental-car counters) provided the best overall performance. The TravTek turn-by turn without voice and route-map with voice conditions were comparable in many respects to these conditions, but did not perform as well with respect to driving performance and safety-related driver error. In contrast, the TravTek route-map without voice had the greatest overall impact on the driving task and was the least safe of all the navigation conditions tested. However, these safety differences are mitigated by user experience, and by driver selection of other available options (as shown in other TravTek studies). The paper map control condition was the least usable means of navigation in the study and resulted in substantially worse navigation performance than any other condition.

\begin{tabular}{|l|l|l|l|}
\hline $\begin{array}{l}\text { 17. Key Words } \\
\text { TravTek, ATIS, Navigation, Camera Car }\end{array}$ & $\begin{array}{l}\text { 18. Distribution Statement } \\
\text { No restrictions. This document is available to the public through } \\
\text { the National Technical Information Service, Springfield, Virginia } \\
22161\end{array}$ \\
\hline $\begin{array}{l}\text { 19. Security Classif. (of this report) } \\
\text { Unclassified }\end{array}$ & $\begin{array}{l}\text { 20. Security Classif. (of this page) } \\
\text { Unclassified }\end{array}$ & $\begin{array}{l}\text { 21. No. of Pages } \\
261\end{array}$ & \begin{tabular}{l} 
22. Price \\
\hline
\end{tabular}
\end{tabular}

Form DOT F $1700.7 \quad(8-72) \quad$ Reproduction of completed page authorized 


\section{PREFACE}

The TravTek system constitutes a major Intelligent Vehicle-Highway System (IVHS) Advanced Traveler Information System (ATIS) demonstration project. The system provided in-vehicle information via color touchscreen CRT, steering wheel buttons, and synthesized voice. The TravTek driver interface was developed with the intent of providing navigation, services and attractions, and roadway incident and traffic information to the driver. The design of the TravTek interface had as its primary objectives: (1) more effective driver navigation providing the benefit of saving time, (2) easy access to valuable and convenient location information to alleviate stress and increase driving enjoyment, (3) maintenance of safe driving performance during system use and safety improvement facilitated by information for avoiding potential hazards and for emergency response, and (4) improvement of roadway efficiency to alleviate congestion.

The final report provides detailed data regarding driver performance and behavioral interactions with four navigation configurations. In addition, these configurations were also compared to two conventional methods of navigation: a paper map and a textual direction list. Human factors issues addressed as part of this final report include: (1) Which navigation configuration(s) result in the best driving performance?, (2) Which navigation configuration(s) result(s) in the best navigation performance?, (3) Can any of the TravTek configuration designs be improved, (4) Do any of the navigation configurations result in unsafe driving behavior?, (5) Do driving performance, navigation performance, and driving safety vary as a function of TravTek experience?, (6) Do driving performance, navigation performance, and driving safety vary as a function of age?, and (7) Do driving performance, navigation performance, and driving safety vary as a function of driver are familiarity? 


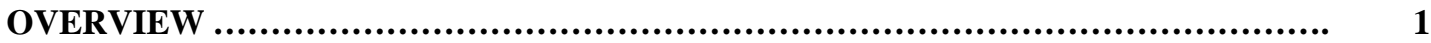

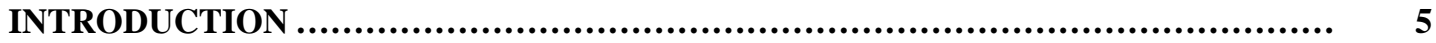

BACKGROUND . ............................................................... 5

Study Rationale ......................................................... 5

Relevant Literature And Past Research .................................. 6

Etak Navigator Studies ............................................... 6

Additional Navigation System Studies ............................. 6

Preliminary TravTek Research ............................................. 6

PURPOSE OF TEST ............................................................. 7

OBJECTIVES ......................................................................... 7

ISSUE 1: WHICH NAVIGATION CONFIGURATION(S)

RESULTED IN THE LEAST DRIVING TASK INTRUSION? ............. 9

Objectives ................................................................. 10

Hypotheses ................................................................ 11

Measures of Effectiveness ..................................................... 11

Measures of Performance ................................................... 11

Number and Duration of Lane Deviations .......................... 11

Steering Wheel Position Variance and Number of Large Steering

Reversals ........................................................ 12

Average Vehicle Velocity and Velocity Variance ............................. 12

Lateral Acceleration Measures ...................................................... 12

Longitudinal Acceleration Measures and Braking Data.................. 12

Time Spent Scanning the Roadway Environment and Navigation

Aids

ISSUE 2: WHICH NAVIGATION CONFIGURATION(S) RESULTED IN THE

BEST NAVIGATION PERFORMANCE?........................................... 13

Objectives ............................................................................................................. 13

Hypotheses ......................................................................................................................... 14

Measures of Effectiveness ................................................................................................ 14

Measures of Performance ................................................................................................ 14

Navigation Performance ....................................................................... 14

Driver Perception of Configuration Contribution to Navigation

Performance ..................................................................................... 15

ISSUE 3: COULD ANY OF THE TRAVTEK CONFIGURATION DESIGNS BE

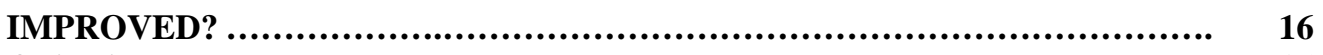

Objectives ............................................................................. 16

Hypotheses ....................................................................... 17

Measures of Effectiveness .......................................................... 17

Measures of Performance .............................................................. 17

Maneuver Timing/Abruptness. ........................................... 17

Number and Duration of Glances to the Navigation System .................. 17

Experimenter Observation .................................................. 17

ISSUE 4: DID ANY OF THE NAVIGATION CONFIGURATIONS RESULT IN UNSAFE

DRIVING BEHAVIOR?. ........................................................ 18

Objectives ......................................................................... 18

Hypotheses ............................................................................ 19

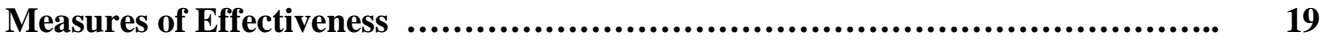

Measures of Performance ............................................................. 21

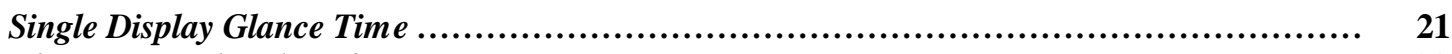

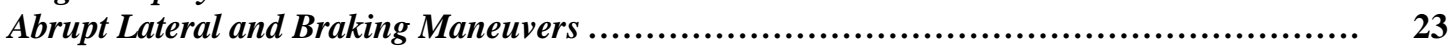


TABLE OF CONTENTS (Continued)

Section

$\underline{\text { Page }}$

Unplanned Lane Deviations and Turning Track Errors .......... 23

Late/inappropriate Reaction to an External Event ............... 24

Unplanned Speed Variation in Excess of 16 km/h (IO mi/h)....... 24

Stopping Or Slowing in Unsafe Circumstances ............................... 24

Unsafe Intersection Behavior.. ................................................... 24

Dangerously Close Headways .................................................... 24

Mental Workload..................................................................... 24

ISSUE 5: DID DRIVING PERFORMANCE, NAVIGATION

PERFORMANCE, AND DRIVING SAFETY VARY AS A FUNCTION

OF TRAVTEK EXPERIENCE .............................................. 25

ISSUE 6: DID DRIVING PERFORMANCE, NAVIGATION

PERFORMANCE, AND DRIVING SAFETY VARY AS A

FUNCTION OF AGE? .................................................... 25

ISSUE 7: DID DRIVING PERFORMANCE, NAVIGATION

PERFORMANCE, AND DRIVING SAFETY VARY AS A

FUNCTION OF TYPE OF USER

(I.E. LOCAL OR VISITOR) AND AREA FAMILIARITY?.............. 25

METHODS

(I.E. LOCAL OR VISITOR) AND AREA FAMLI....................................................................... 27

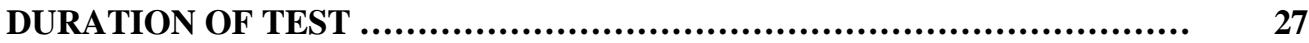

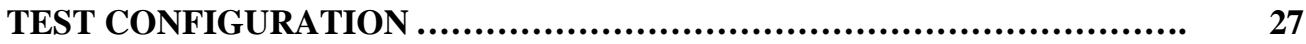

Symbolic Guidance Display .............................................. 27

Route Map Display. ........................................................ 28

Aural Display Configurations ................................................ 29

TEST CONDITIONS. ................................................................ 30

Origin/Destination Pairs ..................................................... 30

Conducting Tests Using Origin/Destination Pairs ....................... 31

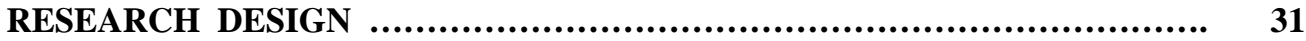

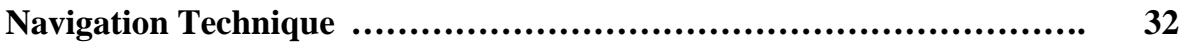

Paper Map and Textual Direction List ........................... 32

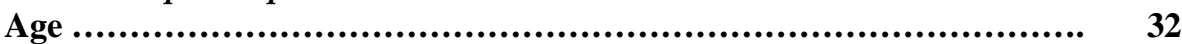

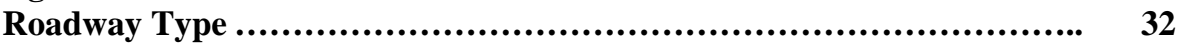

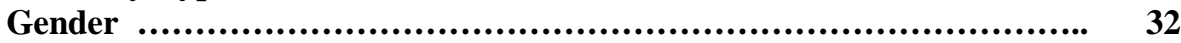

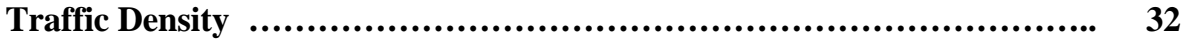

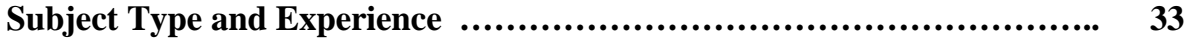

Training Tools ................................................................... 33

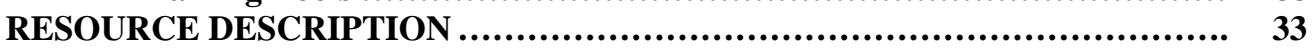

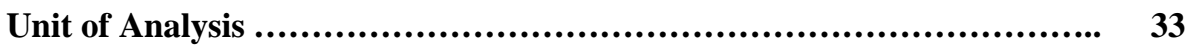

Camera Car Equipment Description ........................................... 33

Eye Glance Camera ............................................ 35

Forward View Camera ............................................. 36

Vehicle Information Center/Steering Wheel Camera ................. 36

Lane Tracking Camera ................................................. 37

Lateral and Longitudinal Accelerometer ............................ 37

Data Collection Computer........................................... 37

Experimenter Control Panel .................................... 37

Video/Sensor/Experimenter Control Panel Interface.. ...................... . 38

Audio Data Collection System ................................... 38

Multiplexer ...................................................... 38

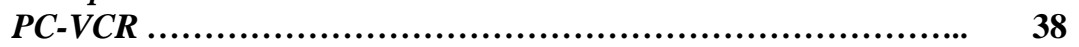


Ideas and Suggestions for Future Systems ............................. 84

Conclusions on 0 verall Safety and U sability................................... 85

ISSUE 4: DO ANY OF THE NAVIGATION CONFIGURATIONS

RESULT IN UNSAFE DRIVING BEHAVIOR“............................. 85

Operational Definitions.. ......................................................... 86

0 perational Definitions for Potential Severity Categories................. 86

O perational Definitions for Environmental Proximity Categories......... 87

O perational D efinitions for Navigation Condition as a Causal Factor .. 88

Measures Of Performance For Safety .......................................... 88

Number of Accidents .................................................... . 88

N umber of $\mathrm{Near}$ Misses ................................................... 88

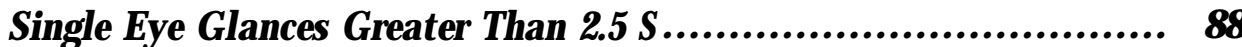

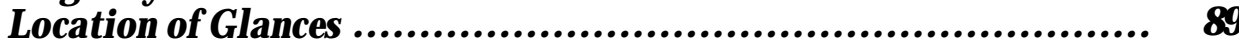

Abrupt Lateral Accelerations and Braking Maneuvers.................. 89

Unplanned Lane Deviations ............................................... 89

Reaction to External Events................................................ 89

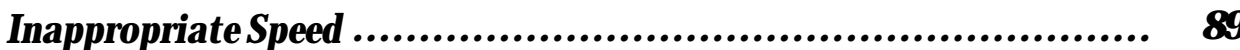

Stopping in U nsafe Circumstances ................................... 90

Subjective W orkload Ratings for O verload .............................. 90

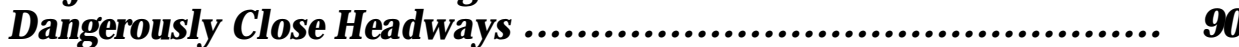

Interpretation Of The Camera Car Safety Analysis Results ........... 90

Measures Of Performance For Near Misses And Unsafe Acts ...... . 92

N umber of Accidents ................................................... 92

Number of Near Misses, Plus 0 ther Measures of Environmental

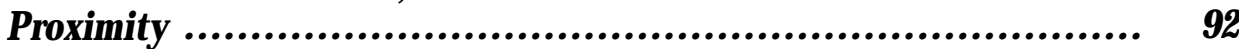

N umber and Potential Severity of Incidents ............................. 94

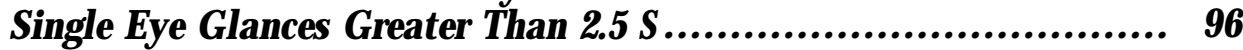

Abrupt Lateral Accelerations............................................. 97

Abrupt Braking Maneuvers .............................................. 98

Unplanned Lane Deviations .......................................... 98

Reaction to External Events............................................. 99

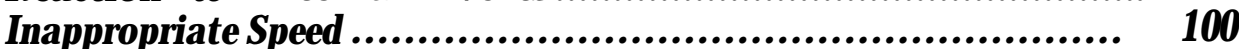

Stopped Vehicle in Unsafe Circumstances ............................. 101

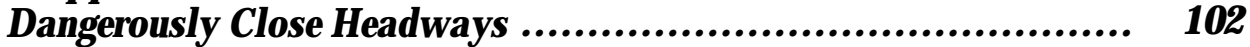

SubjectiveW orkload Ratings for O verl oad ............................ 103

General Risk Assessment Analysis ......................................... 104

Discussion.. ............................................................... 106

ISSUE 5: DO DRIVING PERFORMANCE, NAVIGATION

PERFORMANCE, AND DRIVING SAFETY VARY AS A

FUNCTION OF TRAVTEK EXPERIENCE ...................................... 108

Navigation Condition Usage Between Experience Levels............ 108

Measures Of Performance For Driving Performance .................. 109

Driver Eye Glance Behavior ........................................... 109

Mean Speed ........................................................... 109

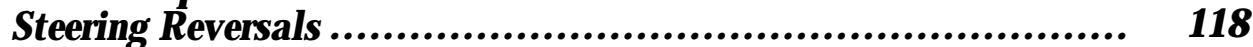

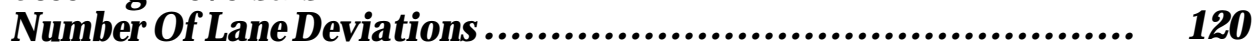

Brake Applications...................................................... 120

Performance Measure Discussion ......................................... 121

Measures of Performance For Navigation Performance.............. 122

Time Taken to Plan a Trip ............................................. 122

Total Time Taken to Plan and D rive to the Destination .................. 122 
Amount of Time the Vehicle Was Stopped En R oute to the

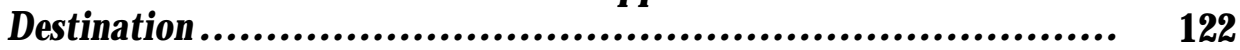

Navigation Condition Main Effects ................................. 125

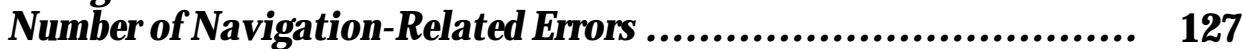

Discussion For Navigation Performance .............................. 127

Additional Measures of Performance Indicative of Ease of Use . . 128 Workload .............................................................. 128 Location Of Glances .................................................. 132

Discussion For Usability .................................................... 133

Measures of Performance for Near Misses and Unsafe Acts....... 137 N umber of Safety-Related Errors .................................... 138 Number of Navigation Glances G reater Than $2.5 \mathrm{~S} \ldots \ldots \ldots \ldots \ldots \ldots \ldots . . . . . . .138$

Discussion of Near Misses and Unsafe Acts ......................... 138 ISSUE 6: DO DRIVING PERFORMANCE, NAVIGATION PERFORMANCE, AND DRIVING SAFETY VARY AS A FUNCTION OF AGE .................................................................. 140

Measures of Performance for Driving Performance ................... 140 Driver Eye Glance Behavior ........................................... 140 Variance in Lateral Acceleration ..................................... 144 Variance in Longitudinal Acceleration ................................. 145 Negative Longitudinal Acceleration ................................... 145 Variance in Negative Longitudinal Acceleration ........................... 145 M ean Speed ............................................................. 145

N umber of Steering Wheel Reversals Corrected for Trip Time ......... 146

Discussion of Performance Measures..................................... 147

Measures for Navigation Performance .................................... 148

Time Required to Plan a Trip .......................................... 148

Time Required to Drive to a Destination ............................., 148 Total Time Required to Plan and Drive to a Destination................. 149 Navigation Error Data ................................................., 150

Discussion For Navigation Performance ............................. 152

Additional Measures of Performance Indicative of Ease of Use . . 152 Location of Glances ................................................. 153

Discussion For Usability .................................................... 154 Eye Glance Location .................................................... 154

Measures Of Performance For Near Misses And Unsafe Acts.. ... 173 N umber of Safety-Related Errors ...................................... 173

Safety Discussion.............................................................. 174

General Discussion of Age Effects ....................................... 175

ISSUE 7: DO DRIVING PERFORMANCE, NAVIGATION

PERFORMANCE, AND DRIVING SAFETY VARY AS A

FUNCTION OF DRIVER AREA FAMILIARITY3........................... 176

Measures of Performance for Driving Performance ................. 176

Driver Eye Glance Behavior ........................................ 182

Variance in Lateral Acceleration .................................... 182

Performance Measure Discussion .......................................... 183

Measures of Performance for Navigation Performance................. 185

Time Required to Plan a Trip........................................... 187

Number of Navigation-R elated Errors. ..............................., 187

Discussion For Navigation Performance ............................... 188

Additional Measures of Performance Indicative of Ease of Use . . 190 
$\underline{\text { Section }}$

Measures of Performance for Near Misses and Unsafe Acts . . . . . 190

Total N umber of Safety-R elated Errors. ...................., 190

N umber and Duration of Lane Excursions.............. . . . . . 192

Discussion of Safety-Related Errors for Area Familiarity... . . . . . . 192

General Discussion of Area Familiarity................... 192

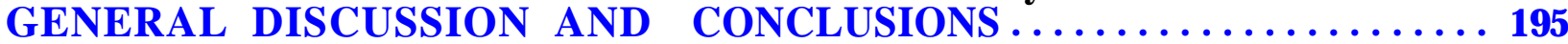

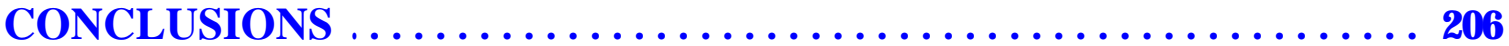

APPENDIX A: INFORMED CONSENT TO PARTICIPATE IN TRAVTEK -

LOCAL USERS STUDY ......................................... 209

APPENDIX B: INFORMED CONSENT TO PARTICIPATE IN TRAVTEK -

VISITORS， STUDY ....................................................... 211

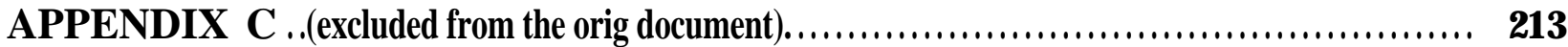

APPENDIX D: DETAILED ERROR NUMBER DESCRIPTION AND

ASSIGNED POTENTIAL SEVERITY .......................... 221

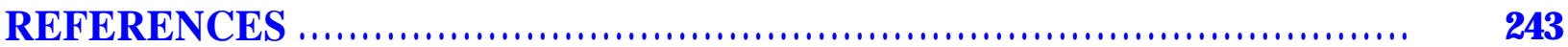




\section{LIST OF FIGURES}

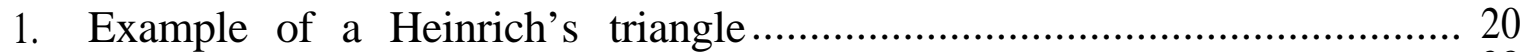

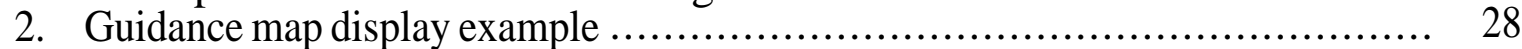

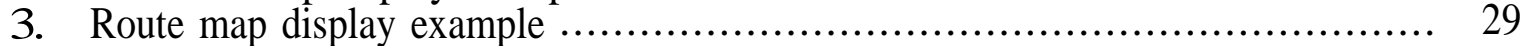

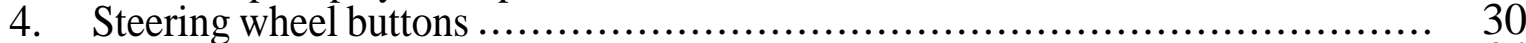

5. Camera car schematic diagram ............................................... 34

6. Camera car photograph of the TravTek display, controls, and selected cameras …. 35

7 Camera car photograph showing the position of the lane tracking camera.............. 36

8. Camera car truck mounted equipment .......................................... 37

9. Camera car experimenter station in the rear seat ................................ 38

10. Glance probabilities for turn-by-turn with voice navigation condition. .............. 48

11. Glance probabilities for turn-by-turn without voice navigation condition. ............ 49

12. Glance probabilities for route map with voice navigation condition ................. 50

13. Glance probabilities for route map without voice navigation condition. ............. 51

14. Glance probabilities for paper directions navigation condition ..................... 52

15. Glance probabilities for paper map navigation condition. …...................... 53

16. Number of glances to each glance location and each navigation condition; all possible locations included ....................................................... 55

17. Duration of glances for each navigation condition; all glance locations included. ..... 57

18. Duration of glances for each navigation condition; only glances to navigation display included..................................................................... 58

19. Duration of glances for each navigation condition; only glances to navigation display included .................................................................... 58

20. Number of glances to the navigation display shown by varying glance durations..... 59

21. Number of glances for each glance location and each navigation condition; only glances occurring in intersections included.

22. Number of glances for each navigation condition; to the navigation display lasting

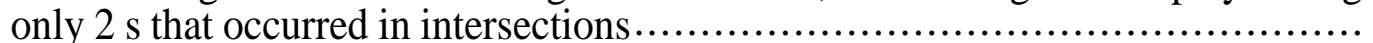

23. Number of glances for each glance location and each navigation condition; only

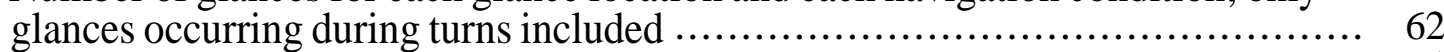

24. Number of lane deviations for each navigation condition .......................... 65

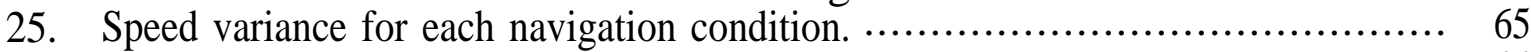

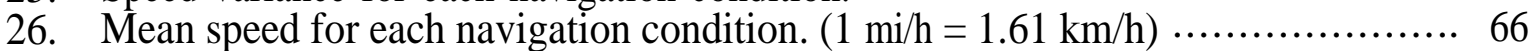

27. Number of abrupt longitudinal acceleration maneuvers for each navigation condition. ..................................................................... 67

28. Total of all three subjective workload rating categories for each navigation

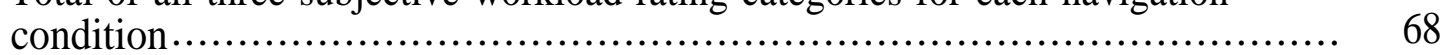

29. Subjective workload ratings of psychological stress for each navigation

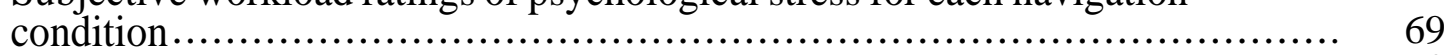

30. Subjective workload ratings of time stress for each navigation condition. ......... 69

31. Subjective workload ratings of visual effort for each navigation condition.......... 70

32. Time required to plan a trip for each navigation condition .......................... 73

33. Time required to drive to a destination for each navigation condition. ............... 73

34. Total time required to plan and drive to the destination for each navigation condition ..................................................................... 74

35. Number of stops made for each navigation condition ............................ 74

36. Amount of time the vehicle was stopped while driving to a destination for each navigation condition..................................................................... 75

37. Number of navigation errors for each navigation condition. ....................... 77

38. Time to plan a trip for each navigation condition. …............................. 78

39. Time required to drive to a destination for each navigation condition. ............... 78 
40. Duration of glances for each navigation condition; only glances at navigation

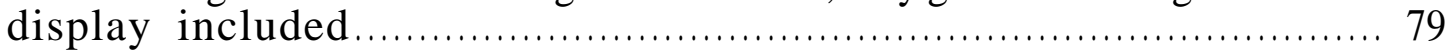

41. Number of glances to the navigation display for each navigation condition........ 80

42. Amount of time the vehicle was stopped while en route to a destination for each

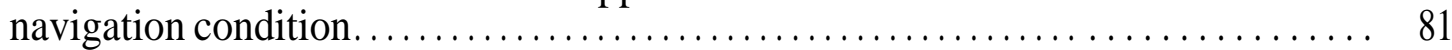

43. Average number of stops made during drive to a destination............ 81

44. Total number of safety-related errors committed by local users; shown by individual subjects....

45. Total number of safety-related errors committed for visitors; shown by individual subjects.

46. Number of safety-related errors for each navigation condition and each environmental proximity; all data included ...

47. Number of safety-related errors caused by a glance at the navigation display for each navigation condition and each environmental proximity; all data included........93

48. Number of safety-related errors for each navigation condition and each potential severity category; all data included

49. Number of safety-related errors for each navigation condition; no hazard present and minor severity incidents excluded

50. Number of incidents in each safety-related error category for each navigation condition; all data included

51. Number of incidents in each safety-related error category where attention was directed at the navigation display. Shown across each navigation condition. . . . . . . 96

52. Number of safety-related errors that exceed designated safe lateral acceleration rate for each navigation condition; all data included

53. Number of safety-related errors that involved braking rates which exceed designated safe deceleration rates for each navigation condition................. 98

54. Total number of lane deviations for each navigation condition; shown for all glance locations and for deviations caused by a glance to the navigation display.. . . . 99

55. Total number of incidents which involved appropriate and inappropriate reaction to external events

56. Total number of slow speed incidents committed within each navigation condition.

57. Total number of stops in the roadway or other unsafe location where the user needed to gather navigation information. Shown for each navigation condition... . 101

58. Total number of unsafe or extended stops at intersections where the user needed to gather navigation information. Shown for each navigation condition. .......... 102

59. Total number of unsafe headway incidents committed within each navigation condition.

60. Total number of occurrences where all three classifications of subjective workload were rated as high. Shown for each navigation condition...................... 104

61. Risk assessment matrix for the safety analysis; determines the risk associated with all combinations of the probable severity of an accident and the environmental proximity at which each safety related event occurred........................ 105

62. Total count of all final hazard risk assessment values computed for each safetyrelated error. Shown by level of risk and each navigation condition.............. 106

63. Duration of glances to the navigation information compared across local users'

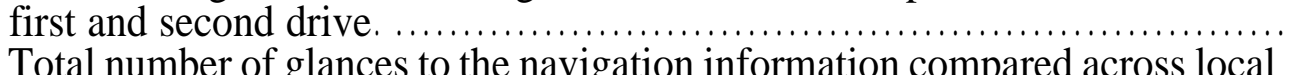

64. Total number of glances to the navigation information compared across local

65. Total number of roadway glances compared across local users' first and second drive 
66. Mean speed for each navigation condition compared across local users' first and second driver.

67. Number of steering reversals over 6 degrees shown per second; compared for local users' first and second drive .............................................

68. Number of steering reversals over 6 degrees shown per second; compared for each navigation condition and corrected for trip time.

69. Number of lane deviations for each age group compared across local users' first and second drive.

70. Number of lane deviations for each navigation condition compared across local users' first and second drive.

71. Number of brake applications shown per second; compared for each navigation condition and local users' first and second rive. Numbers are corrected for trip time.

72. Time required to plan a trip compared across local users' first and second drive ... 126

73. Total time required to plan and drive to the destination compared across local users' first and second drive.

74. Amount of time the vehicle was stopped while en route to the destination; compared for each navigation condition and local users' first and second drives.... 127

75. Number of navigation-related errors by type compared across local users' first and second drive.

76. Subjective workload ratings of time stress for each navigation condition compared across local users' first and second drive.

77. Subjective workload ratings of visual effort for local users' first and second drive. 132

78. Total of all three subjective workload rating categories for each navigation condition compared across local users' first and second drive. ................... 132

79. Turn-by-turn without voice link diagram for local users' first drive. .............. 134 Turn-by-turn without voice link diagram for local users' second drive ............ 135

81. Route map with voice link diagram for local users' first drive .................... 136

82. Route map with voice link diagram for local users' second drive. . ................ 137

83. Number of safety-related errors compared across age and local users' first and

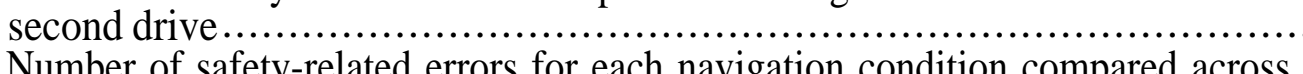

84. Number of safety-related errors for each navigation condition compared across local users' first and second drive....

85. Number of glances greater than $2.5 \mathrm{~s}$ to the navigation display compared across local users' first and second drive

86. Average duration of a glance to the navigation display for each navigation

condition compared across all three age groups

87. Number of glances to the navigation display for each navigation condition compared across the three age groups .................................................. 144

88. Total number of roadway glances compared across the three age groups. .......... 144

89. Variance in lateral acceleration compared across all three age groups. ................ 145

90. Variance in longitudinal acceleration compared across the three age groups. ....... 146

91. Mean negative longitudinal acceleration compared across the three age groups ...... 146

92. Variance in negative longitudinal acceleration compared across the three age

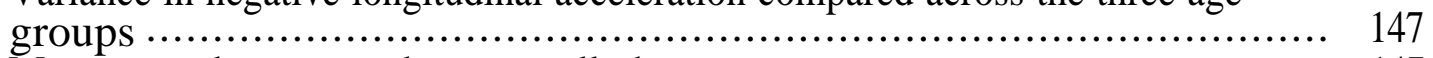

93. Mean speed-compared across all three age groups..

94. Number of steering wheel reversals over 6 degrees shown per second; compared across the three age groups and corrected for trip time............................... 148

95. Time required to plan a trip compared across all three age groups..................... 150

96. Time required to plan a trip shown for each navigation condition compared across the three age groups 
97. Time required to drive to a destination compared across the three age groups, ..... 151

98. Time taken to plan and drive to a destination compared across the three age groups …............................................................. 151

99. Number of navigation-related errors compared across the three age groups. ....... 152

100. Link diagram for turn-by-turn with voice, young age group .......................... 155

101. Link diagram for turn-by-turn with voice, middle age group ..................... 156

102. Link diagram for turn-by-turn with voice, older age group .................... 157

103. Link diagram for turn-by-turn without voice, young age group. .................. 158

104. Link diagram for turn-by-turn without voice, middle age group....................... 159

105. Link diagram for turn-by-turn without voice, older age group........................ 160

106. Link diagram for route map with voice, young age group ............................... 161

107. Link diagram for route map with voice, middle age group...................... 162

108. Link diagram for route map with voice, older age group .......................... 163

109. Link diagram for route map without voice, young age group..................... 164

110. Link diagram for route map without voice, middle age group. .................... 165

111. Link diagram for route map without voice, older age group......................... 166

112. Link diagram for textual direction list, young age group. ............................ 167

113. Link diagram for textual direction list, middle age group........................... 168

114. Link diagram for textual direction list, older age group............................... 169

115 Link diagram for paper map, young age group ................................ 170

116. Link diagram for paper map, middle age group...................................... 171

117. Link diagram for paper map, older age group........................................ 172

118. Number if incidents for each safety-related error category shown compared across the three age groups

119. Number of inappropriate glances lasting over $2.5 \mathrm{~s}$ for each navigation condition compared across the three age groups..

120. Number of lane deviations for each navigation condition compared across the three age groups. ..........................................................................

121. Number of glances to the navigation display for drivers of differing area

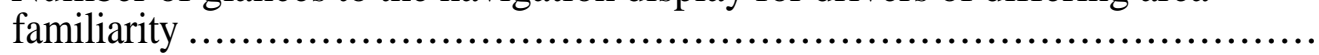

122. Number of glances to the roadway for drivers of differing area familiarity ...

123. Duration of glances to the navigation display for each navigation condition compared across drivers of differing area familiarity....

124. Number of glances to navigation display for each navigation condition compared across drivers of differing area familiarity.

125. Variance in lateral acceleration for drivers of differing area familiarity

126. Amount of time required to plan a trip shown for each navigation condition compared across drivers of differing area familiarity....

127. Number of navigation-related errors by type compared across drivers of differing area familiarity.

128. Number of navigation-related errors by type committed on local users' first drive, compared across each navigation condition....

129. Number of navigation-related errors for each navigation condition, compared

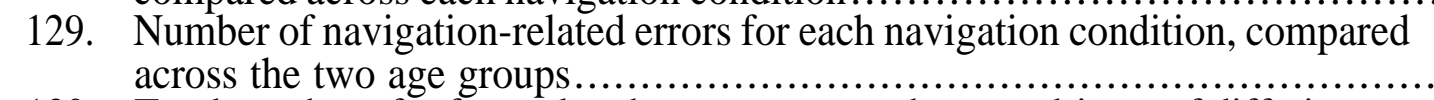

130. Total number of safety-related errors compared across drivers of differing area familiarity and age.

131. Number of lane deviations compared across drivers of differing area familiarity and age.

132. Number of lane deviations for each navigation condition compared across drivers of differing area familiarity and age. 
133. Eye glance link diagrams for the TravTek Camera Car Study and Etak Navigator Study paper map conditions....

134. Link diagrams for the Etak navigator and TravTek route map without voice conditions

135. Link diagram for the Etak memorized route condition.

136. Link diagrams for the TravTek paper direction control condition

137. Link diagrams for TravTek turn-by-turn conditions and route map with voice condition.

138. Questionnaire ratings of the usability of the TravTek route map from the Rental User Study....

139. Questionnaire ratings of the usability of the TravTek guidance display from the Rental User Study

140. Questionnaire comparison of the TravTek route guidance modes from the Rental User Study

141. Percentage of time renters spent using the TravTek visual configurations.

142. Percentage of time local users spent using the TravTek visual configurations

143. Percentage of time renters spent using the TravTek voice configurations.

144. Percentage of time local users spent using the TravTek voice configurations.

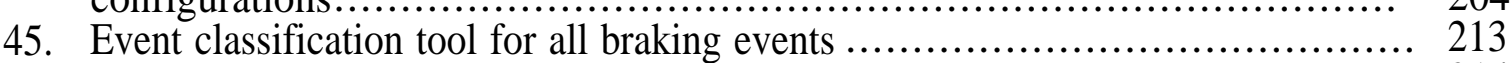

146. Event classification tool for all glances $>2.5$ events .............................. 214

147. Event classification tool for all inappropriate speed events ............................... 215

148. Event classification tool for all intersection error events .............................. 216

149. Event classification tool for all merging / lane change events ..................... 217

150. Event classification tool for all lane deviation events .................................. 218

151. Event classification tool for all cornering / turn events ............................. 219 
1. Accident involvement rates (per 100 drivers in each group). ............................. 9

2. Which navigation configuration(s) results in the least driving task intrusion? .......... 10

3. Which navigation configuration(s) results in the best navigation performance? ....... 13

4. Can any of the TravTek configuration designs be improved? ........................ 16

5. Do any of the navigation configurations result in unsafe behavior?................... 18

6. Trigger criteria used by the experimenter in the vehicle for further analysis. ............ 22

7. ANOVA's testing differences in glance length to the navigation aids and roadwayrelated features................................................................ 56

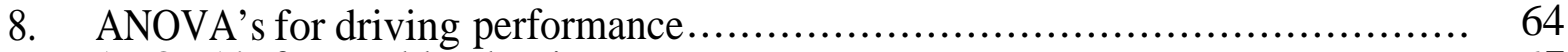

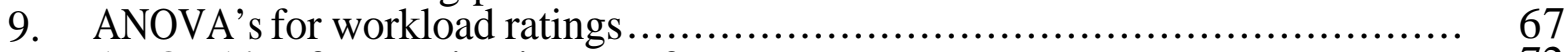

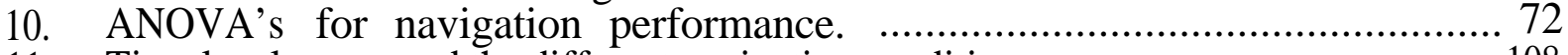

11. Time local users used the different navigation conditions ....................... 108

12. ANOVA's for driver performance measures addressing driver age ................. 110

13. ANOVA's for driver performance measures addressing navigation condition .......... 113

14. ANOVA's for navigation performance addressing age. …....................... 123

15. ANOVA's for navigation performance addressing navigation condition .............. 124

ANOVA's for performance indicative of ease of use addressing age .................... 129

17. ANOVA's for performance indicative of ease of use addressing navigation condition ........................................................................ 130

18. ANOVA's for driver performance measures addressing navigation condition and age. ....................................................................... 141

19. ANOVA's for navigation performance for age. ……................................ 149

20. ANOVA's for workload for display by age..................................... 153

21. ANOVA's for driving performance measures addressing driver type and age ....... 176

22. ANOVA's for driving performance measures addressing navigation condition

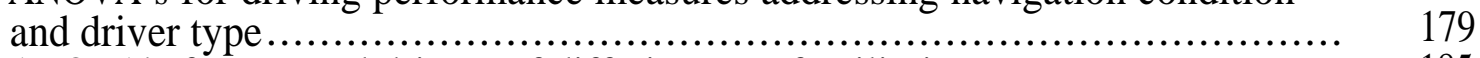

23. ANOVA's for age and drivers of differing area familiarity. ........................ 185

24. ANOVA's for navigation condition and drivers of differing area familiarity. ......... 186

25. Workload ANOVA's for area familiarity by age. ................................. 190

26. Workload ANOVA for navigation condition and drivers of differing area familiarity. ............................................................................... 191

27. Detailed error number description and assigned potential severity. ...................... 221 


\section{OVERVIEW}

The TravTek Camera Car Study was one of a comprehensive series of TravTek research evaluations. The purpose of the Camera Car Study was to provide a detailed evaluation of driver performance and behavior while operating the TravTek system. Although a number of navigation and information systems have been conceptualized or developed, few empirical evaluations of the safety and usability of various designs exist in the open literature. Of those research studies in existence, none have tested a device with the functionality of the TravTek system or other systems that will follow in the future. A primary means to collect the data required to assess the usability and safety of the TravTek system was an instrumented "camera car," which was developed to provide comprehensive driving performance and behavior measurement capability.

Many important usability and safety issues fell under the umbrella of the Camera Car Study. A camera car system and study allowed (1) more precise/detailed measurement and analysis of driving performance and behavior, and (2) reasonable control over many environmental factors (roadway types, subject demographics, time of day) necessary for interpretation of changes in driving performance and behavior.

A total of six navigation test configurations were used for the Camera Car Study. These included:

- TravTek route-map display.

- TravTek route-map display with supplementary voice guidance.

- TravTek symbolic guidance-map display.

- TravTek symbolic guidance-map display with supplementary voice guidance.

- Paper map.

- Paper textual direction list.

The first four conditions enabled researchers to assess the TravTek screen and/or voice configurations with respect to driving performance, system usability, and safety. The paper map and direction list conditions were included so that the TravTek system could be compared to the navigation baseline conditions. Any navigation technique will require greater attention demand than circumstances of non-navigation driving. Inclusion of the two most common navigation techniques as baselines provided an accurate methodology to measure TravTek costs and benefits.

The primary research issues associated with the Camera Car Study included the following:

- Which configuration(s) resulted in the least driving task intrusion?

- Which configuration(s) resulted in the best navigation performance?

- Could any of the TravTek configuration designs be improved?

- Did any of the navigation configurations result in unsafe driving behavior?

- Did driving performance, navigation performance, and driving safety vary as a function of TravTek experience?

- Did driving performance, navigation performance, and driving safety vary as a function of driver age?

- Did driving performance, navigation performance, and driving safety vary as a function of type of user (i.e., local or visitor) and area familiarity?

The issues of experience, age, and type of user were specifically included as part of the camera car test plan because previous research has shown that they strongly affect driving and/or navigation. 
A variety of driving and navigation performance measures were collected to assess differences between the six navigation configurations and the users groups of interest. In addition, a safety analysis assessing near misses and driver errors was conducted. The results of these analyses are discussed for each issue below.

\section{Which configuration(s) resulted in the least driving task intrusion?}

A subjective measure of the drivers' workload indicated that the paper map control condition and the TravTek route-map without voice condition were the most difficult to use. The highest visual attention demand (when considering eye glance and driving performance data ) was created by the route-map without voice condition. This was evident when considering both the number of glances to the TravTek display and the single display glance length, Relatively speaking, drivers were required to look longer at this display configuration to retrieve the required information. This demand was substantially reduced with the addition of voice guidance in conjunction with the route-map display.

In contrast, in terms of number of glances, the paper map condition required little visual attention even though the single display glance time was high. In contrast, the number of abrupt braking maneuvers, mean speed, and workload ratings indicated that the paper map intruded upon the primary task of driving. This condition required high "cognitive attention."

\section{Which configuration(s) resulted in the best navigation performance?}

The navigation performance results revealed that the TravTek and paper direction list conditions resulted in shorter overall trip times than the paper map control condition. In addition, drivers got lost the most while using the paper map. The three TravTek conditions which provided turn-by-turn information in at least one form had uniformly positive navigation performance results. Drivers were able to understand the required turnby-turn information (whether the information was provided by voice, visual display, or both), in order to efficiently and effectively get to their destination . The paper direction navigation condition required relatively short planning and driving times, as well as low workload levels. The paper directions simulated a computer-generated list found at some rental car counters; but featured a particularly large and legible font and distances to each turn. It was apparent that presentation of effective turn-by-turn information, by some means, significantly improved navigation performance. It was less critical whether this information was provided in a textual list (which could easily be provided on a CRT display), turn-by-turn graphic, and/or voice instruction. The situations where navigation performance was less effective was when next turn information was provided less effectively; the paper map (as indicated by both time and errors), and the route-map without voice (as indicated by the error data).

\section{Could any of the TravTek configuration designs be improved?}

The results of usability and ease of learning performance measures indicate that the TravTek navigation conditions were easier to use and learn than the paper map. The textual direction list was also as easy to learn and use as the TravTek navigation configurations. Both trip and planning times were significantly longer when using the paper map relative to the other navigation configurations. In addition, due to increased planning and driving times, significantly more time was spent stopped while using the paper map control condition when compared to other navigation conditions. This indicates that the overall ease of learning and usability of TravTek was relatively effective. Of the TravTek navigation configurations, the route map without voice appeared to be the most difficult to learn and use. This was due primarily to the difficulty drivers encountered identifying 
turns. The in-vehicle experimenter observed that novice drivers preferred the voice over the non-voice conditions. Some of the local users reported that once they learned the system, the voice was no longer needed for routine driving. However, if a driver programmed an unfamiliar route, the voice was typically turned back on. Younger local user drivers also reported that they frequently switched between the turn-by-turn route guidance and the route map. Local users reported that the route map gave good route planning awareness and the turn-by-turn display gave good immediate turn guidance.

\section{Did any of the navigation configurations result in unsafe driving behavior?}

No accidents and very few near misses resulted during the Camera Car Study. Therefore, the effects of the navigation configurations on driving safety were assessed by analyzing the near-miss events and safety-related errors. Errors were classified across different levels of potential severity, environmental proximity, and whether or not they were directly caused by use of a navigational device. Specific measures of performance were also taken for defined error types such as lane deviations, braking errors, etc. The results of the analysis show that there were considerably more safety-related errors in the TravTek routemap without voice condition than currently used methods of navigating (paper map or paper directions). The other TravTek configurations also had a substantially lower number of safety-related errors than the route-map without voice condition. Turn-by-turn with voice had a low and comparable number of safety-related error occurrences when compared to both a paper map and paper directions. The conditions of route-map with voice and turn-by-turn without voice had a similar number of errors and were slightly higher in many measures than the three lowest conditions. It was also determined that adding voice to a visual TravTek display generally improved overall safety performance.

\section{Did driving performance, navigation performance, and driving safety vary as a function of TravTek experience?}

Driving performance measures indicated that as drivers gained more system experience, they made fewer and shorter glances to the navigation system than novice drivers. Consequently, experienced drivers made more glances to the roadway environment. Experienced drivers were also able to plan a trip and drive to their destinations in less time than novice drivers. Analyzed safety aspects indicate that fewer unsafe incidents occurred as drivers gained experience. The larger number of safety-related incidents associated with the route-map without voice condition were substantially mitigated in an experienced group of local users.

\section{Did driving performance, navigation performance, and driving safety vary as a function of driver age?}

Driver age is an important factor to consider in the design of navigation systems. This study corroborates previous age research that showed significant effects on driver attention demand. Older drivers (65+) consistently showed decreased performance in navigating, increased navigation-related eye glance duration, increased workload, and longer planning and trip times. As the attention demand of navigation displays increased, older drivers reduced the amount of time scanning the left and right forward view. For older drivers this is an important consideration, since their useful visual field of view is reduced with age. Older drivers in particular had less difficulty in the TravTek conditions that provided turn by-turn guidance relative to the paper map control condition. 


\section{Did driving performance, navigation performance, and driving safety vary as a function of type of user (i.e., local or visitor) and area familiarity?}

Visitors drove more cautiously, but made more glances to the navigation aids when compared to local users. In addition, visitors went off-route and got lost less frequently than local users. Visitors apparently were more careful in driving and navigating to their destinations.

\section{Summary}

In summary, the TravTek route-map without voice had the greatest impact on the driving task and was the least safe of all the navigation conditions tested. However, the negative safety aspects of the display were substantially tempered by driver experience and the addition of a voice guidance supplement. In addition, other TravTek studies have shown that drivers used voice and the turn-by-turn guidance visual display most often while navigating when given a choice. Therefore, giving drivers a choice of navigation conditions will further mitigate this safety finding.

The TravTek conditions which provided turn-by-turn guidance either by visual display, voice or both, proved to be usable means of navigation, relative to the control conditions. Specifically in the case of the paper map, TravTek required about one-half as long to reach destinations designed to be $20 \mathrm{~mm}$ away. The route-map without voice was less usable than the other TravTek conditions or a paper direction list.

A primary finding of this research was that turn-by-turn guidance information (whether presented verbally, in a well designed textual list or by a graphic display), enhanced performance, usability, and/or safety compared to alternatives which provide holistic route information. For this study, the TravTek turn-by-turn with voice condition and a paper direction list, provided the best overall performance. The TravTek turn-by-turn without voice and route-map with voice conditions also provided good overall performance. 


\section{INTRODUCTION}

The purpose of the TravTek Camera Car Study was to provide detailed evaluations of driver performance and behavior while operating the TravTek system. In general, the goals of the Camera Car Study were to:

- Provide detailed evaluations of driver/system interactions including the effect of system use on the composite driving task.

- Provide evaluations of the navigation effectiveness including the usability of the predrive and drive functions of the TravTek system.

- Provide investigations into possible safety benefits and hazards associated with the use of the TravTek system.

To collect the data required to achieve the goals described above, an instrumented camera car was developed to have comprehensive driving performance and behavior measurement capability.

This section presents a brief background discussion, describing relevant issues associated with in-vehicle navigation and information use.

\section{BACKGROUND}

The TravTek Driver Interface was created and developed to provide navigation, services and attractions directory, and roadway incident and traffic information to the driver.(t) The primary design objectives for the design of this navigation system interface were to:

- Save the drivers time and money by providing more effective navigation information.

- Alleviate stress and increase driving enjoyment by providing easy access to value and convenient location information.

- Maintain and improve safe driving performance during system use and to do the same in emergency response by providing information for avoiding hazards and responding to emergencies.

- Alleviate traffic congestion by improving roadway efficiency.

This interface design was aided by several human factors evaluations made by the Etak Navigator, additional navigation systems, and preliminary research of the TravTek navigation system conceptual designs.

\section{Study Rationale}

A number of in-vehicle navigation and information systems have been marketed or are currently under development. Such systems vary greatly with respect to functionality and design. Although a number of navigation and information systems have been conceptualized or developed, very few safety and usability empirical evaluations exist in the open literature. Of those research studies in existence, none have tested a device with the functionality of the TravTek system or future systems. Therefore, there were many important usability and safety issues which fell under the umbrella of the Camera Car Study objectives. A Camera Car Study allowed (1) more precise/detailed measurement and analysis of driving performance and behavior, which was required to assess usability and safety of the system, and (2) reasonable control over many environmental factors (roadway 
types, subject demographics, time of day) necessary for interpretation of changes in driving performance and behavior.

\section{Relevant Literature and Past Research}

\section{Etak Navigator Studies}

The Etak Navigator was comprehensively evaluated in several human factors studies. $(2,3,4)$ These studies addressed the attention demands imposed by the Etak upon the driver, the effectiveness and efficiency of the navigation system, and driver adaptation behavior to the navigation system. Each of these studies was performed on the road using an instrumented camera car. These studies revealed that several of the Etak functions required a high degree of attention compared to other automotive tasks. Despite this fact, the Etak was found to be a usable and somewhat useful device that could potentially be improved by conceptual and design changes. On the basis of the Etak study results, the investigators recommended several modifications to the driver interface for future systems. These medifications included:

- Automated route selection.

- Automated zoom capability.

- Simplified information displays.

- Path feature for route planning.

Simplified information display and automated route selection have since been included as part of the TravTek system.

\section{Additional Navigation System Studies}

Several navigation systems have been developed in Japan and Europe. As an example, Autoguide has been publicly launched as part of a European project concerned with electronic systems (PROMETHEUS).(5,6) However, because many of these systems are still in their infancy, only limited usability and safety analyses have been performed. Therefore, this body of literature was of limited use in the development of the TravTek Camera Car Study.

A relatively recent research project, "Pathfinder," included traffic congestion information.(7) However, the usability of the system has not been a focus of the Pathfinder research plan. The technology used for Pathfinder does not provide information such as automatic route planning and replanning. Such information may prove to be critical from the standpoint of usability or convenience, if the system is to successfully encourage drivers to actually reroute a trip based on traffic congestion. Therefore, Pathfinder data will be of limited use in addressing TravTek Driver Interface issues.

\section{Preliminary TravTek Research}

Recommended modifications of the Etak Navigator and additional navigation system studies were included in a preliminary conceptual design of TravTek. This early conceptual design was subjected to several laboratory usability tests.@)

Testing the "predrive" functions revealed that manual route selection and browse map/area traffic scanning were difficult to use. It was the opinion of the investigators that since they were of marginal value to the overall system, these functions could be eliminated.

Additional testing also revealed that system labeling, nomenclature, and messaging should 
be tested thoroughly in order to minimize errors of confusion and/or required instruction. Furthermore, a larger control/display screen was recommended; however, in cases where a small screen was the only option, a system with a somewhat limited functionality was recommended. For the TravTek system, the TravTek design team included an automatic route selection feature for driver convenience.(r) In addition, the design team subjected nomenclature and labeling used in the system to comprehensive usability testing.

Evaluation of the "drive" functions during the TravTek design process included testing of the visual attention demand requirements. To reduce the attention demand, the inclusion of a turn-by-turn guidance map was strongly advised for future systems. Additional recommendations included:

- Directly providing critical information (e.g., next turn distance).

- Increasing the salience of critical roadway names through highlighting, the elimination of other street names and/or increasing the lettering size.

Each of the above recommendations was incorporated into the TravTek Driver Interface.(t)

\section{PURPOSE OF TEST}

The purpose of the Camera Car Study was to provide detailed data regarding interaction with four versions of the TravTek in-vehicle system. These versions included a moving map display showing a route from an origin to a destination, a turn-by-turn graphic showing the next turn information, and the moving-map and turn-by-turn configurations in conjunction with a voice guidance option. Each of these configurations is described in the next section.

The detailed data collected as part of the Camera Car Study were used to:

- Help identify the best of the four options and/or identify deficiencies in any part of the options.

- Provide data on what the TravTek in-vehicle system contribution is to safety; to include measures of increases or decreases in driver workload and out-of-thewindshield monitoring.

- Identify safety hazards associated with the in-vehicle systems.

- Describe changes in driver behavior as a function of experience in the use of the invehicle system, to:

- Support development of training procedures.

- Support design modifications to reduce training experience requirements.

- Support understanding of the impact of learning on safety and hazard factors.

- Support refinement of in-vehicle design.

To gain insight into the design, usability, and safety system aspects, a number of variables and measures were addressed. The Camera Car Study was conducted using a variety of subject demographic and roadway/traffic conditions. Demographic variables varied by age, gender, visitor versus local users, and novice versus practiced users. Roadway variables included roadway type and vehicle speed. Driving performance and behavioral data, as well as preferences and perceptions regarding specific system aspects were also measured.

\section{OBJECTIVES}

To achieve the objectives of the Camera Car Study, a number of issues were addressed. These issues included aspects of driver performance and safety, as well as differences in 
the subject population (e.g., age effects). Although a number of issues were of interest, they were not all mutually exclusive. For example, the safety evaluation performed as part of the Camera Car Study utilized measures collected to address several other issues (i.e., driving task interference, navigation performance, and configuration design for usability, as described below). Note that although the issues are discussed separately in this section, they are all addressed as part of a single experiment. That is, a single experiment included measures of performance that were utilized to assess each issue.

The primary research questions associated with the Camera Car Study included:

- Which configuration(s) resulted in the least driving task intrusion?

- Which configuration(s) resulted in the best navigation performance?

- Could any of the TravTek configuration designs be improved?

- Did any of the navigation configurations result in unsafe driving behavior?

Additional, related research questions include; did driving performance, navigation performance, and driving safety vary as a function of TravTek experience? Driver Age? Type of user (i.e., local or visitor) and area familiarity?

Experience, age, and type of user were specifically addressed as part of the camera car Test Plan, since previous research showed that they strongly affect driving and/or navigation. A previous study testing an in-vehicle navigation system showed that subjects spent significantly more time looking at a display (at the expense of looking out of the window) than they spent looking at a paper map.(3) The authors hypothesized that a novelty effect, characterized by drivers devoting spare visual time to the display (since it was new and different), was largely the cause of this finding. This hypothesis was supported by a general absence of driving performance degradation in the navigation system condition. Therefore, driver experience with a navigation system may bring about a drastic change in visual scanning behavior once the novelty of a system wears off.

Age has been shown to be an important factor in a number of driving-related studies. In a navigation system evaluation, Dingus, et al. found that driving and navigation performance for drivers over age 50 degraded compared to drivers ages 18 through 25 and 35 through 45.(4) Additionally, both younger and older drivers had higher accident rates in general than "middle-aged" drivers. This difference in accident rate (shown in table 1) emphasizes the need to include age as a Camera Car Study issue.

A number of researchers have shown that navigation behavior, including choosing one type of navigation information over another, changes with area familiarity.(9) In addition, the accident rate for rental car users is probably different than the overall accident rate. The causal factors for any difference would likely include both vehicle and area unfamiliarity, but the contribution of these factors to any effect is unknown. Therefore, since TravTeklike systems will most likely be used in the future by both commuters and rental car users, this issue requires study in order to assess the safety risks and benefits of such systems.

Additional factors have been shown to affect navigation or driving performance. Although, these additional factors generally have less of an effect than the factors described above, several of them required consideration to account for the variance introduced into the camera car data. These variables included: gender, map-reading ability, road type and traffic density. These were not treated as independent variables because of the complexity factors contributing to driving and navigation performance variance. In a field study such as the Camera Car Study, limitations had to be imposed on the number of factors, because of availability of resources and the experimental design feasibility. Therefore, neither the 
Table 1. Accident involvement rates (per 100 drivers in each group).

\begin{tabular}{|c|c|}
\hline Age Group & Accident Rates \\
\hline Under 20 & 36 \\
\hline $20-24$ & 37 \\
\hline $25-34$ & 25 \\
\hline $35-44$ & 18 \\
\hline $45-54$ & 14 \\
\hline $55-64$ & 13 \\
\hline $65-74$ & 14 \\
\hline $75+$ & 23 \\
\hline
\end{tabular}

secondary factors described above nor additional factors of potential interest such as fatigue, alcohol/drug use, day versus night, and weather conditions, were specifically addressed as part of this research.

The following sections that follow present the methodology developed for addressing each primary issue by describing:

- Camera car objectives with respect to each issue.

- Hypotheses associated with each objective.

- Measures of Effectiveness (MOE's) used to assess the attainment of the objectives.

- Measures of Performance (MOP's) that together constitute a MOE.

- Sources of MOP data.

- Methods used to analyze MOP's and MOE's.

The hypotheses tested support the specific objectives described for each issue. No specific hypotheses were advanced as to the specific outcome of any independent variables tested. The following discussion, MOE's are conceptual variables or constructs. MOP's are, in contrast, the directly measured variables. Therefore, each MOE was operationally defined in terms of one or more MOP's.

\section{ISSUE 1: WHICH NAVIGATION CONFIGURATION(S) RESULTED IN THE LEAST DRIVING TASK INTRUSION?}

Table 2 summarizes the navigation configurations in the Camera Car Study that resulted in the least driving task intrusion. The reason for addressing this issue was to provide a rank ordering (or grouping) of configurations based on driving performance measures, not to directly assess whether one or more navigation configurations were unsafe per se. 
Table 2. Which navigation configuration(s) results in the least driving task intrusion?

\begin{tabular}{|c|l|l|l|}
\hline \multicolumn{1}{|c|}{ OBJECTIVES } & HYPOTHESIS & $\begin{array}{c}\text { MEASURE OF } \\
\text { EFFECTIVENESS }\end{array}$ & $\begin{array}{c}\text { MEASURE OF } \\
\text { PERFORMANCE }\end{array}$ \\
\hline $\begin{array}{l}\text { Assess driving task } \\
\text { intrusion associated } \\
\text { with each configur- } \\
\text { ation }\end{array}$ & $\begin{array}{l}\text { Driving performance } \\
\text { will vary depending } \\
\text { on which configur- } \\
\text { ation is used }\end{array}$ & $\begin{array}{l}\text { Driving } \\
\text { performance }\end{array}$ & $\begin{array}{l}\text { \# of unplanned lane } \\
\text { deviations } \\
\text { Duration of } \\
\text { unplanned lane } \\
\text { deviations } \\
\text { Steering wheel } \\
\text { variance } \\
\text { of steering reversals } \\
\text { Mean velocity } \\
\text { Velocity variance } \\
\text { Abrupt lateral } \\
\text { maneuvers } \\
\text { Abrupt braking } \\
\text { maneuvers } \\
\text { Percent of time } \\
\text { scanning } \\
\text { roadway/traffic } \\
\text { Navigation aid glance } \\
\text { time/\# } \\
\text { Workload }\end{array}$ \\
\hline
\end{tabular}

\section{Objectives}

An important objective of the Camera Car Study was to provide a detailed assessment of the effects of using the TravTek configurations on driving performance. An important aspect of this objective was to assess the attention required by each navigation configuration. One reason that it was important to provide systems with limited attention demands is that the driving task (including maintaining position in the lane, scanning the environment, or attending to other traffic) sometimes requires almost all of the driver's mental resources. Each driving-related task consumes some of these limited resources and, at times, few resources are left over for the execution of the navigation tasks. In addition, there are individual factors (fatigue, arousal, and individual differences) that affect the amount of available resources.

Present and future navigation system designers will be faced with tradeoffs for selection of a navigation configuration. Driving performance is an important element in this tradeoff, both in terms of which configurations are potentially unsafe (issue \#4) and which configurations are the least intrusive on driving, while still providing necessary information in a timely manner.

It was not only important to compare different navigation configurations; it was important to compare all of them to available alternative methods (most notably a paper map and a textual list of directions). It was certain that any navigation alternative would intrude on the driving task to some extent.(s) With this knowledge, the key question then became, "Compared to other alternatives, to what extent do TravTek navigation alternatives affect driving?' Therefore, it was important to assess any difference between TravTek display alternatives and currently used means of navigation. 


\section{Hypotheses}

It was hypothesized that driving task intrusion would vary as a function of the six configurations (four TravTek and two non-TravTek alternatives) used. Specifically, it was hypothesized that one or more of the MOP's described below would vary as a function of navigation technique.

\section{Measures of Effectiveness}

The MOE for this issue was driving performance. Driving performance in this context refers to the primary task of driving (i.e., maintaining position in the lane, scanning the environment, attending to other traffic, maintaining vehicle speed, assimilating environmental information, and executing required and desired maneuvers). Driving performance was operationally defined as a multivariate set of measures which individually addressed one or more aspects of the driving task.

\section{Measures of Performance}

The MOP's collected to assess driver performance for this issue were the following:

- Number of unplanned lane deviations.

- Duration of unplanned lane deviations.

- Steering wheel position variance.

- Number of steering wheel reversals greater than 6 degrees.*

- Average vehicle velocity.*

- Velocity variance.

- Number of abrupt lateral maneuvers.

- Number of abrupt braking maneuvers.

- Variance in lateral acceleration.*

- Mean negative longitudinal acceleration. *

- Variance in longitudinal and negative longitudinal acceleration.*

- Number and length of brake applications.*

- Percentage of time spent scanning the roadway environment

- Number and length of glances to the navigation aids.*

- Subjective measures of driver workload.

* Indicates additional measures that were not originally proposed in the camera car Test Plan.

The measures marked with an asterisk were added to the list of performance measures because they provided some unique insight into driver performance and could easily be derived. All of the above MOP's are described in detail in the following sections.

\section{N umber and D uration of Lane D eviations}

A lane deviation was defined as any part of the vehicle exceeding a lane boundary. Unplanned lane deviations provided a valuable face-valid measure of driving task interference resulting in performance degradation. Both the numbers and durations of deviations were measured. Increases in either measure indicated a degradation in driving performance. 


\section{Steering Wheel Position Variance and N umber of Large Steering Reversals}

Research has shown that changes in driver steering behavior occurred when driver attention changed.(10) In normal, low attention circumstances, drivers made continuous, smaller steering corrections to make up for roadway variance and driving conditions. These corrections were typically within the range of 2 to 6 degrees. As attention or workload demands increased, the frequency of steering corrections tended to decrease. Since the small centering corrections decreased, the vehicle tended to drift farther from the lane center, and a larger steering input was required to correct the position. These larger steering inputs generally exceeded 6 degrees and were referred to as large steering reversals. Since small corrections decreased and large corrections increased, an increase in the steering wheel position variance indicated high attention or workload requirements and a reduction in driving performance.

\section{Average Vehicle Velocity and Velocity Variance}

Vehicle speed, like lane position, can be considered a vehicle state which, at some level, had to be held constant in most circumstances. Therefore, for the same reasons described above for steering reversals, variations in velocity were used to evaluate performance. Drivers were required to make continuous adjustments in pedal displacement to maintain the correct speed. When driver attention was drawn away from the driving task, there was a tendency to maintain the foot in the same position. When drivers realized they were going (generally) too slow, the accelerator was depressed to a greater degree than was normal for a continuous adjustment. Research found velocity maintenance to be a sensitive measure to changes in the amount of attention demanded by secondary driving tasks.(11)

In addition to the research described above, average vehicle speed is also a valid measure of task demand. Previous research has shown that drivers adapt to increased task demand by modifying their behavior and driving more "cautiously."(12) One way that this modification was exhibited was in a decrease in vehicle velocity with increasing task demand.

\section{Lateral Acceleration Measures}

Abrupt lateral maneuvers, like large steering reversals, are indicative of a vehicle that is off lane center track due to driver inattention. Lateral acceleration measures were highly correlated to driver steering input. However, large lateral accelerations provide insight, in terms of magnitude, to the degree to which the vehicle is off-track. Therefore, lateral accelerations were used in large part to highlight large magnitude corrections.

\section{Longitudinal Acceleration M easures and Braking Data}

Braking behavior can also provide a sensitive measure of performance.(11) If drivers are looking away from the driving scene and glance back only to realize that an unanticipated event is occurring, the brake pedal must be depressed harder and the resulting deceleration is greater than in a normal attention situation. Longitudinal deceleration and brake pedal activation data were measured as part of the Camera Car Study.

\section{Time Spent Scanning the Roadway Environment and Navigation Aids}

The driving task requires constant scanning of the forward roadway, to the left and right of the forward roadway, and to the rear (via mirrors) to drive effectively and defensively. Therefore, a reduction in the time spent scanning these locations can be construed as a decrease in driving performance. However, as Antin, et al. found in evaluating the Etak 
Navigator, caution must be used when assessing eye glances.(3) Antin and his associates discovered an apparent novelty effect when comparing eye glance data from the navigator to that of a paper map. That is, subjects allocated most of their spare visual capacity to the Etak system presumably because they were novice users and it was a novel system. This resulted in a large percentage of time being spent looking at the navigator without a degradation in other driving performance measures.

It was anticipated that the novelty between the two TravTek visual display configurations would be approximately equal. Therefore, the percentage of time spent scanning the roadway and navigation aids was a useful measure for comparing the four display/voice configurations. The comparisons for the paper map and textual direction list conditions required more caution, since neither of these conditions were novel.

One of the reasons for the navigation experience issue (described in a later section) was to determine whether or not novelty effects subsided with regular system use. Therefore, visual scanning behavior was a primary measure for comparing novice and experienced users.

\section{ISSUE 2: WHICH NAVIGATION CONFIGURATION(S) RESULTED IN THE BEST NAVIGATION PERFORMANCE?}

Table 3 summarizes the navigation configuration that provided the best navigation performance.

Table 3. Which navigation configuration(s) results in the best navigation performance?

\begin{tabular}{|l|l|l|l|}
\hline \multicolumn{1}{|c|}{ OBJECTIVES } & HYPOTHESIS & $\begin{array}{c}\text { MEASURE OF } \\
\text { EFFECTIVENESS }\end{array}$ & $\begin{array}{c}\text { MEASURE OF } \\
\text { PERFORMANCE }\end{array}$ \\
\hline $\begin{array}{l}\text { Assess navigation } \\
\text { performance } \\
\text { associated with } \\
\text { each configur- } \\
\text { ation }\end{array}$ & $\begin{array}{l}\text { Navigation } \\
\text { performance will } \\
\text { vary depending on } \\
\text { which configuration } \\
\text { is used }\end{array}$ & $\begin{array}{l}\text { Navigation } \\
\text { performance }\end{array}$ & $\begin{array}{l}\text { Trip planning time } \\
\text { of wrong turns } \\
\text { Route replanning time } \\
\text { Time at zero speed } \\
\text { \# of stops } \\
\text { Reach destination } \\
\text { Travel time } \\
\text { Travel distance }\end{array}$ \\
\hline
\end{tabular}

\section{Objectives}

A primary objective of the TravTek Camera Car Study was to evaluate the navigation effectiveness, including the usability of the predrive (to a limited degree) and drive functions of the TravTek system. The camera car environment provided a unique opportunity to assess in detail not only differences in navigation performance, but the prospective causes of performance differences. Any navigation performance differences were evaluated with respect to precise timing and event occurrence by analyzing the record from the cameras and computer data contained in the car. Therefore, the Camera Car Study provided a more detailed record of causal factors of navigation performance differences than other TravTek studies (e.g., Study C2 - Orlando Test Network Study). 


\section{Hypotheses}

It was hypothesized that driver navigation performance would vary as a function of navigation configuration.

TravTek was designed to reduce the waste caused by navigation errors and getting lost. In addition, if TravTek planned better routes and drivers took those routes, shorter trip distance and shorter trip completion times should have resulted.

Although all TravTek conditions have equal advantage in trip planning, the various display conditions may vary in how well they communicate to drivers. Shorter, quicker trips should result from configurations that lead to fewer driver errors, and thus to fewer inadvertent diversions. Also, more easily interpretable visual and aural displays may reduce the slowing response that is common to drivers when they must attend to complex navigation information. Note that in the Camera Car Study paper, direction list control condition trip planning was, in effect, performed for the subject. That is, the subject was handed a list of directions at the beginning of a trip, instead of having to input a destination or peruse a paper map for an appropriate route. Even in the case where such a list would be computer generated, a destination would have to be input and a route calculated. Therefore, comparisons of trip planning times between the TravTek conditions and the paper direction list are not appropriate. Such cases are noted in the results section of this document.

Navigation errors were defined as deviations from a planned route. It was hypothesized that some conditions would lead to fewer such errors. If there were fewer errors, there should also be fewer stops for replanning or assistance, thus reducing travel time.

\section{Measures of Effectiveness}

The MOE for this issue was navigation perfomrance. Navigation performance, in this context, referred to the secondary task of navigating to a destination. Navigation performance was therefore defined as a multivariate set of measures which individually addressed one or more aspects of the navigation task.

\section{Measures of Performance}

\section{Navigation Performance}

The navigation performance MOE's were evaluated using the following measures:

- Trip planning time.

- Number of wrong turns.

- Number of missed turns.

- Time at zero speed.

- Number of stops.

- Reach destination (yes or no).

- Number of times the driver was lost.

- Travel time.

In five of the six camera car test conditions, a wrong turn was defined as deviation from TravTek or the paper direction list's planned route. However, once off the planned route, turns were not counted. Going straight through an intersection where a turn was planned also counted as a wrong turn. In the paper map condition, identification of a wrong turn was sometimes problematic since drivers varied their routes often. In the paper map 
condition, participants verbalized their plan and wrote down a sequence of planned turns before beginning. These turns were used to detect deviations from the plan. By encouraging participants to verbalize what they were doing, it was hoped that useful error data could be obtained for the paper map condition. In all conditions wrong turns were recorded by the experimenter utilizing an "event" button available on an experimenter control panel. In addition, off-route messages to the driver were recorded by the camera recording a view of the TravTek display.

When stops were made to obtain navigation information (e.g., route replanning or looking for signs), or for other reasons, time at zero speed and number of stops were calculated. Start and stop times for stops were recorded by the data collection computer. Drivers were encouraged to explain the reason for stops not related to road conditions. These explanations aided in attributing stops to navigation or other causes (e.g., mechanical difficulty). All driver commentary was recorded by a live audio channel that operated for all data runs. In addition the circumstances surrounding navigation-related stops were recorded by the in-vehicle experimenter.

One measure of performance was whether the subject completed the trip. Presumably, conditions that yielded significantly better trip completion rates were more usable. All subjects completed all navigation conditions within the $60 \mathrm{~min}$ per origin/destination (O/D) criteria. However, in some cases, subjects were "lost." Lost was operationally defined as being off-route for at least several minutes and not knowing how to correct the action without a stop and replan.

Trip planning time began when the observer handed the driver a card with a destination written on it, and ended when the driver placed the car in DRIVE. With the TravTek vehicle at an origin and in PARK, the subject was handed an index card containing an origin (present position) and destination identification. Before handing the card to the subject, the experimenter configured the system to display the main menu. Subjects entered the trip into the TravTek computer as instructed during training. Instructions constrained all subjects to the same route (FASTEST). Travel times were calculated from the point of placing the vehicle in DRIVE and ceased when the destination was reached and the car placed in PARK. To achieve the overall trip time, trip planning and travel times were calculated from the camera car data log systems via the experimenters control panel.

A primary purpose of the Camera Car Study was to assess the safety-related behavior of the driver when using the different TravTek configurations (as addressed in detail in the unsafe driving behavior section - issue 4). One aspect of this assessment was the identification of conditions leading to poor or unsafe navigation-related driving behavior. Therefore, assessment of navigation performance included the identification of data that could be used as indicators of questionable driver actions. The methodology that was used to identify and analyze such data is discussed in the section on unsafe driving behavior (issue 4).

\section{D river Perception of Configuration Contribution to Navigation Performance}

Driver perceptions of the usefulness of the various navigation configurations were collected via questionnaires. The questionnaires utilized the same questions addressed as part of the Orlando Test Network Study (OTNS). Since the number of camera car users was relatively small, these data were combined with the larger OTNS data to assess overall user impressions. 


\section{ISSUE 3: COULD ANY OF THE TRAVTEK CONFIGURATION DESIGNS BE IMPROVED?}

\section{Objectives}

Table 4 summarizes the approach for determining whether user interface design aspects could be improved for any of the TravTek configuration alternatives.

Table 4. Can any of the TravTek configuration designs be improved?

\begin{tabular}{|c|l|l|l|}
\hline OBJECTIVES & \multicolumn{1}{|c|}{ HYPOTHESIS } & \multicolumn{1}{c|}{$\begin{array}{c}\text { MEASURE OF } \\
\text { EFFECTIVENESS }\end{array}$} & $\begin{array}{c}\text { MEASURE OF } \\
\text { PERFORMANCE }\end{array}$ \\
\hline $\begin{array}{l}\text { Assess TravTek } \\
\text { imprerface design }\end{array}$ & $\begin{array}{l}\text { Learning ease will } \\
\text { differ with regard to } \\
\text { navigation } \\
\text { configuration } \\
\text { Ease of use will } \\
\text { differ with regard to } \\
\text { navigation } \\
\text { configuration }\end{array}$ & Ease of learning & $\begin{array}{l}\text { Trip planning time } \\
\text { Experimenter } \\
\text { observation } \\
\text { and length of } \\
\text { navigation glances } \\
\text { Maneuver } \\
\text { timing/abruptness } \\
\text { Trip planning time } \\
\text { Experimenter } \\
\text { observation } \\
\text { and length of } \\
\text { navigation glances } \\
\text { of required system } \\
\text { interactions } \\
\text { Maneuver } \\
\text { timing/abruptness }\end{array}$ \\
\hline
\end{tabular}

A primary objective for the TravTek Study was to evaluate user interface designs for each of the four TravTek configurations. This evaluation included two factors, the first was the usability of the configuration. Even given that the most efficient route was selected, if the configuration was unusable, the errors could negate any efficiency gain. The second factor was ease of learning of the configuration used. Many navigation systems, such as those in rental car fleets are utilized by novice users. Therefore, to allow optimal performance by novice users, it was important that the system be easy to learn. Ease of learning was primarily assessed by in-vehicle experimenter observation during the training period. For the Camera Car Study, the majority of subjects performed the required pre-drive functions with few errors using information gained for the training described in the method section. In the "drive" portion of the experiment, it was difficult to distinguish between errors associated with "ease of learning" or "ease of use" since each subject used each configuration only once in the visitor case, and twice (but 6 weeks apart) in the local user case. Therefore, the results of this analysis are primarily anecdotal observation,

Evaluations of learnability and usability must be conducted in context. That is, the learnability and usability of the TravTek system must be evaluated relative to currently existing methods of navigation (e.g., paper maps and textual direction lists). Researchers have found that memorizing a route from either maps or lists was difficult and not done well. In addition, remembering spatial map configurations or mentally reorienting a map is difficult for people and conflicts with the spatial task of driving.(13) Finally, some navigation tasks 
are difficult because the information is not always available or is it obscured (i.e., street signs). Therefore, drivers generally appreciate assistance in navigation.

\section{Hypotheses}

It was hypothesized that the ease with which drivers learn and use the system varied for each of the six configurations used (four TravTek and two paper map/direction list).

\section{Measures of Effectiveness}

This MOE encompassed the areas of ease of learning and usability. How easily would drivers learn and use the navigation system? While doing so, how was their navigation performance altered?

\section{Measures of Performance}

The MOP's collected to assess both learning and ease of use for this issue included trip planning time and number of wrong turns, as discussed for navigation performance. In usability evaluations, it was common to assess both a drivers task completion time and number of errors while using a particular system. In addition to these MOP's, the MOP's listed below will also be utilized to assess the MOE's for this issue.

- Maneuver timing/abruptness.

- Number and duration of glances to navigation system.

- Experimenter observation.

\section{Maneuver Timing/Abruptness}

If abrupt maneuvers are needed to stay on route, this may indicate a lack of timely or useful information. For each configuration, such circumstances were recorded via an onboard accelerometer that measured both longitudinal and lateral acceleration. The data stream from these two measures was analyzed post-hoc to identify accelerations that were greater than three standard deviations from the mean. In addition, an abrupt maneuver was recorded as an event by the experimenter via the experimenter control panel. A criterion for an "abrupt" maneuver was when the driver came close to missing a turn and had to brake firmly and/or was observed to be confused at a displayed turn.

\section{Number and Duration of Glances to the Navigation System}

The required number of glances and their durations are indicators of the level or user understanding of the systems information and usability. These data were recorded with the eye-glance camera and reduced in the laboratory.

Long (>2.5 s) glances to a configuration were assessed in the laboratory for possible usability or learnability concerns.

\section{Experimenter 0 bservation}

For each configuration, ease/difficulty of learning and use questions were assessed by the in-vehicle experimenter. A single observer, trained in human factors engineering with experience in usability testing, performed all of the data collection activities. 


\section{ISSUE 4: DID ANY OF THE NAVIGATION CONFIGURATIONS RESULT IN UNSAFE DRIVING BEHAVIOR?}

Table 5 summarizes the camera car approach to determining which (if any) navigation configuration resulted in unsafe driving behavior. The purpose of this issue was to determine, with the greatest degree of accuracy given the measurement state of the art, whether any of the navigation configurations tested resulted in unsafe circumstances.

Table 5. Do any of the navigation configurations result in unsafe behavior?

\begin{tabular}{|c|c|c|c|}
\hline OBJECTIVES & HYPOTHESIS & $\begin{array}{c}\text { MEASURE OF } \\
\text { EFFECTIVENESS }\end{array}$ & $\begin{array}{c}\text { MEASURE OF } \\
\text { PERFORMANCE }\end{array}$ \\
\hline $\begin{array}{l}\text { Determine if any } \\
\text { of the navigation } \\
\text { configurations } \\
\text { result in unsafe } \\
\text { driving behavior. }\end{array}$ & $\begin{array}{l}\text { Navigation } \\
\text { configurations may } \\
\text { cause unsafe driver } \\
\text { behavior }\end{array}$ & $\begin{array}{l}\text { Accidents } \\
\text { Near misses } \\
\text { Unsafe acts }\end{array}$ & $\begin{array}{l}\text { \# of accidents } \\
\text { Assessment of } \\
\text { accident causal } \\
\text { factors } \\
\text { Single eye glances > } \\
2.5 \mathrm{~S} \\
\text { Abrupt lateral } \\
\text { maneuvers } \\
\text { Abrupt braking } \\
\text { maneuvers } \\
\text { Unplanned lane } \\
\text { deviations } \\
\text { Dangerously close } \\
\text { headways } \\
\text { Turn tracking errors } \\
\text { Unsafe intersection } \\
\text { behavior } \\
\text { Late/inappropriate } \\
\text { reaction to an } \\
\text { external event } \\
\text { Unplanned speed } \\
\text { variation }>16 \mathrm{~km} / \mathrm{h} \\
\text { (10mi/h) } \\
\text { Stopping in unsafe } \\
\text { circumstances } \\
\text { Subjective workload } \\
\text { ratings for overload }\end{array}$ \\
\hline
\end{tabular}

\section{Objectives}

An important objective of the Camera Car Study was to assess, in detail, whether using any of the TravTek configurations affected driving safety. Drivers should have been able to use any navigation configuration or perform any required in-vehicle task without jeopardizing driving safety. In addition, during critical driving situations or emergencies, drivers should have been able to redirect and focus their attention to the driving task. As long as the demands imposed by a configuration leave sufficient resources for driving in all situations, driving safety should not have been reduced. 
Furthermore, it was expected that the TravTek system would enable the driver to avoid getting lost and to find gas stations and emergency services when required. In this manner, TravTek could provide a safety benefit, enhancing safety and security.

In order to determine if navigation system safety benefits outweigh risks, all instances of foreseeable use should be considered. Designers cannot rely on drivers to use the system safely and only in the manner intended.(14) Therefore, each available navigation configuration was evaluated to determine if it could be safely attended to or manipulated while driving.

Since some driving tasks require more attention than others, a broad cross section of driving safety circumstances must be evaluated.(15) In the context of navigation systems, circumstances include vicinity-of-a-turn situations (close enough to a turn or decision point that maneuver preparation was necessary), mid-route situations (a greater distance away from a turn or decision point), and anomalous situations (including presentation of offroute, congestion, and action information by the system).(16) Other driving circumstances that require consideration include two-lane streets, which require more attention than Interstates; curved roads, which require more attention than straight roads; and heavy traffic which requires more attention than light traffic. It would then follow that composite driving task attention and workload must be measured to ensure that drivers were allocating resources appropriately in circumstances with high resource demands (i.e., that the driver was effectively ignoring navigation in some situations).

As discussed in the driving task intrusion section (issue 1), any navigation alternative will intrude on the driving task to some extent. @) However, the key safety objective was to determine whether any alternative configuration results in unsafe driving performance or behavior.

The task of navigating an automobile is difficult for most drivers relative to driving to a known destination, is often not done well, and requires a significant investment of information processing resources.(17) It is much more difficult, in terms of intrusion on the driving task, to navigate to an unknown destination than it is to commute and monitor traffic in a familiar area. Therefore, the priority for the Camera Car Study was to evaluate the safety of drivers navigating to unknown destinations rather than of commuters driving on familiar routes.

\section{Hypotheses}

It was hypothesized that driving performance would degrade for one or more of the six navigation configurations (four TravTek, paper map, and direction list). Specifically, it was hypothesized that one or more of the MOP's described in unsafe driving behavior section (issue 4) would indicate unsafe driving behavior as a function of navigation technique and driving circumstance.

\section{Measures of Effectiveness}

There are two MOE's related to the issue of safety; accidents and near misses. Accidents that can be attributed to use of a navigation configuration provide hard evidence of a potential safety risk. Such accidents, however, must be taken in context with respect to exposure. That is, there is a risk of accidents at some probability level regardless of whether or not a driver is using a TravTek configuration to navigate to a destination. The key issue then becomes "Is there a greater risk of an accident while navigating with a TravTek configuration than with an alternative navigation configuration?' As discussed 
previously, navigation is a difficult task to perform while driving. Navigation requires searching the environment for cues and landmarks that are often hard to identify, memorizing or referring to maps or direction lists, and maintaining spatial orientation, all of which can interfere with the driving task. In attempting to proactively evaluate safety, it is often the case that accident data are of limited use.

Fortunately, estimates of the accident risk associated with a system can be established through the analysis of near-miss data. Near-miss is defined as "any navigation-related act which, in and of itself, creates the potential for a collision."

Near misses occur more often than accidents of any severity, as illustrated in figure 1 . This figure, known as Heinrich's Triangle, is used in other safety applications to get an estimate of the future accident rate by counting near misses. By counting the number of near misses associated with a given configuration and driving circumstance, it is sometimes possible to estimate the number and severity of accidents that would occur for given levels of market penetration. Unfortunately, the numerical tie between accidents and near misses does not yet exist for driving. The concept is useful for comparison of navigation conditions, since (1) no accidents occurred while driving the camera car, and (2) differences in numbers of near misses will reflect ordinal differences in accident rates atsome level.

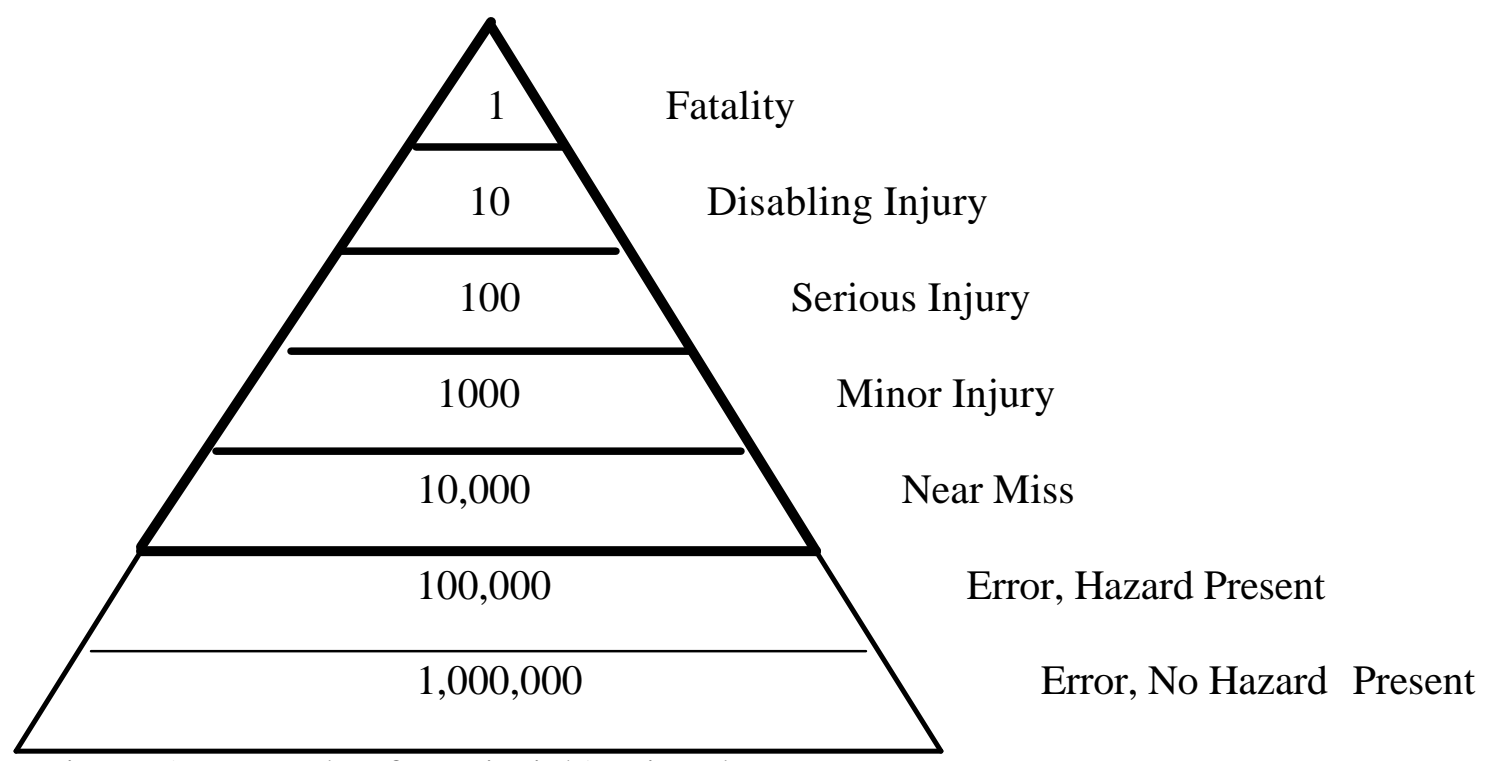

Figure 1. Example of a Heinrich's triangle

Also shown in figure 1 are two levels below near miss not commonly assessed as part ofthis method. These levels, dubbed "error, hazard present" and "error, no hazard present" refer to driving errors which do not constitute a true "close call." These categories were analyzed along with true near missesto provide additional safety-related data forcomparison. The relative magnitudes shown in figure 1 are hypothetical. However, regardless of their relative frequency, these measures provide a valuable means for direct comparison between the navigation conditions tested.

The classification of an event as a near miss was systematically evaluated using a prioririteria. These criteria were used to establish the severity of an unsafe act based on thepotential accident consequences. The criteria used included type of accident and associatedinjury potential, vehicle speed, presence or absence of proximal traffic, and roadway typeincluding the presence of proximal obstacles. 
Potential accident consequences were established by two independent raters who used the above criteria. The classification scheme was based on the most severe, reasonably probable potential outcome, including fatality, disabling injury, serious injury, minor injury, and no injury. A detailed description of this evaluation process can be found in the results section for issue 4 .

\section{Measures of Performance}

The MOP's collected to assess safety included behaviors that indicated the presence of an unsafe act. These MOP's actually served as criteria for further assessment of the presence and severity of an unsafe act in laboratory analyses. These "trigger" criteria, for which additional analyses were performed, included the following:

- Single display glances greater than $2.5 \mathrm{~S}$.

- Abrupt lateral accelerations.

- Abrupt braking behaviors.

- Unplanned lane deviations.

- Late/inappropriate reaction to an external event (including: inadvertent failure to observe related safety signs and signals, and closeness of approach to other vehicles, e.g., such as actions that cause another driver to take evasive action).

- Unplanned speed variations greater than $16 \mathrm{~km} / \mathrm{h}(10 \mathrm{mi} / \mathrm{h})$.

- Stopping in unsafe circumstances.

- Unsafe intersection behavior.

- Turning track and other turn errors.

- Dangerously close headways.

- Subjective workload ratings indicating overload.

Any point in the data stream that had a trigger event mark associated with it was subjected to detailed analysis. This analysis included detailed review of the videotape record generated by the camera car. A detailed description of this procedure is described in the results section for issue 4 .

The activation of the trigger criterion was automatic for cases in which where the data were flagged by the computer, and manual for cases that required the judgment of the in-vehicle experimenter. A detailed set of descriptions of all circumstances that constitute trigger events used by the experimenter is given in table 6 .

\section{Single Display Glance Time}

Glance duration was recorded such that data reduction in the laboratory resulted in the duration of each driver glance to the nearest $0.1 \mathrm{~S}$. Of particular relevance to the safety of utilizing the system were the durations of single glances to the display (or map) configuration in question. Bhise, Forbes, and Farber have stated, based on speed and travel distances, that any single display glance greater that $2.5 \mathrm{~S}$ in a moving vehicle is inherently dangerous.(18) Based on this research, this value was used as a criterion to assess instances of unsafe behavior. Note that although this criterion value has been described as "inherently dangerous" it is not known how long glances away from the roadway correlate with accidents. It is clear, however, that in many circumstances, such glances are inappropriate and increase the potential for crashes. 
Table 6. Trigger criteria marked by the experimenter in the vehicle for further analysis.

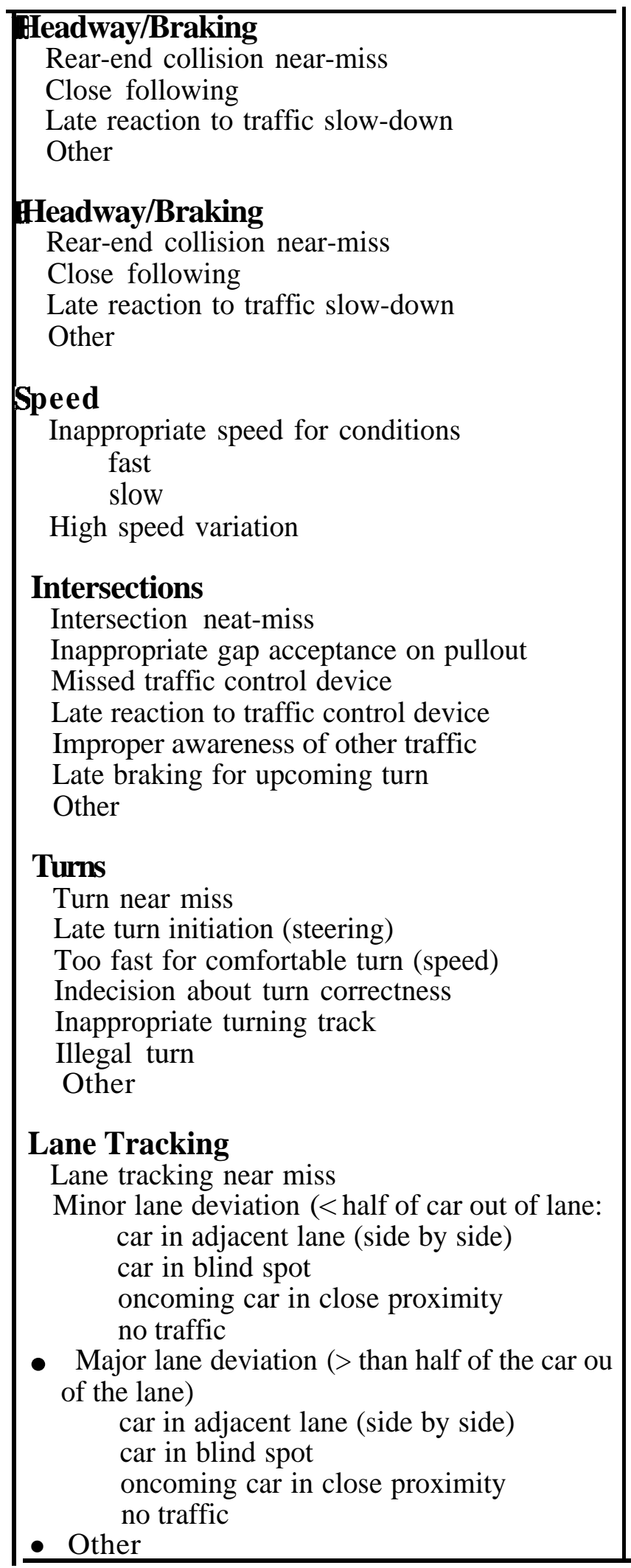


Table 6. Trigger criteria marked by the experimenter in the vehicle for further analysis (continued).

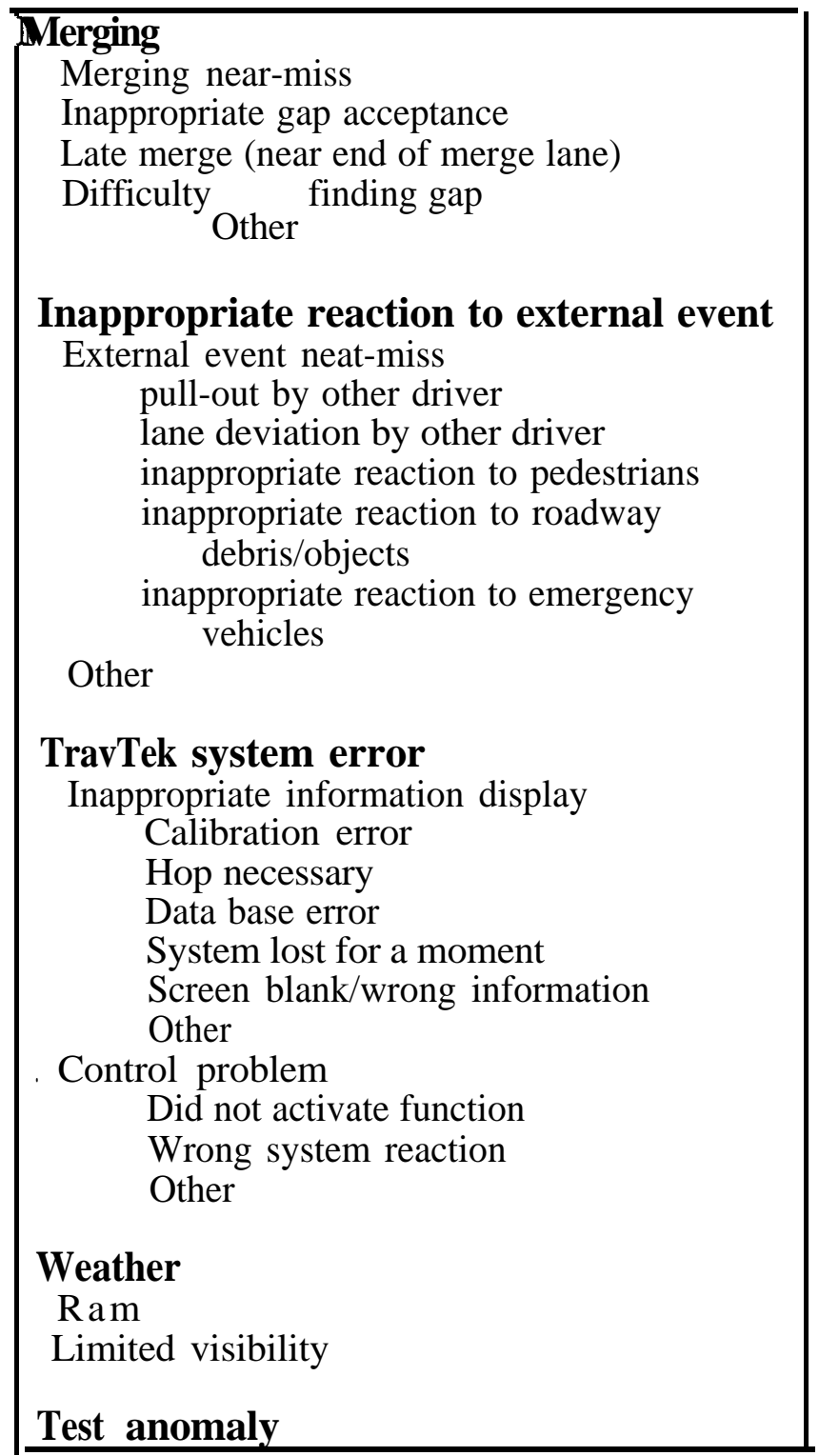

\section{Abrupt Lateral and Braking Maneuvers}

Lateral and longitudinal accelerations were automatically recorded by camera car sensors. Abrupt lateral and longitudinal accelerations could be indicative of driver inattention and therefore an unsafe act. Accelerations above $3.9 \mathrm{~m} / \mathrm{s}^{2}(0.4 \mathrm{~g})$ served as a trigger for event analysis.

\section{Unplanned Lane D eviations and Turning Track Errors}

An unplanned lane deviation is a face valid indicator of driver inattention and collision potential. In the laboratory, lane deviations were classified and timed from the lane-track camera record. A turning track error is similar to a lane deviation except that they occur in 
turns where no lane markers are typically present. Each unplanned deviation served as a trigger event for further analysis.

\section{Late/Inappropriate Reaction to an External Event}

Because an inappropriate reaction to an external event in and of itself creates the potential for a collision, it was used as a trigger event. The in-vehicle experimenter provided a record of inappropriate reactions via the "event" button available on the experimenter control panel. To minimize the probability of missing important safety data, the experimenter used the event key somewhat liberally. All trigger events were reviewed on videotape to establish the cause.

\section{Unplanned Speed Variation in Excess Of $16 \mathrm{~km} / \mathrm{h}(10 \mathrm{mi} / \mathrm{h})$}

Unplanned speed variation indicates driver inattention to the driving task. The criterion of $16 \mathrm{~km} / \mathrm{h}(10 \mathrm{mi} / \mathrm{h})$ was selected as a trigger value for two reasons; a variation of $16 \mathrm{~km} / \mathrm{h}$ $(10 \mathrm{mi} / \mathrm{h})$ indicates driver inattention for a relatively long period of time, and such a variation means that the closing rate between the camera car and proximal traffic was relatively high (increasing the accident potential). This criterion level was pretested to determine if, in fact, it appeared reasonable in the field. Speed variations were automatically determined by the in-vehicle computer.

\section{Stopping or Slowing in Unsafe Circumstances}

If a subject stopped or slowed to au unsafe speed in a location that created the potential for a collision, the in-vehicle experimenter treated this as a trigger event. "Unsafe" was operationally defined as any circumstance where slowing, stopping, or accelerating created the potential for a collision. The in-vehicle experimenter assessed whether stopping or slow driving circumstances were unsafe and recorded the occurrences via the "event" key on the experimenter control panel.

\section{Unsafe Intersection Behavior}

Any circumstance where unsafe behavior occurred in the vicinity of an intersection was flagged by the in-vehicle experimenter on the videotape. Such circumstances included improper visual scanning, failure to appropriately yield right-of-way, and improper reaction to a traffic control device.

\section{Dangerously Close Headways}

Utilizing the videotape record from the forward-view camera, a laboratory assessment of the appropriateness of vehicle headway (given driver visual scanning behavior) was performed. Those instances where headway was too close to allow the driver to react appropriately to an unexpected event were treated as triggers and subjected to further analysis.

\section{Mental Workload}

During each camera car data collection run, a subjective workload measure was collected on a periodic basis. The subjective scale used required the subject to rate three dimensions (time stress, psychological stress, and mental effort) as low, medium or high. (See the TravTek Yoked Driver Study - Cl for a complete description of the subjective scale used). Any workload response that indicated subject overload was treated as a trigger event and subjected to further laboratory analysis. 


\section{ISSUE 5: DID DRIVING PERFORMANCE, NAVIGATION PERFORMANCE, AND DRIVING SAFETY VARY AS A FUNCTION OF TRAVTEK EXPERIENCE?}

Based on prior research, it is apparent that driver navigation performance and behavior vary as a function of navigation system experience.@) Therefore, as described in the research design section, a local users group of subjects was tested in the camera car on two separate occasions; as novices, and after 6 weeks of use. These two groups were compared based on the objectives described in the four previous issue sections; driving performance, navigation performance, usability, and unsafe behavior.

This comparison was broken out as a separate issue in this plan for two reasons. As with the age and local versus renter group differences that follow, it was deemed particularly important for understanding TravTek use. As with local users versus renters, it was treated as a partial factor requiring a completely separate analytical procedure.

\section{ISSUE 6: DID DRIVING PERFORMANCE, NAVIGATION PERFORMANCE, AND DRIVING SAFETY VARY AS A FUNCTION OF AGE?}

Past research has shown that there are substantial driving performance and behavior differences between age groups.(19) Therefore, it was believed that such differences based upon driver age would be evident in this study as well. Three age groups were used to assess these differences.

To address this issue, the three age groups (16 through 18,35 through 45, and 60+) were compared based on the objectives described in issues 1 through 4 above. These age groups were selected to represent the extremes in age that could successfully be recruited.

This comparison was broken out as a separate issue because of the importance of understanding age-related driving performance and behavior differences. These are critical driving differences that are relevant to navigation system use, including risk assessment, judgment, reaction time, processing time, and vision and hearing sensitivity, just to name a few. Therefore, careful and comprehensive assessment of the three extreme age groups performance and behavior were accomplished as part of this test plan.

\section{ISSUE 7: DID DRIVING PERFORMANCE, NAVIGATION PERFORMANCE, AND DRIVING SAFETY VARY AS A FUNCTION OF TYPE OF USER (I.E. LOCAL OR VISITOR) AND AREA FAMILIARITY?}

One aspect that was addressed as part of the Camera Car Study was the extent to which performance and safety was affected by area familiarity. It was believed that area familiarity might give rise to additional differences in navigation behavior.

To appropriately address this issue, the two groups were compared based on the objectives described in the first four issue sections; driving performance, navigation performance, usability, and unsafe performance/behavior.

This comparison was broken out as a separate issue in this plan for two reasons. As with experience and age, it was deemed particularly important for understanding TravTek use. It was treated as a partial factor requiring a completely separate analytical procedure. 


\section{METHODS}

\section{DURATION OF TEST}

The camera car data collection took approximately 4 months to complete. The data reduction phase, including the classification of approximately 130,000 eye glances required an additional 6 months to complete.

\section{TEST CONFIGURATION}

The Camera Car Study evaluated the TravTek system effects on driver performance and behavior. This evaluation primarily focused on navigation and route following, since these constitute the most difficult route following.

The limited predrive portion of the study included intersection destination input via the TravTek touch screen. In general, the origin/destination pairs that were used for testing began and ended at intersections in residential neighborhoods. Subject input each destination at the beginning of the TravTek data run, or conducted trip planning with a paper map, or were given a textual direction list depending on the condition under test.

Once the drivers keyed in a destination (or found the destination on the paper map or direction list) and completed all desired route plannmg, they navigated from the origin to the destination. A total of six navigation test configurations were used for the Camera Car Study. Each driver/subject completed all six configuration drives in a counterbalanced order. These included:

- Symbolic turn-by-turn guidance map display with supplementary voice.

- Symbolic turn-by-turn guidance map display.

- Route map display with supplementary voice.

- Route map display.

- Paper textual direction list.

- Paper map (control condition).

The first four conditions enabled researchers to determine a rank ordering of the screen and/or voice configurations with respect to driving performance and system usability. The paper map and direction list conditions were included to provide navigation baseline conditions to which the TravTek system could be compared. Previous research has shown that any navigation technique requires greater attention demand than circumstances of nonnavigation driving.@ Inclusion of the two most common navigation techniques as control conditions provided an accurate way to measure TravTek costs and benefits. Note that no "normal driving only" baseline was included as a condition in the Camera Car Study. Although such a condition would provide some benefit in terms of assessing the differences between plain driving and navigating to a destination, it was deemed less important than the control conditions selected. It is recommended, however, that such a condition be included in future research projects of this nature if resources are available.

The following sections describe the TravTek visual and auditory display configurations that were tested as part of the Camera Car Study.

\section{Symbolic Guidance Display}

The symbolic guidance display (or turn-by-turn guidance screen) provided visual information about the next maneuver along the TravTek systems proposed route (see figure 
2). This display is described more fully in references twenty and twenty one. The guidance display represented the TravTek vehicles present position with an arrowhead. Guidance was in heading-up format. The next maneuver along a preselected route, and the relationship of the present position to that maneuver were displayed. Tic marks above the present position symbol represented $0.16 \mathrm{~km}(0.1 \mathrm{mi})$ to the maneuver point, and were displayed when the vehicle was within $1.4 \mathrm{~km}(0.9 \mathrm{mi})$ of the maneuver point. A large solid arrow indicated the direction of the maneuver. The name of the road that the vehicle was on, the name of the road at the next maneuver, distance to the maneuver, distance to the destination, and estimated time to the destination were displayed in text. Drivers had the option of selecting the route map or guidance displays via a swap-map switch on the steering wheel, but this switch was disabled for Camera Car Study drivers. When the TravTek navigation computer detected that the vehicle was off the planned route, the route map automatically displayed a banner across the lower portion of the screen that read "Off planned route?-OK new route?' This display enabled the driver to either accept a new route, ignore the route deviation (appropriate if the vehicle was on route as the system recovered correct position without operator intervention), return to the originally planned route, or correct the present position of the vehicle in the navigation system in conjunction with the "HOP" steering wheel button. When it detected that the vehicle was again on the planned route, the system automatically returned to the guidance display.

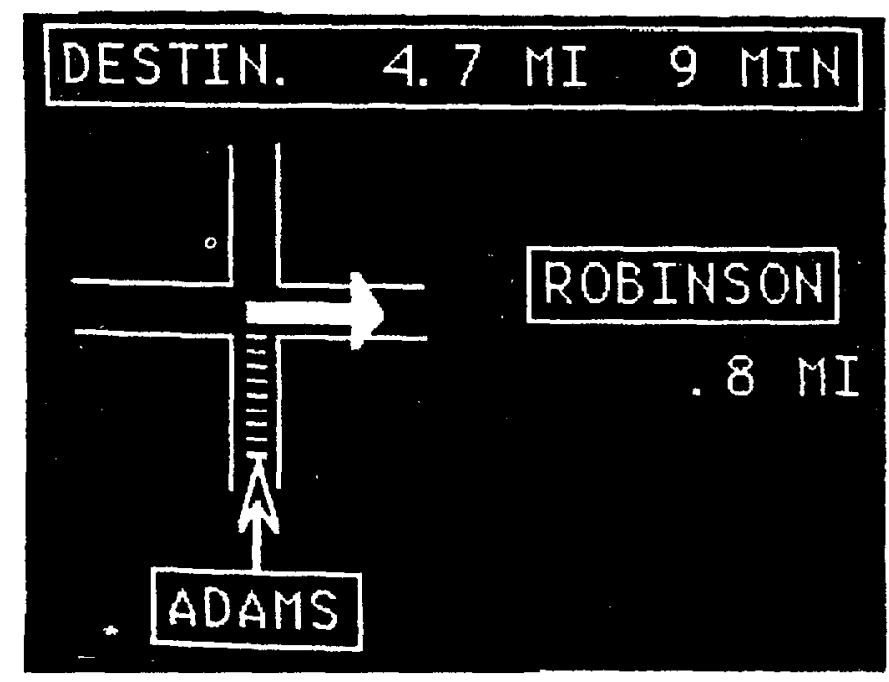

Figure 2. Guidance map display example.

\section{Route Map Display}

The moving-map display, or route map, provided a heading-up view on an electronic street map. The TravTek vehicles present position and the TravTek systems proposed route of travel were represented on the map. References twenty and twenty one describe the route map display. The TravTek vehicles present position was represented by an arrowhead. When the car was in PARK, the heading-up format could be changed to north-up. When the vehicle was stopped (it may be in gear), zoom-in and zoom-out option buttons were available. The zoom-in, zoom-out, and north-up options were selected via buttons displayed on the touch-sensitive screen. If selected, the north-up display automatically reverted to heading-up when the car was taken out of park. Once selected, the zoom scale remained the same until it was changed. Scales available with zoom were: $.20 \mathrm{~km}(1 / 8 \mathrm{mi})$, $.40 \mathrm{~km}(1 / 4 \mathrm{mi}), .81 \mathrm{~km}(1 / 2 \mathrm{mi}), 3.22 \mathrm{~km}(2 \mathrm{mi}), 8.05 \mathrm{~km}(5 \mathrm{mi}), 16.1 \mathrm{~km}(10 \mathrm{mi}), 32.2$ $\mathrm{km}(20 \mathrm{mi})$ and $64.4 \mathrm{~km}(40 \mathrm{mi})$. The map scale referred to the distance represented 
between the present position symbol and the top of the display. The present position arrowhead stayed approximately three-fourths of the distance from the top of the display. An example of a route-map display screen appears as figure 3.

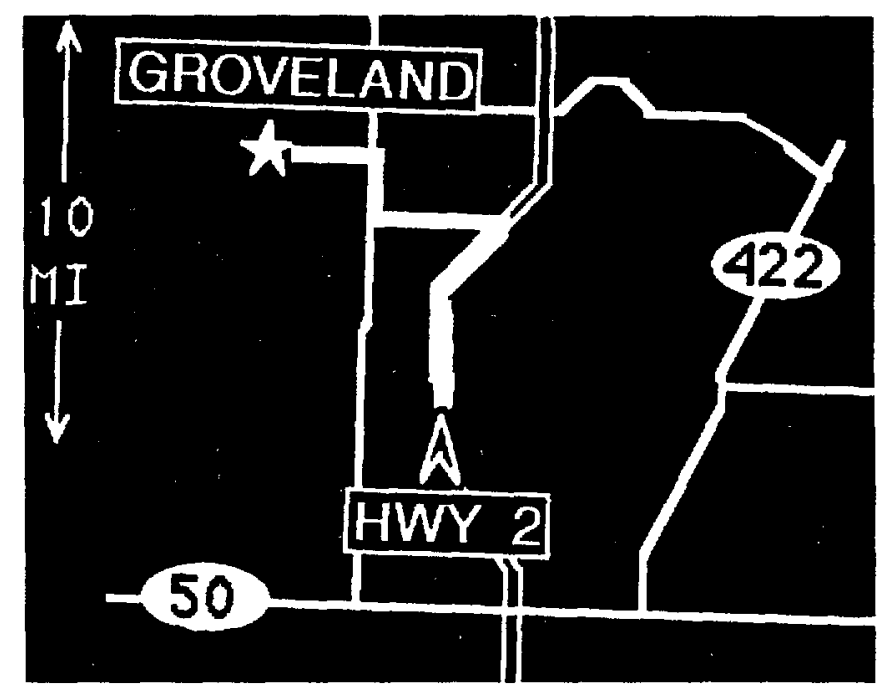

Figure 3. Route map display example,

\section{Aural Display Configurations}

Synthetic voice guidance was a feature of the TravTek system. The voice guidance system provided the following information:

- Starting points for planned routes.

- Alerts to maneuvers that were within $1.4 \mathrm{~km}(0.9 \mathrm{mi})$.

- Notices to prepare for maneuvers within $0.16 \mathrm{~km}(0.1 \mathrm{mi})$.

- Notices to execute maneuver.

- Description of the next maneuver (following completion of each maneuver).

- For a complex maneuver, a description of the maneuver following next maneuver (e.g., quick right then left).

- Alerts to being within $0.16 \mathrm{~km}(0.1 \mathrm{mi})$ of the destination.

- Alerts to being off the planned route.

- Alerts to the availability of better routes.

A steering wheel button toggled voice guidance on and off. The default condition was voice guidance on. Another button mounted on the steering wheel was used to request a present position voice message ("Where am I?"). These buttons (shown in figure 4) were not used in the camera car since the subject was instructed to navigate with a given configuration. These functions were, however, available to camera car local user subjects during the 6 weeks of TravTek use between their first and second camera car data collection runs.

The presence or absence of synthetic voice cueing were crossed with both of the visual display conditions creating the four TravTek test conditions described above.

The research design section of this report provides a detailed description of the experimental procedures utilized for the Camera Car Study. 


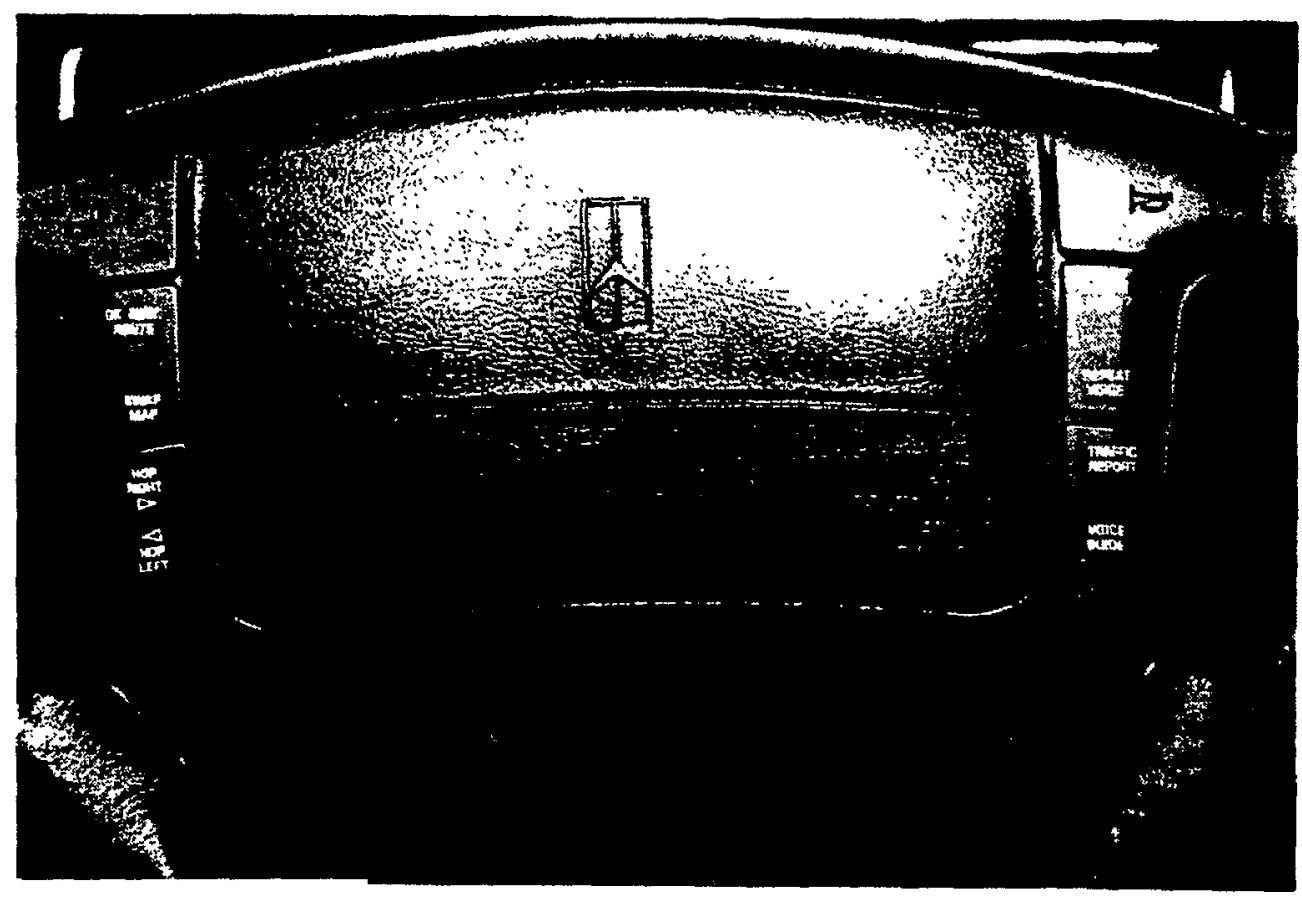

Figure 4. Steering wheel buttons.

\section{TEST CONDITIONS}

\section{Origin/Destination Pairs}

Evaluation of the display alternatives were conducted on roads in the Orlando, Florida area. Paid subjects traveled between origin/destination (O/D) pairs, which were defined by the experimenters. Each driver was tested with all display configurations. In order to counter the effects of learning, the displays could not be compared fairly with the same driver repeatedly traversing between the same origin and destination. Therefore, seven O/D pairs were used (one practice and six for data collection). Three of these O/D pairs were the same ones used in the TravTek Orlando Test Network Study - C2. For the case of the local users who drove twice in the study, an additional six O/D pairs were used. The pairs were selected to represent routes that equated, to the greatest extent possible, the following factors:

- Average travel time of 20 min during off-peak hours.

- Distance between origin and destination.

- Number of left and right turns.

- Distance on limited access roadways.

- Number of traffic sensors along route.

- Number of traffic control signals.

- Traffic volume.

- Average number of required stops.

- Congestion.

- Number of lanes.

- Direction of flow (i.e., one-way, two-way).

- Number of intersections.

- Number of access roads. 
Identification of $\mathrm{O} / \mathrm{D}$ pairs was complicated by the fact that only origin and destination were supplied to the participants. Although any route between the pairs was acceptable, the instructions recommended selection of a route with the shortest travel time. For the route map and guidance display configurations, the instructions directed subjects to select "fastest" from the TravTek route planning menu.(20) The "Navigation Mode" of TravTek was also used for camera car testing since it recommends identical routes consistently. "Navigation Plus" which will recommend route changes due to increased traffic congestion was not used so that subjects would receive identical routes and direction consistently.

To ensure that $\mathrm{O} / \mathrm{D}$ pairs were equated for routes that were commonly recommended by the TravTek system, a TravTek car that possessed all navigation capabilities was used during a dry run to assess candidate O/D pairs.

\section{Conducting Tests Using O/D Pairs}

The tests were conducted using the $12 \mathrm{O} / \mathrm{D}$ pairs as described above. In addition, six short, 5-min practice O/D's were driven (one for each experimental condition).

Each O/D pair selected required a minimum of seven turns (regardless of route selected) to reach a destination. This number precluded simple memorization of a planned route during the predrive phases of the data runs.

During a data run, the traffic density was classified by the in-vehicle experimenter to account for variance in driving task difficulty. However, because the O/D pairs all began and ended in residential areas and because tourist attractions and traffic incidents made the nature of off-peak traffic somewhat unpredictable, some variability in traffic density was inevitable for this experiment. To properly account for this variability, the in-vehicle experimenter classified the traffic density as low, moderate, or high (via a custom experimental control panel) based on pre-specified criteria provided by the Department of Transportation (DOT).

All data collection for this study occurred during daylight hours.

\section{RESEARCH DESIGN}

To address the issues described in the previous section, the Camera Car Study was conducted in two separate phases with two separate user groups. The first phase of the experimentation consisted of visitors to the Orlando area. The second phase of the experiment consisted of local users. To study the effects of familiarity and practice, the local users were tested on two separate occasions; when they were first introduced to the car, and after 6 weeks of daily use.

For each of the two experimental phases the research design was a mixed factor, complete factorial design. The two factors were navigation technique and age. In addition to the above factors, gender, roadway type, and traffic density were used as matching variables.

Note that in addition to the independent variables listed above, analytical comparisons were made between each phase (i.e., user group), as well as between the first time and returning local users to study practice and experience effects. Therefore, these comparisons also constitute manipulated independent variables. Each of the manipulated or matching variables is described in the following paragraphs. 


\section{Navigation Technique}

Navigation technique was a within-subject factor. Six different navigation techniques were assessed. The TravTek navigation techniques included:

- Turn-by-turn guidance screens with voice guidance.

- Turn-by-turn guidance screens without voice guidance.

- A route map with voice guidance.

- A route map without voice guidance.

- Textual paper direction list.

- A conventional paper map.

The presentation orders of the navigation techniques were counterbalanced to minimize order effects.

\section{Paper Map And Textual Direction List}

The purpose of including paper map and direction list conditions was to provide an accurate navigation baseline condition for TravTek system comparison. It is certain that any navigation technique will require greater attention demand than circumstances of nonnavigation driving. A comparison of available alternative navigation techniques was performed to determine the cost and effectiveness of such a system.

\section{Age}

Subjects were selected and divided into three distinct age groups. Previous studies have shown that older drivers behave differently than younger drivers while performing navigation system related tasks both on the road and in the laboratory.@) Due to the accident rates for populations of differing ages presented in the test configuration section, the three groups of phase 1 (visitors) consisted of drivers ages 16 to 18,35 to 45 and $65+$. For phase 2, two age groups were tested, 35 to 45 and 65+. Drivers under the age of 25 could not participate in the local user portion of the study due to Avis insurance requirements.

\section{Roadway Type}

Roadway type was a within-subject factor. Four differing types of roadway configurations (i.e., residential streets, two-lane arterial, multilane arterial, and freeway) were presented in each O/D. The manipulation of this factor allowed for the assessment of navigation and driving performance under normally occurring attention demand variations induced by changes in road type.

\section{Gender}

Both genders were used as drivers in this research. Although few studies have shown driving performance differences between genders, driving behavior and risk acceptance has been shown to vary in selected circumstances. Gender, in this experiment, served only as a matching variable and did not undergo statistical analysis.

\section{Traffic Density}

The density of surrounding traffic was determined and classified by the in-vehicle experimenter as low, medium, or high. The criteria for this classification scheme were 
provided by the DOT via photographs of varying traffic densities. As with road type, this classification reflects circumstances which create variance in driving task demand.

\section{Subject Type and Experience}

Eighteen visitors (six per age group) and 12 local users (6 per age group) participated in this study. The subjects were selected based on age and gender as discussed above. The visitors participated in a single set of navigation runs. The local users participated in two sets of navigation runs. The first set was performed while the local users were system novices. For this condition, local users received the same instruction, practice, and experimental treatment as the visitors. This allowed comparisons between the two types of user groups. The second set of runs occurred after the local users had used the vehicles daily for 6 weeks, Comparison between the two local user navigation runs allows analysis of practice and experience effects while using the TravTek system. A manipulation check was performed for each local user to determine the actual use of the TravTek configurations during the 6-week period.

\section{Training Tools}

The training tools associated with the Camera Car Study included brochures, a training video, and guided practice. These materials and brochures did not differ from those that were provided to subjects in other studies. It should be noted that participants in the Camera Car Study received their training at the time of, or before, their participation in the Local User Study.

\section{RESOURCE DESCRIPTION}

\section{Unit of Analysis}

The unit of analysis for the Camera Car Study was individual drivers. The criteria for choosing these drivers was that they were unfamiliar with the O/D. Otherwise, the navigation data would be confounded. The 18 visitors were recruited via local advertisement. The 12 local users were high mileage Orlando drivers recruited by the contractor.

High mileage drivers within the Orlando traffic network were selected for participation in the local user study. An attempt was made to find drivers who had to daily navigate to unfamiliar areas. All attempts were made to recruit drivers in the selected age categories that met the high mileage criteria. However, if the mileage requirements could not be met, they were relaxed to some degree to maintain the age groups described above.

\section{Camera Car Equipment Description}

The camera car consisted of a basic TravTek vehicle to which multiple sensors, event recording and data recording instrumentation had been added. The camera car data collection system provided the means to collect, record and reduce a number of data items valuable for Human Factors and Safety Test and Evaluation of the TravTek system. These data items included measures of attention demand, measures of navigation performance, safety-related incidents and subjective opinions of the driver/subjects. The system consisted of video cameras to record pertinent event and eye movement data, an experimenter control panel to record incident classification data, sensors for the detection of variations in driving performance and behavior, and a custom analog to digital interface and computer to log the data in the form required for analysis. The camera car hardware and 
software design and integration were conducted by General Motors Research Laboratories. A schematic of the camera car appears as figure 5 .

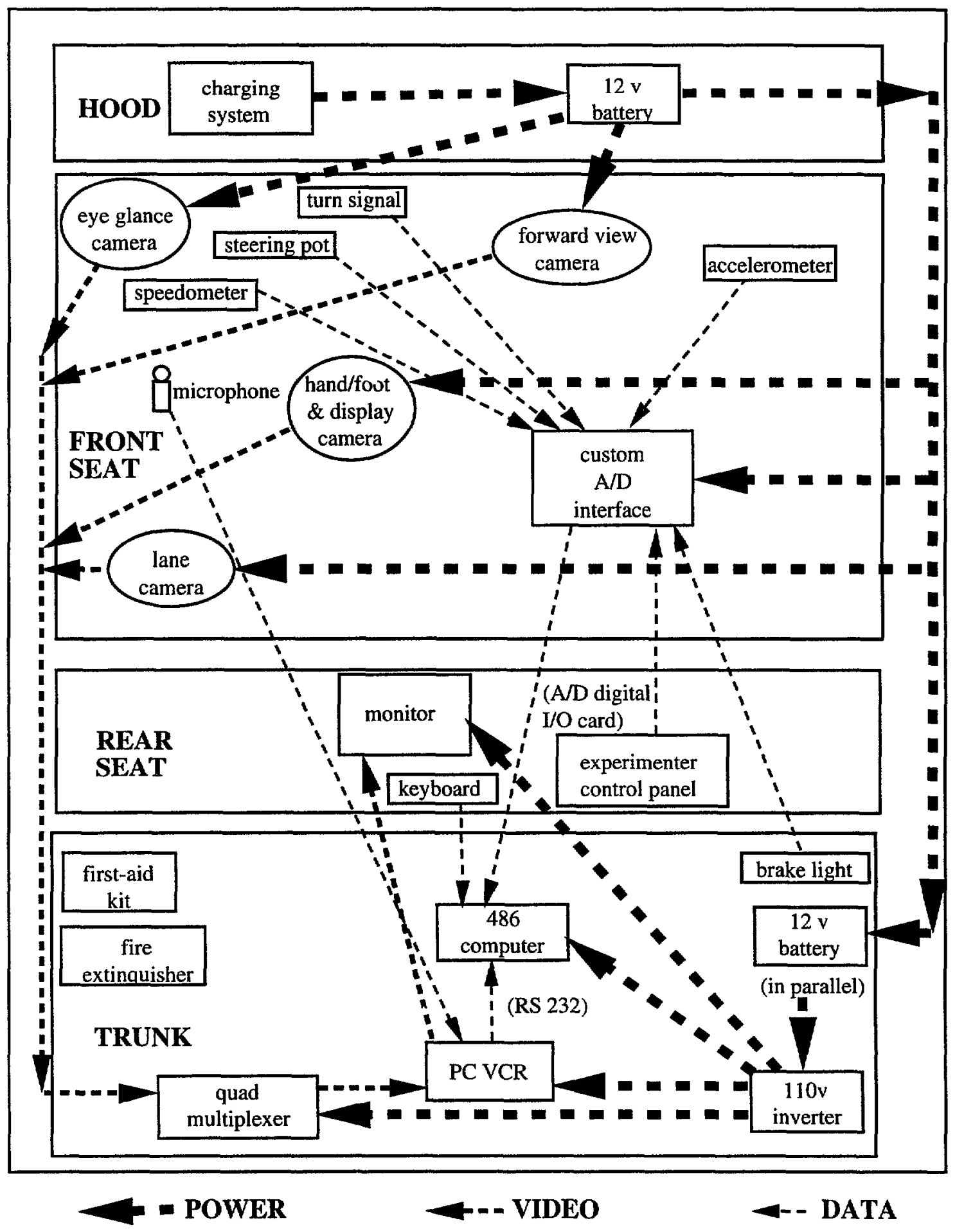

Figure 5. Camera car schematic diagram.

The camera car data collection system allowed the collection and storage of a number of measures described below. In general, the system provided the capability to store data on 
the data collection computer in the form of a line of numerical data every $0.1 \mathrm{~s}$ during a data run. The videotape of the four camera views was time stamped and synchronized with the computer data stream so that post-test data reduction and data set merging could occur in the laboratory. All data collection records were time stamped to an accuracy of $+/-0.1 \mathrm{~s}$.

\section{Eye Glance Camera}

The eye glance camera was used to monitor eye movements for a variety of driver subjects under a variety of circumstances. A photograph showing the eye glance camera (just above and on the left side of the rear view mirror) appears as figure 6. It had a wide enough fieldof-view to accommodate drivers of differing heights and seating positions. The view of the subject's eyes were clear, in focus, sufficiently large and automatically light adjusted (in daylight conditions) to allow easy eye movement classification in the laboratory. The eye glance camera was placed above the center rear view mirror and did not obscure the driver's view of any part of the driven roadway. The camera recorded drivers' head and eye positions so that eyes-off-the-road time, glance frequency, glance duration, and invehicle visual targets could be ascertained.

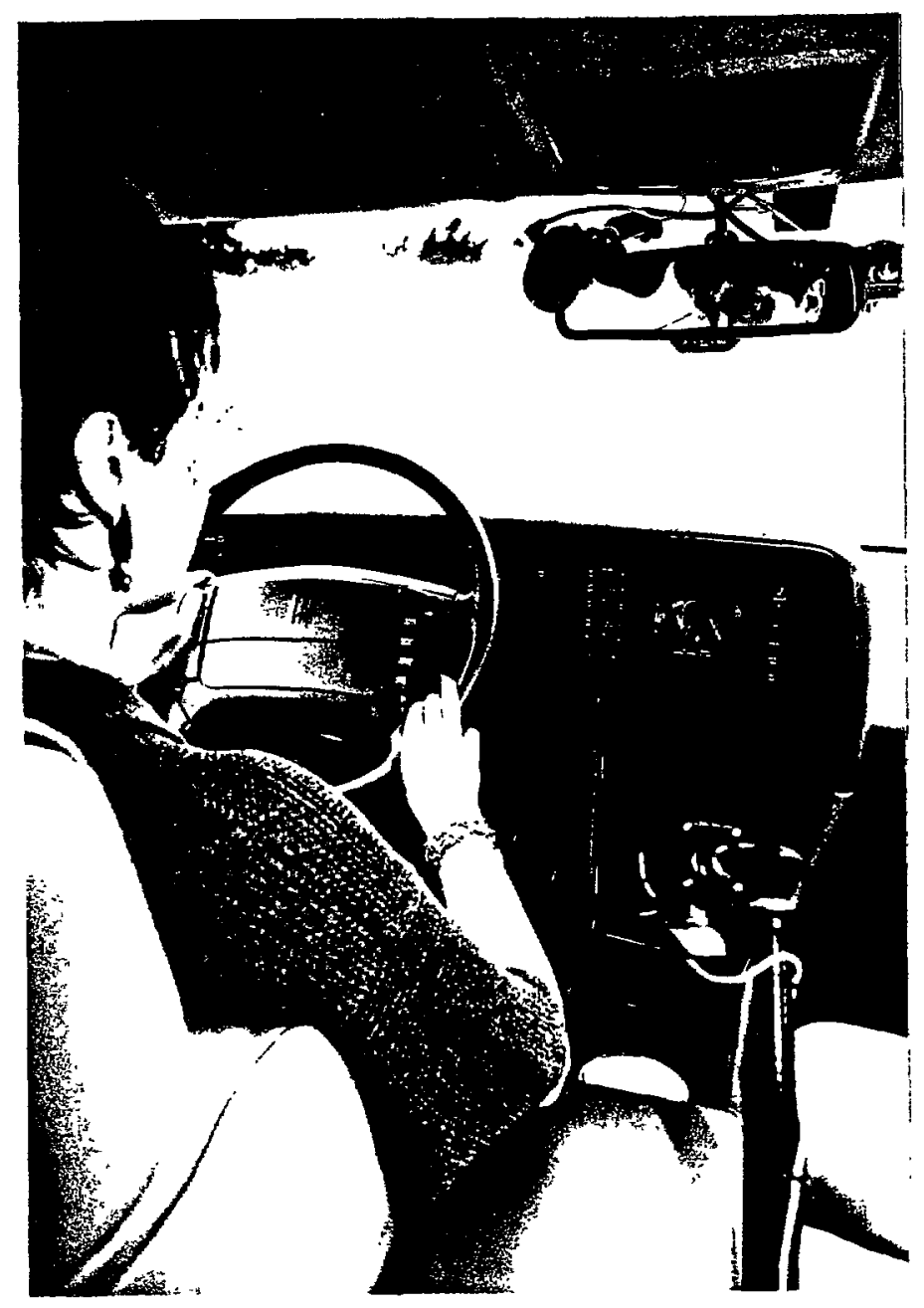

Figure 6. Camera car photograph of the TravTek display, controls, and selected cameras. 


\section{Forward View Camera}

The forward view camera provided a wide view of the forward roadway without substantial distortion of the recorded view. The camera had an auto-iris and provided a high quality picture in all but the most severe daylight glare conditions. The forward view camera was located above the center rear view mirror (partially visible in figure 6) and did not obscure any part of the subject's view of the roadway. It served the purpose of collecting relevant data from the forward scene (e.g. traffic density, signs and markers and headway).

\section{Vehicle Information Center/Steering Wheel Camera}

This camera provided a single view of the steering wheel including the driver's hands during normal control movements, as well as a view of the Vehicle Information Center in the camera car. The camera provided an accurate depiction of the information of interest under differing daylight lighting and glare conditions. The camera was positioned to provide an over-the-shoulder view as shown in figure 6.

\section{Lane Tracking Camera}

The lane tracking camera provided a direct view of the front left tire and the centerline of the roadway. A photograph of the camera car with the lane tracking camera mounted just below the left rear view mirror is shown in figure 7. The lane tracking camera was equipped with a very wide angle lens so that some additional information could be gained from the surrounding traffic scene. This camera was also equipped with an auto-iris function so that high quality images could be maintained under the majority of daylight lighting conditions.

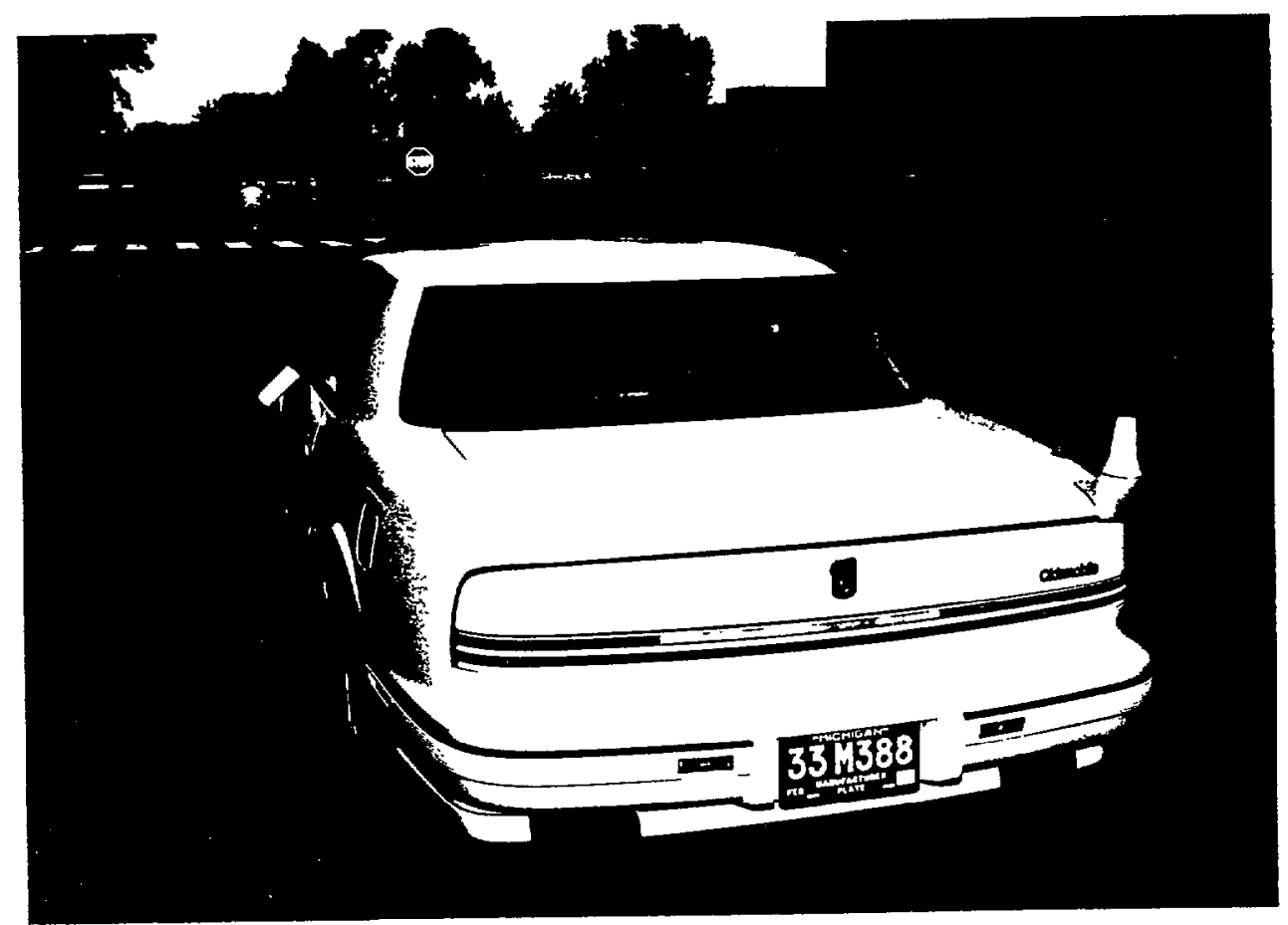

Figure 7. Camera car photograph showing the position of the lane tracking camera. 


\section{Lateral and Longitudinal Accelerometer}

An X/Y accelerometer provided acceleration readings in the lateral and longitudinal plane of the vehicle. The accelerometers provided values for vehicle acceleration and deceleration up to and including hard braking behavior as well as intense turning. The sensor provide a signal that was read by the A-D interface at 10 times/s. The multiplane accelerometer, in conjunction with information inputs from the speedometer and brake light sensor, provided measures of lateral and longitudinal accelerations, velocity trends (vehicle speed maintenance and speed variance), brake actuations and hard braking maneuvers.

\section{Data Collection Computer}

The data collection computer provided reliable data collection, manipulation and hard drive storage under conditions present in a vehicle environment. The data collection computer was rack-mounted in the trunk of the camera car as shown in figure 8 . The data collection computer is mounted in the bottom of the rack shown in the figure. The computer also provided reliable file copy capability in a parked vehicle. The computer had a standard QWERTY keyboard and a $22.86 \mathrm{~mm}$ (9-in) diagonal black and white monitor that were mounted in the experimenter's station in the rear seat of the camera car (figure 9). The computer had an Analog to Digital capability. Computer memory and processing requirements were equivalent to a 486 with 8 megabytes RAM and a 80 megabyte hard drive.

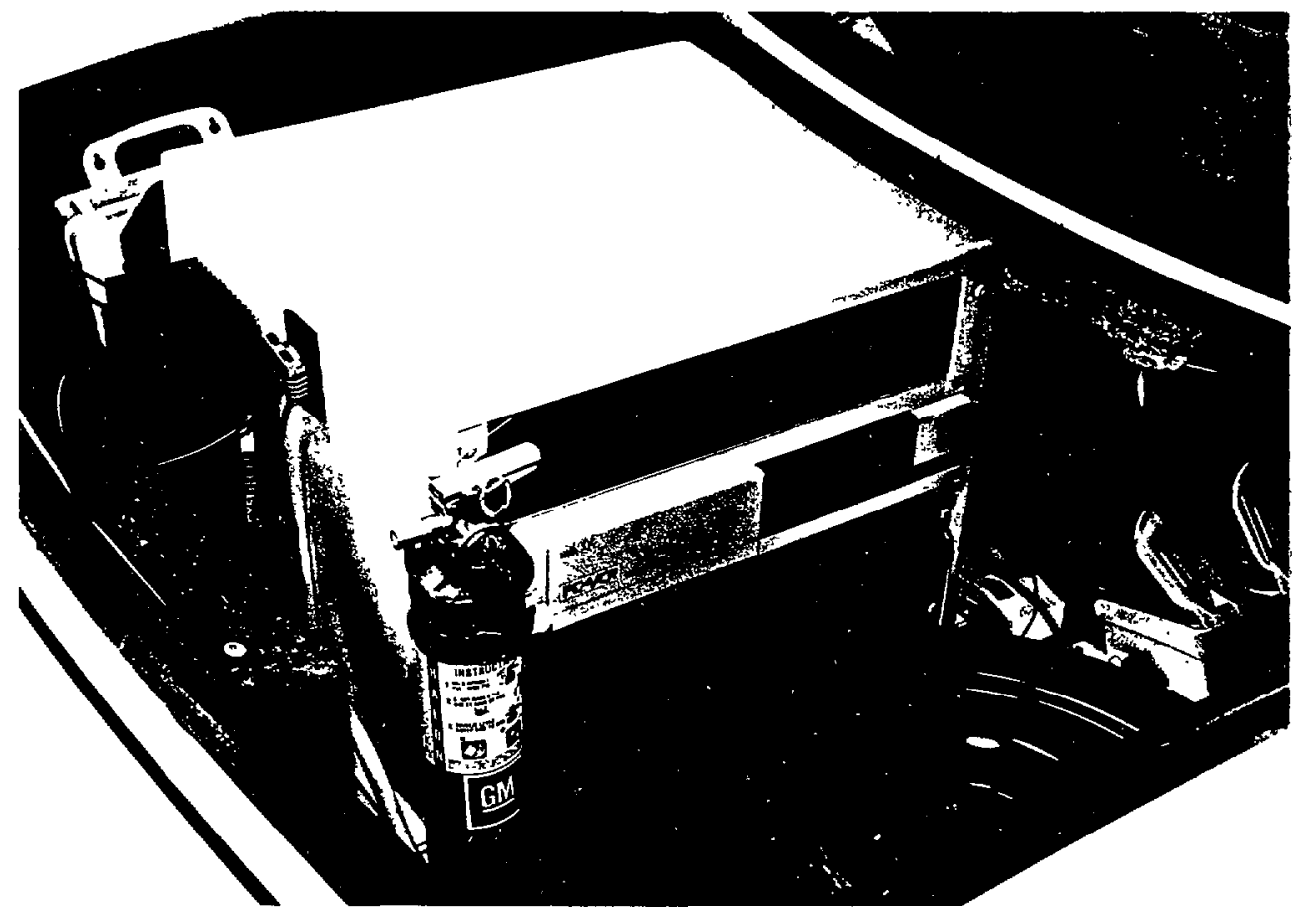

Figure 8. Camera car trunk mounted equipment.

\section{Experimenter Control Panel}

A custom experimenter control panel was provided in the rear seat of the camera car (figure 9). The control panel consisted of a panel of hard buttons and configured such that positive activation of functions was feasible without inadvertent activation in a moving vehicle. The 
control panel allowed input of roadway type, traffic density, route planning time, travel start and stop times, and driving and navigation errors.

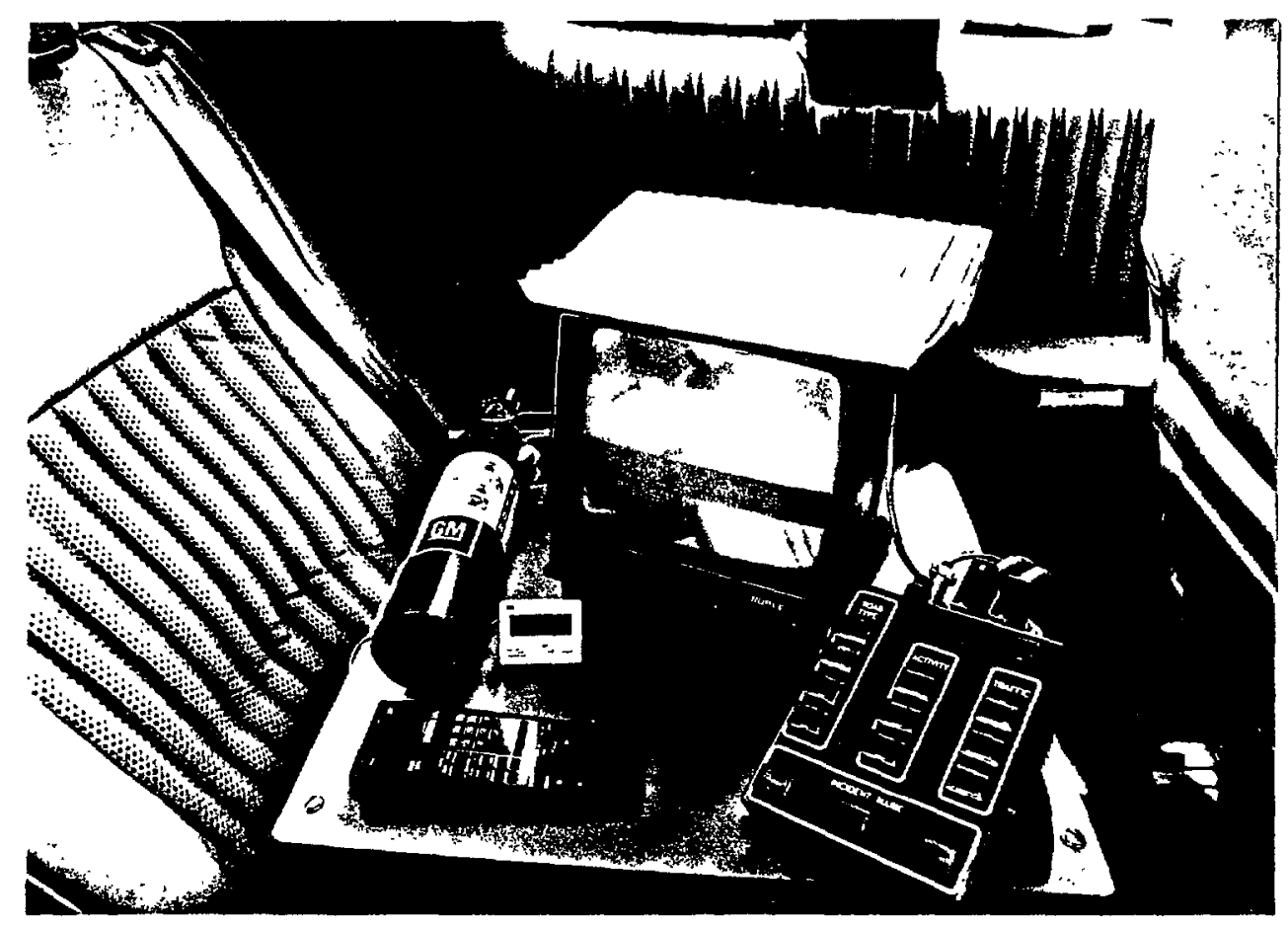

Figure 9. Camera car experimenter station in the rear seat.

\section{Video/Sensor/Experimenter Control Panel Interface}

A custom interface was constructed to integrate the data information from the experimenter control panel, driving performance sensors, brake light, turn signal, and speedometer with the data collection computer. In addition, the interface provided a means to accurately read and $\log$ the time stamp from the PC-VCR to an accuracy of $+/-0.1 \mathrm{~s}$. The time stamp was coded so that a precise location could be synchronized from any of the videotaped records to the computer data record for post-test laboratory reduction and file integration.

\section{Audio Data Collection System}

An audio track of the videotape of the experiment contained the commentary of the experimenter, driver/subjects and TravTek voice system. The stored audio data were clearly audible in a quiet laboratory environment.

\section{Multiplexer}

A quad multiplexer was provided to integrate the four camera views including the time stamp onto a single videotape. The multiplexer was mounted in the trunk of the camera car (figure 8, top unit in the equipment rack).

\section{PC-VCR}

A PC-VCR was provided to send an accurate time stamp to the data collection computer, as well as display the time stamp continuously on the four multiplexed view of the videotape 
(figure 8, middle unit in the equipment rack). In addition, the PC-VCR had the capability to read and mark event data provided by the data collection computer and performhighspeed searches for event marks provided. The PC-VCR was a Super VHS (SVHS) format so that each multiplexed camera view had 200 horizontal lines of resolution.

\section{Sensors}

The camera car steering wheel, speedometer and brake pedal were instrumented. The steering wheel sensor provided steering position data accurate to within $+/-1$ degree. The recorded driver steering inputs were used to provide frequency and magnitude measures and steering reversals. The brake signal provided an indication of whether the brake was on or off.

\section{Safety Measures}

The following safety measures were provided as part of the camera car system. Such measures helped to minimize the driver/subject risk during the conduct of the experiments.

1. All data collection equipment was mounted such that, to the greatest extent possible, it did not pose a hazard to the driver in any foreseeable case.

2. A driver-side air bag was provided in the camera car.

3. A fire extinguisher and first-aid kit were provided in a secured location in the passenger compartment of the experimental vehicle.

4. None of the data collection equipment interfered with any part of the driver's normal field of view present in the automobile.

5. A trained in-vehicle experimenter was in the vehicle at all times, Emergency protocol were established prior to testing.

\section{Human Resources}

The human resources that were used to conduct the Camera Car Study included a task' leader (to lead the Camera Car Study and team, and to ensure that all experimental requirements were met), an experimental technician (to serve as a single, trained human factors engineer and the in-vehicle experimenter), a senior research analyst (to, assist in the overall conduct of the Camera Car Study with particular emphasis on the video coding/analysis), and research assistants (who were responsible for data entry and coding).

\section{Data Collection Instruments and Sources}

The data collection instruments and sources for the Camera Car Study included the following:

- Four strategically located color video cameras.

- PC-VCR.

- Multiplane accelerometer.

- Steering potentiometer.

- Audio recorder.

- Experimental control panel.

- In-vehicle computers (including a 486 data collection computer). 
- Brake pedal activation sensor.

- Subjective workload measurement technique.

- Experimenter notes.

- Subject screening tests.

- Pre- and post-test questionnaires and debrief.

These hardware instruments listed above are described in detail in the equipment subsection. The screening pretests included a map skills test, vision screen, and audition screen. The map skills screening consisted of tests from the Kit of Factor-Referenced Cognitive Tests 1976, a self-assessment questionnaire and some map performance measures. (23)

The building memory, map memory, card rotation, and map planning tests from the Kit of Factor-References tests were administered to participants prior to in-vehicle data collection, These tests were selected for inclusion because they were likely to reflect subject qualities related to map skill, were easily administered and scored (less than $6 \mathrm{~mm} / \mathrm{test}$ ), and there was literature relating performance on these tests to other cognitive and performance measures. These tests were used to ensure that subject groups were roughly equivalent in map skill but were not analyzed as part of the results of this report.

The purpose of the vision and audition screenings was to provide data to aid in the interpretation of results, especially driver age results. Drivers with less than 20/40 acuity with high contrast stimuli were excluded, as were drivers who could not adjust the voice cueing volume sufficiently to understand voice messages.

\section{PROCEDURES}

\section{Subject Screening}

Potential subjects had to pass an informal hearing and formal vision test (with 20/40 near vision and 20/40 far vision). They also filled out the questionnaire of familiarity with Orlando land marks to determine geographical awareness. If the potential subjects were unfamiliar with the Orlando area (in the case of the visitors group), if they passed the hearing and vision tests, and if they had no conditions which might interfere with their driving ability, they were considered as qualified to participate in this study.

\section{Instructions and Informed Consent}

Drivers were given the standard introductory material used in all TravTek studies to familiarize themselves with the system. In addition, the subjects were given an informed consent form. Both informed consent forms for visitors and local drivers are shown in appendixes A and B. Driver questions pertaining to the reading material were answered by experimenters and drivers were instructed to sign an informed consent form if they wished to participate in the study.

\section{Training}

For the Local User Novice/Expert Study, training and orientation of drivers were performed as part of their participation in the Local User Study. The following description constituted the training program for the Visitors' Study.

Training and orientation of these subjects were provided at the time of their participation. Drivers were shown a AAA video that was used in the OTNS. They were given reading 
materials (e.g., a condensed version of the TravTek manual). Then the drivers were shown and trained on an actual TravTek system. Once the drivers felt comfortable with how to use the system and passed a verbal test of TravTek features, the six practice runs took place. For each O/D training session, the driver used one of the six main presentation arrangements (symbolic guidance map display with supplementary voice, route map display with supplementary voice, paper map, route map display, symbolic guidance map display, and direction list) for approximately 5 min each. During each run, the driver programmed in and drove to a destination. Help was provided when warranted. Subjects were also instructed how to give the subjective workload ratings that would be periodically requested from the experimenter and gave them during the six practice runs. After completion of the practice run, the experiment and data collection commenced.

\section{Data Collection}

For both phases of the study, an experimenter sat in the right-rear seat with a data recording workstation. This workstation included a mini-QWERTY keyboard, monitor, and experimenter control panel.

Subjects were instructed to think and read aloud as they navigated in order to provide greater insight into subjects use of TravTek. The subjects were then given their first destination printed on a card and told to drive the route as efficiently as possible. Once the driver was handed the card, the experimenter depressed the "begin plan" button located on the experimental control panel, and the driver planned and/or entered in the destination. When the driver put the car in DRIVE, the experimenter depressed the "begin trip" and the corresponding road type and traffic density buttons located on the experimental control panel. A workload rating was then requested from the driver for the planning portion of the drive.

Along the route, the experimenter monitored the density of surrounding traffic and changes in roadway type. Traffic density was coded as either low (fewer than 6 vehicles within sight distance forward of the camera car), medium ( 6 to 12 vehicles within the forward sight distance) or high (greater than 12 vehicles within the forward sight distance).

Roadway type was classified as residential street, two lane arterial, multilane arterial, and freeway. The experimenter, upon a change in either roadway type or traffic density, depressed the corresponding button on the experimental control panel. Subjective workload ratings were also requested during every portion of the drive. When a driver switched road type, they were requested to provide a subjective workload rating. Ratings were also requested when subjects deviated from the planned route.

The experimenter depressed the "event mark" button during a data run when any of the following occurred:

- Any trigger event associated with unsafe driving (as discussed in the unsafe driving behavior section - issue 4).

- An unexpected lane change by another driver.

- An unexpected side road pullout by another driver.

- An unexpected slowdown or stop by another driver.

- An unexpected pedestrian roadway entry.

- An intersection approach.

- A lane deviation by the driver.

- A planned lane change by the driver.

- A change in traffic signal status.

- A predictable traffic slowdown or change. 
The experimenter was also requested to depress a "navigation error" button when navigation-related errors such as missed turns and off-route circumstances occurred. The purpose of this button was to mark navigation events as opposed to driving performance events.

Once the drivers reached the destination, the experimenter depressed the "end trip" button on the experimenter control panel.

At the end of the last O/D pair run, the drivers were asked to answer the post-test questionnaires and were debriefed.

\section{Data Management}

The in-vehicle data were backed-up on diskette following each O/D data collection segment. Upon completion of the data run, the data were entered into the TravTek Camera Car Data Base in its raw form.

\section{DEVIATIONS, EXCEPTIONS AND ADDITIONS TO THE CAMERA CAR TEST PLAN}

In general, there were few deviations, exceptions, and deletions to the TravTek camera car Test Plan that occurred during the process of data collection, reduction, or analysis. Those changes that were made to the original protocols are described below.

Several changes to the original plan were made in the field, so that procedures for the Camera Car Study were consistent with the testing protocol from other TravTek tests. For consistency across studies, the camera car experimental procedures were identical to those used in other applicable studies.

Although map skill information was collected for the Camera Car Study, it was not addressed analytically, due to the small sample sizes used. The data were used as a screening device to ensure that the subjects varied in skill and did not represent the extremes of the population. However, analyses contrasting map skill differences could not be accomplished. An additional navigational skill test, the "how good is your sense of direction" test, was excluded based on experience in the other TravTek studies.

The test plan outlined a 75+ age group for both the visitors' and local users' studies. It turned out not to be feasible to recruit drivers over 75 to participate in the study who met the remaining criteria. Therefore, the oldest age group requirement was relaxed to $65+$.

When local users were recruited, it was important to find high mileage drivers as well as low geographical familiarity. High mileage drivers were found, but not with low geographical familiarity. These drivers, due to their inherent experience, generally knew the Orlando area well. None, however, were familiar with the residential neighborhoods that made up the $\mathrm{O} / \mathrm{D}$ pairs.

For practice, the subjects drove each navigational configuration in a 5 min practice O/D. These practice drives were performed in the same manner as the other contractor's TravTek studies.

In a hardware change, microphones were built into the interior of the camera car so that both the experimenter and the subject did not have to wear a microphone. 
In order to correspond to the contractor's protocol and make the street name spelling task easier, the experimenter handed the subject a 4-by-5 card with the destination of the next drive printed on it. Originally, the experimenter verbally told the subject the destination.

For a variety of reasons, MOP's were added, deleted, and modified during the data collection and reduction process. In general, the number of additions met or exceeded the number of deletions, and each issue had a number of valid MOP's to assess the hypotheses of interest. The additions included the following:

- Number of steering wheel reversals greater than 6 degrees.

- Average vehicle velocity.

- Variance in lateral acceleration.

- Mean negative longitudinal acceleration.

- Variance in longitudinal and negative longitudinal acceleration.

- Number and length of brake applications.

- Number and length of glances to the navigation aids.

- Number and length of glances to the roadway environment.

- Subjective measures of driver workload.

- Number of times lost.

- Indecision about turn correctness.

- Missed turns.

- Anecdotal usability observations from the in-vehicle experimenter.

The above MOP's were not originally proposed to be collected in the camera car Test Plan. The measures were added to the list of performance measures because each provided at least some unique insight into performance, and it was determined in the data analysis process that the measures were easily derived.

In the cases listed below, proposed MOP's were not collected and/or not analyzed:

- Route replanning time.

- Travel distance.

- Time off-route.

- Driver perception of configuration contribution to navigation performance.

- Perceived configuration effectiveness.

- Number of route planning errors.

- Number of system interactions.

- Circumstances where the driver had three or more successive glances to the navigation system.

In the cases of route replanning time, travel distance, time off-route, number of routeplanning errors, and number of system interactions, the original plan was to extract these data from the TravTek data log. This process proved to be technically difficult and, in some cases, less meaningful or reliable than originally anticipated. Therefore, given that other measures could be more easily obtained and that all issues were addressed with a number of valid MOP's, these data were not analyzed.

In the cases involving driver perceptions, the questionnaire responses for the camera car subjects were not analyzed as a group, due to the small Camera Car Study sample size. However, these data Were combined with questionnaire data from the TravTek Visitors' and Local Users' Studies to increase the number of respondents in those efforts.

In the case where three or more successive glances were to be analyzed, criteria could not be effectively developed to determine an operational definition of a successive glance. That 
is, since the subjects were not instructed to perform a specific task requiring a response, the number of successive glances to obtain a given item of information could not be effectively determined.

A limitation in the data analysis was the inability to quantify unplanned speed variation and dangerously close headway. Speed variation was collected with other computer data measures; however, there was a lot of noise associated with this data stream. It would be difficult to distinguish incidents of speed variation due to traffic, and incidents due to increased attention demand caused by the navigational condition. The experimenter riding in the camera car was the best judge of the extreme cases of slow speed, and these incidents were in fact collected. Similarly, it was originally planned to collect unsafe headway data by reviewing the videotape. However, this proved to be too difficult. Only extreme values were flagged and analyzed based on the in-vehicle experimenters opinion.

Another factor was the experimenter's workload. Due to the variety of responsibilities, occasionally a safety relevant event was missed. To compensate, a more stringent analysis of the videotape was performed by a second evaluator. The full duration of each subject run was reanalyzed in all cases in order to find any missed triggers or incorrectly classified events.

The test plan method also changed the source of abrupt lateral and braking trigger points. The test plan proposed that acceleration exceeding three standard deviations higher than the mean lateral and longitudinal acceleration as a trigger event be analyzed in the videotape. This method proved difficult to implement due to the sheer volume of data and an inability to differentiate between unsafe events and normal turning behavior. A safe driver would still have events that would need to be analyzed using the standard deviation method of identifying possible acceleration events. To compensate for this, a value of $3.9 \mathrm{~m} / \mathrm{s} 2(0.4$ $\mathrm{g}$ ) in either lateral or longitudinal acceleration was used as the trigger point. This was still conservative enough to catch all of the serious safety-related errors and was easier to implement than the standard deviation derived criterion. 


\section{DATA ANALYSIS OVERVIEW}

Experimental variables associated with the Camera Car Study phases are described in the research design section. The analyses planned for the Camera Car Study generally included the following:

- Descriptive Statistics - Descriptive statistics were computed for the measures of performance described in each of the issues sections. These statistics included measures of central tendency (mean, mode, median), measures of variability (standard deviation and range), and various distributions and graphs as appropriate. For the unsafe driving behavior section - issue 4, many of the measures were frequency counts of driver errors and near misses. For these cases, simple sums of occurrences are reported.

- Inferential Statistics - Inferential statistics included univariate analyses of variance (ANOVA's) and studentized maximum modulus (SMM's) post-hoc comparisons.(24) ANOVA's were conducted utilizing the SAS General Linear Models procedure since cell sizes were rarely equivalent. Multivariate-ANOVA's (MANOVA's) were not performed. MANOVA's often exhibit an increase in type II error for repeated measures designs. Fortunately, the majority of the univariate ANOVA's had p values that were well below the $p<0.05$ criterion value for significance utilized in this research. The reader is cautioned, however, against placing too much weight on a single ANOVA with a $p$-value approaching $p=0.05$ due to the possibility of a type I error. The results described in this report should be interpreted by looking for supporting evidence across of performance measures collected. The SMM post-hoc test was selected because it is a conservative test that holds the experiment-wise and comparison-wise type I error rates constant.

Non-parametric statistics were not conducted for the frequency data generated by the eye glance data, safety near-miss, and error data for three reasons. First, the number of occurrences in many cases were so large that almost any comparisons of meaningful differences will be statistically significant. Second, typical analyses evaluated differences between at least 6 , and as many as 48 , different conditions across the conditions of interest. Therefore, many different contrasts would be required to assess pairwise comparisons of interest. Since no non-parametric post-hoc test exists which holds type I error constant, the number of comparisons would either result in type I or type II errors depending on the strategy employed. Third, the comparisons of interest are repeated measures or mixed factors. These cases complicate the analysis of frequency data due to the violation of the independence assumption required for many chi-square based tests.

Fortunately, most of the differences analyzed for these data are large enough, and the measures typically face-valid enough, that statistical inference is not required. However, the reader is advised not to treat smaller frequency differences between conditions as statistically significant, particularly in the cases of smaller numbers of observation. The results section stresses those cases where caution is advised when interpreting results. The Camera Car Study results, in addition to providing stand-alone results, provided input into Study E2 - Safety Evaluation. The study results provided an assessment of whether the TravTek configurations put drivers at risk in comparison to other available means of navigation. This risk assessment data included indices of driving task intrusion, detailed analyses of trigger events pre-determined to represent unsafe behavior, and driver safety observations of using the TravTek system. These data, when combined with data from other field studies (including accident data, driver feelings of safety from questionnaires, and safety benefits such as routing traffic around incidents) were used to comprehensively evaluate the safety costs and benefits of the TravTek system. 


\section{RESULTS AND DISCUSSION OF ISSUES}

The results of the Camera Car Study are presented in the following sections according to the seven issues (driving task intrusion, navigation performance, usability, safety, user experience, age, and area familiarity) described in the previous sections. To facilitate the usability of this document, each issue is treated completely as a "stand-alone" section. As part of this stand-alone structure, discussions of results are included in each of the issues sections. Following these sections, a general discussion and conclusions section summarizes findings across the entire Camera Car Study.

\section{ISSUE 1: WHICH NAVIGATION CONFIGURATION(S) RESULTED IN THE LEAST DRIVING TASK INTRUSION?}

One of the primary purposes of the camera car and Camera Car Study was to provide a detailed evaluation of driving performance. Therefore, driving performance has been broken down into various performance measures as described below. Note, as was previously defined, degradation in driving performance means various navigation configurations result in driving task intrusion.

\section{Driver Eye Glance Behavior}

With respect to driving task intrusion, measures of eye scanning behavior provide an inherently valid measure since driving information is primarily gained from vision. The camera car provided the capability to categorize driver eye glances to 14 different locations. These locations included:

- Forward roadway (center).

- Left roadway.

- Right roadway.

- Left-hand check (side window).

- Right-hand check (side window).

- Dashboard.

- TravTek display, map or direction list.

- Steering wheel.

- Road signs.

- Rear view mirror (center).

- Left mirror.

- Right mirror.

- In-vehicle other.

- Outside other.

In addition to categorizing the location of eye glances, the camera car system determined the length of glances to various locations. This ensured several methods for precise assessment of driver eye scanning behavior.

\section{Link Analysis of Driver Eye Scanning Behavior}

Link analysis was one method of analyzing eye-scanning behavior. The link analysis provided the probability of a glance to a given location and a glance transition between two different locations. When reviewing the link diagrams, it is important to remember that the values indicate the probability of a glance to a given location, not the proportion of glance time. The link diagrams for the six different navigation configurations are shown in figures 10 through 15 . The circles in the diagrams indicate the probability of a glance. Transition 
probabilities are provided adjacent to the lines between the circles. Note that to improve legibility, transition lines for proportions less than 0.01 are not provided.

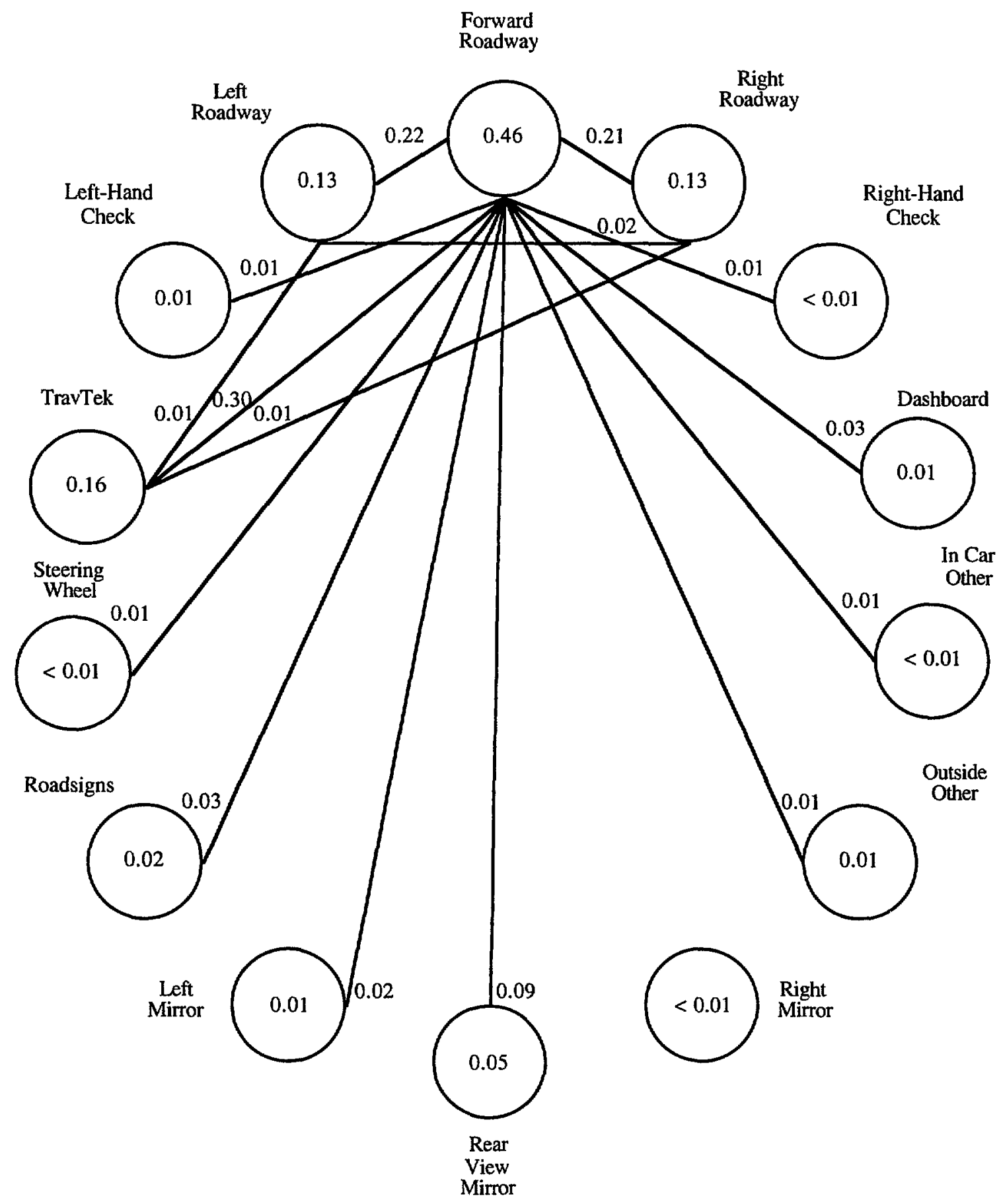

Figure 10. Glance probabilities for turn-by-turn with voice navigation condition. 


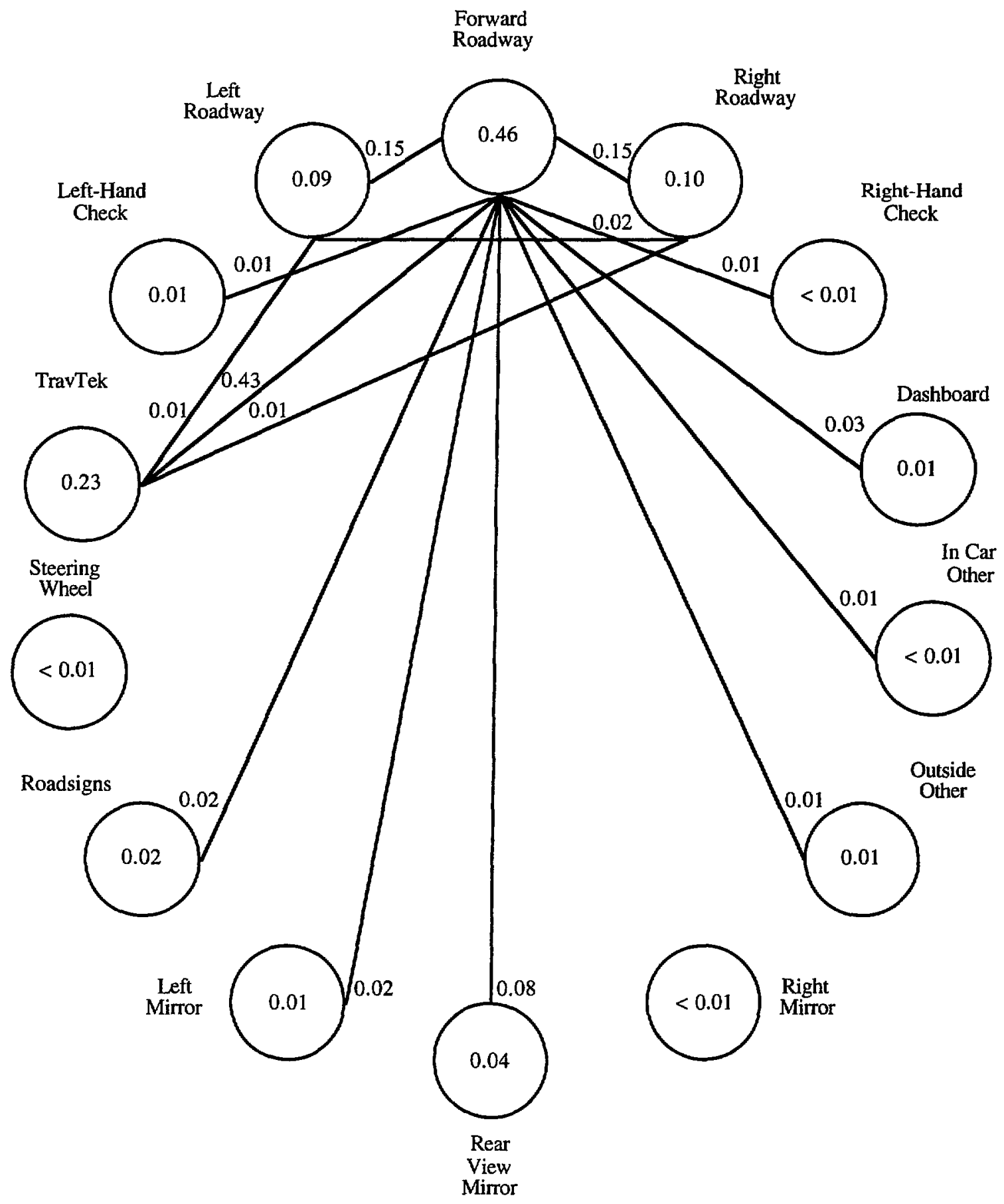

Figure 11. Glance.probabilities for turn-by-turn without voice navigation condition. 


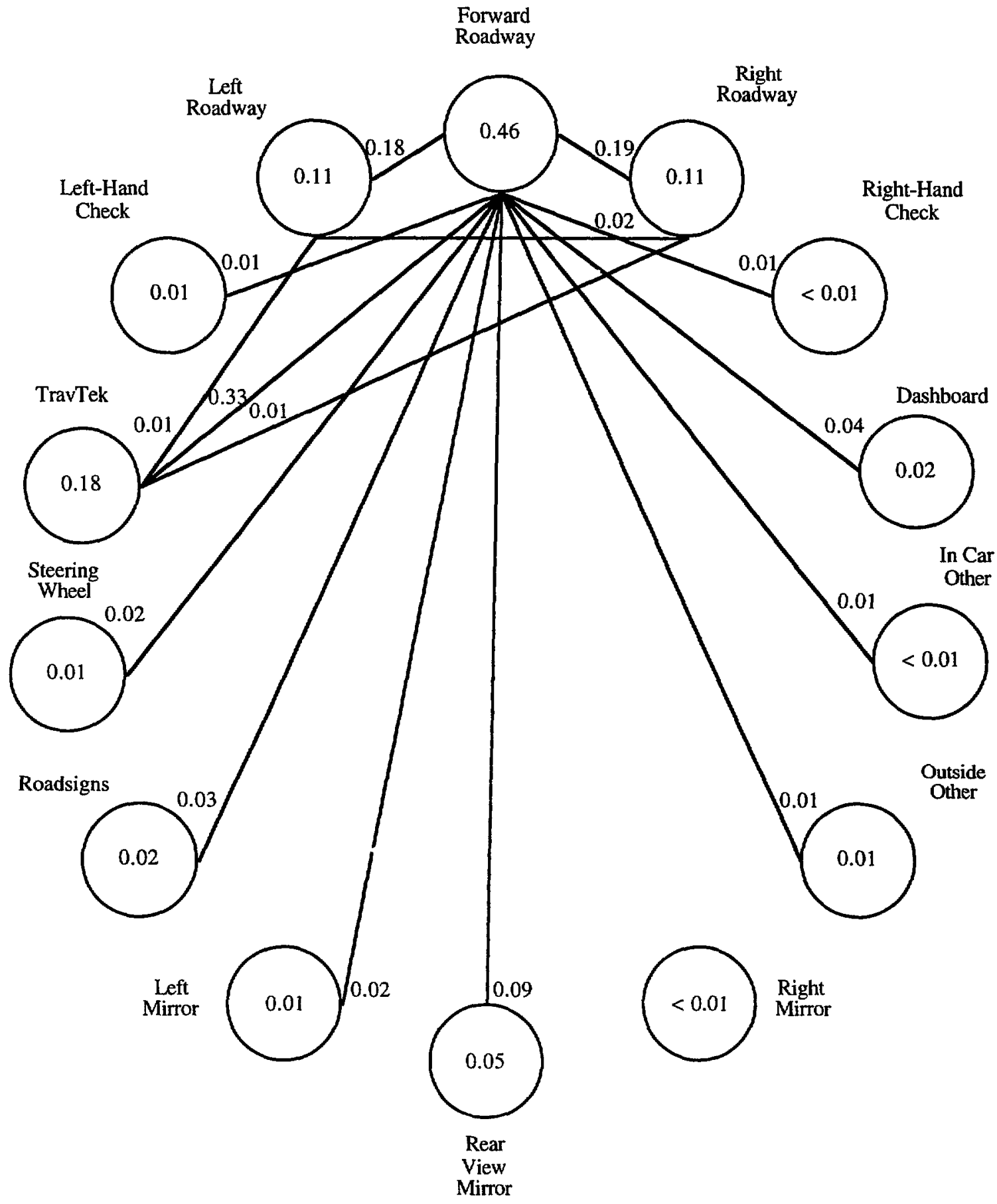

Figure 12. Glance probabilities for route map with voice navigation condition. 


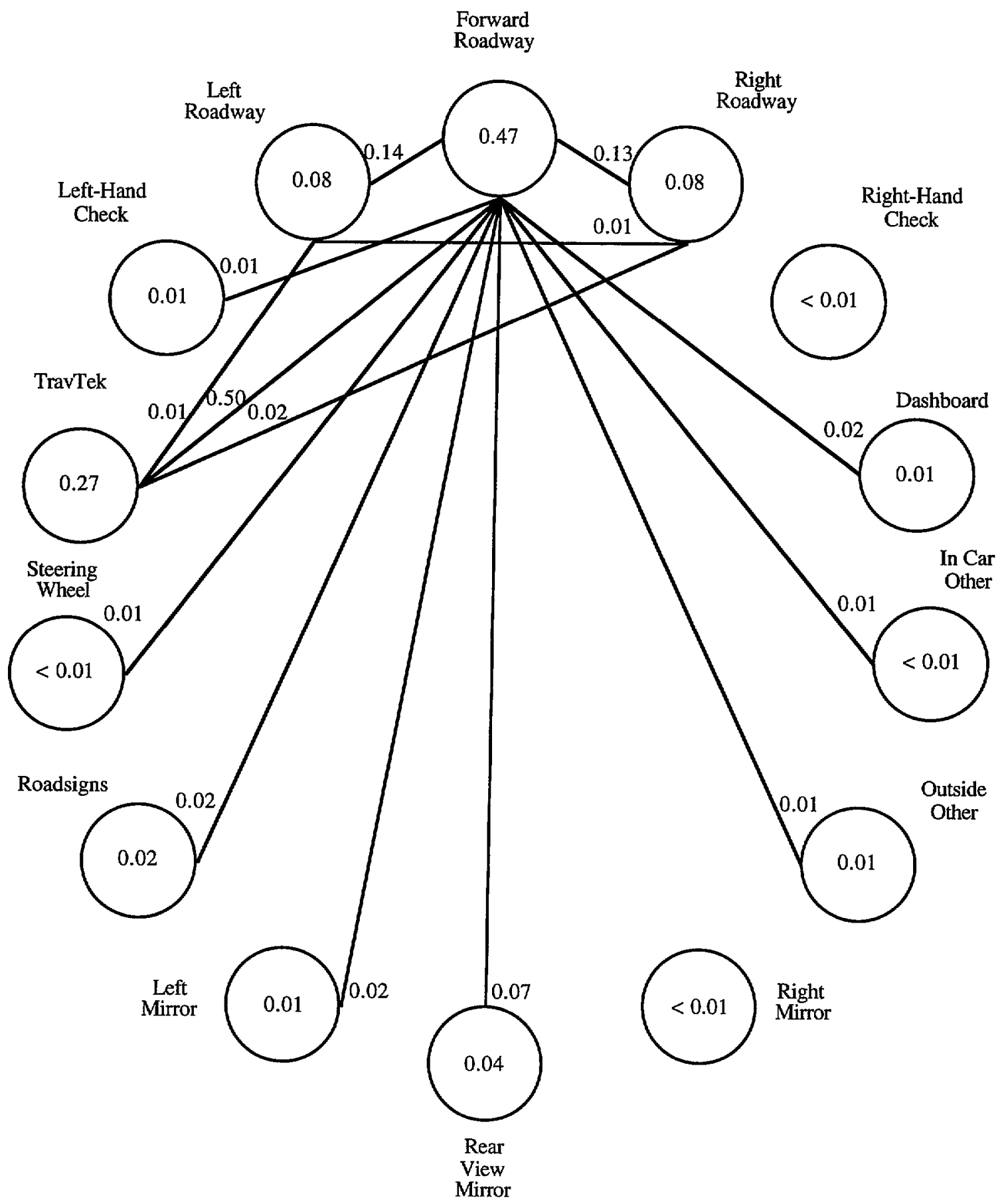

Figure 13. Glance probabilities for route map without voice navigation condition. 


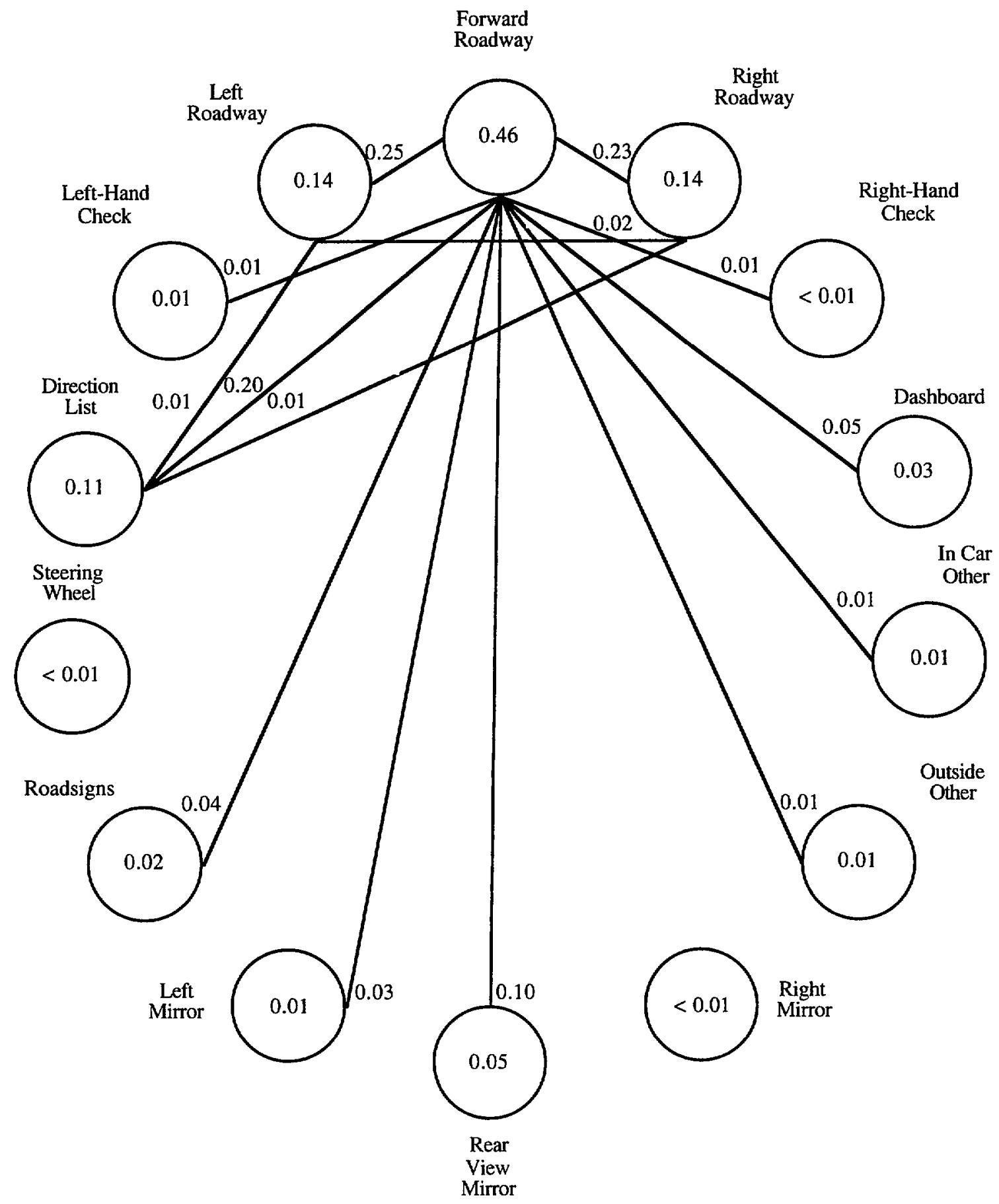

Figure 14. Glance probabilities for paper directions navigation condition. 


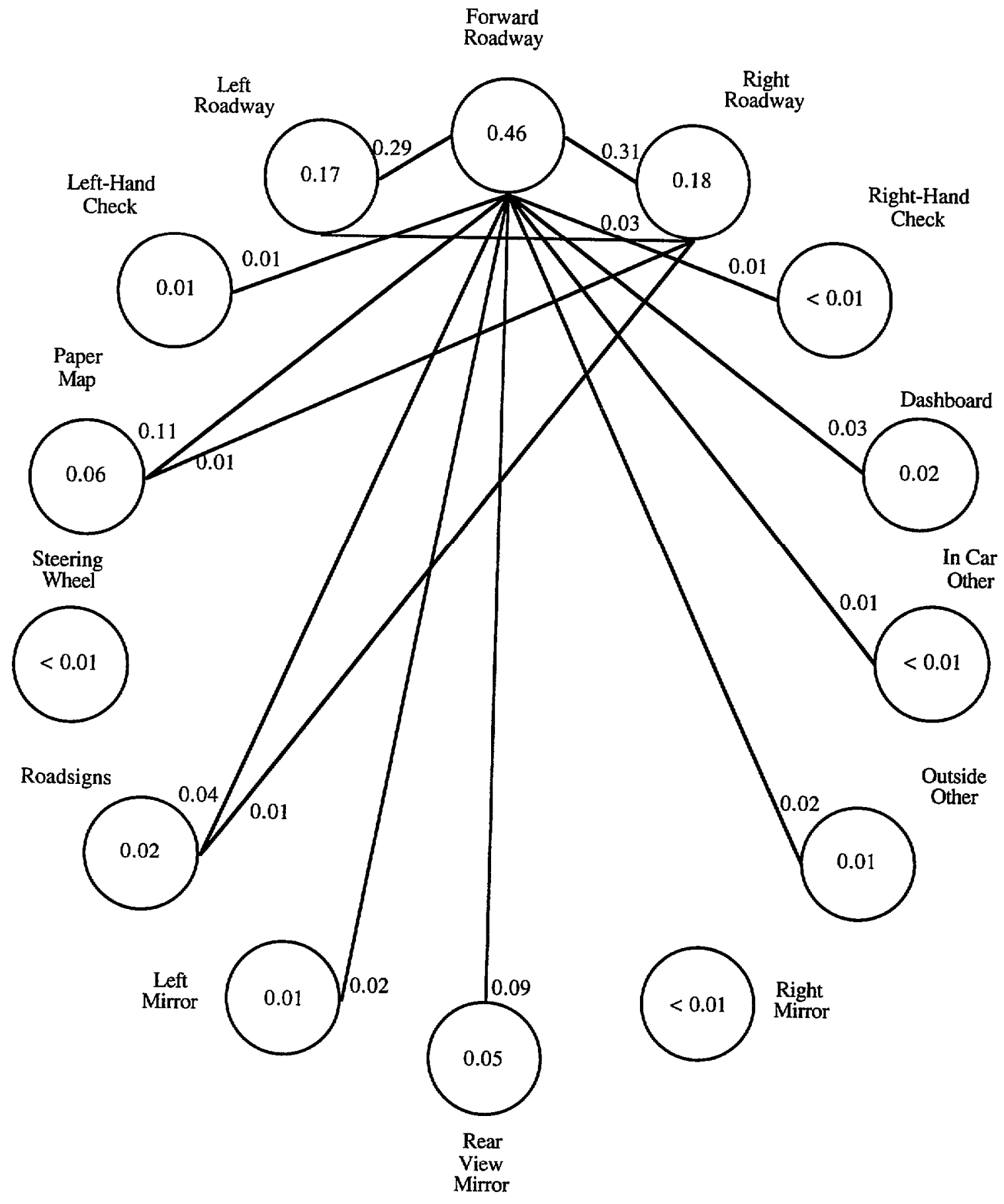

Figure 15. Glance probabilities for paper map navigation condition.

The link diagrams show that the percentage of glances to the forward roadway and rearview mirrors are remarkably consistent between the six navigation conditions. However, the percentage of glances to the left and right roadways, as well as the navigation
device, vary between conditions.

Figure 13 indicates that fewer samples were taken for the left and right roadway than other conditions. In contrast, figure 15 indicates that the paper map condition had the smallest 
proportion of navigation-related glances and the highest proportion of left and right roadway glances.

The six link diagrams also indicate that the presence of voice guidance is an apparent benefit and resulted in a reduction of glances to the TravTek display for the turn-by-turn and route map conditions by 7 percent and 9 percent respectively. As with the previous comparisons (see figures 11 and 13), these reductions also resulted in an increase in left and right roadway glances.

\section{Length and Number of Glances}

Figure 16 shows the total number of glances to each location for each display condition. This figure provides an overall perspective of glance locations, as well as major differences between navigation conditions. As shown, the forward roadway had the highest proportion of glances. The two TravTek conditions without voice had the most roadway glances, while the other four conditions were relatively equal. The two TravTek conditions without voice guidance also had the most display glances to the navigation condition. As discussed earlier in this section, the proportion of glances to the forward roadway for all TravTek navigation conditions was nearly equal. Because the total number of glances was higher while the proportions of glances to the forward roadway were the same as for other TravTek navigation conditions, it is apparent that there was more eye scanning activity when using the navigation conditions without voice. This activity was exhibited by a greater number of shorter glances between the roadway and the navigation device. The link analysis indicated that the paper map condition had the fewest navigation condition glances and the most left and right roadway glances.

The data from the measures above show that for configurations without voice, drivers make about twice as many glance transitions to the display as are made to configurations with voice. The increase in the number of transitions for configurations without voice result in a decrease in the average length of a glance to the forward roadway of about $0.5 \mathrm{~s}$. However, the average duration of glances to the display increases only by about $0.05 \mathrm{~s}$. Therefore, greatest contributing factor to driving intrusion between configurations with and without voice is the number of required transitions to the display. An increase in the number of transitions results in a reduction in the length of each glance to the forward roadway and the necessity to re-acquire traffic and other participants in the driving environment.

The results of ANOVA's performed to test differences in glance length to the navigation aids and "roadway-related" features for the navigation conditions are presented in table 7.

Significant differences were found for duration of glances to navigation aids and duration of roadway related glances. Roadway-related glances were operationally defined to include the forward roadway, left roadway, right roadway, left-hand check, right-hand check, and mirror locations. This operational definition was created to account for all glances being performed to seek information from the roadway environment. Navigation glances are defined as an eye fixation directed on the navigation device being tested (TravTek display, paper map, or paper direction list).

The mean duration of glances to different navigational aid locations is shown in figure 17. As supported by previous literature, the graph shows that glances away from the forward roadway were of an average duration of 1.0 Splus or minus 0.3 S(22) Figure 17 indicates, that with the exception of the forward roadway, there were no large differences in glance durations among the navigation conditions. Note that the two TravTek conditions without 


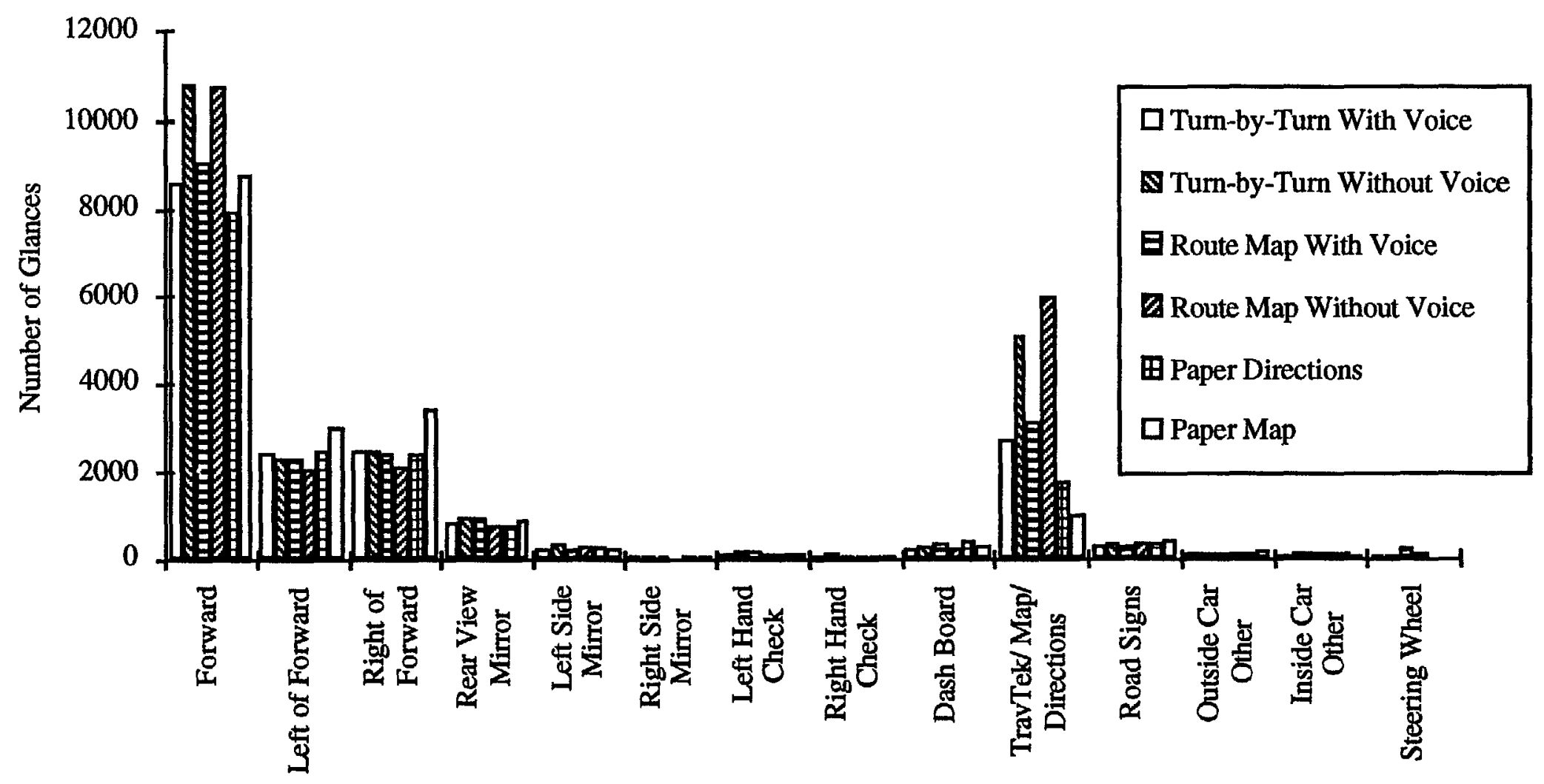

Figure 16. Number of glances to each glance location and each navigation condition; all possible locations included. 
voice apparently had the shortest average glance durations to the forward roadway. These conditions had a larger number of shorter glances to the roadway compared to the other navigation conditions.

Table 7. ANOVA's testing differences in glance length to the navigation aids and roadwayrelated features.

\begin{tabular}{|c|c|c|c|c|}
\hline \multicolumn{5}{|c|}{ Duration of Glances to Navigation Aid } \\
\hline Source & Df & MS & $\mathrm{F}$ & $\mathrm{P}$ \\
\hline Navigation condition & 5 & 28.3222 & 10.81 & 0.0001 \\
\hline Navigation condition X Subjects & 136 & 2.6211 & & \\
\hline
\end{tabular}

\begin{tabular}{|c|c|c|c|c|}
\hline \multicolumn{5}{|c|}{ Duration of Roadway Related Glances } \\
\hline Source & Df & MS & $\mathrm{F}$ & $\mathrm{P}$ \\
\hline Navigation condition & 5 & 376.0366 & 15.85 & 0.0001 \\
\hline Navigation condition X Subjects & 136 & 23.7252 & & \\
\hline
\end{tabular}

Graphs showing SMM post-hoc statistical comparisons between navigation conditions for duration of roadway-related and navigation glances appear in figures 18 and 19. Figure 18 indicates that the without voice condtions had significantly shorter roadway glance times than turn-by-turn with voice, paper direction and paper map. The turn-by-turn with voice, paper direction list, and paper map conditions had the longest roadway glance times.

Figure 19 shows that the turn-by-turn with voice condition had significantly shorter navigation glance times than the route map without voice, paper directions and paper map conditions. All other conditions were not statistically different.

Figure 20 shows a breakdown of the number of glances to navigation locations by glance length. Driving task intrusion is particularly evident by large numbers of long display glances. As shown, the majority of glances were under $2.0 \mathrm{~s}$. Previous researchers have used $2.5 \mathrm{~s}$ as a criterion for an unsafe glance length. Although most of the glances were less than $2 \mathrm{~s}$, the route map without voice condition had over 300 glances between 2.0 and $3.0 \mathrm{~s}$ across 42 experimental drives. Although the turn-by-turn with voice had a large number of glances, many of those glances were under $1 \mathrm{~s}$. The paper map condition had the fewest glances overall, and the turn-byturn with voice and paper map conditions had the fewest glances over $2 \mathrm{~s}$.

A further analysis of the number of glances between 2.0 and $3.0 \mathrm{~S}$ indicates a trend for both the use of voice and the information presentation format for the TravTek navigation conditions. When comparing turn-by-turn with voice to turn-by-turn without voice or route map with voice to route map without voice, fewer glances were made in longer length categories when voice was included. This means that the incorporation voice resulted in fewer navigation glances of long duration in addition to just a larger number of glances overall. This trend shows a potential safety benefit with the inclusion of voice guidance. A similar trend can be seen for the format of information presentation. Comparing the number of glances for route map with voice to turn-byturn with voice the route map condition required more glances. A comparison of the number of glances for route map without voice to turn-by-turn without voice, reveals that the route map condition resulted in additional longer glances. The route map consistently required more glances over $1 \mathrm{~s}$ than the turn-by-turn format. This effect can be seen in figure 20. As with the inclusion of voice, the simpler turn-by-turn format has a potential safety benefit in that it does not require as many long glances to the display. 


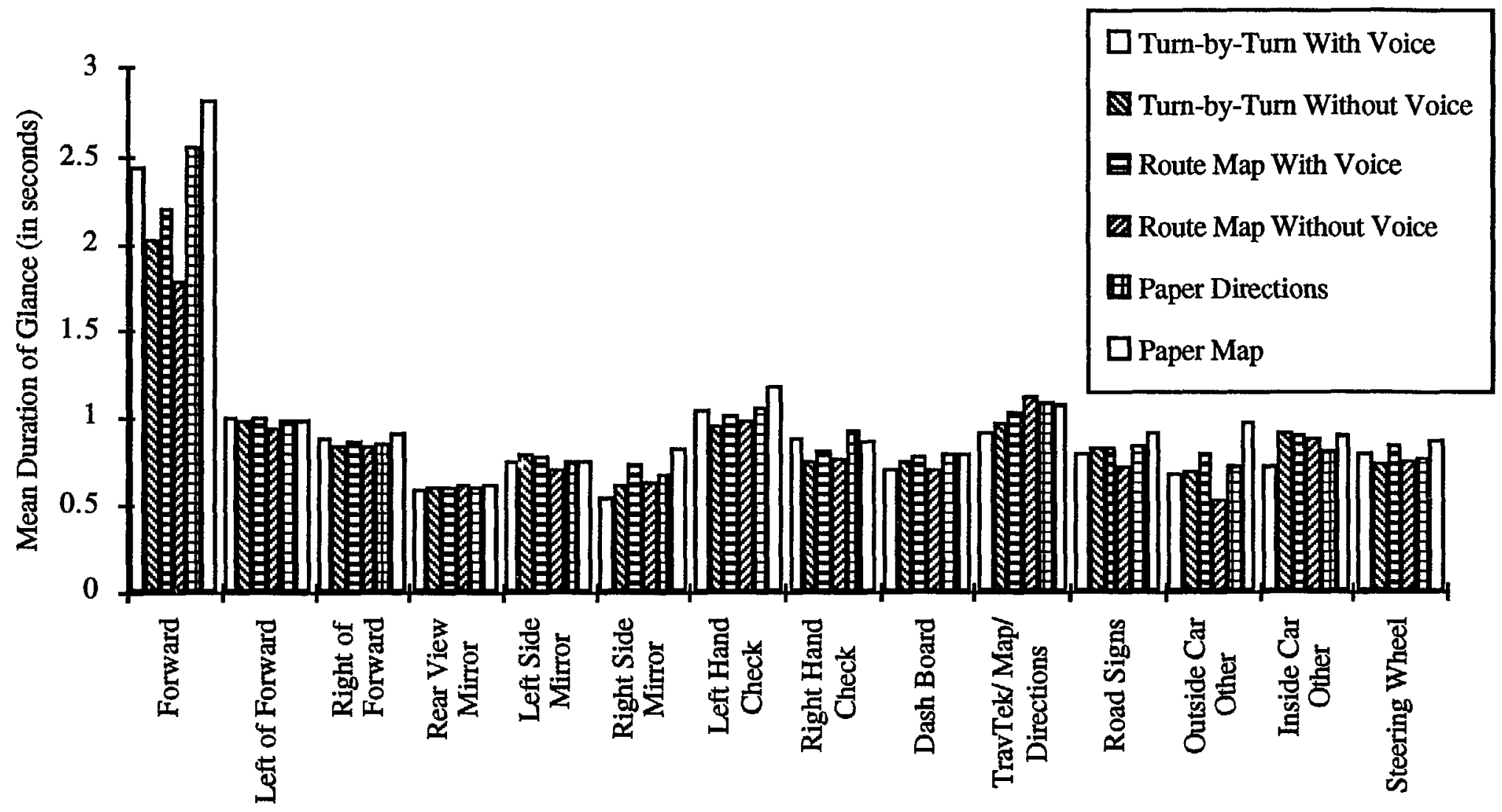

Figure 17. Duration of glances for each navigation condition; all glance locations included. 


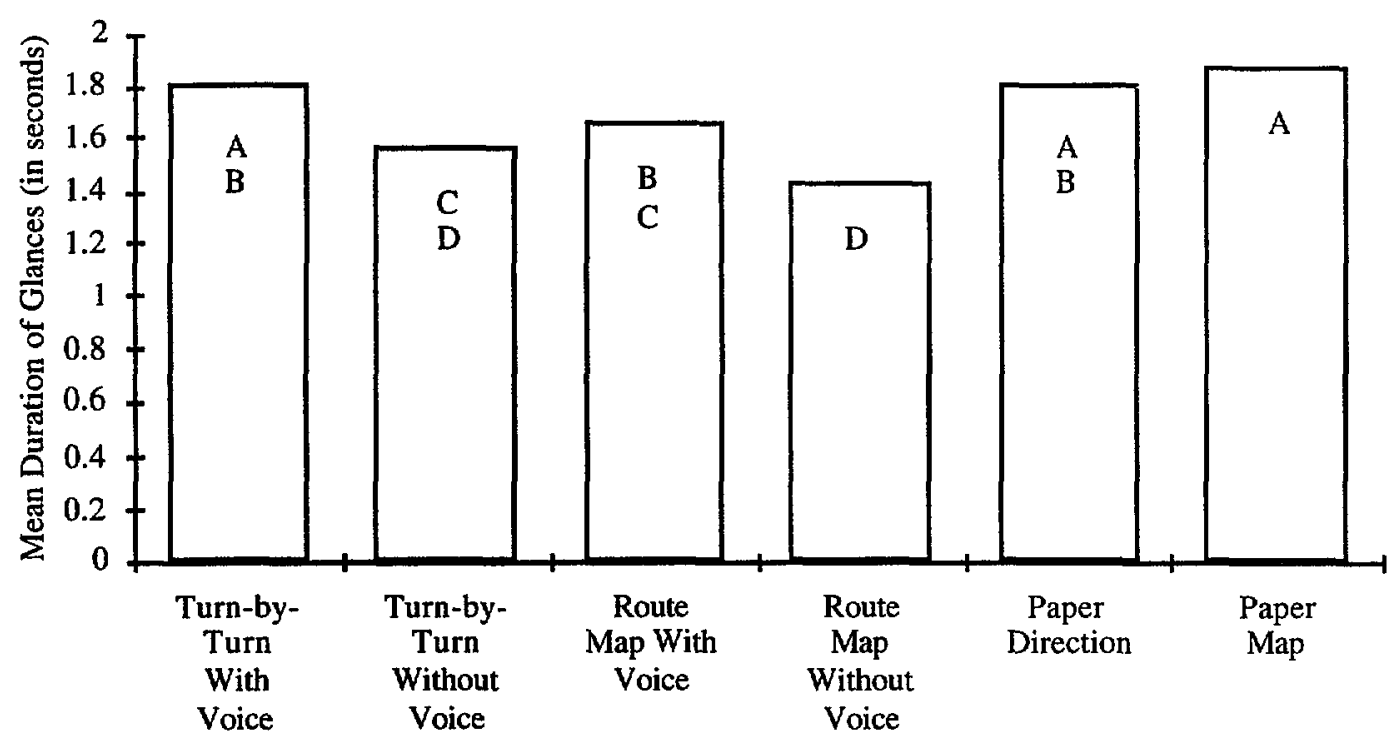

Figure 18. Duration of glances for each navigation condition; only glances to the roadway included

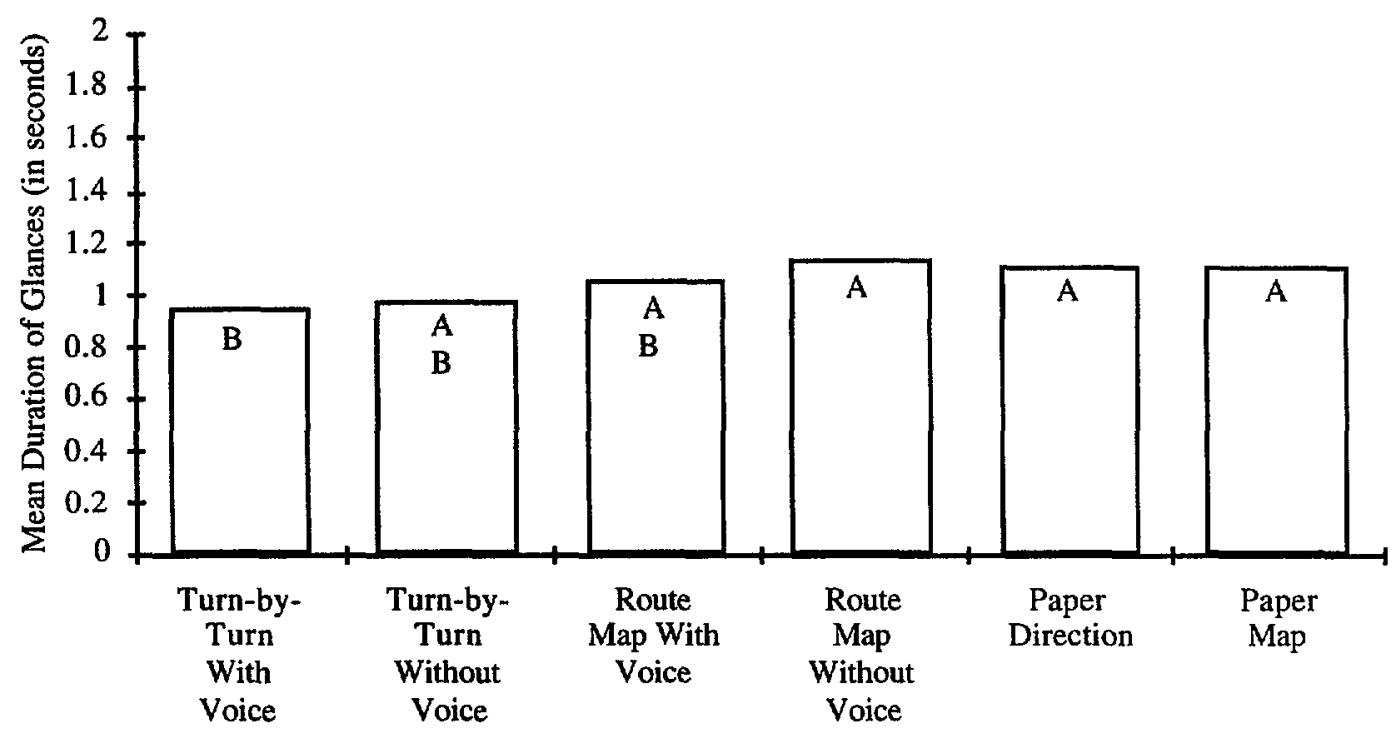

Figure 19. Duration of glances for each navigation condition; only glances to navigation display included.

One possible explanation for lower number of glances to the paper map is that drivers took time to study and memorize the map as they were planning their route and creating directions to follow. Results in the next section show that drivers took significantly longer to plan a route using the paper map than any other condition. Since they may have had a better mental representation of the route, they required fewer glances to the paper map to navigate to their destination. In addition, results in the next section show that drivers stopped more often in the paper map condition. That is, drivers apparently studied the 
paper map while stopped as opposed to while in-motion. This would help explain the differences in numbers of glances. Drivers probably relied more on the TravTek displays for incremental pieces of information during their experimental drives rather than navigating from a mental representation of the route in memory, and stopped as needed along the route to refresh that memory.

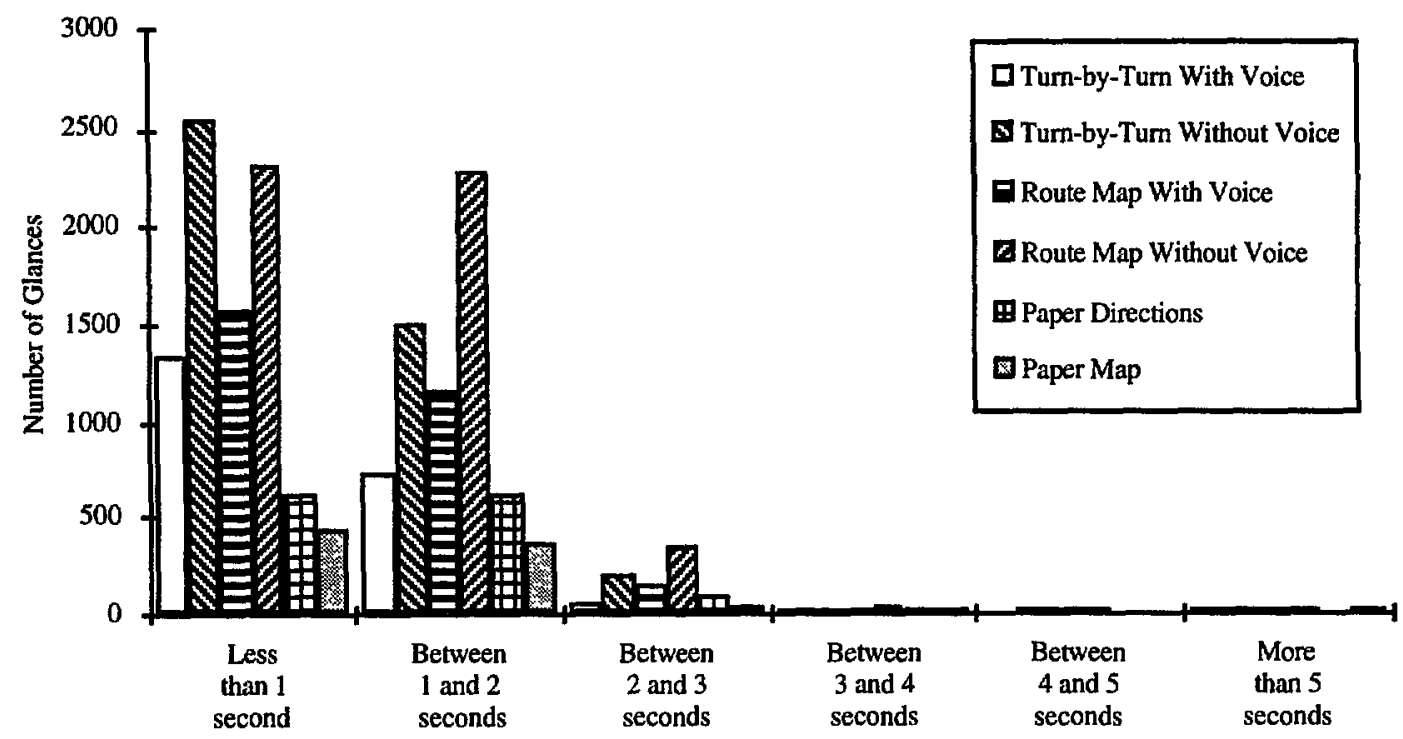

Figure 20. Number of glances to the navigation display shown by varying glance durations.

Eye glance behavior in intersections is an important driving task intrusion issue. Intersections require a heightened degree of awareness in order to drive safely due to the increased number of potential accident types and threat locations. Intersections also serve as landmark points of reference to the navigating driver. Therefore, eye scanning behavior while in intersections is particularly important. An intersection analysis for the eye glance data was conducted and divided into two categories. The first category (referred to as intersections), included those instances when the camera car driver was in an intersection, but was not making a turn. Since the data were reduced by each eye glance, these data include any glance which occurred while in an intersection. The second category (referred to as "turns"), included those instances where the driver was required to turn in an intersection.

The number of glances to various intersection category locations is shown in figure 21 . The largest number of glances overall, were to the forward roadway, with left and right roadway and navigation condition having the only other substantive number of roadwayrelated glances. The paper map condition had the largest number of roadway glances and the fewest number of navigation glances. The two TravTek conditions without voice had the highest number of navigation glances while in intersections. The turn-by-turn with voice and paper direction list conditions had relatively low numbers of display glances.

Although the differences were small and may not be statistically significant, the paper map condition had the largest number of road sign glances. 


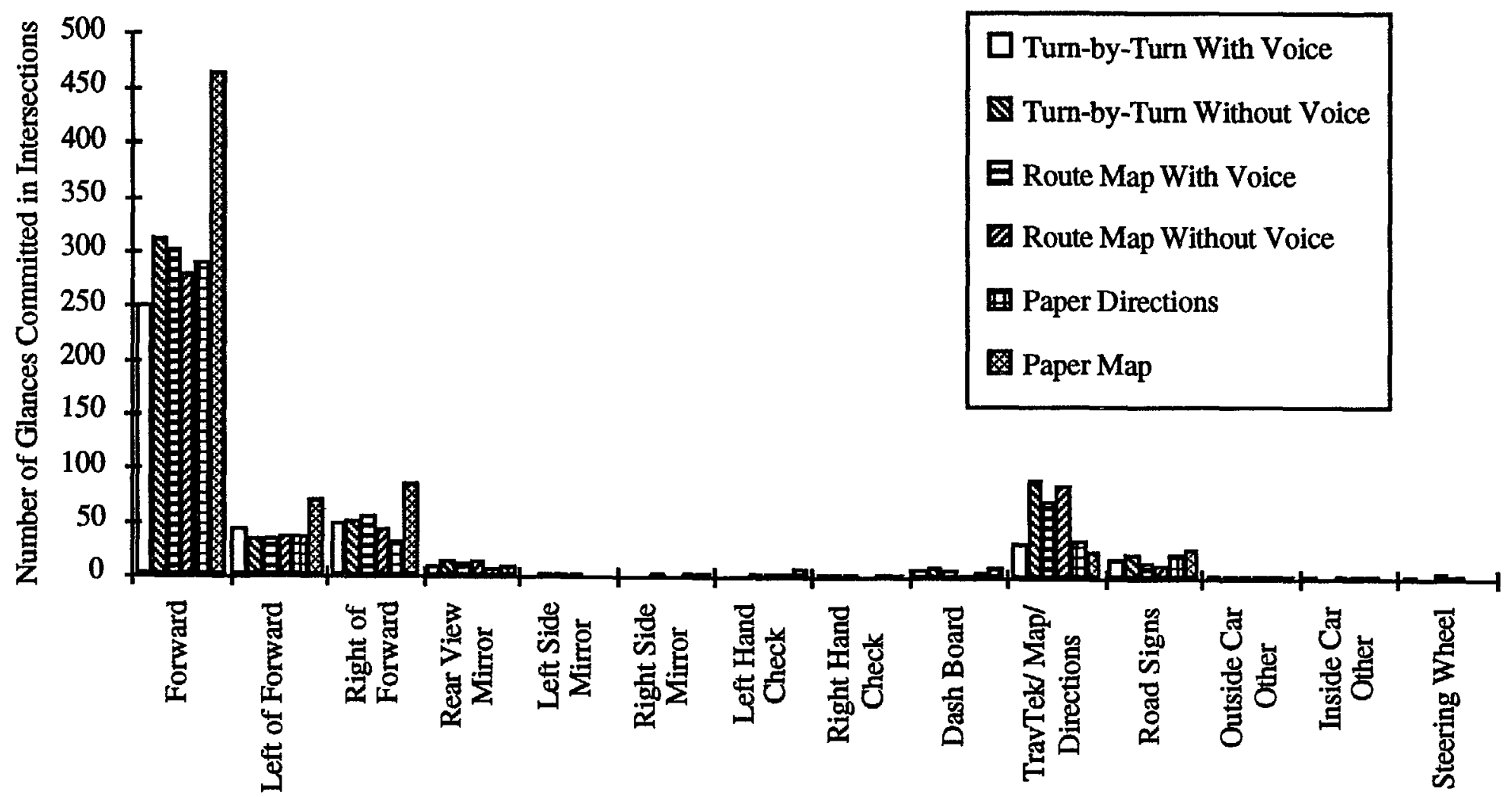

Figure 21. Number of glances for each glance location and each navigation condition; only glances occurring in intersections included. 
Long driver glances to the navigation condition while in intersections constituted a face-valid measure of driving task intrusion. Figure 22 shows the number of display glances over $2 \mathrm{~s}$ while in intersections. Although the number of glances overall is low, the route map without voice condition had more than twice as many (11 as opposed to 5) as the next highest condition.

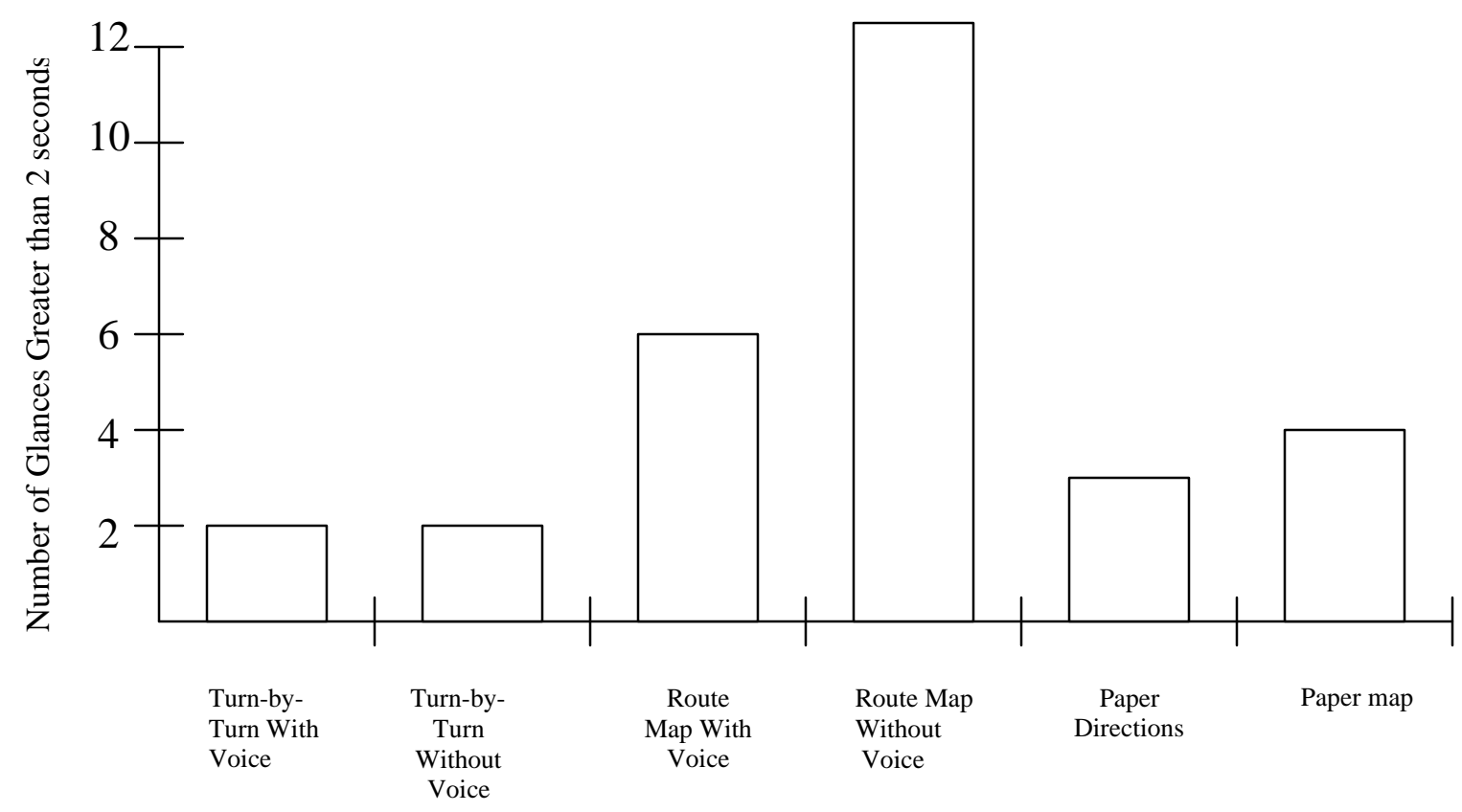

Figure 22. Number of glances tor each navigation condition; to the navigation display lasting over $2 \mathrm{~s}$ that occurred in intersections.

The number of glances to various locations during turns appears in figure 23. Note that glances were only recorded and analyzed in the Camera Car Study when the vehicle was in motion. In general, there is a much higher proportion of glances to the left and right roadway in turns than in the intersection analysis. The route map with voice had the highest number of roadway glances. The two TravTek conditions without voice had the highest number of navigation glances; however, the paper direction list condition was also high. The paper direction list condition had low left and right roadway glances and no rear view mirror glances. These results are somewhat contradictory to the previous findings, but the strategy for using the paper direction list may include an occasional verification check during the turn to ensure that the turn street is indeed correct. This may also be the case for the TravTek conditions without voice.

As with the intersection data, an analysis was run to establish the number of instances where subjects had long display glances during turns. This analysis showed that only six glances were present for all six navigation conditions. This result indicated that drivers rarely glanced for long periods of time under such circumstances, and that any differences are not reliable. Therefore, we can conclude that drivers typically do not make long glances to any navigation aid during turns. 


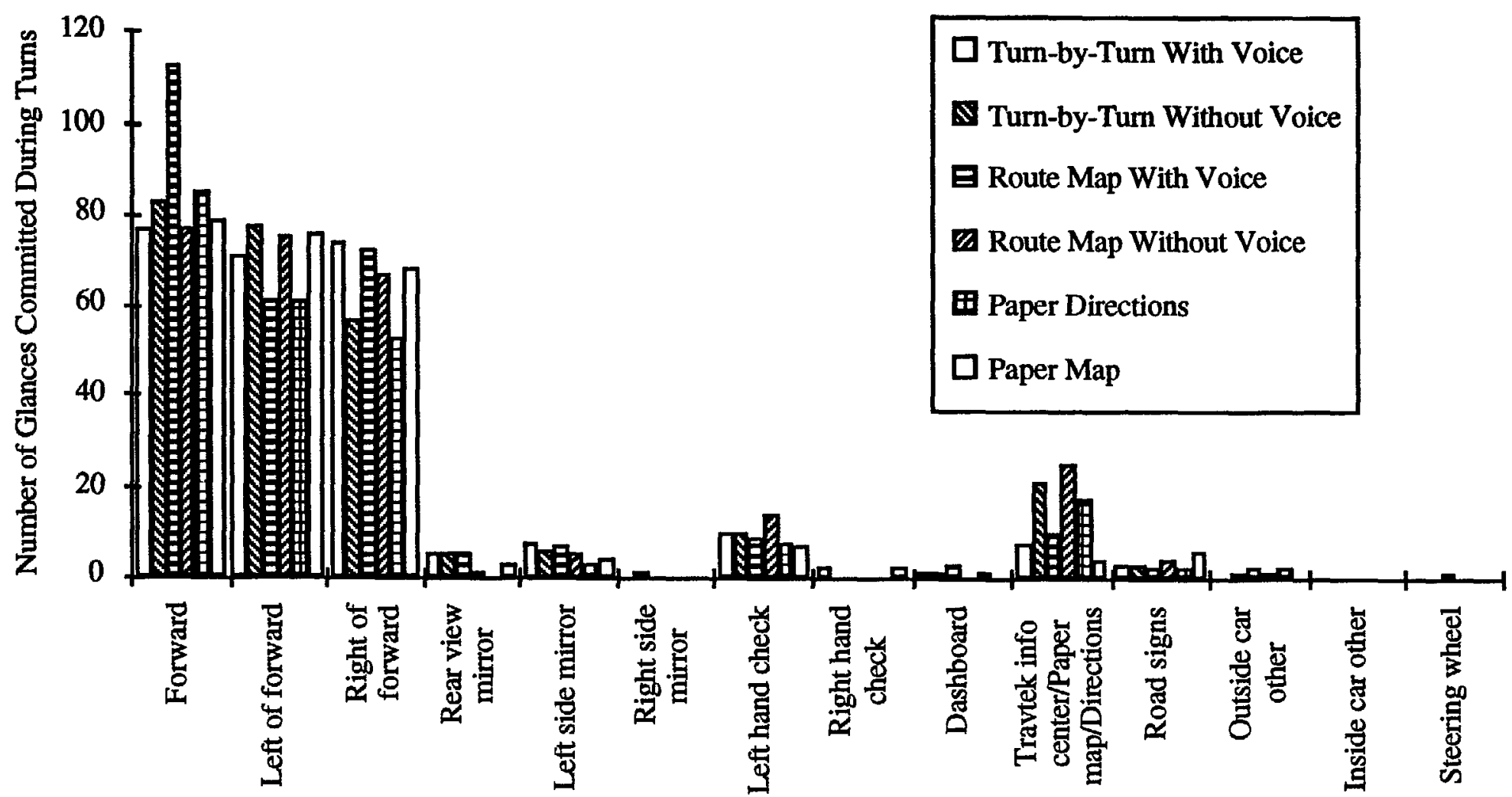

Figure 23. Number of glances for each glance location and each navigation condition; only glances occurring during turns included. 


\section{Measures of Driving Performance}

ANOVA's were performed for a variety of measures indicative of driving performance. The ANOVA's for these variables are shown in table 8. The dependent variables (i.e., average number of abrupt lateral maneuvers, average number of brake applications, average number of steering reversals, steering wheel position variance, average negative longitudinal acceleration, and variance in negative longitudinal acceleration), did not show any significant differences among navigation conditions at the $p<0.05$ level. The results of post-hoc tests utilizing the SMM statistic are described below for each of the remaining variables.(24)

\section{Number and D uration of Lane D eviations}

The number of lane deviations for each navigation condition was relatively uniform across display types (figure 24). These measures were analyzed as an indication of the safety impacts of using the system. The TravTek turn-by-turn conditions had the lowest number of lane deviations. The other four conditions had a somewhat higher and relatively uniform number of lane deviations. Although no statistical tests were conducted, these results indicate that there is a safety benefit, as least for novice visitors and local users, for the TravTek turn-by-turn configurations.

\section{Lateral Acceleration Variance}

Although the ANOVA indicated a statistical difference for variance in lateral acceleration, the SMM post-hoc test indicated that no differences were present. The SMM was selected because it is a conservative post-hoc test. This may be the reason for the inconsistency. The order of the means from lowest to highest was the turn-by-turn with voice condition, followed by turn-by-turn without voice, route map with voice, paper directions, route map without voice, and paper map. Note that this order does not represent statistical differences, and is presented here only as trend information.

\section{VehicleVelocity}

Figures 25 and 26 show the means and SMM results for speed and speed variance respectively. The mean speed for the paper map condition was less than the other five conditions. The remaining navigation conditions were not statistically different from one another. As discussed in the issues section of this report, previous research has indicated that slower average speed indicates higher driver task load. The paper map condition had a smaller speed variance than the direction list, turn-by-turn without voice, and route map without voice conditions (see figure 26). In general, an increase in speed variance indicates poorer driving performance. However, the paper map condition may have had the lowest variance in speed due to the lower average speed driven. It may also be the case that when using the paper map, drivers were memorizing the route to some extent and using their representations in memory rather than always referring to the map or to the directions they created. The resulting reduction in visual attention could have positively influenced velocity variance. 
Table 8. ANOVA's for driving performance.

Variance in Lateral Acceleration

Source

Navigation condition

Navigation condition X Subjects

\begin{tabular}{|r|l|l|l|}
\hline \multicolumn{1}{|c|}{$\mathrm{dF}$} & $\mathrm{MS}$ & $\mathrm{F}$ & $\mathrm{P}$ \\
\hline & 5 & 0.000002 & \multicolumn{2}{|c|}{2.93} & 0.0150 \\
\hline & 139 & 0.000007 & \multicolumn{3}{|c|}{} \\
\hline
\end{tabular}

Variance in Steering Position

Source

Navigation condition

Navigation condition X Subjects

Variance in Longitudinal Acceleration

Source

Navigation condition

Navigation condition X Subjects

Mean Speed

Source

Navigation condition

Navigation condition X Subjects

\begin{tabular}{|c|c|c|c|}
\hline $\mathrm{dF}$ & MS & $\mathrm{F}$ & $\mathrm{P}$ \\
\hline 5 & 105936.038 & 0.01 & 0.4158 \\
\hline 139 & 105157.4 & & \\
\hline $\mathrm{dF}$ & MS & $\mathrm{F}$ & $\mathrm{P}$ \\
\hline 5 & 0.0000044 & 2.80 & 0.193 \\
\hline 139 & 0.0000016 & & \\
\hline
\end{tabular}

\section{Speed Variance}

Source

Navigation condition

Navigation condition X Subjects

\begin{tabular}{r|r|r|r|}
\multicolumn{1}{r|}{$\mathrm{dF}$} & $\mathrm{MS}$ & $\mathrm{F}$ & $\mathrm{P}$ \\
\hline 5 & 55.2937 & 9.17 & 0.0001 \\
\hline 139 & 6.0326 & \multicolumn{3}{|c|}{} \\
\hline
\end{tabular}

Negative Longitudinal Acceleration

\begin{tabular}{|l|r|r|r|r|}
\hline Source & dF & MS & \multicolumn{1}{|c|}{ F } & P \\
\hline Navigation condition & 5 & 0.000026 & 0.64 & 0.6691 \\
\hline Navigation condition X Subjects & 139 & 0.000041 & & \\
\hline
\end{tabular}

Variance in Negative Longitudinal Acceleration

Source

Navigation condition

Navigation condition X Subjects

\begin{tabular}{r|r|r|r|}
\multicolumn{1}{r|}{$\mathrm{dF}$} & \multicolumn{1}{|c|}{ MS } & $\mathrm{F}$ & $\mathrm{P}$ \\
\hline 5 & 6269.3600 & 3.52 & 0.0005 \\
\hline 139 & 1780.54 & \multicolumn{3}{|c|}{} \\
\hline
\end{tabular}

Number of Steering Reversals Correct by Travel Time

Source

Navigation condition

Navigation condition X Subjects

\begin{tabular}{r|c|c|c|}
\multicolumn{1}{|l|}{$\mathrm{dF}$} & $\mathrm{MS}$ & $\mathrm{F}$ & $\mathrm{P}$ \\
\hline 5 & 0.0000008 & 1.16 & 0.3325 \\
\hline 139 & 0.0000007 & \multicolumn{3}{|c|}{} \\
\hline
\end{tabular}

Number of Brake Applications Corrected by Travel Time

\begin{tabular}{|c|c|c|c|c|}
\hline Source & $\mathrm{dF}$ & MS & $\mathrm{F}$ & $\mathrm{P}$ \\
\hline Navigation condition & 5 & 0.0012 & 1.82 & 0.1130 \\
\hline Navigation condition X Subjects & 136 & 0.0005 & & \\
\hline \multicolumn{5}{|c|}{ Time for Which the Brake is Pressed } \\
\hline Source & $\mathrm{dF}$ & MS & $\mathrm{F}$ & $\mathrm{P}$ \\
\hline Navigation condition & 5 & 27302.57 & 9.86 & 0.0001 \\
\hline Navigation condition $X$ Subjects & 139 & 2768.74 & & \\
\hline
\end{tabular}




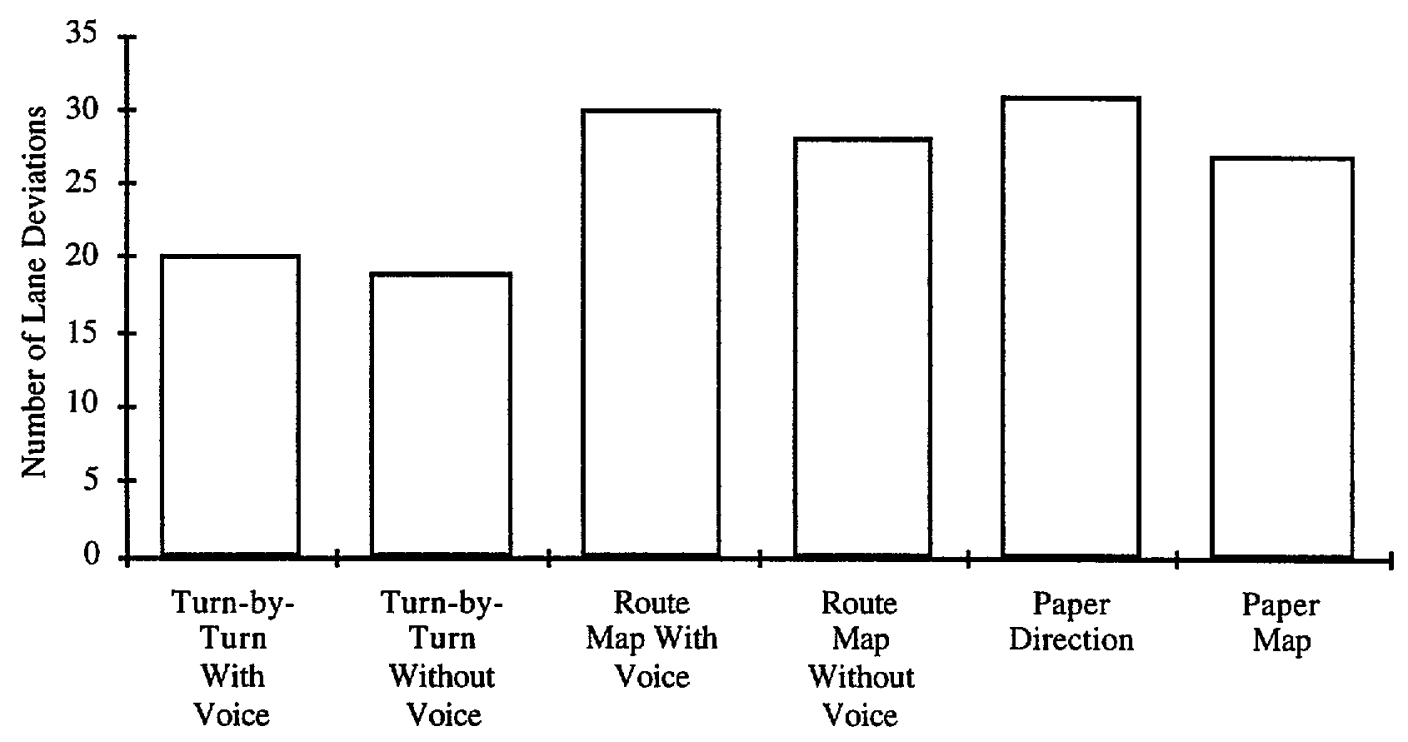

Figure 24. Number of lane deviations for each navigation condition.

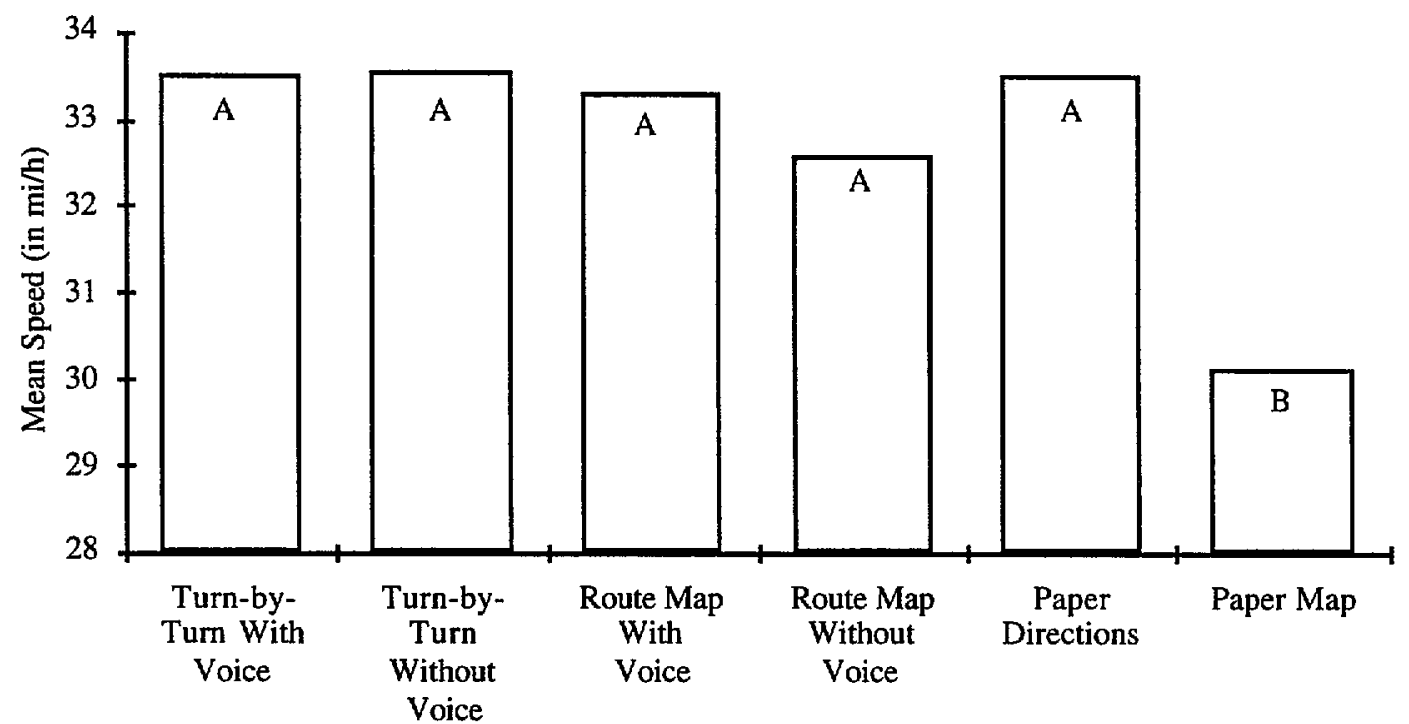

Figure 25. Mean speed for each navigation condition. $(1 \mathrm{mi} / \mathrm{h}=1.61 \mathrm{~km} / \mathrm{h})$

\section{Longitudinal Acceleration Variance}

As with the variance in lateral acceleration, variance in longitudinal acceleration was statistically significant for the navigation conditions in the ANOVA (see table 8).

However, the SMM revealed no significant difference between display type. Again, this finding is probably due to the conservative post-hoc test. 


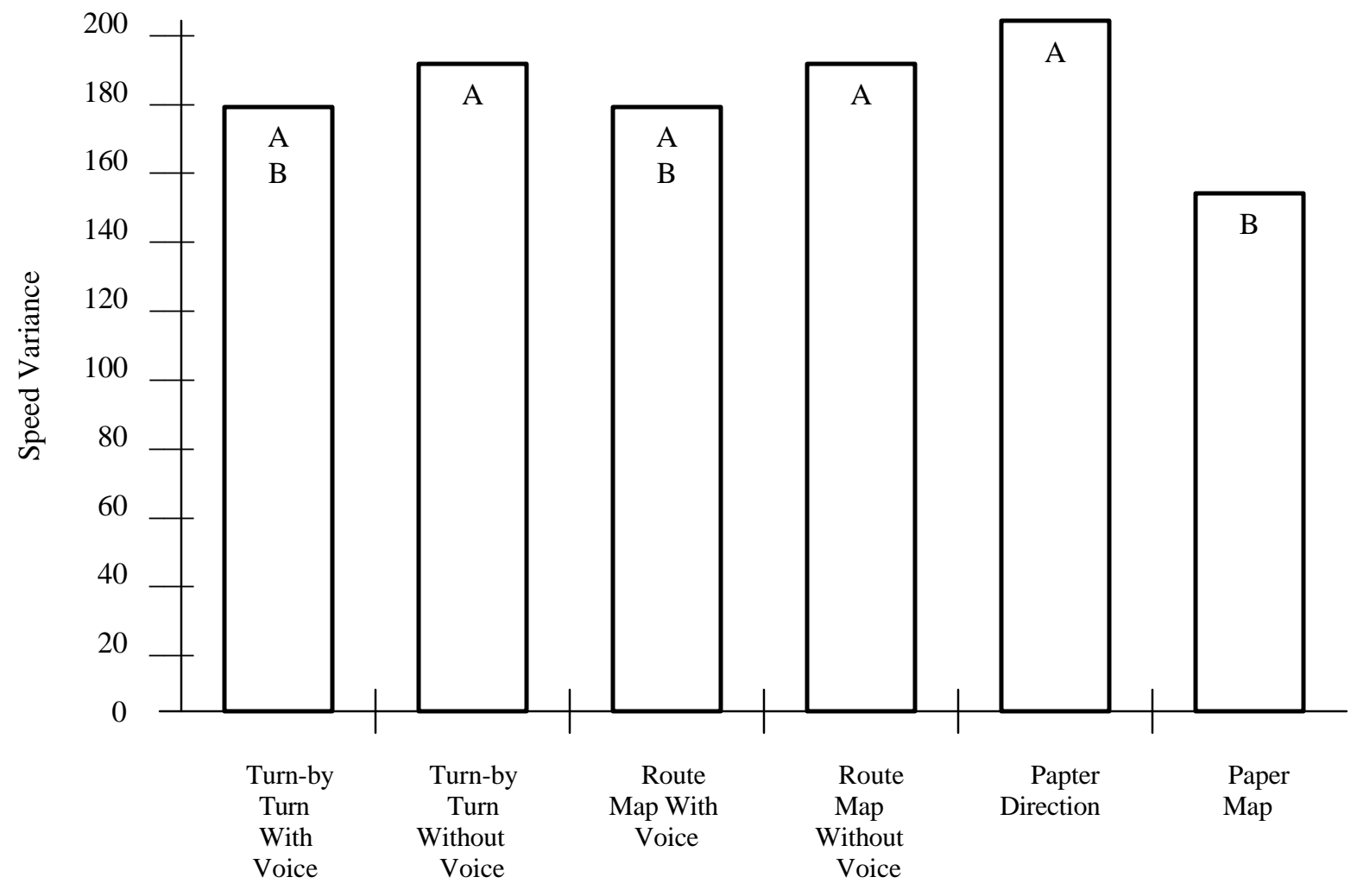

Figure 26. Speed variance for each navigation condition.

\section{Abrupt Braking Maneuver}

The negative longitudinal acceleration and variance in negative longitudinal acceleration were not significant (see table 8). However, the average number of abrupt braking maneuvers were different although no statistical test was run. An "abrupt maneuver" in this instance was operationally defined as exceeding the criterion of the first percentile negative longitudinal acceleration value for all of the data. The number of maneuvers for each display type is shown in figure 27 . The turn-by-turn with voice and turn-by-turn without voice conditions had lower average numbers of abrupt maneuvers than the paper map condition. In general, abrupt maneuvers can be indicative of a lack of driver attention on the driving task. This result is somewhat counter-intuitive, however, given that paper map runs were the slowest and that the paper map required the fewest of navigation glances. This result likely reflects the greater number of stops present in the paper map condition discussed in the next section. That is, since drivers were required to stop more to look at the map, they performed more abrupt maneuvers while making those stops. This is consistent with the observations of the in-vehicle experimenter, who noted that drivers using the paper map often slowed to turn into a parking lot to study the map.

\section{Analysis of Driver Mental Workload}

As previously described in the methods section, drivers were asked to provide a subjective workload rating at several points along each drive. The subjective workload rating scale is a three-dimensional scale which provides a subjective measure of the time stress, psychological stress, and mental effort load that the subject experienced. Each dimension of the subjective workload scale receives a rating of 1 (low), 2 (moderate), or 3 (high). Each one of these dimensions is reported below across navigation conditions, as well as a sum of the individual scale ratings. (For a more detailed description of this rating system, see the TravTekYoked Driver Study - Cl Final Report.) The ANOVA's for the overall subjective workload rating and the individual dimensions appear in table 9. 


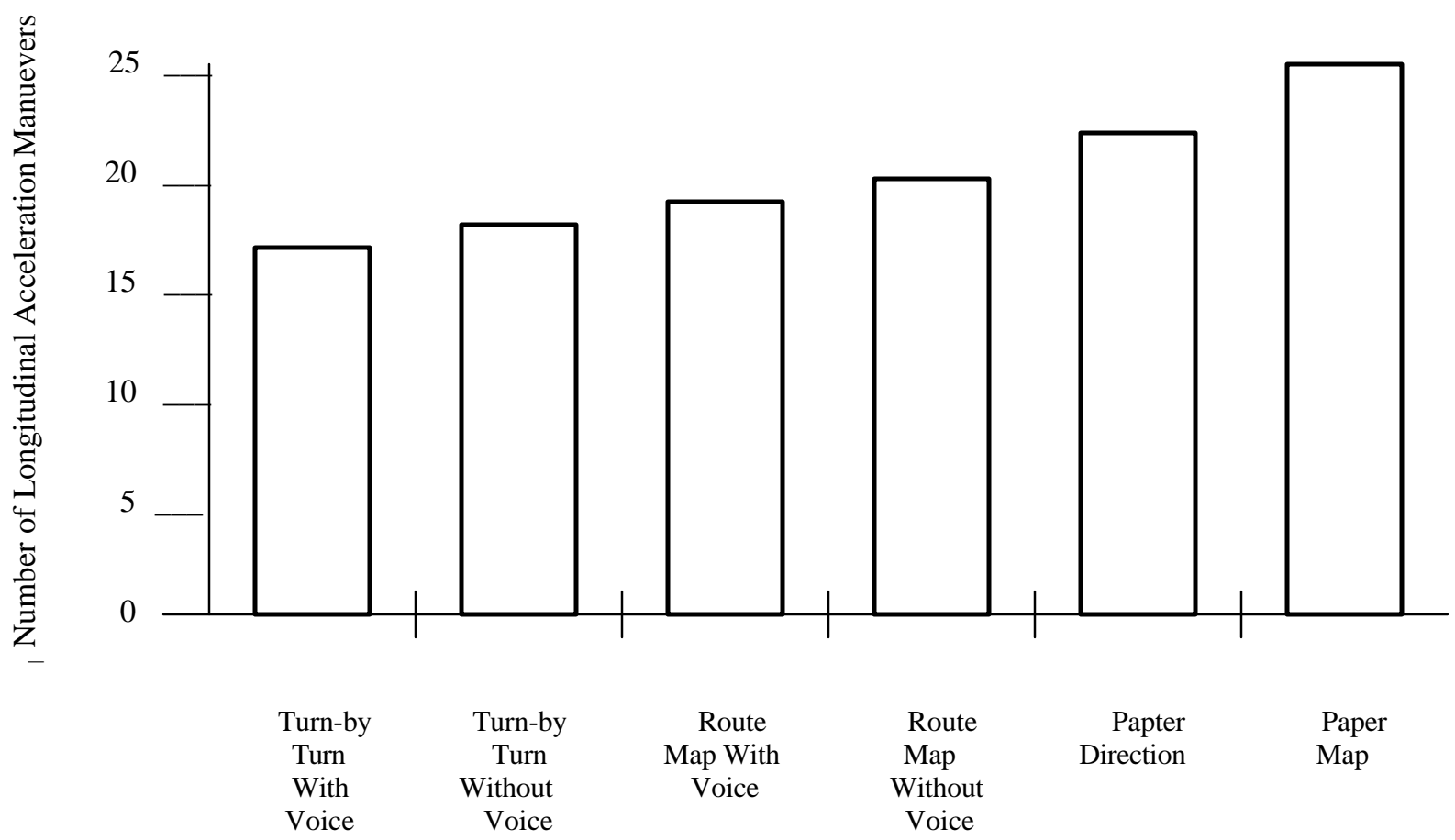

Figure 27. Number of abrupt longitudinal acceleration maneuvers for each navigation condition.

Table 9. ANOVA's for workload ratings.

\begin{tabular}{|c|c|c|c|c|}
\hline \multicolumn{5}{|l|}{ Overall workload Ratings } \\
\hline Source & $\mathrm{dF}$ & MS & $\mathrm{F}$ & $\mathrm{P}$ \\
\hline Navigation condition & 5 & 5.4887 & 10.13 & 0.0001 \\
\hline Navigation condition X Subjects & 144 & 0.5417 & & \\
\hline \multicolumn{5}{|c|}{ Workload Ratings For Time Stress } \\
\hline Source & $\mathrm{dF}$ & MS & $\mathrm{F}$ & $\mathrm{P}$ \\
\hline Navigation condition & 5 & 3.9093 & 7.5 & 0.0001 \\
\hline Navigation condition X Subjects & 144 & 0.5211 & & \\
\hline \multicolumn{5}{|c|}{ Workload Ratings For Visual Effort } \\
\hline Source & $\mathrm{dF}$ & MS & $\mathrm{F}$ & $\mathrm{P}$ \\
\hline Navigation condition & 5 & 8.5755 & 10.89 & 0.0001 \\
\hline Navigation condition X Subjects & 144 & 0.7875 & & \\
\hline \multicolumn{5}{|c|}{ Workload Ratings For Psychological Stress } \\
\hline Source & $\mathrm{dF}$ & MS & $\mathrm{F}$ & $\mathrm{P}$ \\
\hline Navigation condition & 5 & 5.0414 & 7.93 & 0.0001 \\
\hline Navigation condition X Subjects & 144 & 0.6358 & & \\
\hline
\end{tabular}

The summed subjective workload measure across display type is shown in figure 28. As indicated by the SMM analysis, the paper map and route map without voice conditions had a higher overall workload rating than the three remaining TravTek conditions. The paper direction list had a significantly lower value than the paper map but not the route map without voice condition. 


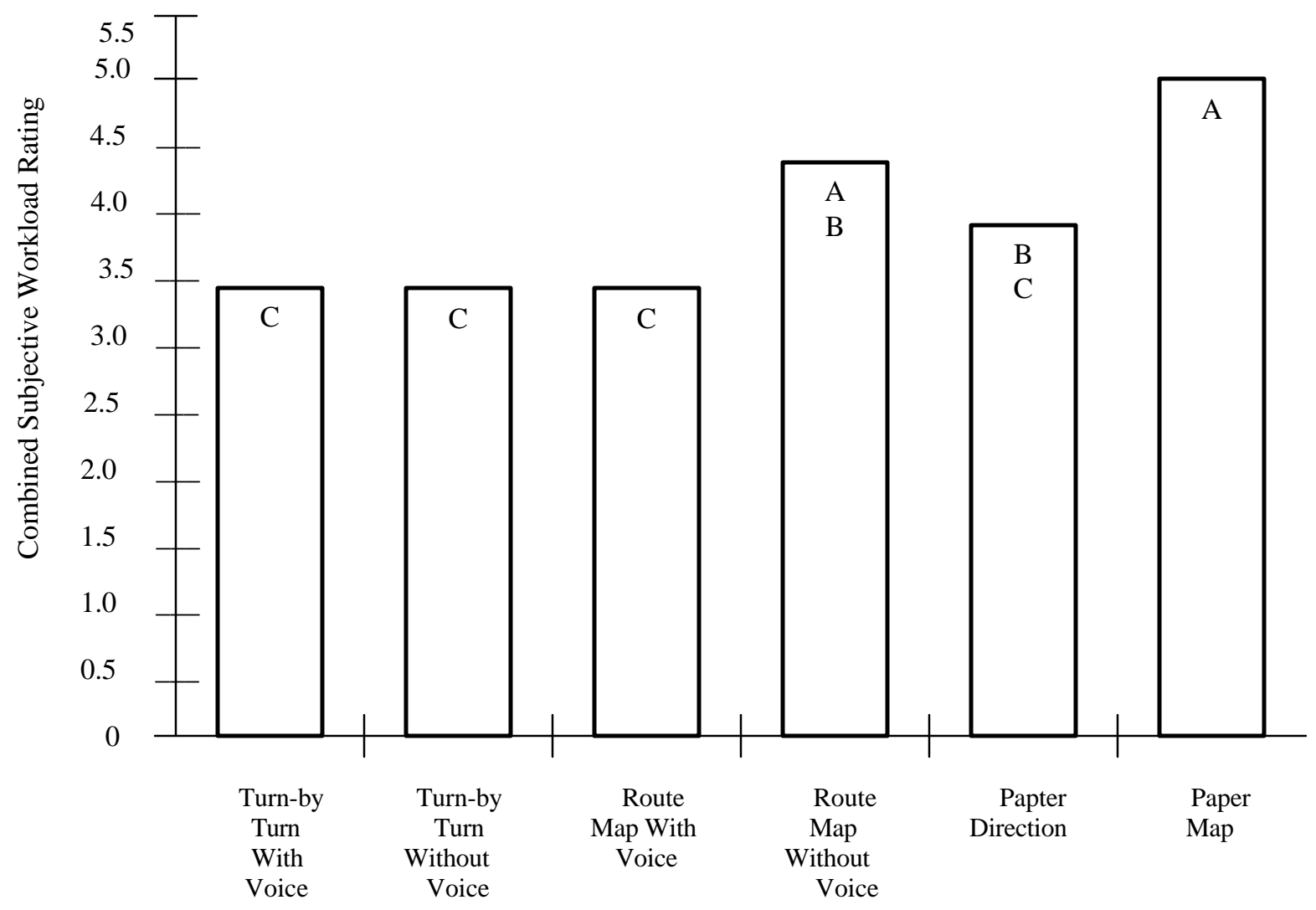

Figure 28. Total of all three subjective workload rating categories for each navigation condition.

The mean values and SMM comparisons for the three individual subjective workload dimensions are shown in figures 29,30, and 31. With few exceptions, each of the three subjective workload dimensions are highly correlated. In general, the paper map had the highest workload ratings, but these were not significantly different than the routemap without voice condition. This finding provides a key tie to the previous results of tlns section. Although the paper map condition required relatively low visual attention, it did apparently require a high amount of cognitive attention or workload. This is consistent with the hypothesis discussed previously in this section that drivers were attempting to hold information about the route in memory.

\section{Discussion}

The eye glance data revealed several interesting findings. First, overall the paper map condition required the least amount of visual attention. "Visual attention" in this context is operationally defined as the total amount of eyesoff-of-the-road time required to navigate to a destination. Note that drivers were instructed to write down their routes during the paper map condition and most drivers used the generated list to navigate. The generated list simulated a relatively common navigation practice and allowed the in-vehicle experimenter to determine if the driver was off the planned route. The task of generating the list of directions may have encouraged drivers to actively think about and memorize all or a portion of their routes. Subjects were also, with some success, able to develop a cognitive layout of the route which required less supplemental information from the navigation display. Second, the route-map presentation intrudes into the driving task more than the turn-by-turn presentation. This is apparent from the results showing twice as many glances occurred that were between 2.0 and $3.0 \mathrm{~s}$ for the route map conditions relative to their turn- by-turn counterparts. Third, the TravTek conditions which presented information without voice intrudes into the driving task more than the presentations with voice. The addition of voice in both cases, reduced the visual attention requirements in terms of both number and duration of glances. In this respect, voice guidance was clearly beneficial to navigating drivers. Fourth, an increase in visual attention by a navigation condition drew attention 
from the forward-left and forward-right roadways. In some cases, the reduction inisualattention being directed towards these locations could potentially detract valuableaccident avoidance visual resources from where they could be most effectively directed.

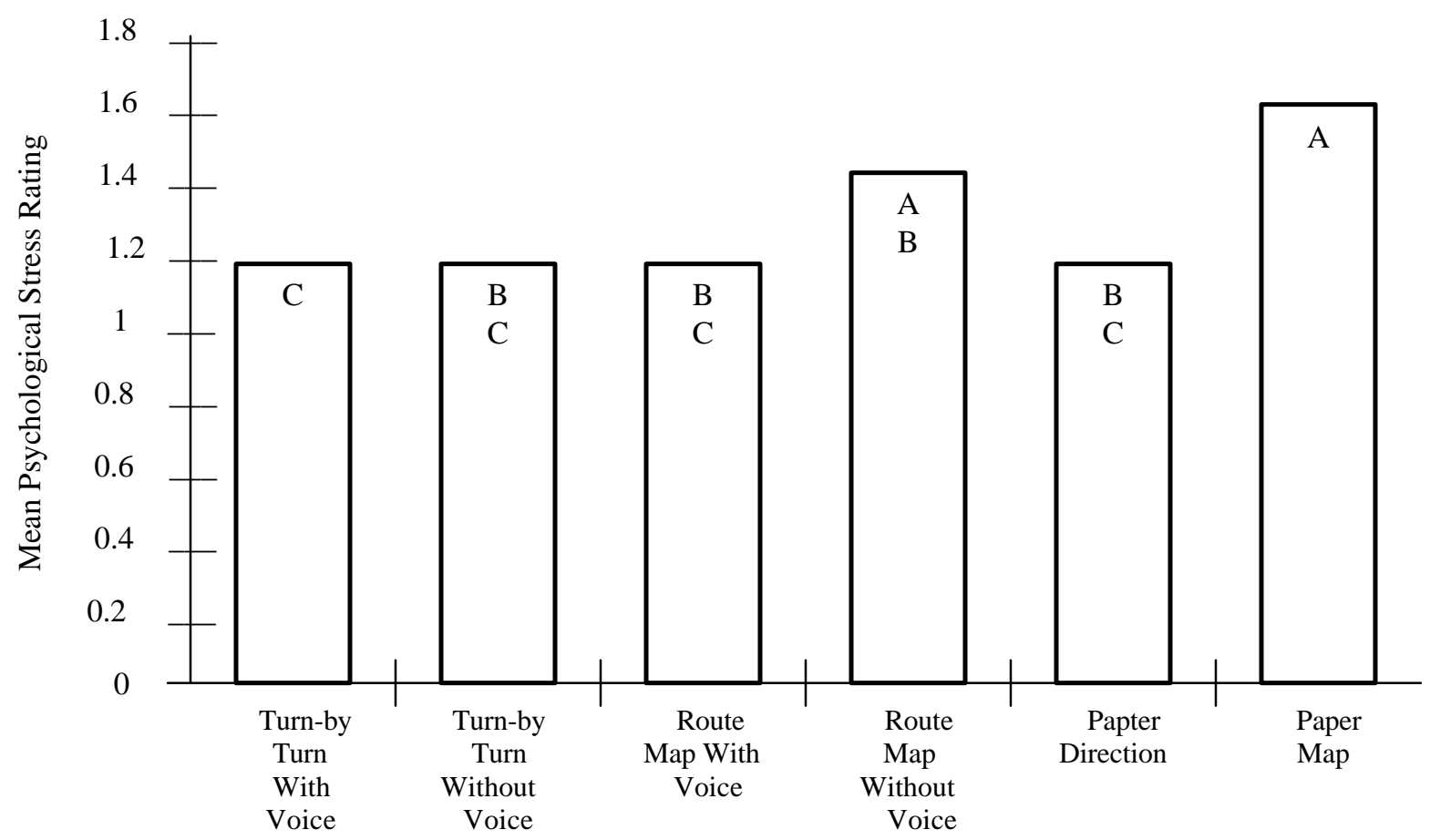

Figure 29. Subjective workload ratings of psychological stress for each navigation condition.

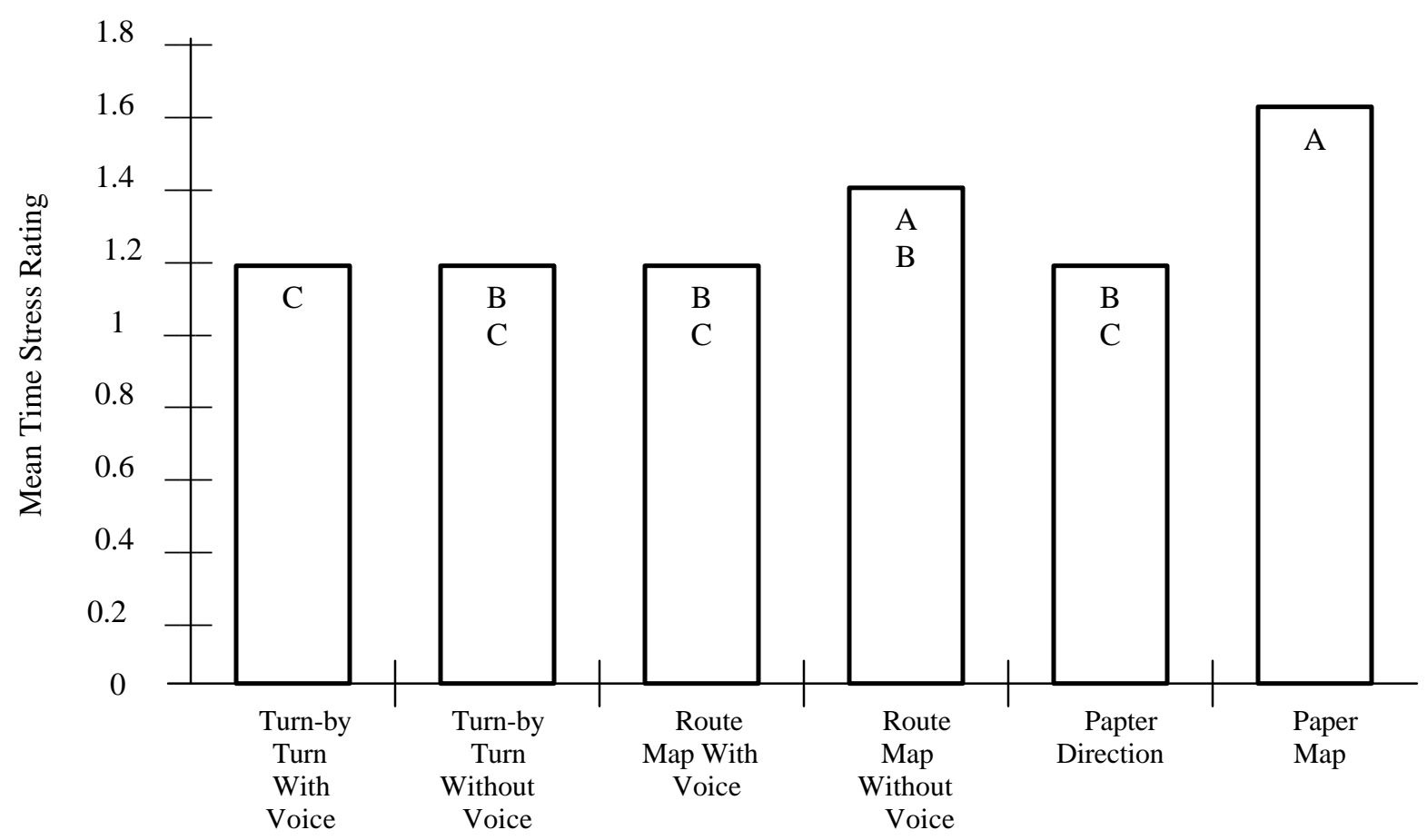

Figure 30. Subjective workload ratings of time stress for each navigation condition 


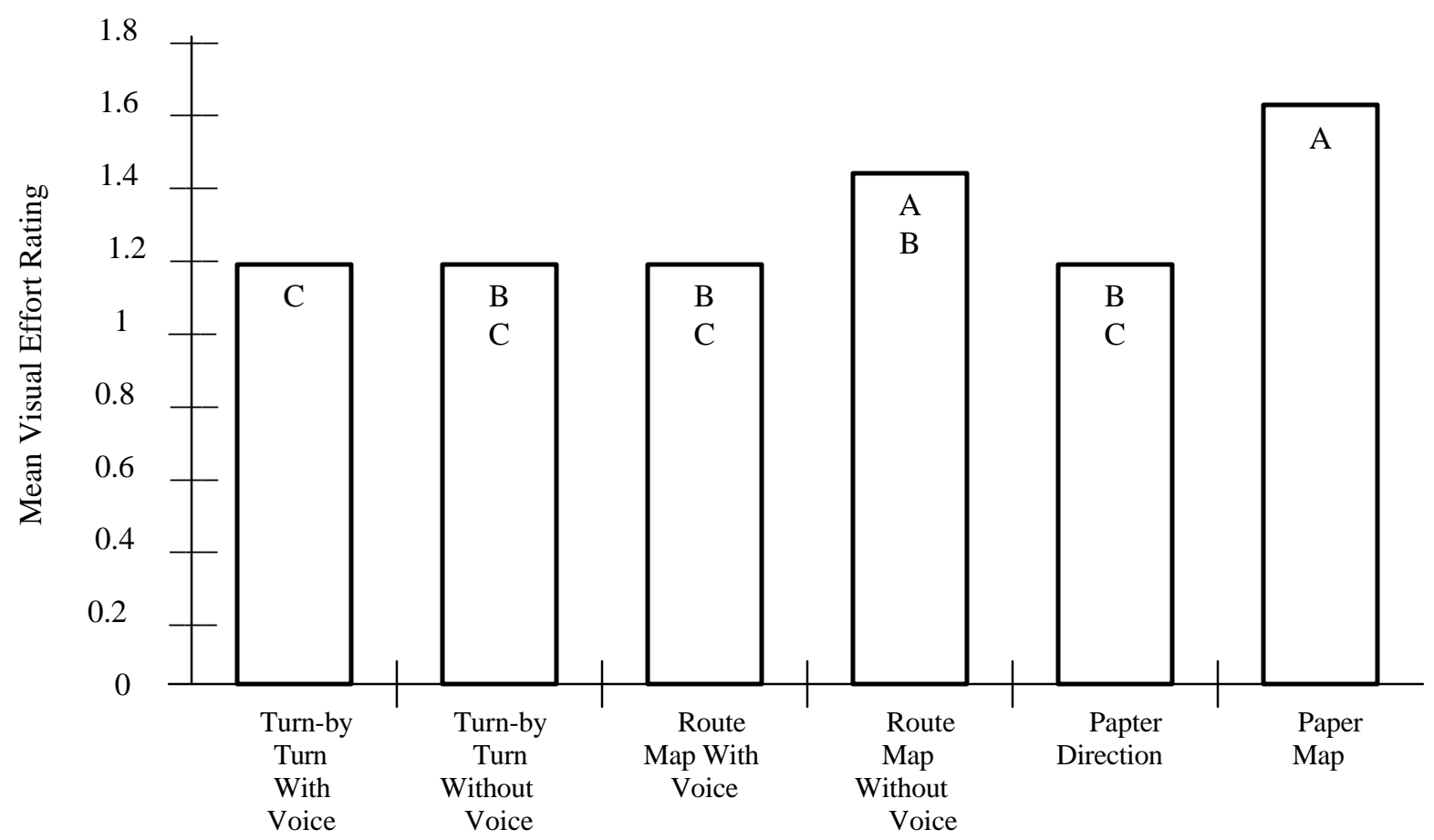

Figure 3 1. Subjective workload ratings of visual effort for each navigation condition.

Overall, few significant differences were found between navigation conditions with respect to lateral vehicle control, with the exception of the number of lane deviations. The turn-by- turn without voice and turn-by-turn with voice conditions had somewhat fewer numbers of deviations compared with the remaining four conditions. The TravTek turn-by-turn conditions had relatively short single display glance times, although these were only statistically different between the turn-by-turn with voice and the paper map, paper direction and route map without voice conditions. Single display glance time has been shown in previous research to correlate with lane position variance.(25)

The workload measures indicated that the paper map and route map without voice conditions were the most difficult to use. In considering the eye glance and driving performance data, it is apparent that the highest visual attention demand is created by the route map without voice condition. This is evident by both the number of glances and the single display glance length. Relatively speaking, drivers were required to look longer at the display to retrieve the required information. This demand is substantially reduced with the addition of voice guidance in conjunction with the route map display.

In contrast, the paper map condition required little visual attention in terms of number of glances, although the average single display glance time was comparable to the paper directions and route map without voice conditions and was higher than the turn-by-turn with voice condition. In addition, the number of abrupt braking maneuvers, mean speed, and workload ratings indicated that the paper map intruded upon the primary task of driving. This condition required high cognitive attention or workload. As will be discussed in the next section, the apparently high workload generated by the paper map caused drivers to stop and become lost more often relative to the other conditions. 


\title{
ISSUE 2: WHICH NAVIGATION CONFIGURATION(S) RESULTED IN THE BEST NAVIGATION PERFORMANCE?
}

\author{
Measures of Performance for Navigation Performance
}

Table 10 shows the ANOVA's conducted to determine whether any navigation conditions differed for the measures of navigation performance. As shown in the table, variables which were significant at the $\mathrm{p}<0.05$ level were: Time required to plan a trip, time required to drive to the destination, total time required to plan and drive to the destination, average number of stops while driving to the destination, and the amount of time the vehicle was stopped while driving to the destination. Note that no analysis or table is presented describing whether drivers arrived at their destination, because in all cases, drivers reached their destinations. In some cases, however, drivers took over an hour to complete what was designed to be a 20 min trip.

\section{Time Required to Plan a Trip}

Figure 32 shows the means and SMM post-hoc analysis results for the time required to plan a trip for each navigation condition. As shown, one of the control conditions, the paper map, was significantly different from all other navigation conditions and had the longest planning time. Subjects spent roughly 13 min on average studying the paper map compared to approximately 2.0 to $2.5 \mathrm{~min}$ for the other conditions. None of the other conditions were significantly different from one another. Note that the paper direction list condition did not, in actuality, have a trip planning element associated with its use. Recall that the subject was simply handed a direction list at the beginning of the drive. Although the subjects often studied the list momentarily, they were not required to input a destination and wait for the computer to generate a route (as with the TravTek conditions) or plan a route between two points (as with the paper map). Therefore, trip planning comparisons between the direction list and other conditions is not an accurate comparison.

\section{Time Required to Drive to a Destination.}

The means for each navigation condition and SMM analysis for time required to drive to a destination are shown in figure 33. This figure reveals that the turn-by-turn with voice had significantly shorter en route times than the route map without voice and paper map conditions. The turn-by-turn without voice, route map with voice and paper direction list conditions had significantly shorter en route times than the paper map control condition. As noted in the previous section, drivers drove slower while using the paper map. The other navigation conditions were not significantly different from one another.

\section{Total Required Trip Time}

When the trip planning and travel time data were totaled to establish the amount of time required to plan and drive to a destination (see figure 34), the paper map control condition was significantly different from all other navigation conditions. Again, the paper map condition required the longest total time; an average of over 13 min longer than the next longest time to plan and drive to a destination designed to be $20 \mathrm{~min}$ en route.

\section{Number of Stops}

The results for the average number of stops for each navigation condition are shown in figure 35 . The paper map condition was not statistically different from route map without voice and paper direction list conditions. However, it was significantly different and had a larger number of stops (on average) than the remaining TravTek conditions. The route map 
with voice, turn-by-turn without voice, and turn-by-turn with voice were not significantly different than the route map without voice and paper direction conditions.

Table 10. ANOVA's for navigation performance.

\begin{tabular}{|c|c|c|c|c|}
\hline \multicolumn{5}{|l|}{ Time Required to Plan a Trip } \\
\hline Source & $\mathrm{dF}$ & MS & $\mathrm{F}$ & $\mathrm{P}$ \\
\hline Navigation condition & 5 & 2136030.0 & 83.59 & 0.0001 \\
\hline Navigation condition X Subjects & 136 & 30190.91 & & \\
\hline \multicolumn{5}{|c|}{ Time Required to Drive to a Destination } \\
\hline Source & $\mathrm{dF}$ & MS & $\mathrm{F}$ & $\mathrm{P}$ \\
\hline Navigation condition & 5 & 451700.35 & 5.66 & 0.0001 \\
\hline Navigation condition X Subjects & 136 & 79829.7 & & \\
\hline \multicolumn{5}{|c|}{ Time Required to Plan and Drive to a Destination } \\
\hline Source & $\mathrm{dF}$ & MS & $\mathrm{F}$ & $\mathrm{P}$ \\
\hline Navigation condition & 5 & 4213760.1 & 39.29 & 0.0001 \\
\hline Navigation condition X Subjects & 136 & 107246.8 & & \\
\hline \multicolumn{5}{|l|}{ Number of Stops } \\
\hline Source & $\mathrm{dF}$ & MS & $\mathrm{F}$ & $\mathrm{P}$ \\
\hline Navigation condition & 5 & 91.59 & 4.96 & 0.0003 \\
\hline Navigation condition X Subjects & 139 & 18.47 & & \\
\hline \multicolumn{5}{|l|}{ Mean Duration of Stops } \\
\hline Source & $\mathrm{dF}$ & MS & $\mathrm{F}$ & $\mathrm{P}$ \\
\hline Navigation condition & 5 & 151030.59 & 10.17 & 0.0001 \\
\hline Navigation condition X Subjects & 139 & 14853.17 & & \\
\hline
\end{tabular}

\section{Time Stopped During the Trip}

The paper map condition had the longest total amount of time stopped (see figure 36) but it was not statistically different from the route map without voice condition. The three remaining TravTek conditions had less time stopped than the paper map control condition. The in-vehicle experimenter noted that it was not unusual for drivers to stop to study the map during the paper map condition. This observation likely explains the large difference in time stopped for the paper map relative to the other conditions.

\section{Navigation Errors}

Figure 37 shows the number of navigation errors for each of the navigation conditions. Four different types of navigation errors appear; lost, off-route, missed turn, and indecision about turn correctness. Lost errors include cases where, in the opinion of the in- vehicle experimenter, the drivers were completely lost for some period of time; however, all drivers eventually found their destinations. Off-route errors are operationally defined as circumstances where the driver strays from the planned route but corrects the error without getting completely lost. A missed turn is a situation where the driver is aware of an upcoming turn but misses it. This differs from the off-route case in that the driver immediately realizes that a turn was missed. Indecision about turn correctness refers to 
circumstances where a driver makes a correct turn decision, but was unsure for someperiod of time that the choice was correct.

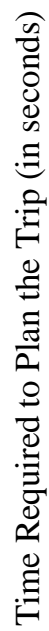

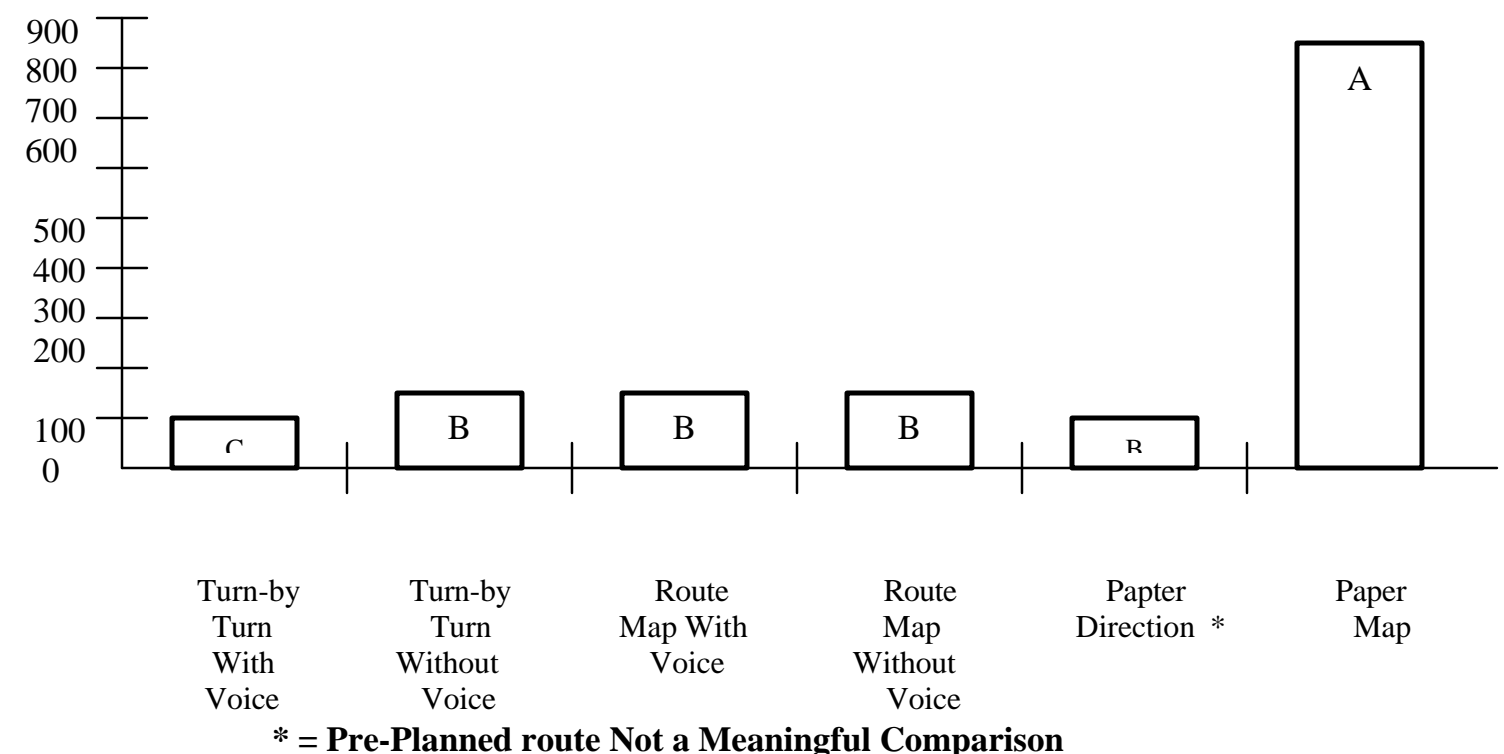

Figure 32. Time required to plan a trip for each navigation condition.

告

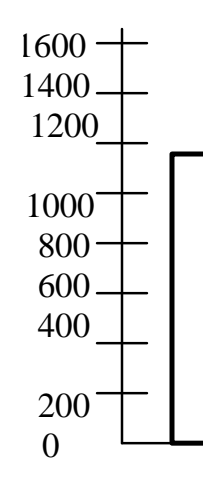

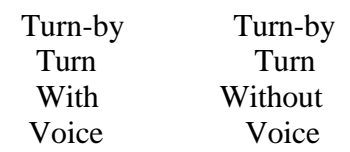
Route
Map With
Voice

A

Figure 33. Time required to drive to a destination for each navigation condition.

Figure 37, shows that a large number of missed turns were made when using the route map without voice condition. Over twice as many errors were recorded during this conditionthan any other navigation condition. The turn-by-turn with voice condition had the fewest number of missed turn occurrences. The in-vehicle experimenternoted, that particularly in cases where streets were close together or interchanges overlapped, it was difficult for thedrivers to determine exactly where to turn with the route map. 


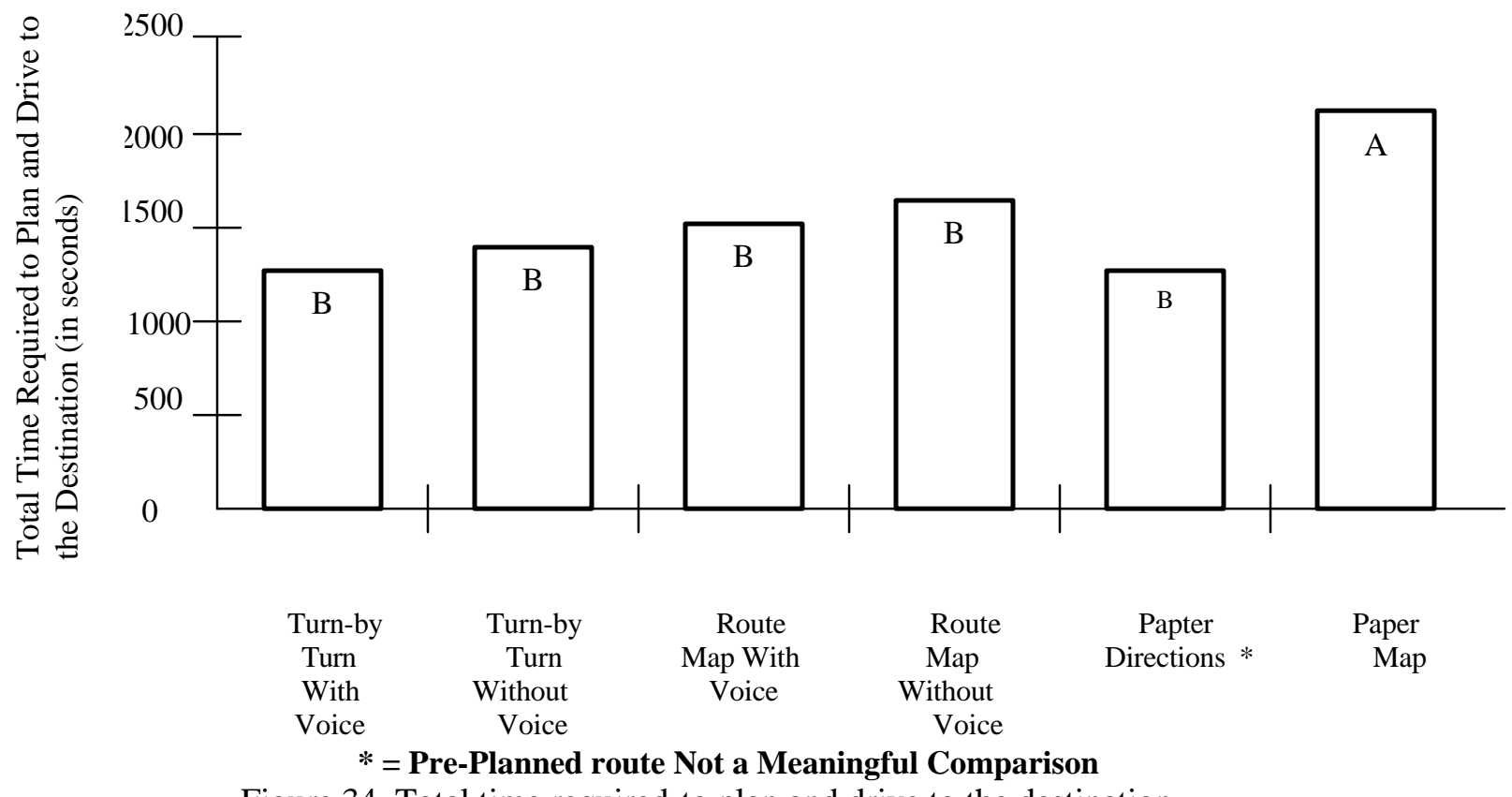

Figure 34 . Total time required to plan and drive to the destination

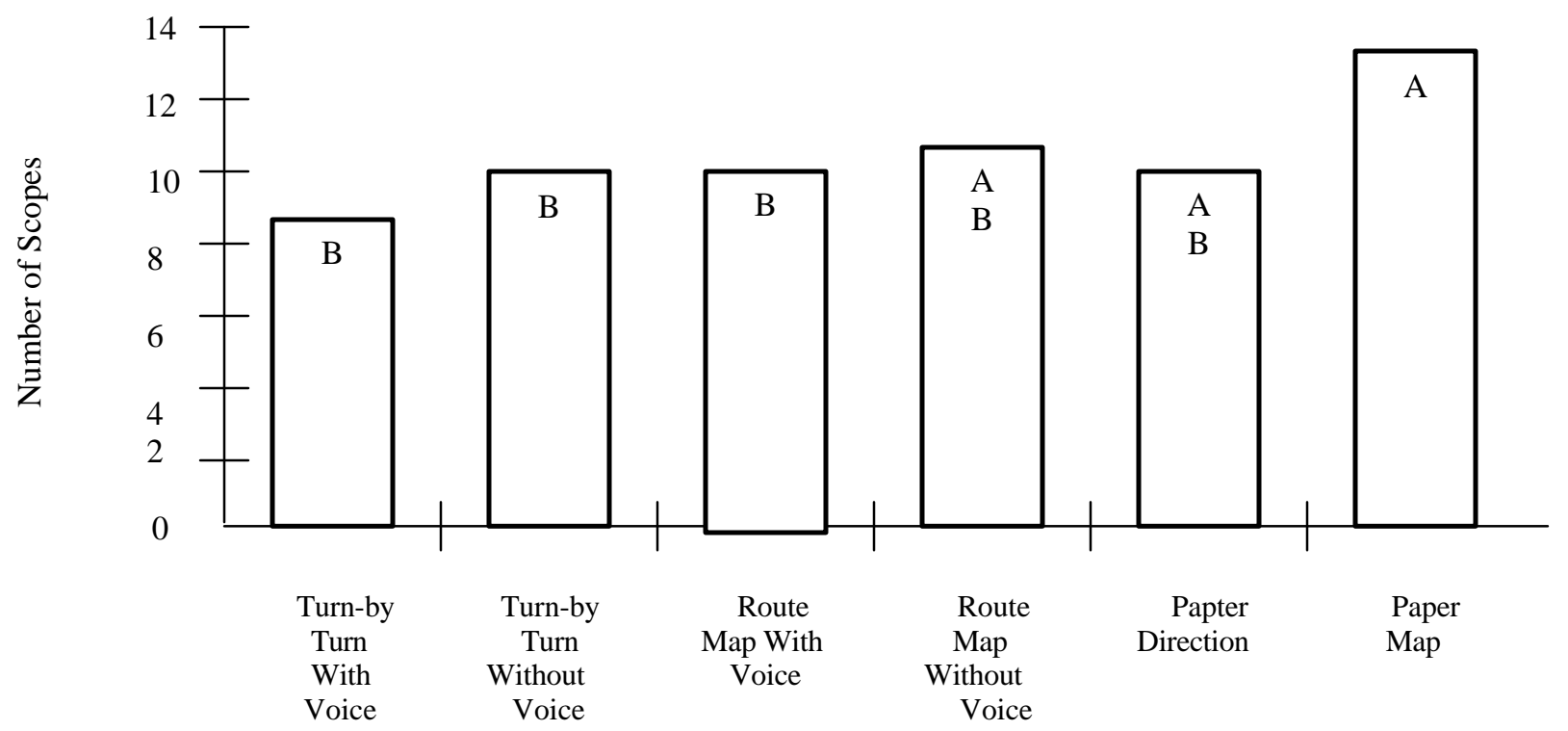

Figure 35. Number of stops made for each navigation condition.

The driver being lost was the most critical navigation error. For this situation, the paper map condition had the highest number of lost drivers, with 11. It is interesting to note, that the turn-byturn with voice condition had no instances where drivers were lost, while the route map with voice and paper direction list conditions had only two instances. As discussed in the previous section, drivers were apparently navigating more from memory in the paper map condition than in the other conditions. This reliance on memory was likely a causal factor in drivers becoming lost in this condition. 


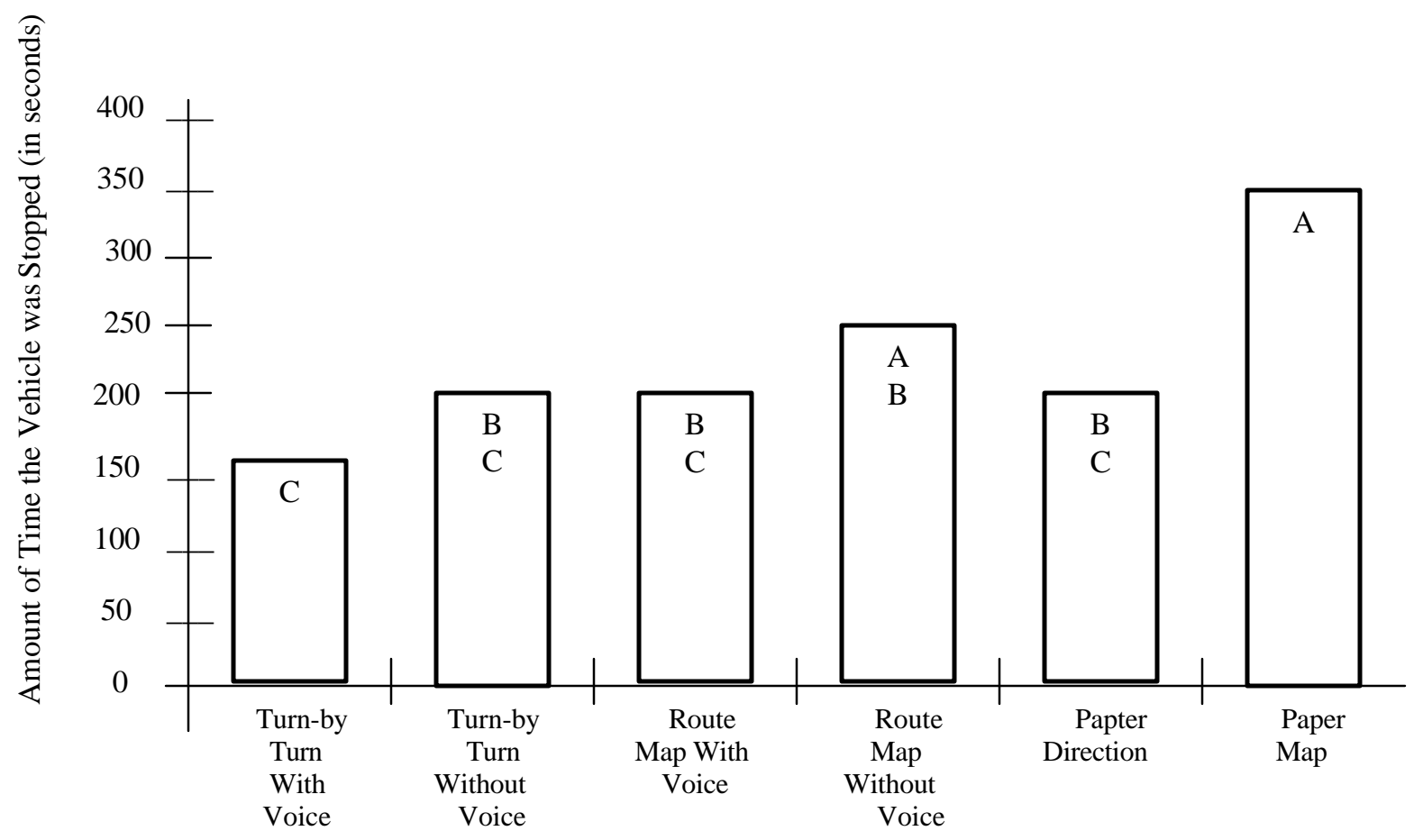

Figure 36. Amount of time the vehicle was stopped while driving to a destination for each navigation condition.

Figure 37 also shows the turn-by-turn conditions had a higher number of errors than the remaining conditions for the "indecision about turn correctness." This may be due to the lack of continuous transition information provided by the turn-by-turn visual display. That is, the route map rotates in a more continuous fashion than the turn-by-turn maps, which gives the driver information in real time. It should be noted that the TravTek design provided a function allowing the driver to switch between the turn-by-turn map and the route map when desired. This feature experience may mitigate this type of error. For the control conditions, the driver had to rely more on external stimuli and therefore may have been more aware of the correctness of the turn due to processing of street sign information, For the off-route category, the turn-by-turn without voice and the paper direction list resulted in the fewest errors. The paper map condition also had relatively few off-route incidents (see figure 37). The route map with voice had the largest number of off-route errors, although the reason for this finding is unclear.

\section{Discussion}

Relative to any of the other conditions, the navigation performance results revealed the TravTek configurations and the paper direction list resulted in better navigation performance than the paper map control condition, at least in terms of time required. In addition, drivers got lost the most while using the paper map control condition. It should be noted for the paper map control condition that drivers were asked to plan their routes and write the information down before they started driving. The drivers then referred to the map or the sheet of paper while driving to the destination. The in-vehicle experimenter noted that when the drivers became lost while driving with the paper map, they would usually stop the vehicle to study the map. This helps to explain why the paper map control condition had the longest plan and drive times. Note that workload was highest for the paper map and route map without voice conditions (see driving task intrusion section). Drivers reported to the in-vehicle experimenter that they felt stress in these conditions because they were not always familiar with where they were driving or how to use the map 
effectively to get to their destination. Some drivers reported that there was too much information to gather while driving under the paper map and route map without voice conditions. This caused confusion and errors in navigation. The drivers missed more turns for the route map without voice condition often because they were on too high of a zoom level. Recall that while at a higher zoom level, the system did not provide the same level of detail as the lower zoom levels. Overall, drivers reported that the turn-by-turn with voice produced the least amount of workload. This was consistent with the navigation performance results that showed few navigation errors, and short planning and travel times with this condition.

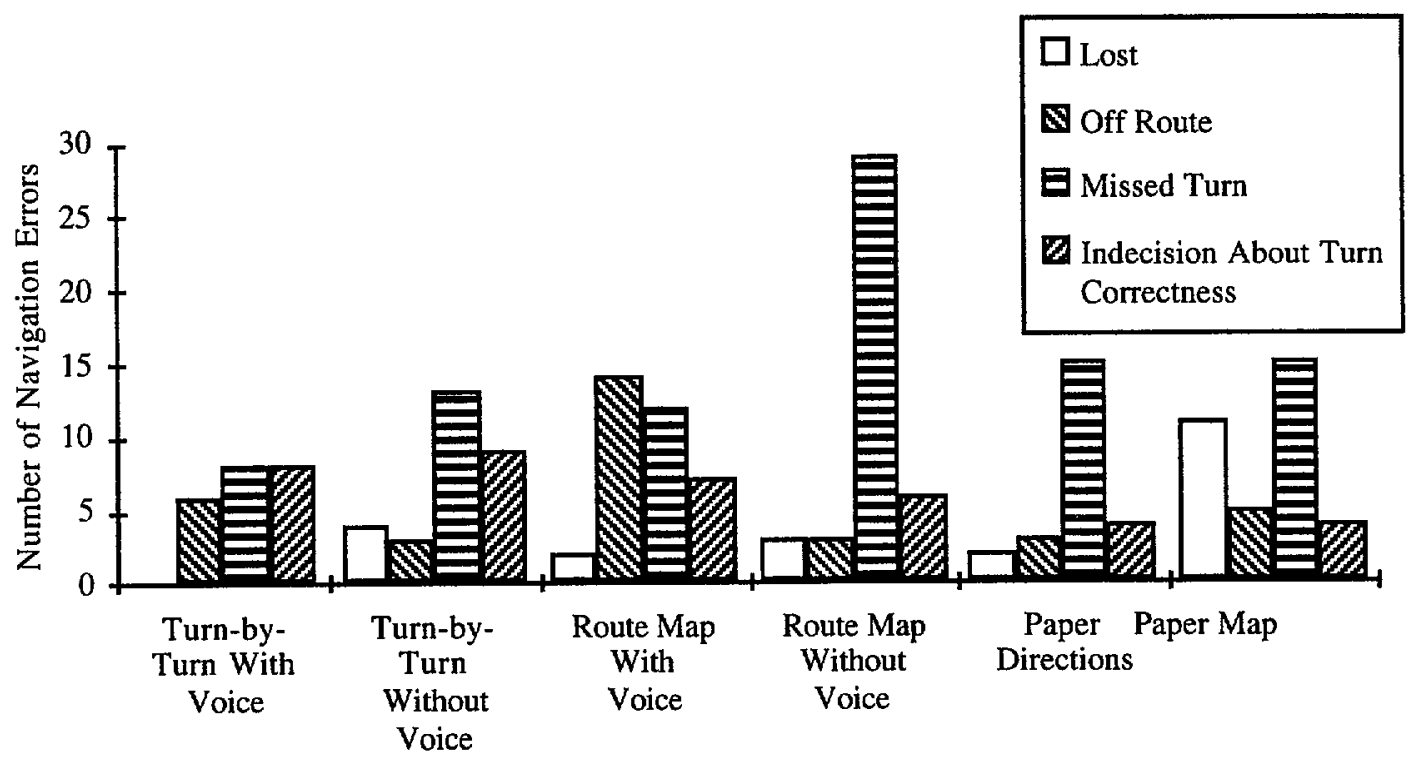

Figure 37. Number of navigation errors for each navigation condition.

The paper direction control condition required relatively short planning and driving times, as well as low workload levels. For this condition, drivers were given directions where to go instead of having to compose the directions themselves or input a destination. The paper directions simulated a computer-generated list and provided distances to each turn in a large legible font. In general, the key to these results appears to be that some method of effective turn-by-turn information significantly improves navigation performance. It is less critical whether this information is provided in a textual list (which could easily be provided on a CRT display), turn-by-turn graphic, and/or voice instruction. Navigation performance suffered when such information was not provided effectively (e.g., paper map performance suffered as indicated by both time and errors, and route map without voice performance suffered as indicated by the error data).

There was little variance in navigation performance between the turn-by-turn conditions. Apparently drivers were able to understand the required turn-by-turn information to get to the destination efficiently and effectively, since there was usable view-action information for each required decision point along the route. The route map with voice had a relatively high number of errors in two categories, which indicates that this combination may not be the most effective for navigation performance. This may be because there is more uncritical screen information that the driver is required to process. Note, however, that the route map was rated highly by drivers on questionnaires in other TravTek studies. It may be that 
when given a choice, a route map is a valuable addition to a system in some circumstances and/or for some users.

\section{ISSUE 3. CAN ANY OF THE TRAVTEK CONFIGURATION DESIGNS BE IMPROVED?}

A primary objective for the Camera Car Study is an evaluation of user interface designs for each of the four configurations. This evaluation includes a usability test and assessment of the ease of learning for the configuration used.

The following is a list of performance measures used to evaluate the usability and ease of learning:

- Time required to plan a trip.

- Time required to drive to the destination.

- Total planning and driving time to reach destination.

- Duration of glances for each navigation method.

- Number of glances for each navigation method.

- Amount of stop time during O/D drive.

- Number of stops during O/D drive.

- Number of missed turns.

- Number of times off route.

- Number of incidents of becoming lost.

This section will assess specific aspects of these performance measures which aid in distinguishing between usability and ease of learning. To enhance the usability of this report, figures from other sections have been included in this section. All of the figures selected represent findings that were statistically significant. Statistical tables, however, are not repeated here.

\section{Measures of Performance Applicable to Usability \\ Time Required to Plan a Trip}

Figure 38 shows the means and SMM analysis for time required to plan a trip. As shown, the paper map condition had significantly longer planning times than all other navigation conditions. These results indicate that the route planning feature for TravTek had a considerable time benefit relative to the paper map control condition. Recall from the previous section that the paper direction list did not actually require trip planning, since the list was simply handed to the subject at the beginning of a run. In actuality, such a list would have to be generated by computer or by hand. In either case, additional trip planning time would be required. In addition, as shown by the paper map condition where subjects were allowed to create their own lists, the hand-made lists would rarely be of equivalent quality to the direction list provided. Similarly, computer-generated lists would have to be created with a particularly large and legible font in order to be as usable as the list provided in this study.

\section{Time Required to Drive to the Destination}

The means and SMM analysis for time required to drive to the destination (shown in figure 39), revealed that the paper map control and route map without voice conditions required a longer amount of time to navigate to a destination than the turn-by-turn with voice condition. The turn-by-turn without voice, route map with voice and paper direction list conditions had significantly shorter en route times than the paper map control condition. 
The other conditions were not significantly different from one another. The in-vehiclexperimenter noted that the longer trip duration in the paper map configuration wasgenerally due to drivers selecting longernonfreeway routes, and getting lost more often; which led to more time stopped.

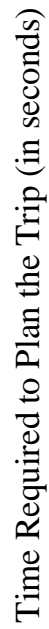

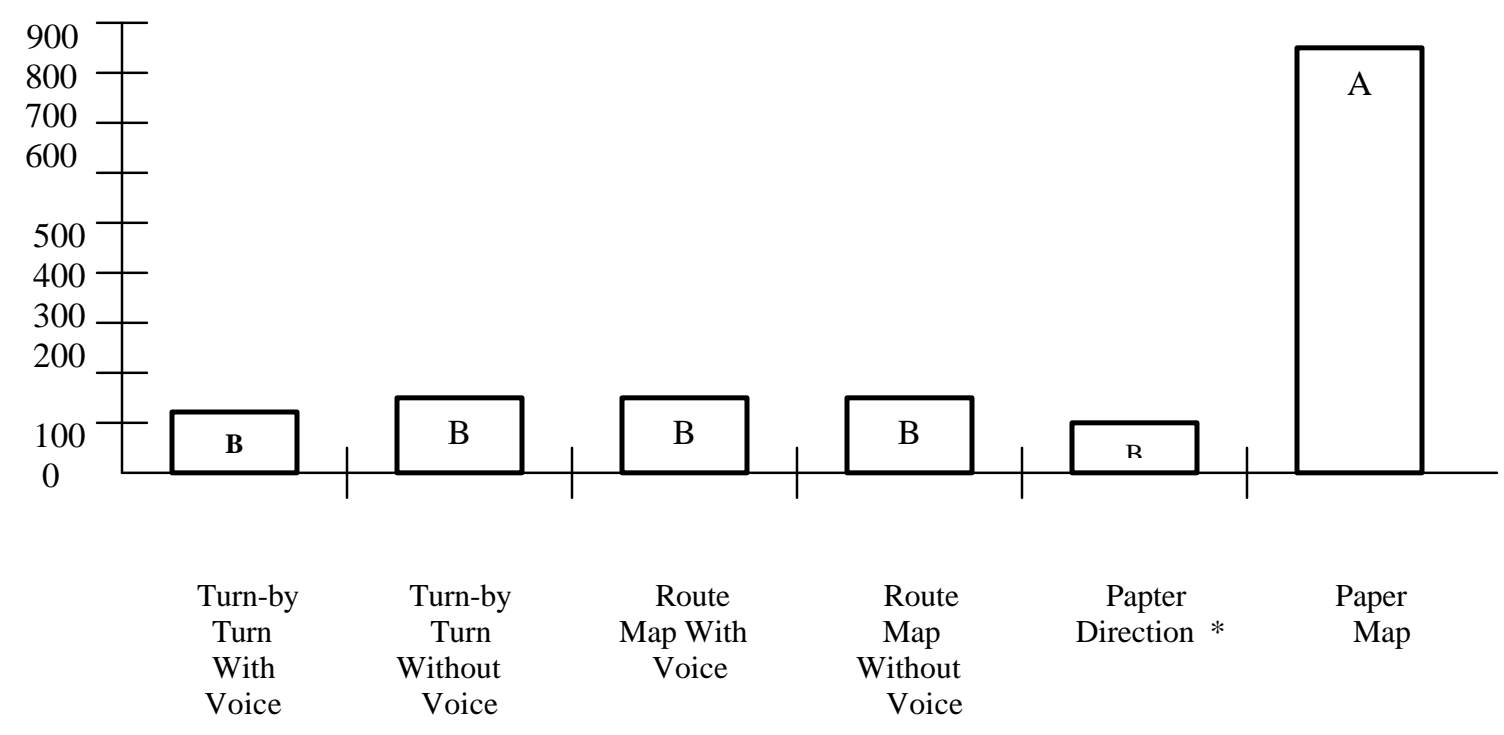

* = Pre-Planned route Not a Meaningful Comparison

Figure 38. Time required to plan a trip for each navigation condition.

.

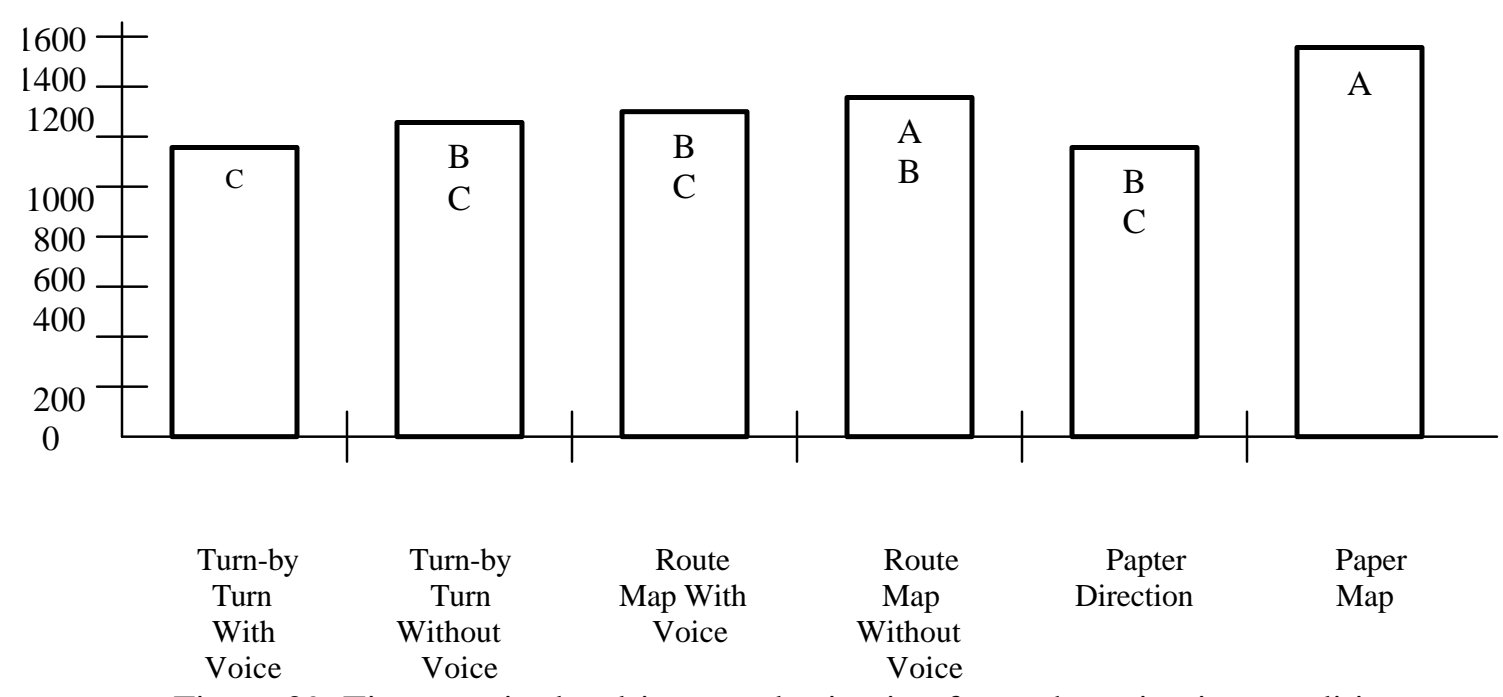

Figure 39. Time required to drive to a destination for each navigation condition. 


\section{Duration of Glances by Navigation Method}

The means and SMM post-hoc analyses for single glance duration indicate that the routemap without voice, direction list, and paper map have significantly longer glance times than the turn-by-turn with voice condition (see figure 40).

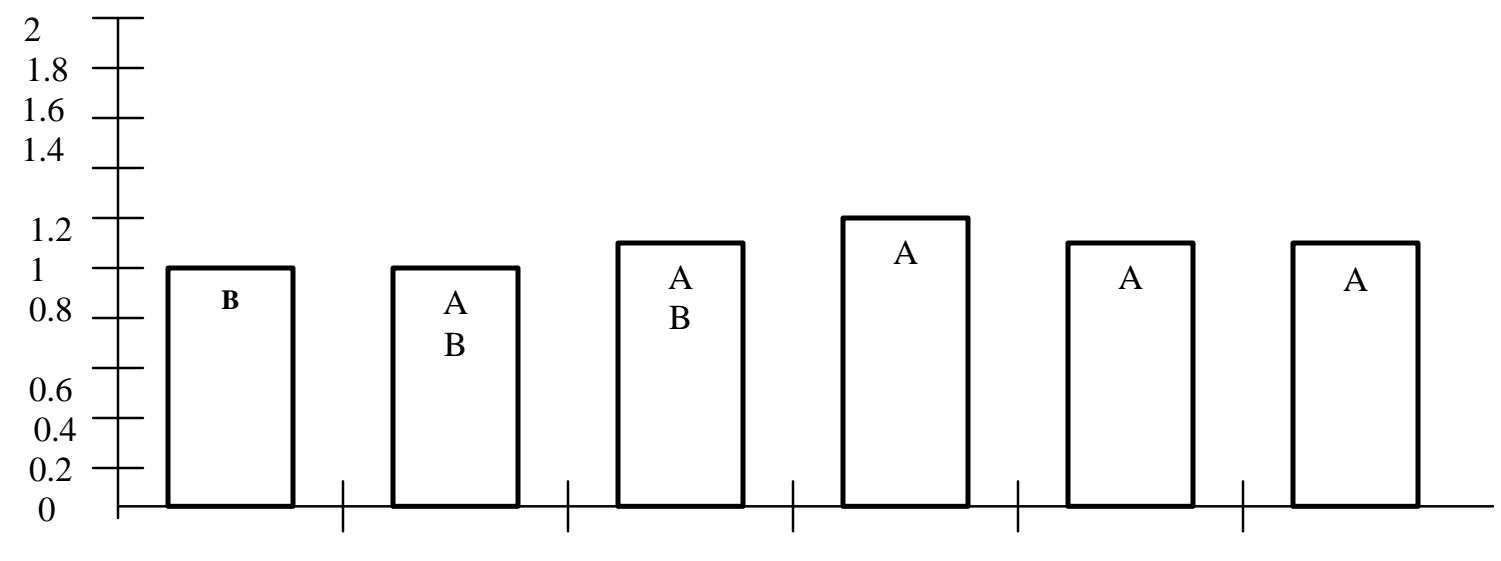

$\begin{array}{cccccc}\text { Turn-by } & \text { Turn-by } & \text { Route } & \text { Route } & \text { Papter } & \text { Paper } \\ \text { Turn } & \text { Turn } & \text { Map With } & \text { Map } & \text { Direction } & \text { Map } \\ \text { With } & \text { Without } & \text { Voice } & \text { Without } & & \\ \text { Voice } & \text { Voice } & & \text { Voice } & & \end{array}$

Figure 40. Duration of glances for each navigation condition; only glances at navigation display included.

Short glance durations indicate that drivers can gather information in the turn-by-turn with voice condition more quickly than other configurations. This is most likely due to voice guidance increasing situation awareness together with the simple screen to gain or verify selected information. In contrast, the route map without voice had long glance times because of the complex information presented by the condition. The $0.8 \mathrm{~km}(1 / 2 \mathrm{mi})$ scale used as the route map default often resulted in difficult to read street names. This was particularly true on the freeways where several exits andclover leaves could overlap. The aerial perspective of the route map also sometimes made it difficult to determine where to exit.

\section{Number of Glances by Navigation Method}

The number of glances to the navigation aids ranked from highest to lowest by navigation condition are: route map without voice, turn-by-turn without voice, route map with voice, turn-by-turn with voice, direction list, and the paper map (see figure 41).

As noted in the previous section, the route map without voice also had long glance times. The number of glances reflects the increased attention demand required by this condition. From a usability perspective, this condition required the greatest degree of visual driver resources to gain the necessary navigation information. The non-voice condition of the turn-by-turn display had the second highest number of glances. Adding voice to the condition improved both the turn-by-turn and route map cases (probably due to the addition of a non-visual, redundant navigational information source). 


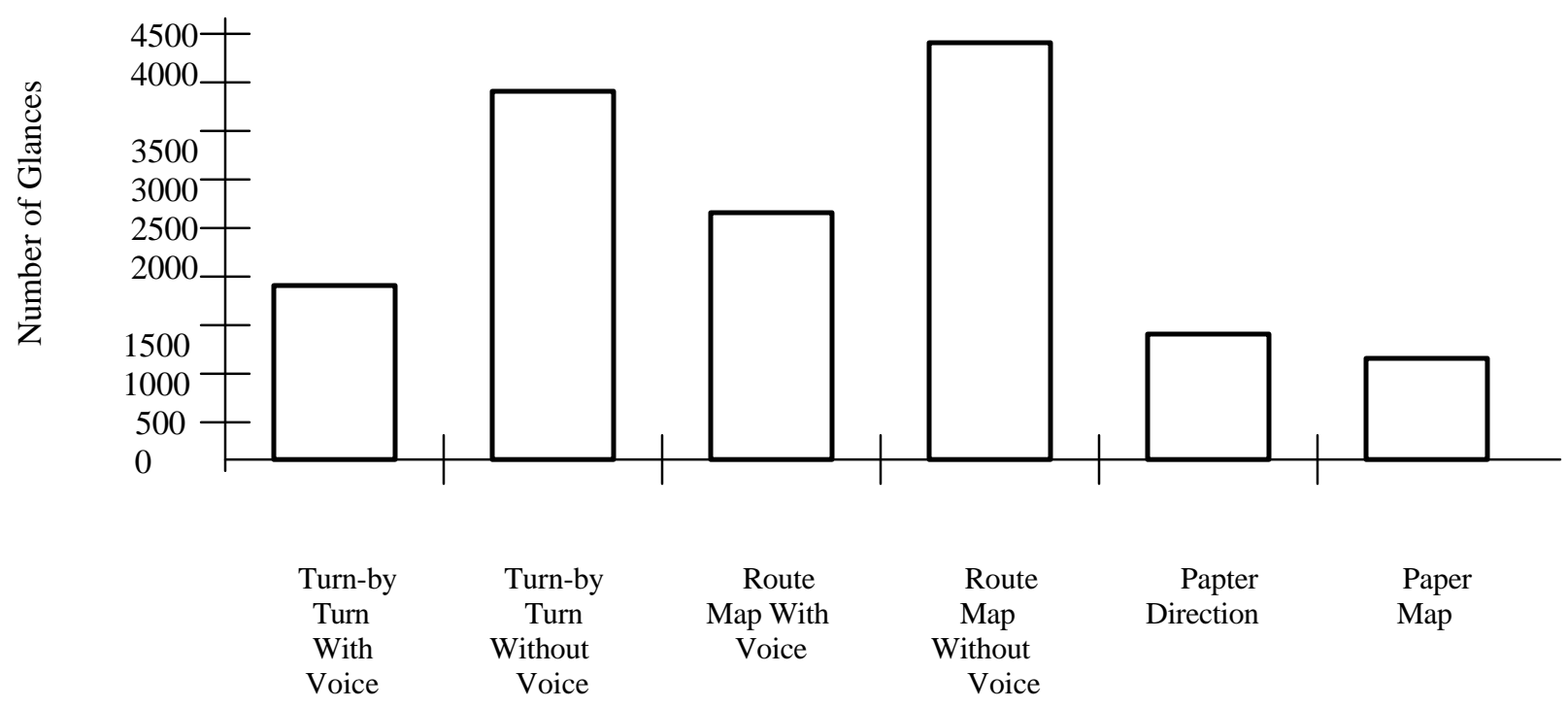

Figure 4 1.Number of glances to the navigation display for each navigation condition.

As was shown in the previous section, the low number of glances in the paper map condition was not indicative of navigation performance. It is important to note that drivers were invited to prepare a direction list for themselves prior to starting the O/D. This resulted in glances to their own direction list in place of, or in addition to, the paper map. Subjects' performances with their own list were quite poor. Subjects often wrote down incomplete directions for themselves and in some cases wrote down incorrect navigation information. This is in contrast to the paper direction condition, which consisted of legibly typed, organized, and reliable distance information. It appears that textual turn-by-turn instructions were as usable as a graphic presentation and could be included as a format option of an in-vehicle display. There is little reason to believe, from these results, that meaningful usability differences would be found.

\section{Amount of Stop Time While Navigating}

Drivers spent significantly more time stopped while using the paper map than all other navigation conditions with the exception of the route map without voice. The turn-by-turn without voice condition had a shorter time stopped than either the route map without voice or the paper map conditions (see figure 42). As shown in previous results, the differences in stopped time can be directly attributed to usability concerns; drivers becoming lost, missing turns, and generally being unsure of their navigation plan.

\section{Number of Stops}

The average number of stops that drivers made, indicated that the TravTek turn-by-turn conditions and the route map with voice had fewer stops than the paper map (see figure 43). This is consistent with previous results which indicated that the three TravTek conditions that provide turn-by-turn information either by graphic or voice or both are more usable than the paper map control condition. 


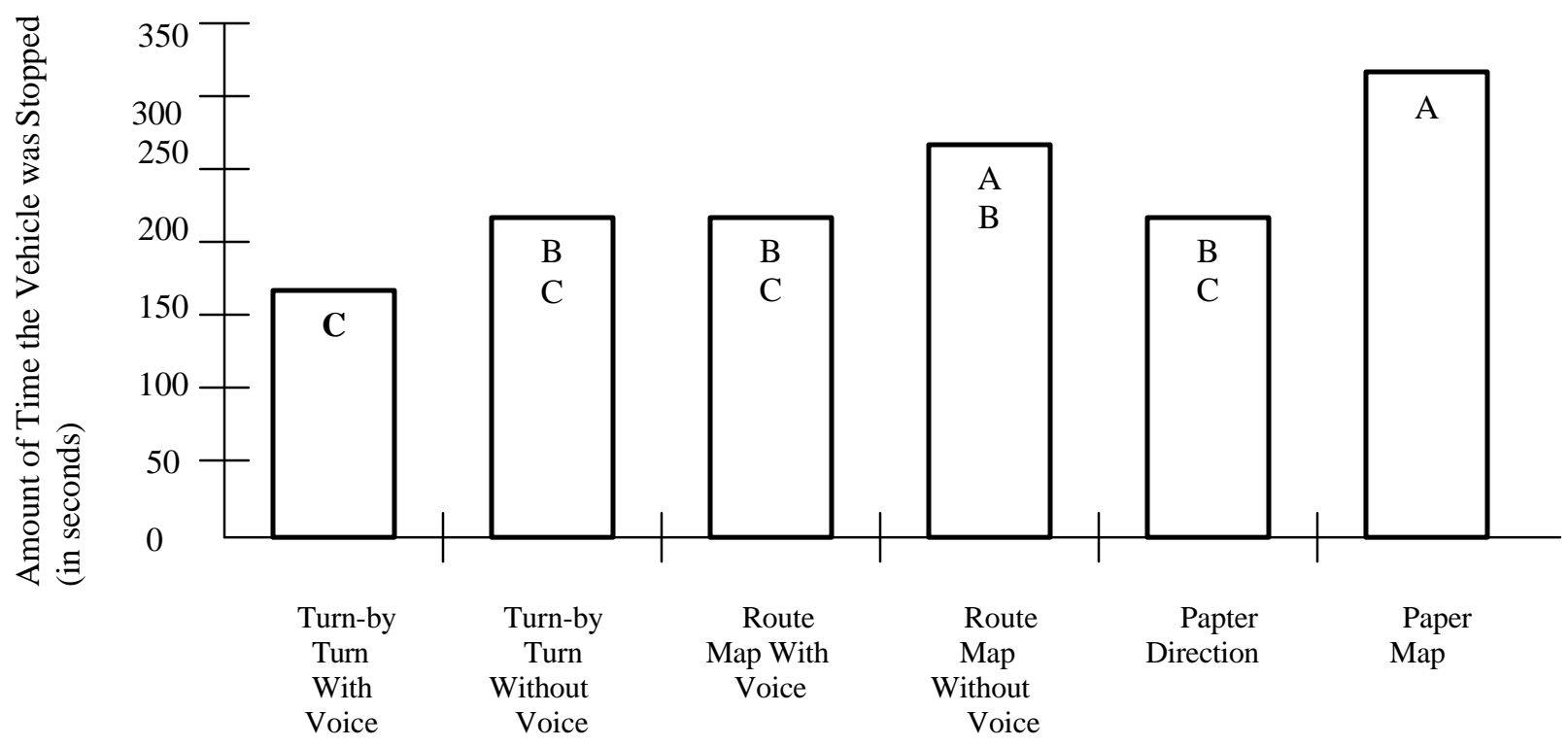

Figure 42. Amount of time the vehicle was stopped while en route to a destination for each navigation condition.

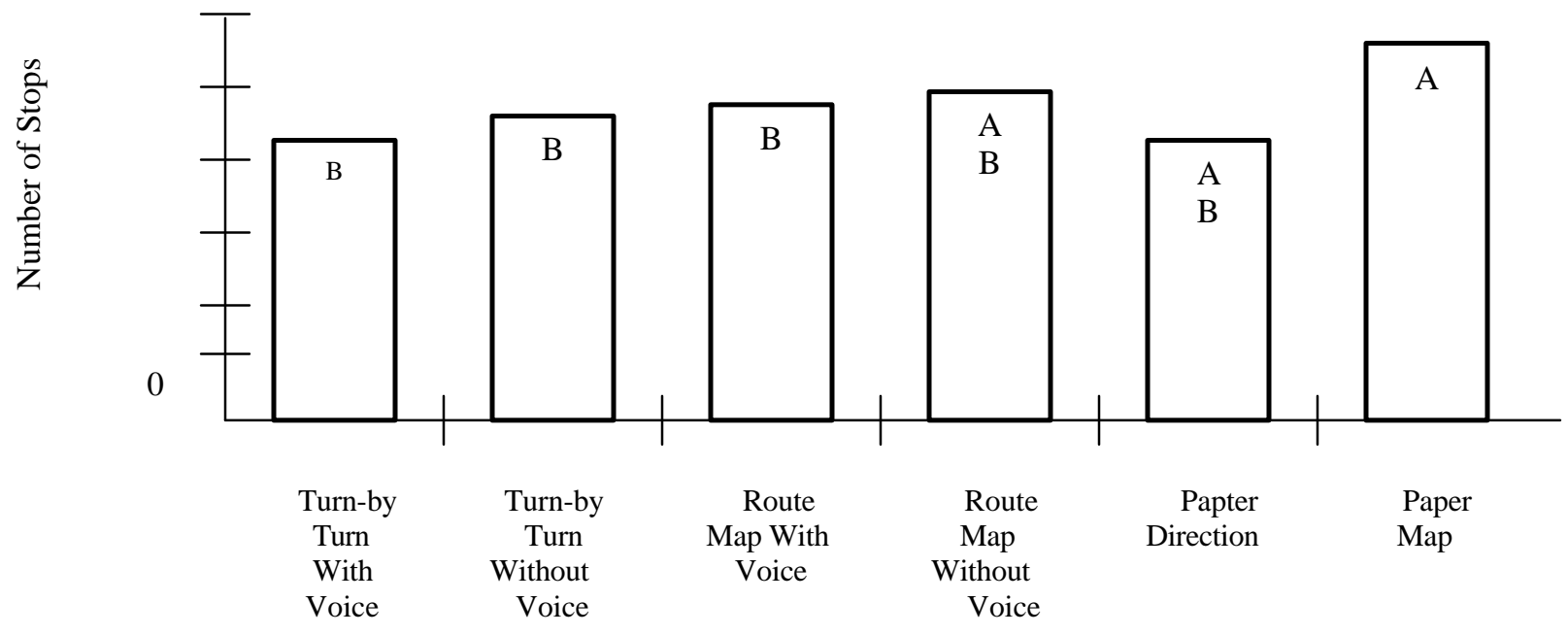

Figure 43. Average number of stops made during drive to a destination.

\section{Number of Missed Turns}

There were a higher number of missed turns in the route map without voice navigation condition. The experimenter noted that missed turns for this condition were due to a lack of map resolution and appearance of street names at the $0.8 \mathrm{~km}(1 / 2 \mathrm{mi})$ zoom level. Freeway exits also appeared to cause some difficulty. Most missed turns occurred while driving at highway speeds, where freeway exits appeared quickly and were difficult to see. 
Many freeway off-ramps looped under one another in the Orlando area and made it difficult to interpret the programmed route.

\section{Number of Incidents of Becoming Lost}

The paper map condition had the highest number of incidents of drivers becoming lost (11 occurrences in 42 drives). The other navigation conditions showed few incidents and the turn-by-turn with voice condition had no incidents of drivers becoming lost. For the paper map case, it was apparent that drivers could not effectively refer to the map while driving, and that their written instructions were not always helpful.

\section{Discussion of Usability}

Overall, test drivers who participated in the camera car study performed extremely well. The TravTek driver interface was easy to learn and use. This was true even for older drivers, some of whom had little or no computer experience. As reported earlier, older drivers especially benefited from TravTek when compared with the control condition.

The performance measure results indicate that the TravTek route guidance conditions were more usable than a paper map. The textual direction list with the entire route pre-planned and a large, readable font was as usable as any of the TravTek conditions. From a global perspective, the turn-by-turn guidance display with voice appears to be the easiest TravTek condition and the route map without voice appeared to be the most difficult TravTek condition. It should be noted that the turn-by-turn visual and voice guidance displays were part of the human factors engineering of the driver interface and were designated as default conditions. When entering route guidance, the turn-by-turn visual display and voice instructions were automatically on. Drivers could turn the voice off or toggle to the route map by using the steering wheel buttons.

Several interesting points were revealed in the last section regarding how drivers used TravTek and the other conventional navigational methods. It appears that navigation performance using any of the TravTek configurations was effective relative to the paper map control condition. Both trip and planning times were generally faster, stopped time was less, and drivers were lost less in the TravTek and paper direction conditions. In addition, high workload ratings for time stress, visual effort, and psychological stress were recorded with the paper map.

Of the TravTek navigation conditions, the route map without voice appeared to be the most difficult to use. The in-vehicle experimenter observed that this was due primarily to the difficulty drivers encountered identifying turns. Although, a magenta line showed the programmed route, drivers often passed turns because they did not realize they had arrived at the turn. This problem is related to the road network and was particularly troublesome at small, unmarked intersections. Another problem with the route map without voice occurred on the freeways. The $0.8 \mathrm{~km}(1 / 2 \mathrm{mi})$ default scale did not provide enough detail for drivers to read the programmed exit names. This was especially the case when there was a left turn after a right lane exit. The route map appeared to indicate a left turn. Drivers would sometimes get into the left lane ahead of time, only to discover that the exit was to the right. Drivers also had trouble identifying freeway exits when clover leaves were on the programmed route. This lack of detail, combined with freeway speeds, did not provide enough time for some drivers to react. The issue of appropriate detail and zoom level issue does not have an easy solution, however: The higher zoom scale results in more preview and less detail (i.e., shows major streets and $1.6 \mathrm{~km}$ (1 mi) around vehicle), but 
the lower zoom scale results in higher detail and less preview information (i.e., shows major and minor streets and $0.4 \mathrm{~km}(1 / 4 \mathrm{mi})$ around vehicle).

The "beeps" associated with alerting the driver of an impending freeway navigation maneuver were not intuitive to many drivers. Drivers were better able to understand the $1.6 \mathrm{~km}(1.0 \mathrm{mi})$ tone alert because the programmed route appeared sooner on the visual display. However, the $3.2 \mathrm{~km}(2.0 \mathrm{mi})$ alert did not provide drivers with timely maneuver information, Drivers expected to see a programmed turn appear sooner than it did. This situation could be remedied by using secondary alerts for drivers as they approach the exit.

The paper map condition required significantly more and longer stops than the TravTek conditions with the exception of route map without voice. However, in the case of the TravTek navigation configurations, the in-vehicle experimenter noted that occasional stops were due to drivers either becoming lost or off-route. Drivers almost always pulled off to the side of the road to replan their routes. It was the exception when drivers drove slowly and potentially blocked traffic while replanning. Long glances to the TravTek interface were observed when the driver pulled back onto the roadway while the system was still replanning a route. Drivers sometimes glanced at the screen during the 4 to 5 -s computer processing replanning sequence. This problem will be mitigated in the future since these long recalculation times will be significantly reduced with increases in computer processing speed.

Another interesting trend was the contrast between the voice and non-voice conditions. Although the measured differences were not always significant, the voice conditions provided an apparent increase in situation awareness over the non-voice conditions. The experimenter observed that novice drivers preferred the voice over the non-voice condition. Many local users reported that once the system was learned, the voice was no longer needed for routine driving. However, if any drivers programmed an unfamiliar route, they generally reported that the voice was turned back on.

With regard to which map presentation was preferred, comments made by the younger local users indicated that they frequently switched between the turn-by-turn route guidance and the route map. Drivers reported that the route map provided good route-planning awareness and the turn-by-turn guidance display provided good immediate turn guidance.

\section{Anecdotal Observations of Usability and Ease of Learning From the In-Vehicle Experimenter}

The camera car experimenter observed subjects learning and navigating with TravTek and the other navigation conditions throughout the driving trials. TravTek proved to be reliable and relatively easy to learn. Both younger and older drivers consistently performed better using TravTek and seemed to prefer it to the paper map control condition.

Younger drivers appeared to have less trouble adapting to the TravTek interface. It was apparent that they were more computer literate and were able to plug in the destination and go. Younger drivers also "played" with and explored the TravTek functions more than the older drivers. The older drivers learned more slowly, but typically caught on after a few practice sessions while idling the car. Training on the system with practice, lasted about 20 min for all users.

Many subjects that participated in the Camera Car Study had difficulty identifying screen buttons (those active areas on the VIC touch screen); specifically, the screen buttons next to street names. Subjects often stared blankly when confronted with what to do next. After learning how these buttons worked, several subjects commented that the screen buttons 
should look more like buttons. In addition to designing more salient buttons, button identification could be enhanced by providing prompting information for the driver. One recommendation is to have a two-tiered interface; one for novice users, which prompts the driver for additional information, and a second more streamlined interface for expert users.

Drivers also experienced a problem with the TravTek routing magenta line's "tail" during the camera car trials and in their own local users vehicle. This "tail" problem generally occurred when subjects became lost and turned around and started following the magenta path in the wrong direction. Then, when TravTek informed them that they were off route, they refused to believe the system because they were driving on the magenta line. This problem is easily remedied by trimming the tail at the bottom of the display.

Updating and computing a new route too slowly was a recurring problem. If a driver missed a turn and continued driving straight at a moderate speed, TravTek would give an off-route message. When the driver pressed the "replan" button, a problem arose when the new route was assigned. Often times, if the closest route required a U-turn, the TravTek routing magenta line showed a U-turn which had already passed through the bottom of the display. TravTek almost immediately issued another off-route message. This happened repeatedly in some cases. One solution is to build the vehicle speed and heading into the TravTek navigation algorithm, thereby predicting where the vehicle's position will be by the time it finishes recalculating the new route.

Often times, TravTek selects routes on roadways which have higher speed limits but have more traffic lights. This holds true for toll roads as well. Many drivers became frustrated after being routed onto a tollway and then immediately off again in a matter of minutes (or even several hundred meters in some cases). This occurred even when there were fast, no toll routes available. In some cases, TravTek bypassed a two-lane roadway with a speed limit of $65 \mathrm{~km} / \mathrm{h}(40 \mathrm{mi} / \mathrm{h}$ and placed a driver on an $83 \mathrm{~km} / \mathrm{h}(50 \mathrm{mi} / \mathrm{h}$ roadway with traffic signals every $0.4 \mathrm{~km}(0.25 \mathrm{mi})$ and heavy traffic. Local users were frustrated by this type of routing and often did not heed TravTek advice when they knew of other more efficient routes.

Local users perceived "real-time" traffic information provided by the interface in the navigation plus mode to be unreliable, since they perceived the update rates to be too slow. This again is due to the infrastructure of the Orlando traffic network and is not due to the TravTek interface design.

\section{Ideas and Suggestions for Future Systems}

An area that local users, as well as some visitors commented on, was the lack of toll stations in the navigation data base. Drivers wanted to know where the toll plazas were and how much they would cost prior to arriving at a toll. Although this is not an interface issue per se, it is information that would increase drivers situation awareness and confidence.

Several local users suggested that voice input of destinations be made available. They also stated that the input of destinations became cumbersome after a period of time. Thus, speaker dependent voice recognition should be considered for future systems.

Local users found it difficult to delete stored destinations. After a period of time, drivers wanted to delete stored destinations that were no longer needed. This was not easily done with the current TravTek interface.

High mileage professional drivers that participated in the Local Users and Camera Car studies also saw a need for entering a string of destinations for the day, and then having 
TravTek plan the most efficient route. This "itinerary" feature should also have a data link capability so that drivers could send destination information from their personal computers to the TravTek vehicle via cellular phone data link. This would cut down on the amount of planning time. One local user drove to 10 destinations per day in Orlando. She reported that she spent upwards of an hour each day planning her route so that she did not double back.

\section{Conclusions on Overall Safety and U sability}

TravTek provided a tangible safety benefit during the Camera Car Study. One local user described an incident that sold her on the system. While navigating to an unknown destination on the outskirts of Orlando on a rainy night, she came across an automobile crash and instinctively stopped to assist. After assessing the situation, she called 911 on her cellular phone to request paramedics to the scene, but did not know where she was. While still on the phone, she remembered that TravTek could provide her with this information, so she pressed the "where am I button" on the steering wheel. The synthesized voice reported the current intersection and the 911 operator overheard the speaker phone and dispatched help immediately. Under normal circumstances, the local user would have lost valuable time by having to drive to find the next intersection.

\section{ISSUE 4: DO ANY OF THE NAVIGATION CONFIGURATIONS RESULT IN UNSAFE DRIVING BEHAVIOR?}

In order to assess the effects of using TravTek configurations on driving safety, an analysis of near-miss events and safety-related driving errors was conducted. Ideally, accident data would be used to evaluate the safety issue, since they provide irrefutable evidence of an unsafe circumstance. During the course of the Camera Car Study, however, no accidents occurred. Fortunately, estimates of safety risk could be addressed by analyzing near misses and safety-related errors. It has been shown in other domains that near misses can be used to estimate the likelihood of an accident (see the issues section of this report). Unfortunately, no data are in existence which tie the relative probabilities of near misses and accidents together for driving. Overall, this methodology will be valuable for comparing the relative safety of the tested navigation conditions. Additional research will be required to determine accident rates for given levels of market penetration.

A systematic method of classification was developed in order to accommodate three major elements that contribute to the severity of safety-related driving errors. These elements included classifying the error by determining the type of potential accident and associated injury potential, noting the roadway and/or intersection type (including the likely speed of other vehicles), determining the camera car speed, and noting the presence or absence of other traffic or obstacles.

The process of event classification was initiated by placing the safety-related error into 1 of 10 broad categories:

- Unsafe braking events.

- Inappropriate glance creating an unsafe condition.

- Merging or lane change errors.

- Inappropriate speed.

- Lane deviations occurring on straight roads.

- Tracking errors in turns or comers.

- Other cornering or turning errors (not tracking errors).

- Errors committed at intersections (not while making a turn). 
- Inappropriate stops due to use of a navigational aid.

- Inappropriate reaction to external events.

In addition, an "appropriate reaction to external events" category was created to measure the number of times drivers reacted appropriately to an unexpected external event.

In order to apply a "potential severity" number to a given near miss or driver error, events needed to be descriptively distinguished within the broad categories described above. A tool was developed to allow a rater to analyze the videotape of the events and consistently quantify the severity of safety-related errors. The tool, shown in appendix C, was developed in the form of a flow chart that guided the rater to a final description of the event circumstances and an associated error number. Accident data from the National Highway Traffic Safety Administrations General Estimates System were used to determine the different potential severity values within each event description.@ @ All of the four elements mentioned above that contribute to the potential severity of a safety-related driving error, were included in the final description. Close to 200 different combinations of elements were assessed by following the various paths of the flow charts. From this detailed description, three key safety risk factors were analyzed independently; "potential severity" of the event, "environmental proximity" of a hazard, and whether or not the navigational aid appeared to be a direct causal factor for the unsafe event. A listing of all the detailed error descriptions, along with the assigned potential severity values, based on speed were generated from the flow chart tool. These descriptions are shown in appendix D. Notice that the first number, designated with a \# symbol, is used for data grouping and reduction. These numbers do not necessarily follow in chronological order.

In determining the potential severity of an event, it was assumed that the worst-case accident could occur. Worst-case was defined as the most likely severe accident that would occur assuming a single change in pre-event circumstances. The potential severity, therefore, is a hypothetical event, created even if the elements to actually create an accident were not present. For example, consider the case where a driver was traveling at $89 \mathrm{~km} / \mathrm{h}$ $(55 \mathrm{mi} / \mathrm{h})$ on a two-lane road and deviated out of the lane boundary into the oncoming lane of traffic. The worst-case accident would be a head-on collision, given a hypothetical vehicle in the opposing lane were present. Whether the vehicle was present or not will be factored separately with the "environmental proximity" indicator to be discussed later. The potential severity of the example is high, because the approach speeds and striking angles would produce extreme deformation of the vehicle structure. Given other circumstances, such as lower speeds or angles other than head-on, the potential severity would be lower. The National Highway Traffic Safety Administrations General Estimates System helped to determine the potential severity values for these worse-case scenarios.@@

By analyzing the worst-case circumstances, four categories of severity ratings were created. These categories, described below, were developed based on operational definitions commonly used in system safety analysis. Note that in all determinations of potential severity, it was assumed that traffic was present (whether in reality it was or not), and that the worst reasonably feasible accident occurred.

\section{Operational Definitions}

\section{Operational D efinitions For Potential Severity Categories}

(4) Catastrophic - Potential for an accident where a fatality or permanent disabling injury is likely to occur (e.g., head-on collision or running a red light on a multilane road with a $72 \mathrm{~km} / \mathrm{h}(45 \mathrm{mi} / \mathrm{h})$ speed limit. 
(3) Critical- Potential for accident where injuries are severe enough to require overnight (or longer) hospitalization. The injuries, however, would not likely be permanently disabling. [e.g., running two-way stop signs in a residential area where the speed limit is $56 \mathrm{~km} / \mathrm{h}(35 \mathrm{mi} / \mathrm{h})]$.

(2) Marginal- Potential for an accident where injuries are feasible, but would not likely be severe enough to require hospitalization [e.g., side-swiping a car at $56 \mathrm{~km} / \mathrm{h}(35 \mathrm{mi} / \mathrm{h})$ because of a lane deviation].

(1) Minor - Potential for an accident occurring in which only property damage would likely occur [e.g., hitting a curb due to a lane deviation at $40 \mathrm{~km} / \mathrm{h}(25 \mathrm{mi} / \mathrm{h})$ and damaging a rim, but not causing physical harm to the driver].

Because there were no actual accidents, the severity number represents a worst-case scenario that did not occur, and provides no examination of the degree to which the error was a "close call." For example, previous research has shown that drivers using a navigation system have fewer lane deviations when other traffic is present.(22) Therefore, drivers may have been more accepting of the commission of error in circumstances that were not inherently unsafe (e.g., a minor lane deviation may not be inherently unsafe if no traffic is present).

To address this issue, events were also classified by the "environmental proximity" of a hazard to the camera car. The environmental proximity for each error was placed into one of three categories; near miss, hazard present, and no hazard present. The operational definitions of these categories can be found below. If an event occurred where there was in fact no hazard present, then the resulting criticality of the event was viewed appropriately in this context.

\section{Operational Definitions For Environmental Proximity Categories}

Near Miss. The driver is startled by a situation and is required to take immediate evasive action in order to prevent an accident. Near misses include situations where experimenters had to give an imperative verbal warning to drivers in order to bring their attention to unsafe situations. An example of this might be when the experimenter needs to call out "Red light!" because it appears that the driver will proceed through it if not told. Even though the subject may stop in time, and not actually enter the intersection, the startle response or experimenter intervention was a primary cause for the avoidance of danger.

Hazard Present. The driver commits a safety-related error when an object (e.g., another vehicle, a pedestrian, or a guardrail) is present in the environment. "Hazard present" requires that the object is in a close enough proximity to represent a hazard to the test vehicle, but not close enough that an immediate evasive action must be taken to avoid it.

No Hazard Present. The driver commits a safety-related error, but no close-proximity obstacle is present in the environment. An example of this would be a lane deviation where there are no objects near the test vehicle that constitute a hazard. The lane deviation still needs to be considered a safety-related error, even though the camera car was in no immediate danger.

A final error classification addressed the issue of whether or not navigation conditions were a causal factor in the commission of the error. Determining each error involved carefully reviewing the videotape data and listening to the audio track. During the review process, the analyst watched (several times) where the subject was looking prior to the event and 
listened to determine if the TravTek voice system was speaking prior to the event. In addition, the subjects often provided an unsolicited commentary about the occurrence of the event, which aided in determining the causal factors. Utilizing these observations, the analysts made their best assessments as to whether or not the navigation condition was a causal factor. The operational definitions of this categorization appear below.

\section{O perational Definitions For Navigation Condition as a Causal Factor}

Navigation Condition Caused. This condition exists when the root cause of the safetyrelated error was heavily influenced by looking at the displayed navigational information or listening to the TravTek voice. This includes glances at the CRT information, paper map, or written directions. If the driver is looking forward and commits a safety-related error, and it is obviously caused by focusing attention on the TravTek voice then it is still quantified as being caused by navigation information.

All Glance Locations Considered. This condition exists when the root cause of the safetyrelated error was not specifically influenced by looking at the displayed navigational information or listening to the TravTek voice. Instead, it apparently occurred as part of the driver's normal operating behavior, which is likely to include a certain number of safetyrelated driving errors that are independent of navigational aid use.

\section{Measures of Performance for Safety}

In order to find the safety-related driving errors, several measures of performance were collected that served as "triggers" to view specific portions of the videotaped subject runs. The triggers occurred within the data stream collected during testing, and were either placed by the experimenter or were extreme values flagged in the computerized portion of data collection. All videotaped runs were also reviewed by a second rater for errors on the experimenters part. It should be noted that a "trigger" in and of itself, does not denote an unsafe circumstance; it is only a cue to closely analyze a situation. The above mentioned flow chart tool was used to determine whether an event was safety-related, and to what severity level. Ultimately, the measures of performance serve only as criteria for determining where to conduct detailed video analysis. The measures of performance that were collected for this purpose are briefly described in the following paragraphs.

\section{Number of Accidents}

As mentioned before, no accidents occurred.

\section{Number of Near Misses}

A count of near miss occurrences, as defined above, was conducted in order to observe differences in frequency for the various display configurations. Near misses are considered the best estimate of future potential accidents.

\section{Single Eye Glances Greater Than $2.5 \mathrm{~s}$}

Glance duration was recorded and the data were reduced in such a way that each driver glance to the nearest $0.1 \mathrm{~S}$ could be identified. Lengths of single glances to the display (map) configuration are of particular interest to system safety. Bhise, Forbes, and Farber have suggested that, based on speed and travel distances, any single display glance greater than $2.5 \mathrm{~S}$ is inherently dangerous.(18) Based on this research, $2.5 \mathrm{~S}$ was used as a criterion to assess instances of unsafe behavior. Potential hazard environmental proximity was a 
key element in assessing whether the long glance was a safety-related event or not. If no hazard was present, then a long glance alone was not considered a safety error or event.

\section{Location of Glances}

When using navigation systems, drivers tended to glance back and forth between the display and the forward roadway. Distinguishing safety-related errors that occurred as a direct cause of navigational display glances helped identify which configurations required high visual attention. However, this measure of performance, in and of itself, was not a safety issue. Therefore, no results were tabulated based solely on one or more glances to a location. The use of the criterion "navigational aid as causal factor" (described above), distinguishes between errors caused by attention focused on either the display or the roadway.

\section{Abrupt Lateral Accelerations and Braking Maneuvers}

The camera car automatically recorded all lateral and longitudinal accelerations. A value of $3.9 \mathrm{~m} / \mathrm{s} 2(0.40 \mathrm{~g})$ was set as a trigger point for further analysis of excessive acceleration (lateral or longitudinal) to determine if an unsafe event occurred. Late braking reactions that occurred due to conditions in the driving environment such as slow traffic or traffic control devices were also marked in the data stream by the experimenter.

\section{Unplanned Lane Deviations}

All lane deviations were classified and timed by using a lane-track camera view. An unplanned lane deviation is a face valid indicator of driver inattention and accident potential. It is important to distinguish between lane deviations caused directly by navigation condition attention and deviations caused by other factors. Other factors that could cause lane deviations include; unsafe driving habits such as clipping corners, poor roadway design or poorly painted lines, and traffic situations that force a lane deviation. Lane deviations that occur while attention is focused to the navigational aid, are a better estimate of the workload required by that navigation condition.

\section{Reaction to External Events}

When driving, occasionally there is a maneuver required to avoid some unpredictable hazard in the roadway ahead of the vehicle (e.g., another vehicle pulled out suddenly in front of the driver or there was debris in the roadway). Analysis of the reaction to these external events is indicative of the degree to which the driver's attention was diverted to a given navigation condition. Inappropriate reactions are considered unsafe events and are good indicators of overload. Appropriate reactions also provide a counter indicator, and although no safety critical event occurred, one was essentially avoided. Therefore, both appropriate and inappropriate reactions to external events were considered in the safety analysis.

\section{Inappropriate Speed}

Slow speeds due to glances at the display configuration indicated driver inattention to the driving task. Variation in speed increased the hazard potential due to changes in closing rates between the camera car and proximal traffic. In determining accident potential, special attention was placed on where the glance location was predominately focused when speeds were extremely slow. 


\section{Stopping in Unsafe Circumstances}

Stopping in a location that created potential for an accident was broken down into two different types of hazards. The first consisted of the driver slowing to a stop in order to retrieve navigation information, but choosing an unsafe location in which to stop (e.g., the driver pulled off on the shoulder of a busy Interstate roadway, partially off on the shoulder of a two-lane roadway, or stopped in a lane of traffic). The second hazard consisted of the driver making a normal intersection stop for a traffic control device, but sitting for a long period of time collecting navigation information instead of proceeding through the intersection in a correct and legal fashion. Note that when camera car drivers remained stopped at a green traffic light, other drivers were confused about their intentions.

\section{Subjective Workload Ratings for O verload}

These ratings give a good indication of workload situations that may not necessarily cause a performance decrement, but still cause high attention demand. If the subject rated all three dimensions of the subjective workload scale as high, these cases were considered to be a relevant safety event due to the increased stress level. A comparison of these high workload ratings was conducted across the different display configurations.

\section{Dangerously Close Headways}

When drivers followed at close headways, the attention required to effectively avoid accidents increased greatly. Frequent or extended glances at the navigation information display rather than the forward roadway, constitute an increase in accident potential. Differences across display conditions was analyzed for these circumstances.

\section{Interpretation Of The Camera Car Safety Analysis Results}

In many ways the Camera Car Study served as a "stress test," encompassing many of the worst-case aspects of workload that are related to safety. As mentioned before, navigating to an unfamiliar destination was a difficult task. Factors such as extremes in age, the presence of an experimenter, and unfamiliarity with the vehicle increased the task difficulty more than it would have in normal non-navigation use. In addition, the potential severity ratings were judged based on a worse-case scenario. The method of looking at the safetyrelated errors was accomplished using a conservative analyses that followed the experimental methodology used in industrial safety techniques.

Another factor to consider in the safety analysis, is that there were no accidents and only a few dozen true "near misses" that occurred in 252 data collection runs. These provide the most direct estimates of accident risk. Once the estimation of accident potential reached the level of analyzing "hazard present" and "no hazard present" events, the estimation (although valuable for comparing the safety of the different navigation conditions) lost some level of validity.

One final and important element to consider when interpreting these results, is the amount of ability and driving safety variability among drivers. Figures 44 and 45 show that there were a few drivers who had a disproportional number of errors in comparison to the average number of unsafe incidents. The difference for drivers who were in the visitor group (see figure 45), ranged from only 4 errors committed by the safest drivers to 48 errors for the worst driver. The same trend held true for the local users with only 18 errors committed by the safest drivers compared to 74 for the worst driver. Fortunately, each subject used all six navigation conditions, so reasonable comparisons across methods could be made. The trends of errors committed by subject number 7 (figure 44) and number 26 
(figure 45) followed the typical pattern of the combined subject data across the 6 navigation conditions. It would appear that their increased rate of error did not adversely effect the resulting conclusions or methodology used by the study. With all these considerations mentioned, it is possible to view the results of this safety analysis in proper context and assess its true meaning with respect to safety costs and benefits.

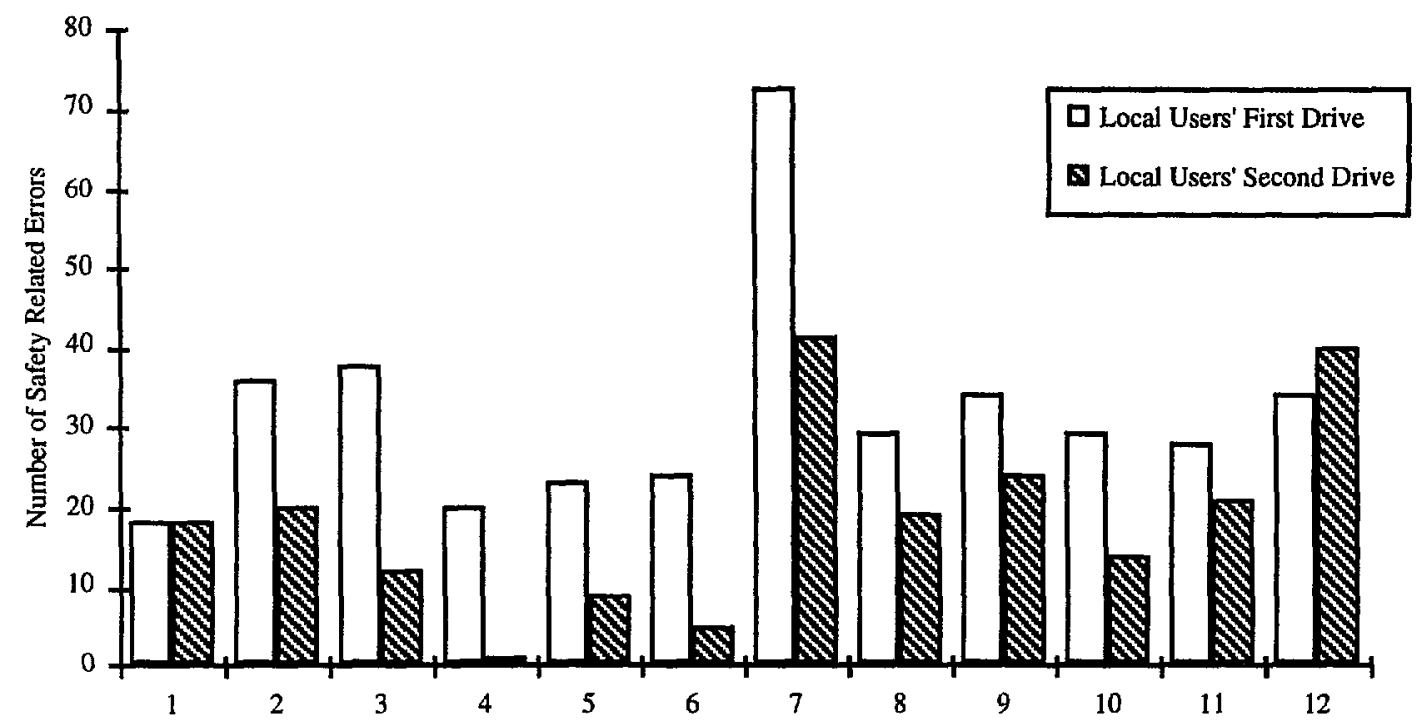

Figure 44. Total number of safety-related errors committed by local users; shown by individual subjects.

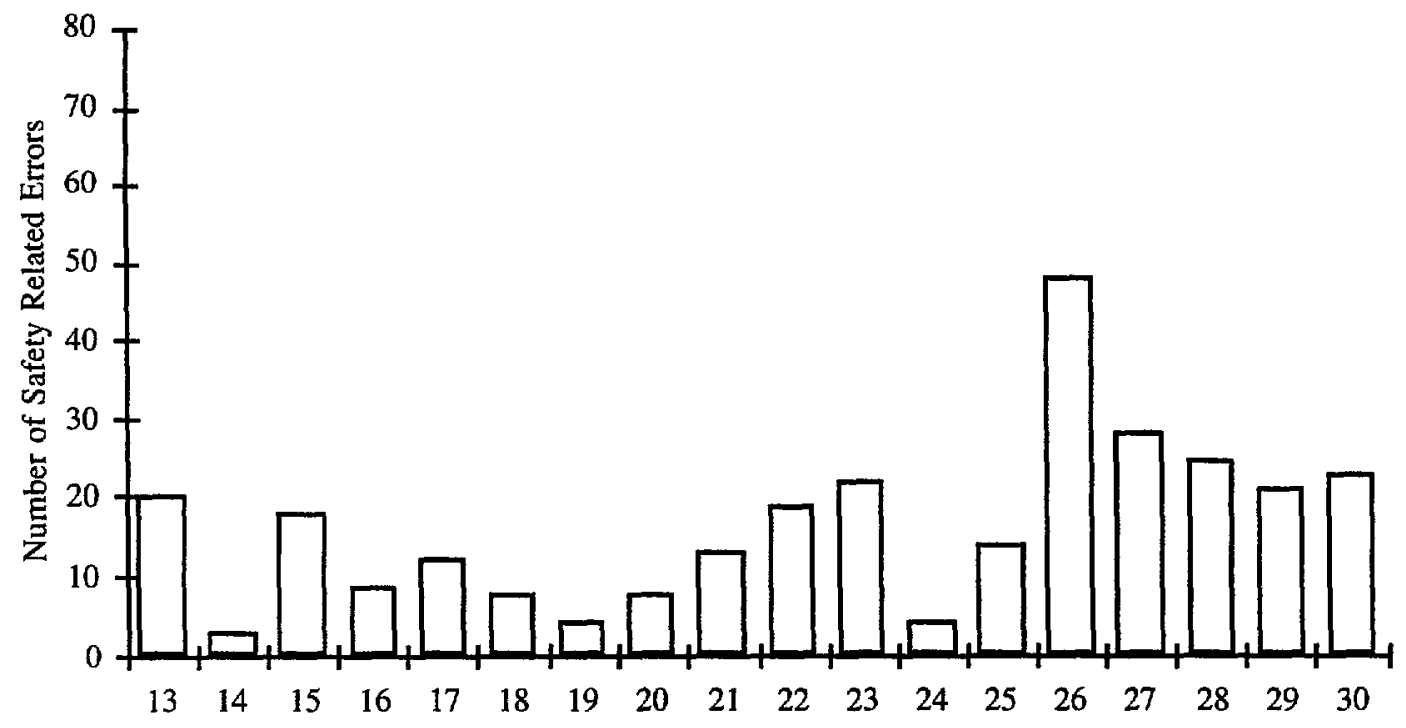

Figure 45. Total number of safety-related errors committed for visitors; shown by individual subjects. 


\section{Measures of Performance for Near Misses and Unsafe Acts}

The results of the TravTek navigation system safety analysis and the control conditions of paper map and paper directions have been divided into several individual performance measures. Each of these measures are discussed in the following paragraphs. Unlike many other performance measures in this report, the safety analysis did not use tests of statistical significance to determine differences between navigational conditions. In many cases, the number of observations were such that there were missing cell values due to the method of observing naturally occurring events. In most other cases, a large number of statistical comparisons would have been required to interpret the data. Since the data are nominal, parametric post-hoc comparison tests, which have the advantage of controlling type I errors, were inappropriate. Thus, non-parametric, post-hoc comparisons were required without type I error protection, requiring a reduction in the alpha criterion-level. The increase in type II errors that would have occurred under these circumstances took away the ability to detect differences between the displays. Comparisons of frequency were used to solve these issues. The reader is advised to interpret specific differences in the data with caution, especially when the number of occurrences of data division is quite small.

\section{Number of Accidents}

No accidents occurred during the Camera Car Study.

\section{Number of Near Misses, Plus 0 ther M easures of Environmental Proximity}

When considering all glance locations, the turn-by-turn without voice display had only four (the lowest number) near-miss errors across all the user groups. The paper map control condition had seven near misses. Based on near misses, the least safe navigational condition was the route map (with or without voice). There were a total of 21 and 23 (respectively) near-miss incidents within the route map condition. The paper direction condition had 11 near-miss errors, while the turn-by-turn with voice condition had 10 errors. The number of near misses that occurred for all glance locations is shown in figure 46.

Errors caused by attention directed at the navigational aid also need to be considered. However, there are fewer numbers on which to base an assumption of display condition differences. When only these errors are considered, turn-by-turn without voice had no near misses. Similarly, the paper map condition had only one near miss; turn-by-turn with voice, route map with voice, and paper directions each had three near-miss errors, and route map without voice had five near-miss errors. The number of near-miss errors that occurred while attention was focused on the navigational aid is shown in figure 47.

While near misses are the.best measure available for accident estimation, there are relatively few near misses on which to base navigation condition comparisons. Analyzing the next two levels of environmental proximity will perhaps aid in distinguishing between the relative safety of using the various navigational conditions. Figure 46 shows the rates of occurrence for "hazard present" and "no hazard present" driver errors when considering all glance locations. The next best safety predictor, is errors committed in the presence of a hazard. The paper map condition had the lowest number with 50 hazard-present errors, followed by the paper directions with 54 errors, and the turn-by-turn with voice with 57 errors. The route map without voice condition had a substantially higher number, with 138 errors. 
The paper map condition had the lowest number of hazard present driving errors in relation to navigation condition attention (see figure 47 ). This was followed by the turn-by-turn with voice and paper direction conditions, with 31 and 32 respectively. The route map without voice had the most hazard present errors with 106 . Note that overall, there were at least twice the number of route map without voice errors as any other display configuration and almost six times the number of errors as the paper map condition.

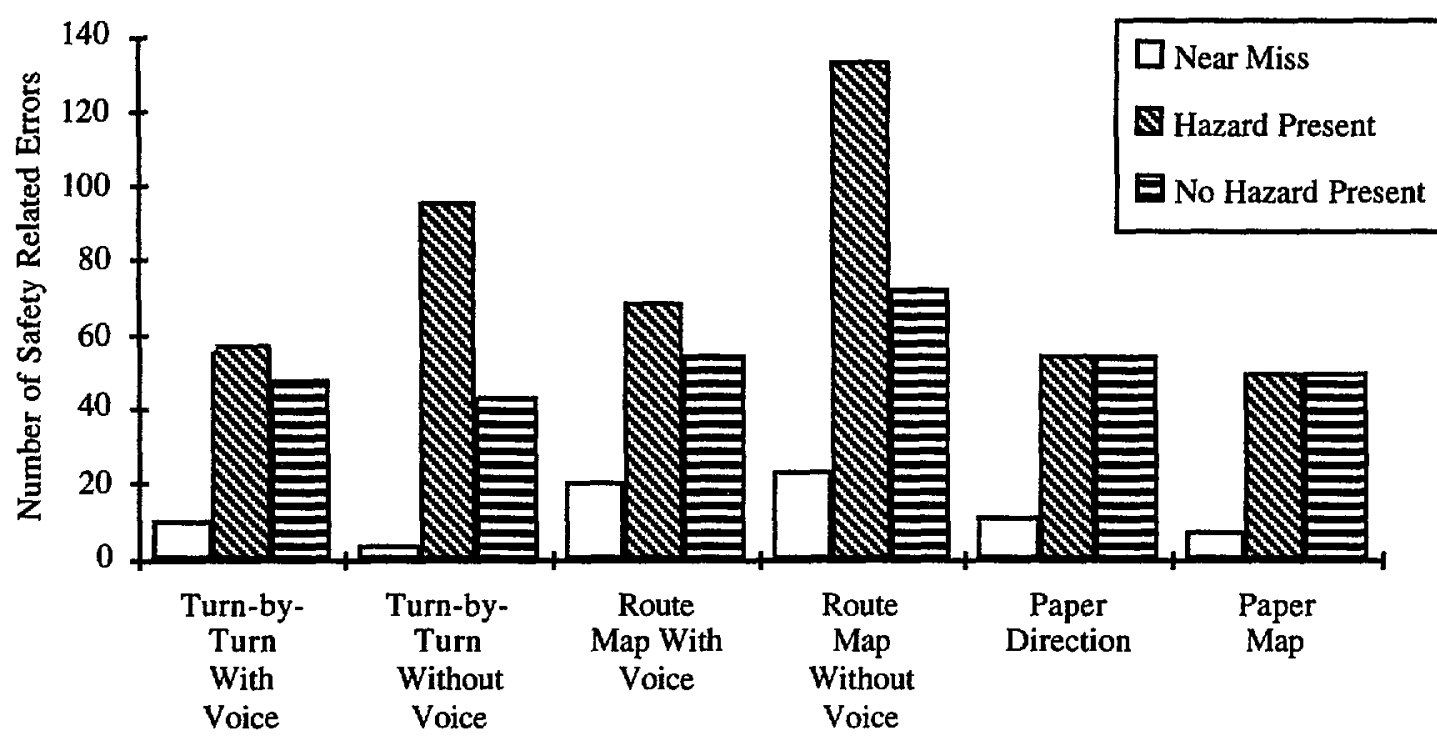

Figure 46. Number of safety-related errors for each navigation condition and each environmental proximity; all data included.

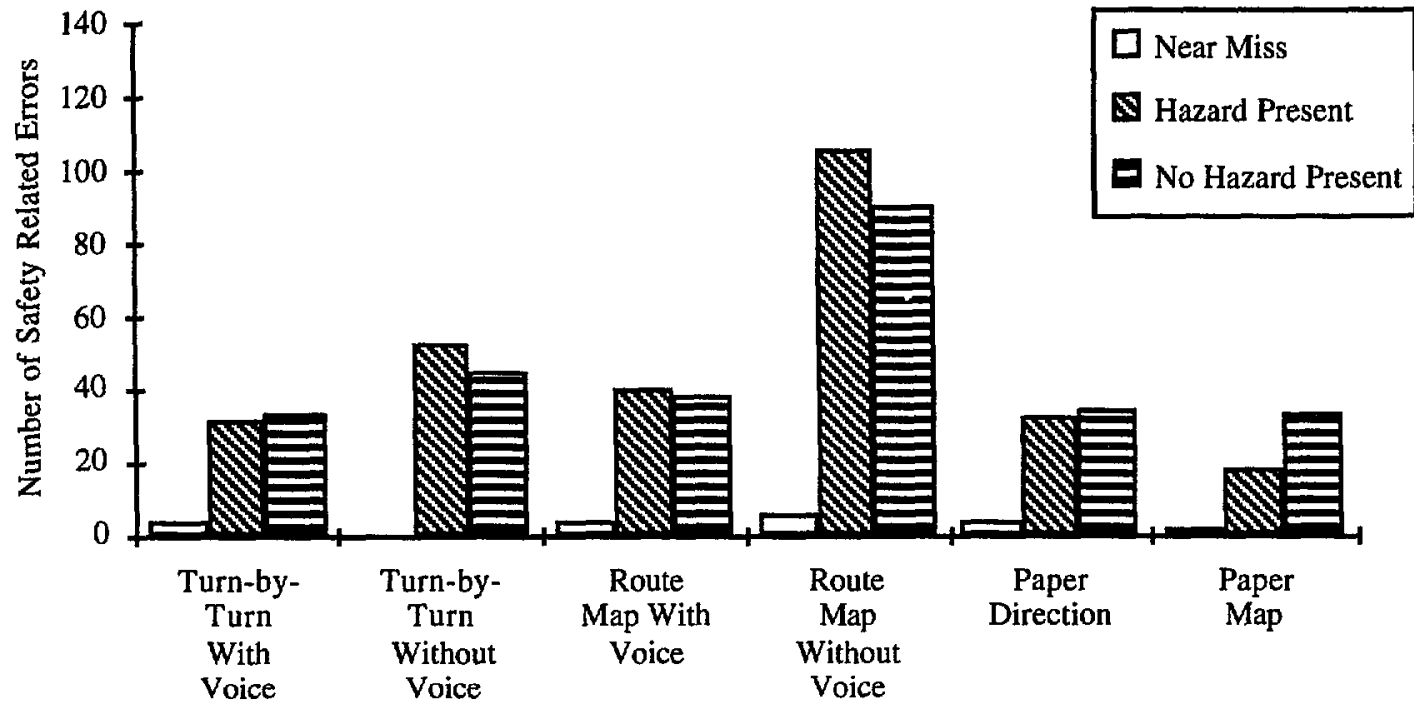

Figure 47. Number of safety-related errors caused by a glance at the navigation display for each navigation condition and each environmental proximity; all data included. 
When considering all the safety-related errors that occurred when no hazard was present in the environment, the trend across the display conditions was relatively unchanged. In fact, there are almost an identical number of errors in the hazard present classification. Figures 46 and 47 show the details of the results; since the numbers and trends are so similar, further distinctions have not been made.

\section{Number and Potential Severity of Incidents}

The potential severity value is useful overall, for comparing the relative severity of the errors. However, this value was assigned independent of the presence of a true near miss or the proximity of a hazard. Figure 48 shows the number of incidents of each severity type for the various navigation conditions. Note that the potential severity value is only a worst-case scenario estimate of an unsafe condition.

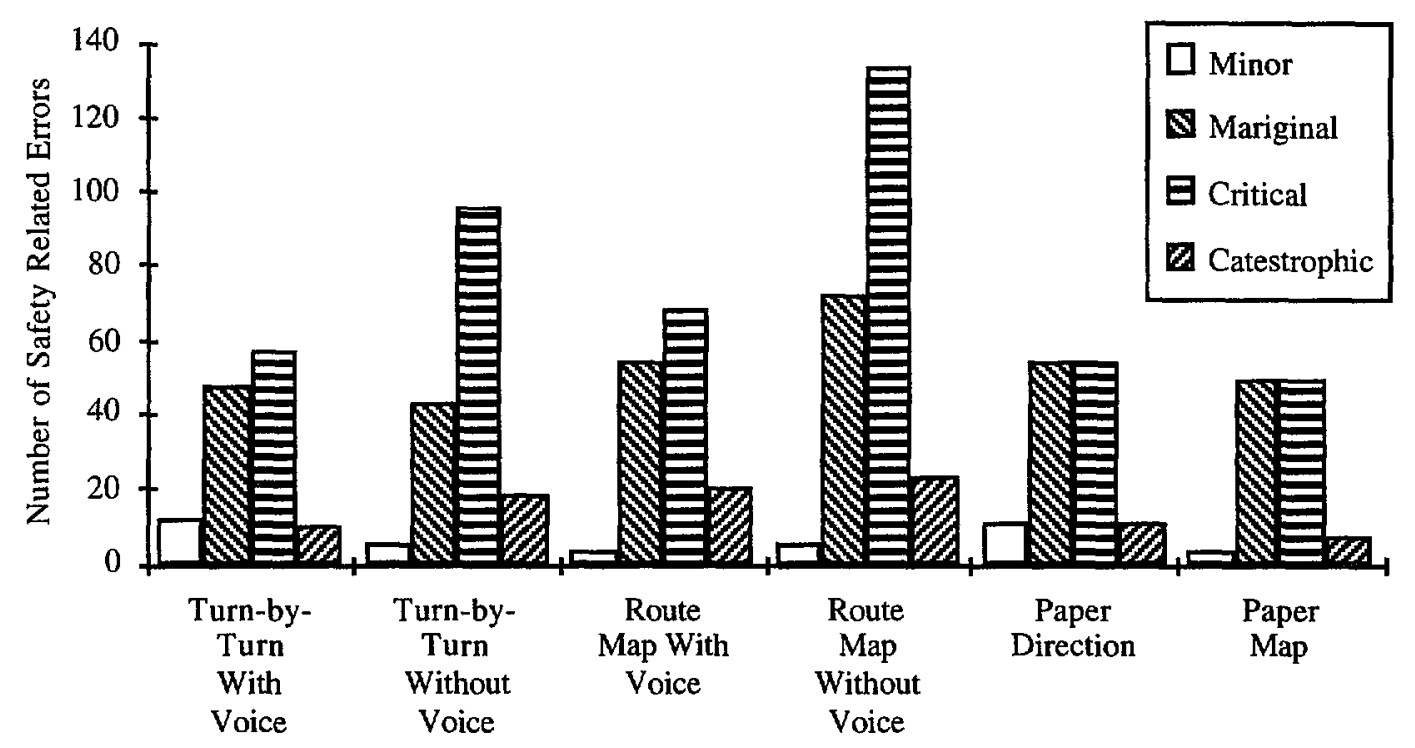

Figure 48. Number of safety-related errors for each navigation condition and each potential severity category; all data included.

The route map conditions had the most "catastrophic" potential severity errors with 23 instances for the route map condition without voice, and 21 instances in the route map with voice condition (see figure 48). Turn-by-turn without voice had similar numbers of potential catastrophic events with 19 total. Turn-by-turn with voice and paper directions had lower numbers of incidents with 10 and 11 respectively. The least number of catastrophic incidents occurred in the paper map condition with seven events total. There was no meaningful distinction for catastrophic events between turn-by-turn with voice, paper directions, and paper maps; or between turn-by-turn without voice, route map with voice, and route map without voice. It should be noted that there were relatively few total occurrences of catastrophic events in comparison to critical and marginal potential severity values. This is a good indication that drivers were avoiding major potential mistakes on roads with high speed limits, busy intersections, or areas with danger of head-on collisions.

The number of critical severity safety-related errors for the potential severity category shown in figure 48 varies substantially between conditions. Route map without voice had 
the most occurrences with 134. This was followed by the turn-by-turn without voicecondition with 96. Route map with voice had the third highest number with 69 safety-relatederrors. Again, there was little distinction between turn-by-turn with voice, paper directions and paper map conditions with 57,54,50 errors respectively. Figure 48 shows that there was less distinction between the marginal and minor severity levels. However, the route map without voice had the highest number of critical andmarginal occurrences. To get an overall perspective of the differences between navigation-induced errors where a hazard and some degree of injury potentialwas present, an additional analysis wasconducted. This analysis did not consider the "no hazard present" category ofenvironmental proximity and the minor category of "potential severity". Figure 49 shows the total number of incidents across all error category types for the remaining data.

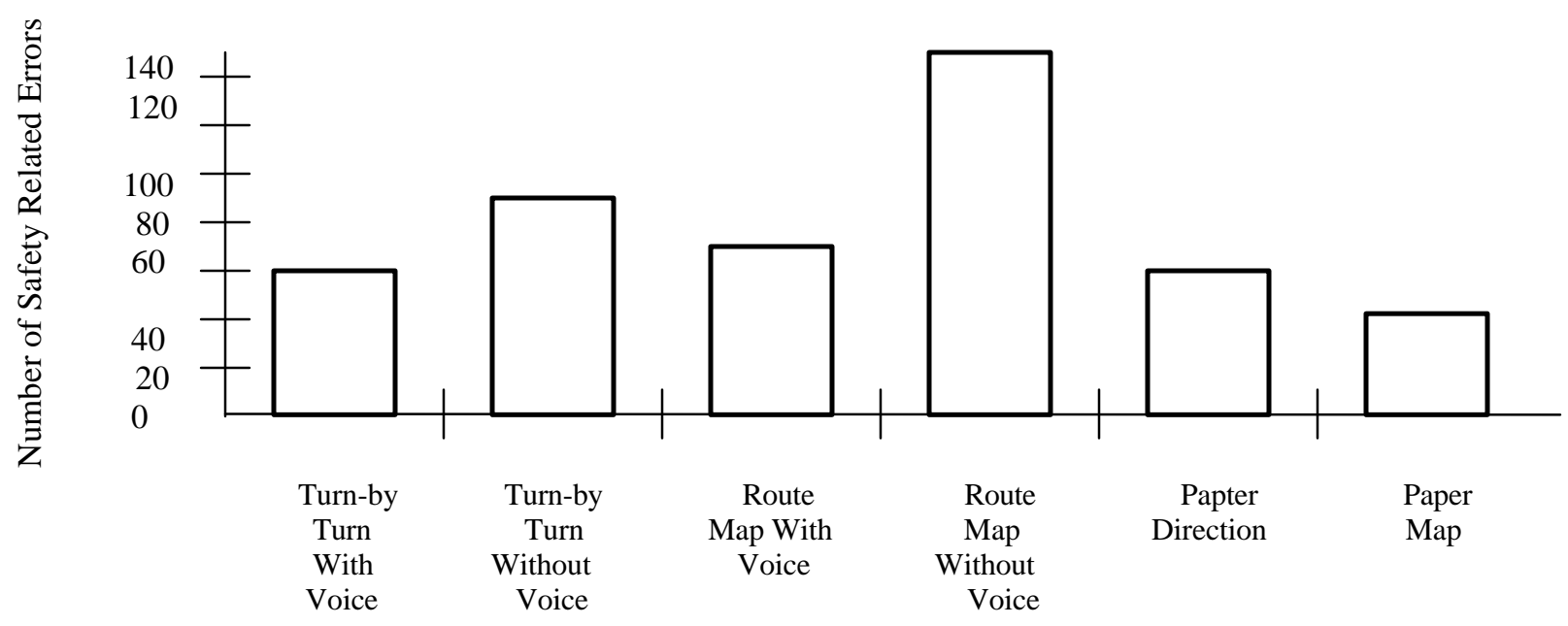

Figure 49. Number of safety-related errors for each navigation condition; no hazard present and minor severity incidents excluded.

Figure 49 shows that the route map without voice had 132 errors, the greatest number of incidents of unsafe driving errors among navigation conditions. The next largest numberof occurrences was turn-byturn without voice with 85 safety errors. This indicated that by adding the voice to a display, the number of safety-related driving errors was reduced. Route map with voice had a total of 66 errors, one-half of the same visual display withoutthe voice feature supplementing the information presentation. Note that the two TravTek voice conditions and the paper direction list condition had approximately 60 errors, while the paper map condition had the lowest number with approximately 40.

Another analysis measured the frequency with which subjects made mistakes within each error category type. This was performed to aid in the estimation of accident potential andultimate criticality. Figures 50 and 51 show the number of occurrences for each displaycondition across all of the previously defined categories (see appendix B). Some category trends are discussed in detail below. 


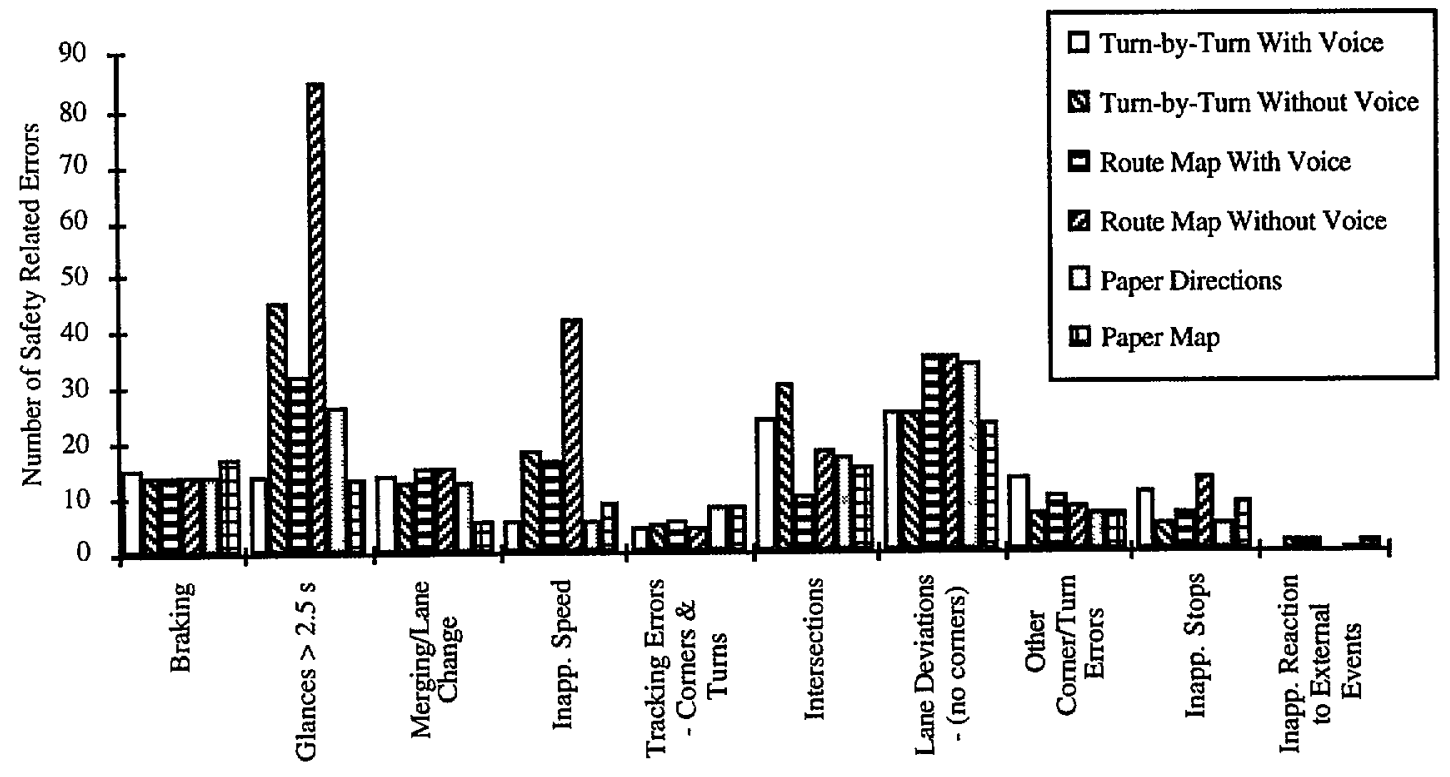

Figure 50. Number of incidents in each safety-related error category for each navigation condition; all data included.

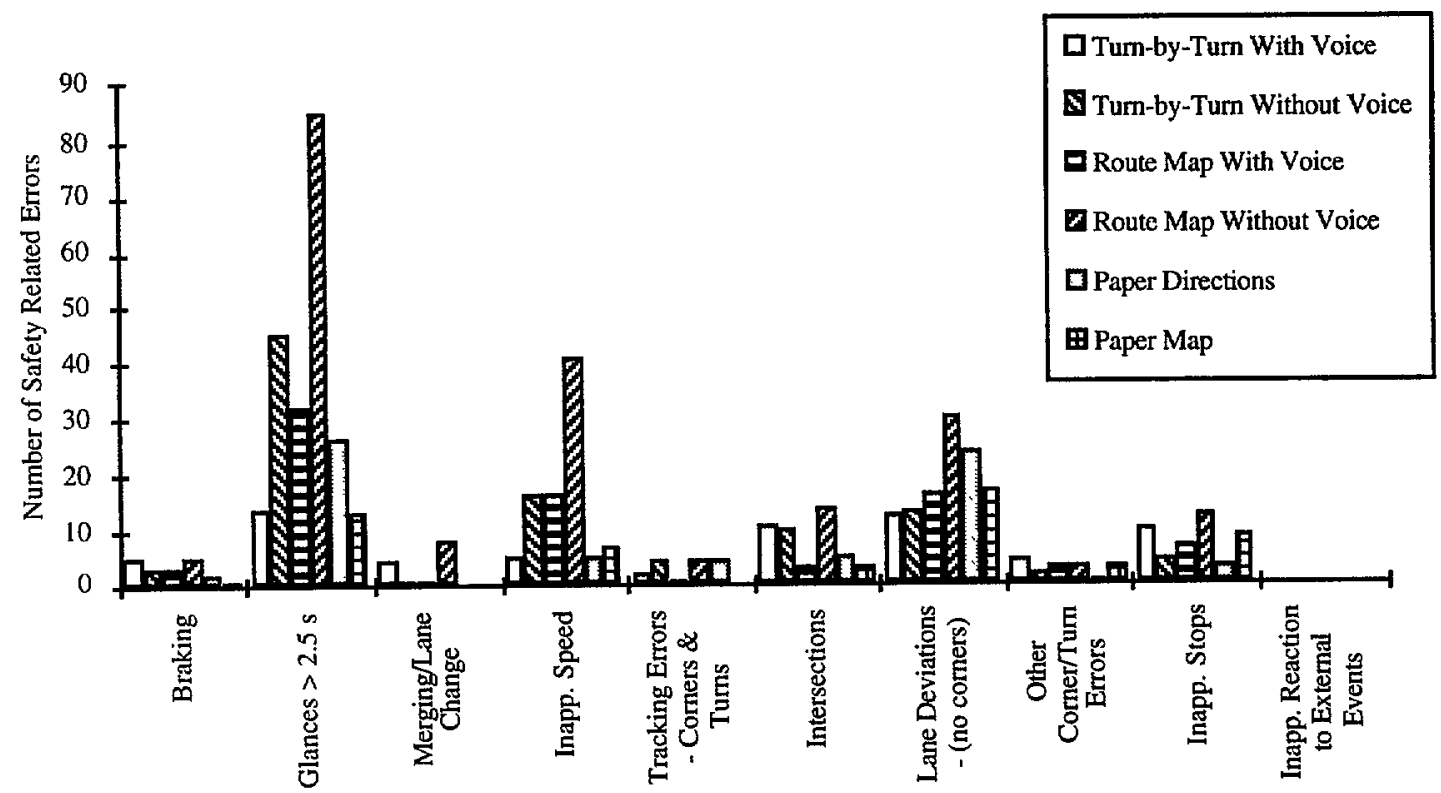

Figure 51. Number of incidents in each safety-related error category where attention was directed at the navigation display. Shown across each navigation condition.

Single Eye Glances Greater Than $2.5 \mathrm{~s}$

If subjects focused on the navigational aid for more than $2.5 \mathrm{~s}$ the occurrence was observed in order to determine if any safety-related errors were associated with the long glance. The differences in the number of safety-related incidents that occurred across display configurations are shown in the glances $>2.5 \mathrm{~s}$ portion of figure 51 . Instances where a glance over $2.5 \mathrm{~s}$ resulted in a safety-related error were highest for the route map without voice 
condition. This condition resulted in a total of 85 unsafe instances in which the long glanceocurred. This was a considerably higher than the 45 safety-related errors that occurred inthe turn-by-turn without voice condition. The lowest number of errors occurred in the paper map condition with only 13 errors, followed by turn-by-turn with voice with 14 errors. With regard to this safety measure, it appears that the turn-byturn with voice and paper map were equivalent. The route map with voice and paper directions also seem comparable. One would hypothesize, that a low number of safety-related errors occurred for navigation conditions requiring the least amount of visual attention.

\section{Abrupt Lateral Accelerations}

A value of $3.92 \mathrm{~m} / \mathrm{s} 2(0.40 \mathrm{~g})$ was set as a trigger point for detailed analysis of lateralacceleration to determine if an unsafe event occurred. The majority of these triggers werenot in fact safety-related events, but rather just intentional corners that exceeded the trigger point. Most of the "false alarms" were situations where the driver was entering a freeway on-ramp from an adjoining secondary road. Since no traffic was present, these were not considered safety critical events, which left few eventsin which to make display judgments. An increased number of false alarms given by this trigger assures that truly unsafe events were not missed in the data analysis.

The remaining events, which were deemed unsafe lateral accelerations, are shown in figure 52; this figure includes all user groups. Turn-by-turn with voice had the largest number ofunsafe maneuvers with a total of 9 . However, turn-by-turn without voice had the fewest unsafe lateral accelerations with only 2 incidents. The remaining navigation conditions had 4 or 5 occurrences. The low number of events made distinctions between displays difficult, especially since the two extremes had the same visual information presented to the driver.

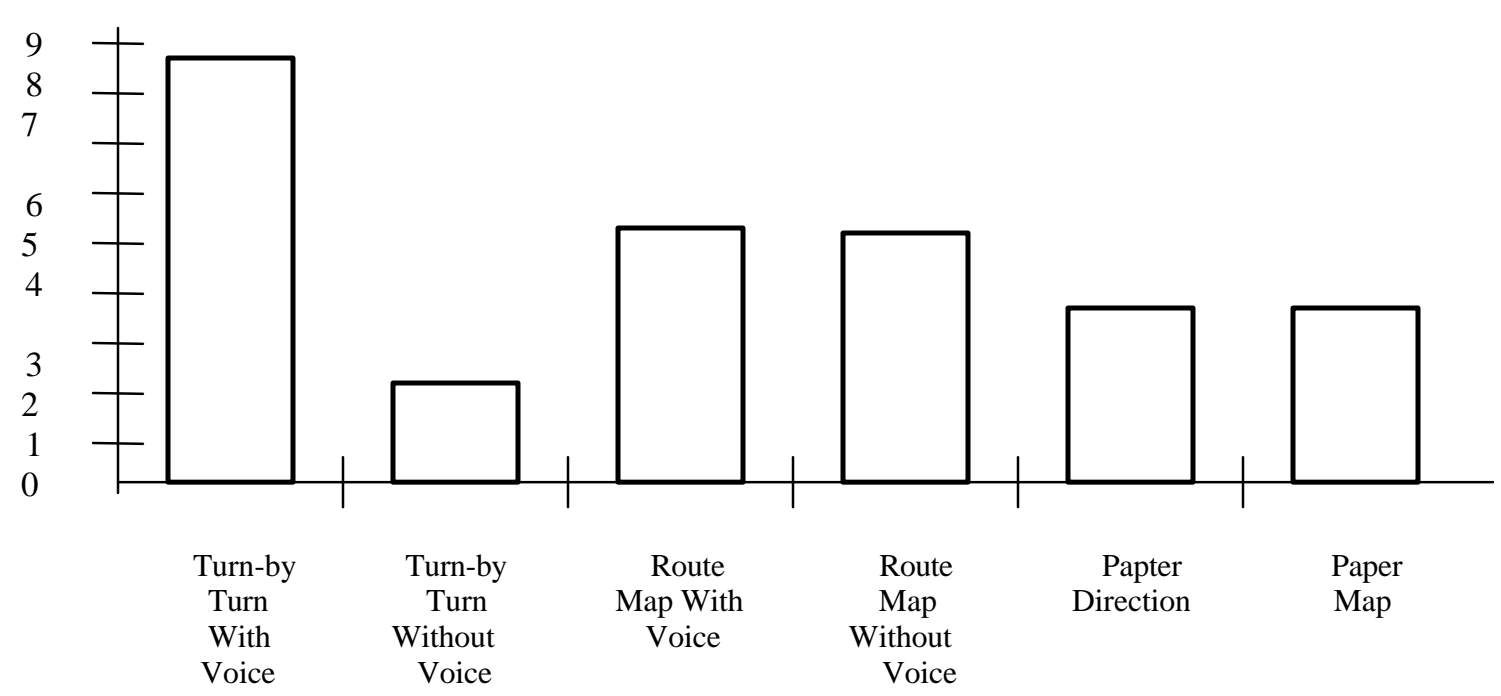

Figure 52. Number of safety-related errors that exceed designated safe lateral acceleration rate for each navigation condition; all data included. 


\section{Abrupt Braking Maneuvers}

Late braking reactions sometimes occurred when the driver was not paying attention tostopped/slow traffic or traffic control devices which indicated a need to stop. A display thatrequired a high degree of visual attention tended to cause these types of safety-related events to occur more frequently. Figure 53 shows the number of incidents of abruptbraking that occurred for the different navigationconfigurations. There are only a few incidents on which to base an analysis of the difference in accident potential, so the reader is advised to interpret with caution. Turn-by-turn with voice was low in unsafedecelerations, as well as the paper direction list and paper map condition. This indicatesthat the other displays; turn-byturn without voice, route map with voice, and route map without voice may have distracted the driver and resulted in more occurrences of late braking due to inattention.

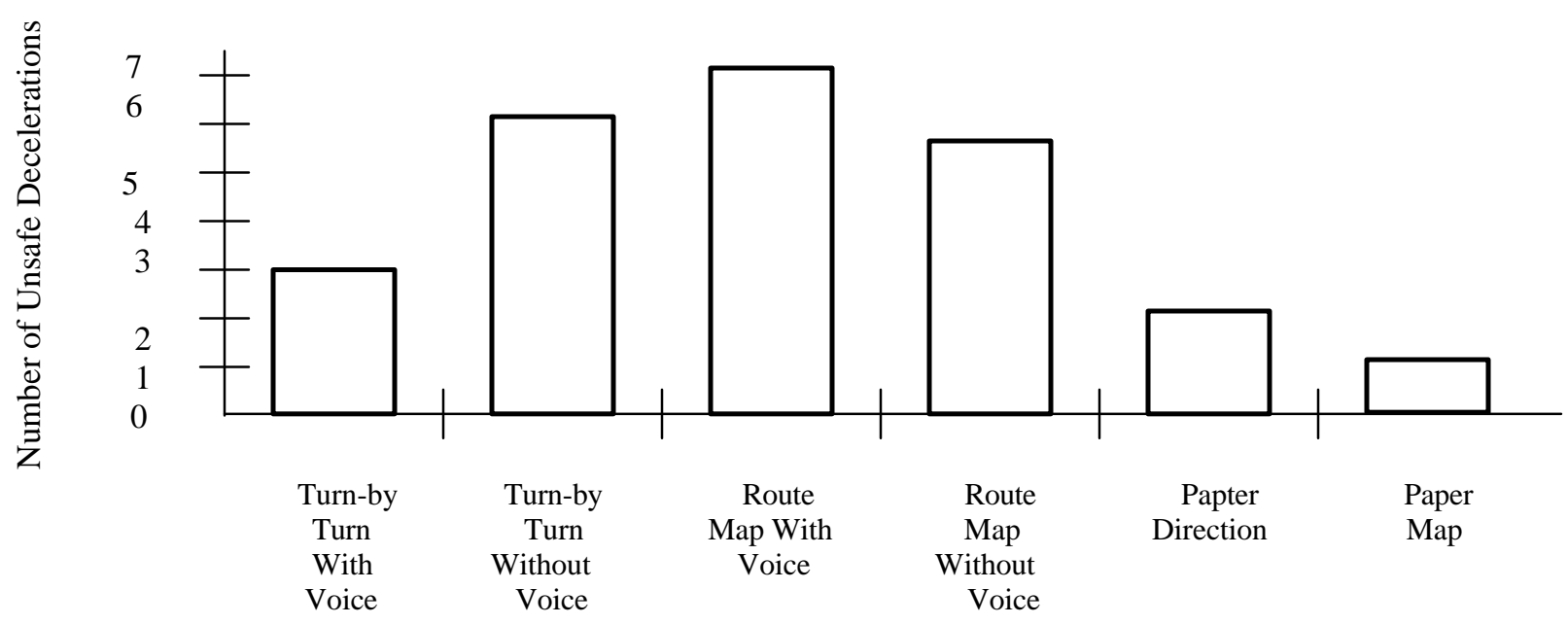

Figure 53. Number of safety-related errors that involved braking rates which exceed designated safe deceleration rates for each navigation condition.

\section{Unplanned Lane Deviations}

The overall average duration for a lane deviation during the Camera Car Study was 3.86 s. There was no significant difference between lane deviation duration for the navigationonditions.

While there was not a distinction between displaydurations, the number of unplanned lanedeviations was a face-valid indicator of driver inattention and accident potential. Consider-ableweight can be placed on the findings where the glance location was directed at thenavigation aid and an unplanned lane deviation occurred. Figure 54 shows the number of incidents of lane deviations for all occurrences, as well as those instances where attention was focused on the navigation aids. As shown, about one half of all the lane deviations were caused by the driver focusing attention on the navigation aid. However, this is nottrue for route map without voice. Out of 39 total lane deviations, 34 were caused (at least in part), by attention focused on the navigation display. Turn-by-turn with voice had thelowest number of lane deviations with 29 total deviations, and with 14 caused by attention 
focused on the display. There were 17 cases in each navigational condition for turn-byturn without voice, route map with voice, and paper map where glances at the navigational aid was attributed to lane deviations. There were 28 occurrences attributed to paper direction use.

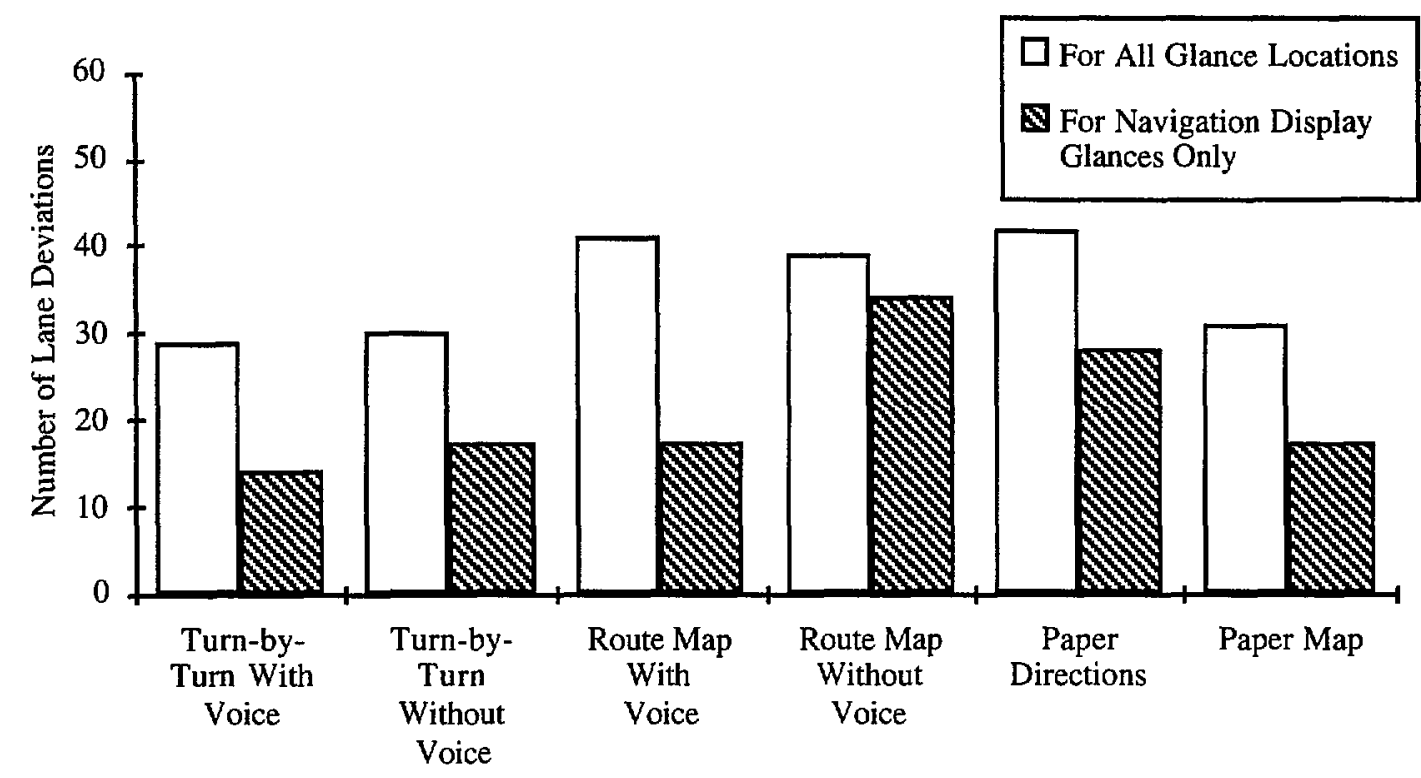

Figure 54. Total number of lane deviations for each navigation condition; shown for all glance locations and for deviations caused by a glance to the navigation display.

\section{Reaction to External Events}

A driver's reaction to external events was another measure of visual attention required by a navigation condition. This measure indicated circumstances where attention was shifted away from the driving task. Inappropriate reactions were considered unsafe events and were good indicators of overload; however, none occurred as a result of attention being focused on the navigation aid (see previous figure 51). When all instances were considered, as shown in figure 50, there were 7 total unsafe reactions to external events. Because of the low number, it is difficult to draw a conclusion comparing navigation conditions. The most important result was that no inappropriate reactions occurred because of a glance at any navigational device.

Figure 55 shows that there were more appropriate than inappropriate reactions to external events. Appropriate and inappropriate reactions provided a good indicator of the driver's ability to adapt to the demands of the system while responding to circumstances of unanticipated attention demand. If one condition required more visual attention than another (as seen in the driving task intrusion section), the number of glances increased more than the duration of glances. The subjects used their own judgment when considering the potential for an unexpected event, and when a reaction was required 34 out of 41 were appropriate. Because none of the inappropriate reactions occurred while switching attention between the road and the navigation device, it can be assumed that displays were used safely when unexpected circumstances arose. 


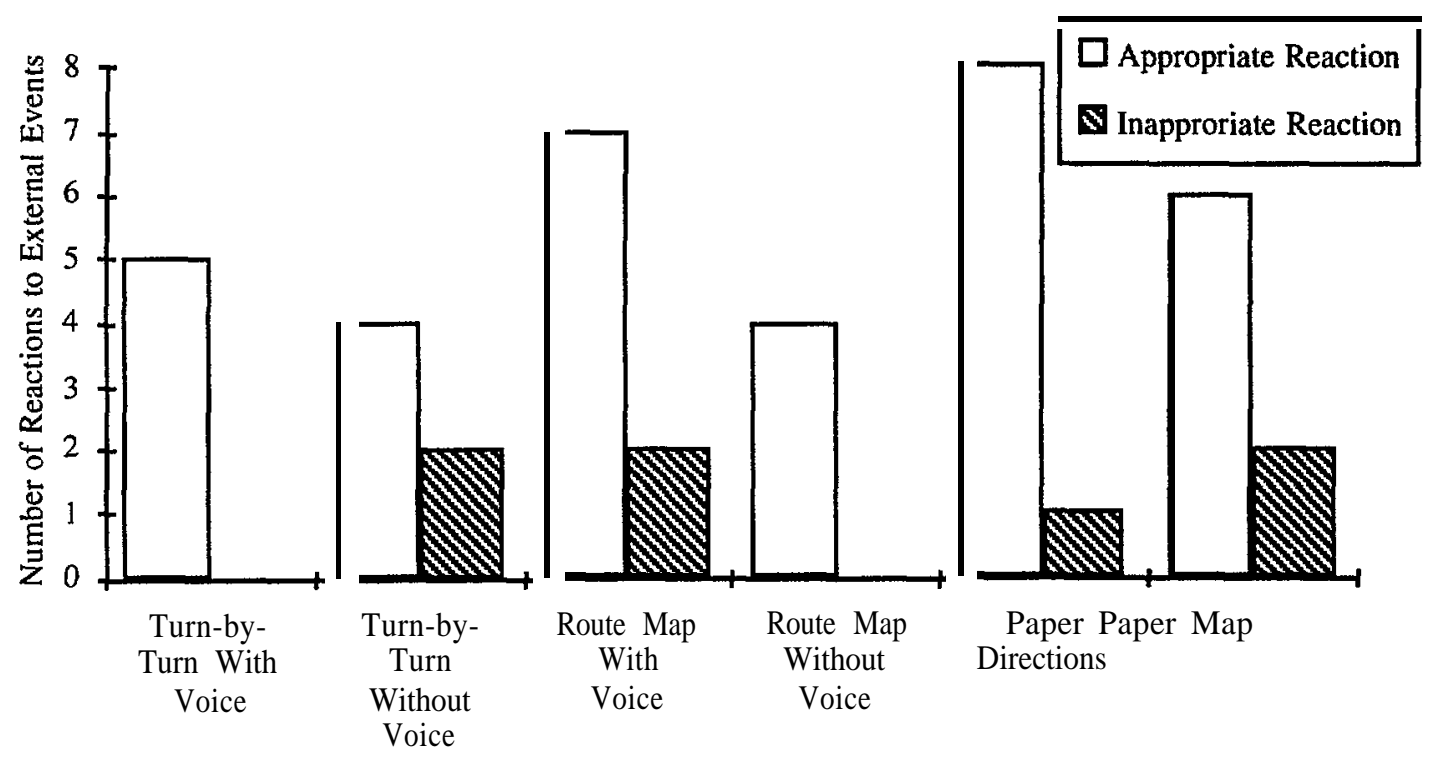

Figure 55. Total number of incidents which involved appropriate and inappropriate reaction to external events; shown for each navigation condition.

\section{Inappropriate Speed}

Retrieving navigation information that requires a high degree of attention has been shown to lead to circumstances where the driver slows inadvertently to compensate for the overload. There were 97 speed category errors (see figure 50) of which 94 were slowspeed related. Figure 56 shows the distribution of the slow speed errors across the different navigation conditions. The most occurrences of slow speed errors appeared in the route map without voice condition.

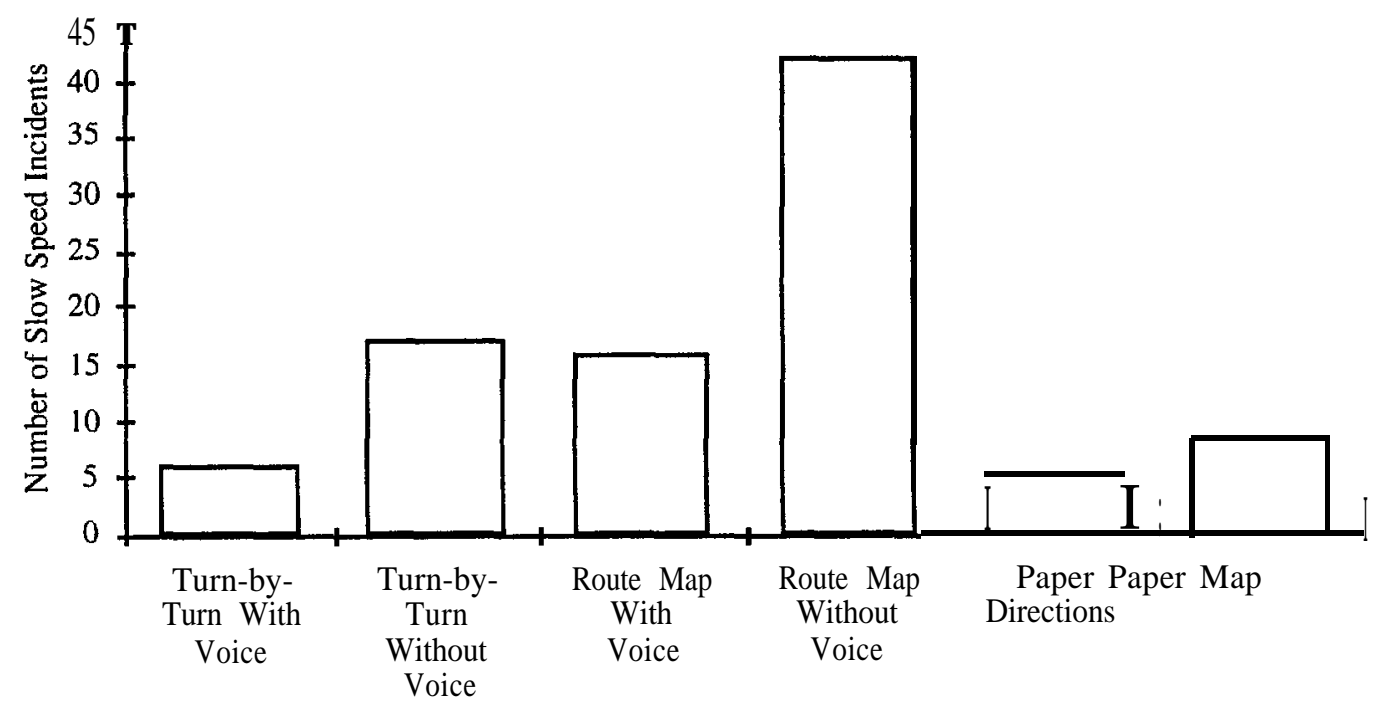

Figure 56. Total number of slow speed incidents committed within each navigation condition. 
Turn-by-turn without voice and route map with voice were comparable with 17 and 16incidents respectively. The paper map condition had eight errors. The turn-by-turn withvoice and the paper directions had the lowest with six and five respectively. Theimplication of slow speed on accident potential varied greatly with the road type, relative speeds of traffic, and the degree to which the camera car slowed.

\section{Stopped Vehicle in Unsafe Circumstances}

There were two circumstances where stopping to collect navigation information presented ahazard to the camera car and driver; pulling to a stop in an unsafe location and a driverstopping at an intersection for an inappropriately long period of time while collectingnavigation information. Figure 57 shows the incidents of unsafe stopping situations. Thenumber of instances was low, and differences are not likely to be reliable, but the incidentswere evenly distributed among subjects. It has been hypothesized that providing a design that "locks-out functions" while the vehicle is moving, will have a safety benefit of reduced attention; however, it will have a safety cost of forcing (or enticing) the driver to pull-overin unsafe circumstances. These data clearly refutes the safety cost portion of the hypothesiswith regard to unsafe stops in roadways (but not at intersections). Only 13 times in 252data collection runs, did drivers pull over in unsafe circumstances, 4 of which occurred inthe control conditions.

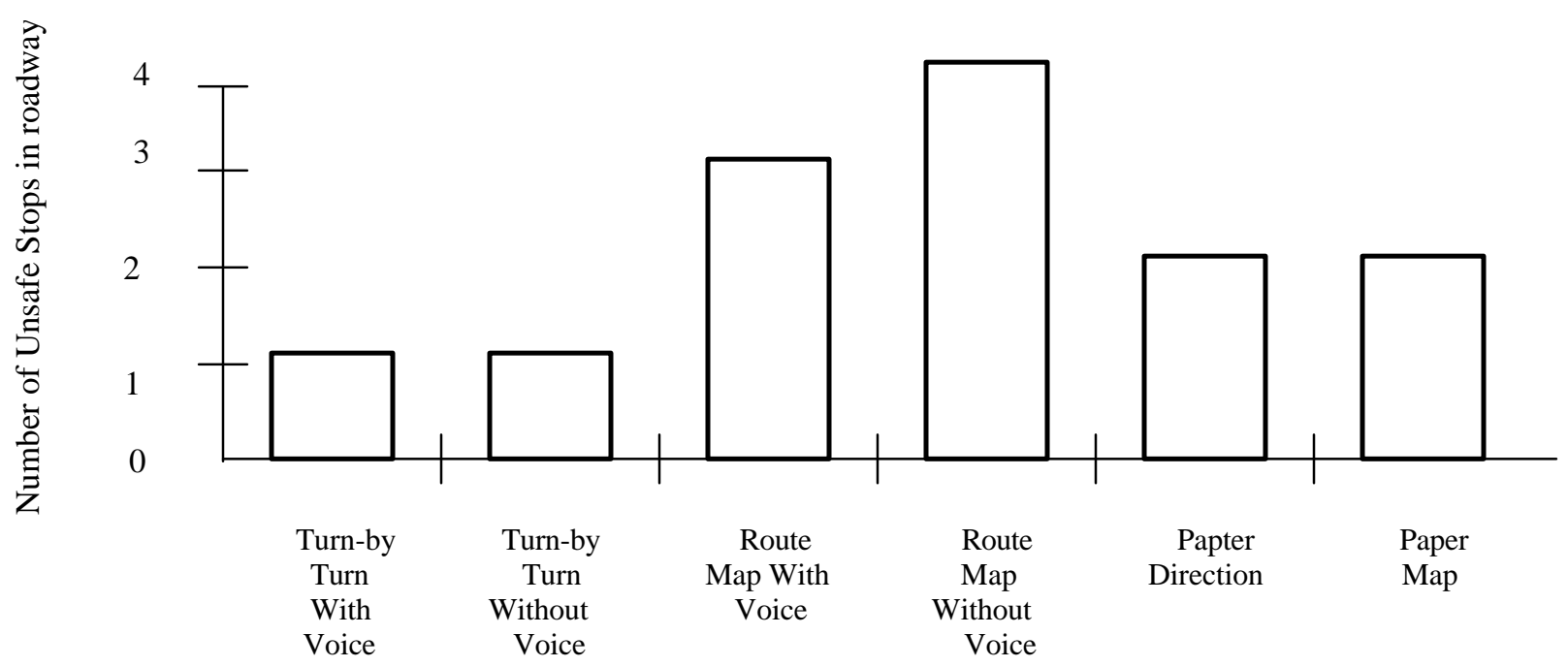

Figure 57. Total number of stops in the roadway or other unsafe location where the user needed to gather navigation information. Shown for each navigation condition.

The driver stopping at an intersection for an inappropriately long period of time whilecollecting navigation information was the second type of unsafe stop that was analyzed. While the danger was considerably lower than some of the other safety errors considered, this behavior did have some accident potential associated with it. For example, depending on the speed of the associated roadway, being stopped at a green light could result in a serious rear-end collision. Figure 58 shows the number of this type of unsafe stop incident. This type of error occurred 37 times, which was more than double that of the more serious unsafe stopping in traffic type with 14 errors. 


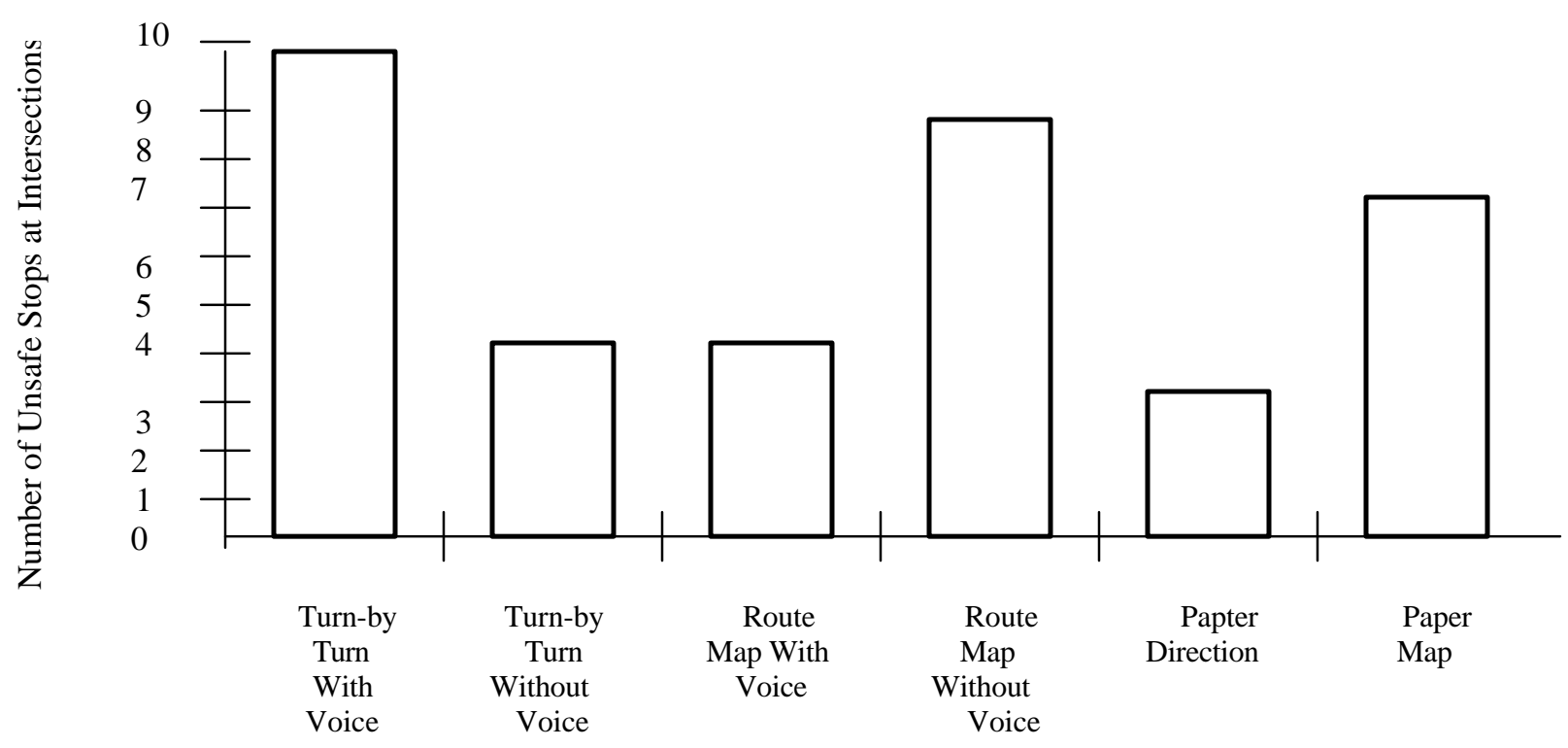

Figure 58. Total number of unsafe or extended stops at intersections where the user needed to gather navigation information. Shown for each navigation condition.

There was a higher group of incident rates for the three display conditions, turn-by-turn with voice, route map without voice, and paper map. The last two conditions fall into the established pattern of a higher workload associated with those navigation aids, but the high incidents in turn-by-turn with voice is difficult to explain. Note, that the number of incidents was low and should be addressed with caution. This event did, however, occur across a range of subjects, and no one subject repeated the error with great frequency.

\section{Dangerously Close Headways}

Incidents of unsafe headway between the camera car and a leading vehicle were viewed as indicators of the subjects' comfort with a display condition or as a cue to increased workload. Incidents of slow speed that were previously discussed, indicated that as workload increased speed tended to decrease. Similarly, drivers are very likely to increase headway if operating under visual or cognitive attention extremes which are focused away from the forward roadway. In addition, subjects might have realized that they were not directing enough attention forward and that following too closely represented a greater danger in these conditions. On the other hand, if the visual attention required using a navigation condition was low, the operator tended to drive at their normal following distance. Quite often these "normal" following distances fell within the unsafe headway classification due to poor training and habits. This exact trend is shown in figure 59. The paper map is the most familiar method of navigation and corresponds to the display with the eight incidents of unsafe headway. The two turn-by-turn conditions appear equal in the figure, with six incidents for voice and five for no voice. The route map conditions, which required more visual attention, had only one incident each for voice and no voice. It is apparent from previous findings discussed in this report that subjects used good judgment in determining their overload, and adjusted their headway spacing accordingly. Note, however, that increases in headway are not necessarily safer if it comes at the cost of increasing workload to a level that could create a hazard in other driving situations. 


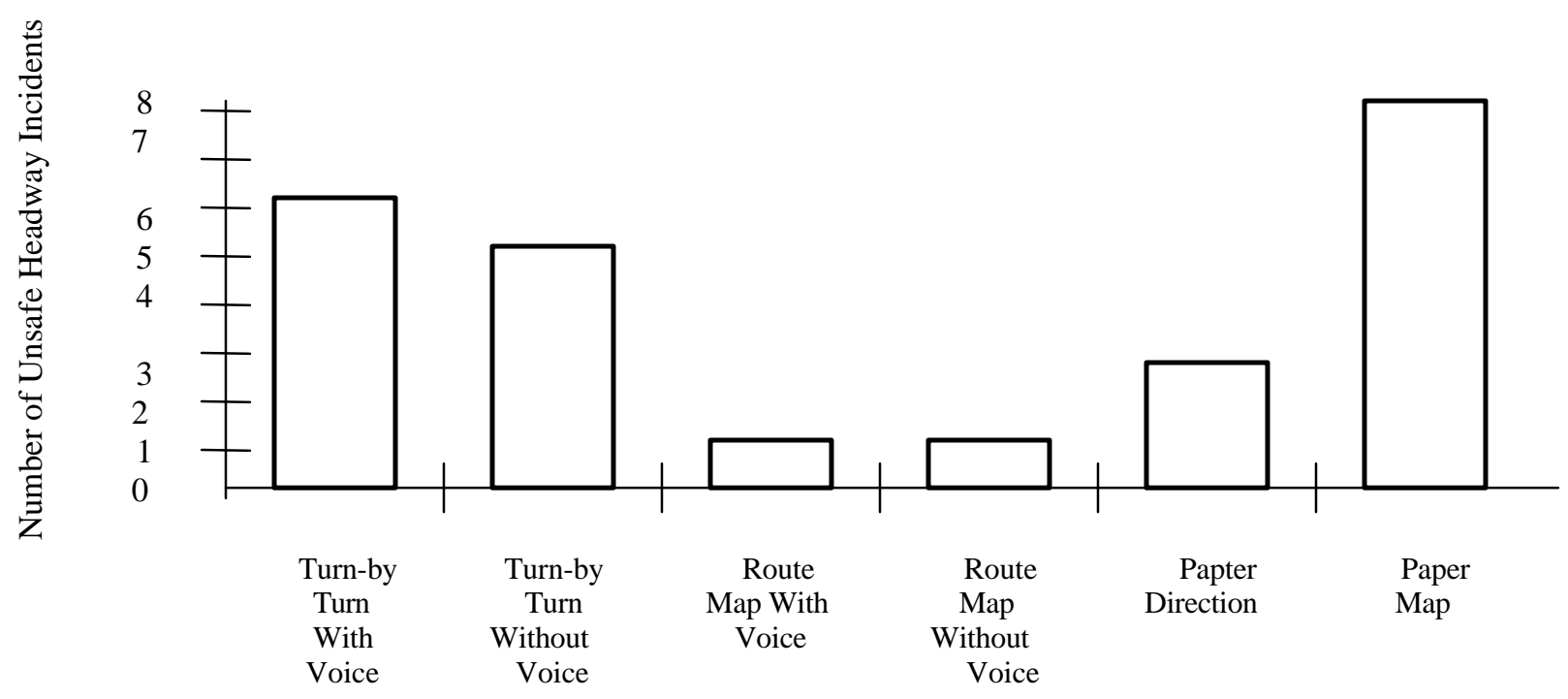

Figure 59. Total number of unsafe headway incidents committed within each navigation condition.

\section{Subjective Workload Ratings for Overload}

Subjective workload ratings where all three ratings were in the high category related to safety in that the driver felt overloaded, which could in turn have led to some performance decrement in the driving task. An analysis of these high ratings across the different navigational conditions is shown in figure 60 . The trends established by many other measures of performance are considerably different than workload ratings for the varying display configurations. The paper map control condition had the greatest number of high triple category workload ratings incidents, with 32 total occurrences. This finding contra dicts other safety findings. Turn-by-turn with voice, a display that in other measures was comparable to the paper-map condition, was eight times lower in this measure, with only four occurrences. Previously analyzed safety measures have shown that turn-by-turn with voice leads to a safe level of driving behavior in comparison to other conditions (other than headway), and these results support that trend. Turn-by-turn without voice and paper directions also had a low number of incidents, with three and one respectively. The route map condition also followed the trend of being higher in attention demand with 15 ratings indicating overload.

Subjective ratings measure a somewhat different but related construct than safety. Work-load or cognitive attention can be high, and thus stressful to the operator and yet cause no driving performance decrement or safety-related error. This is the likely explanation of the results seen here. The user of the paper map is more stressed due to time pressure or navigation error occurrence and therefore rates workload high. However, the paper map has been shown to require little visual attention demand, and it is apparent that this measure losely to safety per se for driving than workload. The paper map navigation method is not as convenient or usable as other methods, but its lack of usability did not result in a measurable safety decrement. 


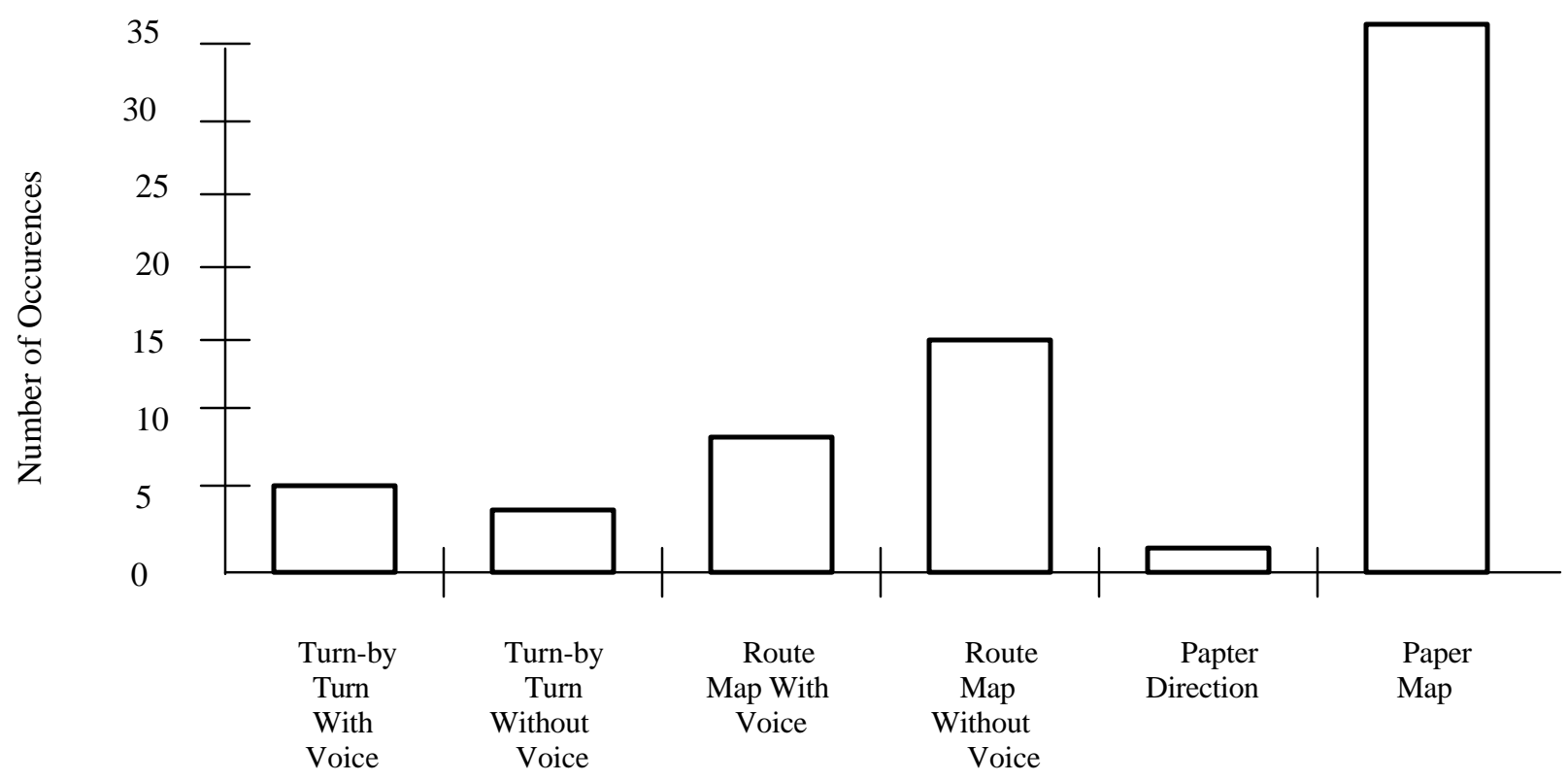

Figure 60. Total number of occurrences where all three classifications of subjective workload were rated as high. Shown for each navigation condition.

\section{General Risk Assessment Analysis}

The Failure Modes Effects and Criticality Analysis (FMECA) utilized in the industrial safety field was used as a model for combining these research findings. Application of this methodology to driving safety was an experimental undertaking. An objective method has been formulated to combine the measures of environmental proximity, potential severity, and number of incidents in order to assess the safety implications across all the navigational display conditions. This has provided a hazard risk measure that can be used to globally distinguish navigation conditions from one another. It will also allow the results to be quantified against true accident rates, if a source of epidemiological data can be found that is specific enough to determine accident rates for drivers navigating to unfamiliar locations using either a paper map or paper directions. As mentioned previously, the task of navigating is considerably more difficult than normal driving, so normal accident data would not be a valid comparison measure.(3) This specific source of epidemiological data has not been located, and probably does not exist.

Figure 61 shows a matrix that was used to assess the final hazard risk for each event based on its associated combination of an environmental proximity and potential severity. By combining all the events, a more objective assessment can be made between the display configurations. However, the differentiation assignment between acceptable or unacceptable risk is a subjective criterion and should be based upon real-world accident frequencies. It should also be noted that this procedure could be accomplished with only the events that were caused by attention focused on the navigation display. However, this was not done for two reasons; first, total count of some events are biased since they are only collected when a display glance occurs (glances $>2.5 \mathrm{~s}$ ), and second, because valuable information may be lost if non-navigation attention based errors are deleted. By utilizing the total number of all events for each navigational condition, a conclusion can be drawn about the total index of criticality for the safety-related events within each navigational condition. 


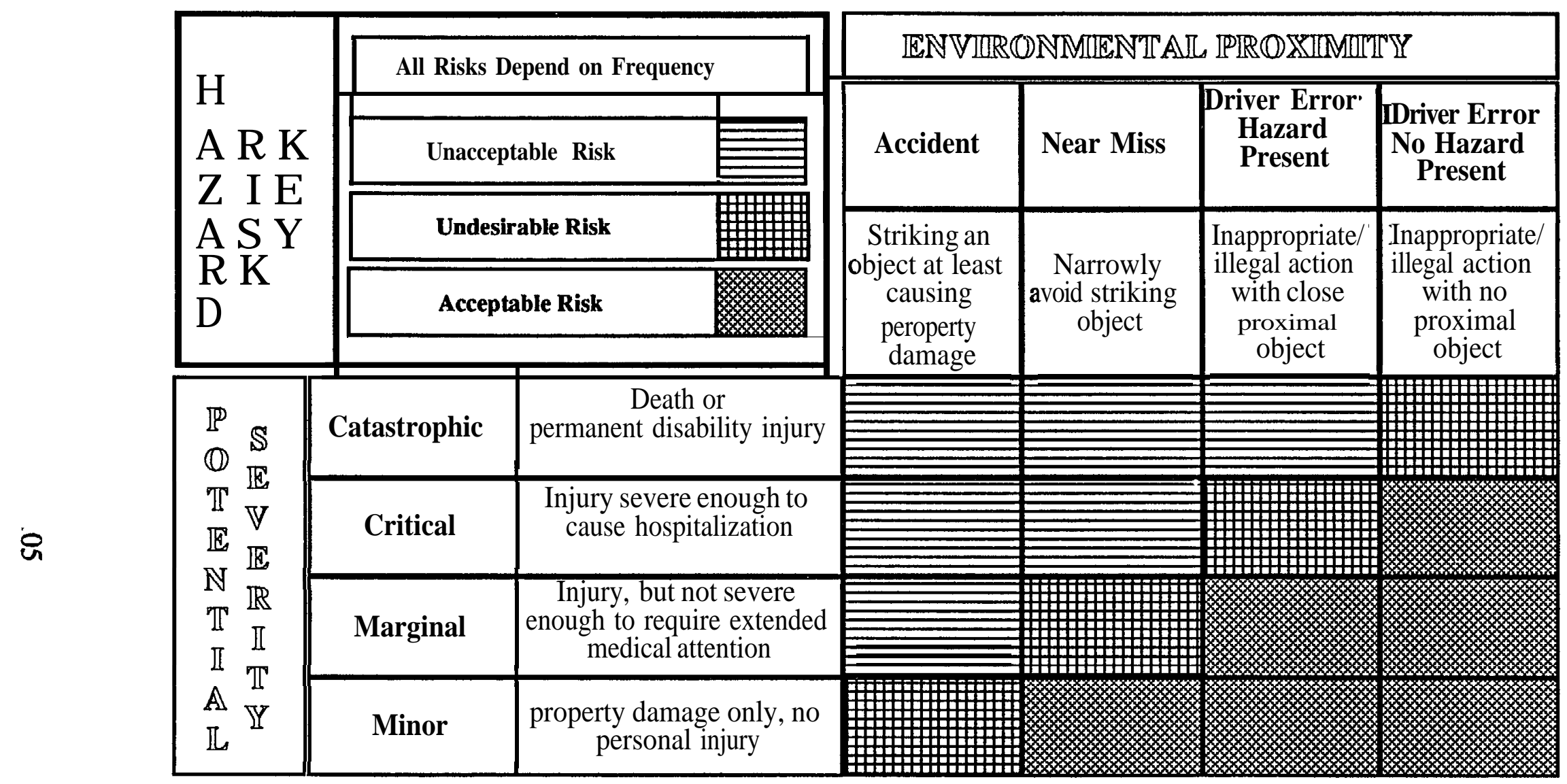

Figure 61. Risk assessment matrix for the safety analysis; determines the risk associated with all combinations of the probable severity of an accident and the environmental proximity at which each safety related event occured. 
The results of the final hazard risk assessment (see figure 62) follow the trends discussed within each of the individual performance measures. Overall, the turn-by-turn with voice condition appeared to have the same associated risks as the paper map and paper directions. Figure 62 shows the distributions of unacceptable risk, undesirable risk, and acceptable risk. Route map without voice had the most unacceptable risk events with a total of 26 . It also had the highest number of undesirable risks with 74 and acceptable risks with 132 . The paper map condition had only 2 unacceptable risk values, 30 undesirable risk incidents, and 77 acceptable with review risks. This compares directly with the paper directions, where 6 unacceptable risk events occurred, 39 undesirable, and 85 acceptable risk events. The TravTek display condition of turn-by-turn with voice had essentially the same associated risks; 8 unacceptable, 39 undesirable, and 79 acceptable. A second grouping of risk assessment values occurred for the remaining two TravTek displays. Turn-by-turn without voice had 17 undesirable risk values, while route map with voice had a comparable 15. For undesirable risk values, turn-by-turn without voice had 55 events route map, while with voice had 35 . The turn-by-turn without voice and route map with voice for risk values were 92 and 87 respectively.

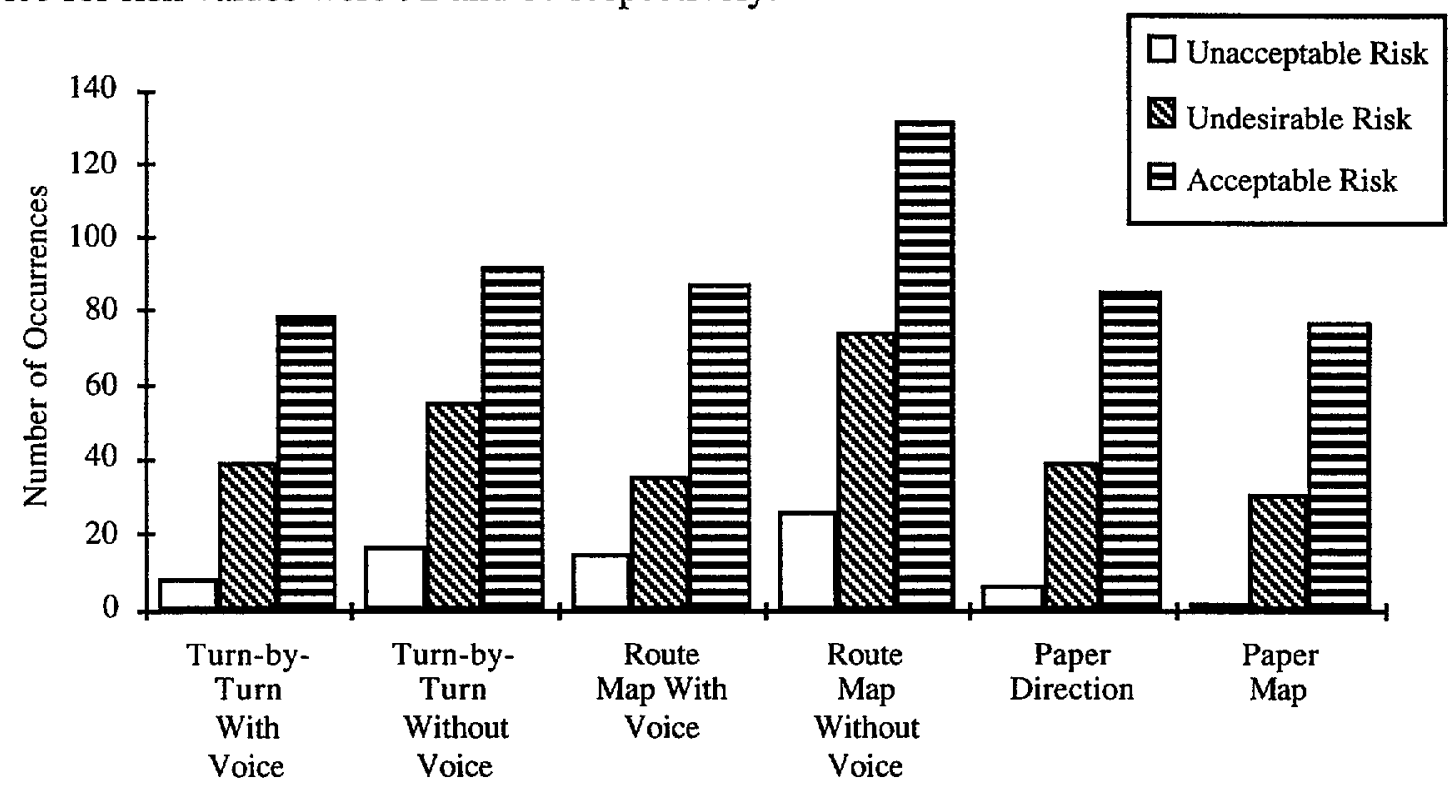

Figure 62. Total count of all final hazard risk assessment values computed for each safetyrelated error. Shown by level of risk and each navigation condition.

\section{Discussion}

Several conclusions were drawn after considering the set of different performance measures taken to evaluate the safety of each navigational condition. The largest number of unsafe incidents occurred during the use of the route map without voice condition. Based upon the risk assessment analysis and the individual analyses, the route map without voice condition was the least safe configuration. The differences in risk between the route map without voice display and the current standard paper map or paper direction seemed too great to justify recommending implementation without supplementary voice information or a turn-by-turn guidance option. It should be noted that this study forced the sole use of single display configurations for navigating to a destination. The actual TravTek system uses turn-by-turn with voice as a default, and the route map modes are driver requested to provide supplementary information. 
The route map without voice condition had the greatest number of unsafe incidents in glances over $2.5 \mathrm{~s}$, lane deviations, and inappropriate speed performance measures. All of these results support the conclusion that this display requires a high degree of visual attention in order to utilize the presented information. Adding the element of voice guidance to the route map display generally improved performance to the level of several other navigation conditions. This leads to the conclusion that providing auditory turn-by-turn information reduces the degree to which visual interface is overloading the operator. Subjective workload ratings also support this conclusion.

As additional evidence in the driving task intrusion section confirms, the route map without voice condition had the highest visual attention demand overall. Note that some measures (in particular the subjective workload assessments) showed the paper map condition as having the highest overall workload despite the relatively low visual attention demand requirements. These results indicate that visual attention demand has the greatest impact on driving safety, while cognitive attention or mental workload has less impact. This finding is somewhat intuitive given that driving is primarily a visual task.

Adding voice navigation information to a TravTek visual display also improved performance for the turn-by-turn condition. In the majority of error types and in the overall error analysis, turn-by-turn with voice had a lower number of safety errors and incidents than turn-by-turn without voice. There are a few safety-related incidents that do not support this trend. Turn-by-turn with voice had a higher number of inappropriate stopped behaviors at intersections and a greater number of unsafe lateral maneuver incidents. In each of these cases, the number of incidents was small and somewhat unreliable.

Since the paper map and paper direction list are currently available to drivers, they were used as control conditions against which to compare the relative safety of the TravTek conditions. In general, both the paper map and paper direction list conditions had a small number of incidents compared to the TravTek conditions. With respect to safety and compared to the current methods of navigating, the turn-by-turn with voice condition faired favorably. In addition, the turn-by-turn without voice and route map with voice conditions, although having somewhat higher numbers of incidents than the control conditions, were comparable for many of the safety-related measures.

Although the paper map condition compared favorably in a number of safety incidents, there were still some safety concerns. Incidents of high workload ratings were more frequent than with the other navigation conditions. It is clear from the driving task intrusion and navigation performance sections that the paper map condition was by far the least usable of the navigation conditions. This resulted in poorer navigation performance and a higher workload in comparison to the other conditions. It is interesting to note from these results, that the drivers apparently adapted to this awkward navigation means without driving unsafely. This is supported by the results that paper map drivers drove slower and pulled over to stop more often than in other conditions. In addition, the paper map condition subjects pulled off in safe circumstances in all but two occasions.

Finally, a reminder that all drivers adapted relatively well to the forced use of single navigation display formats. The actual TravTek system gave drivers a choice of configurations with the press of a button, and results of the Rental User and Local User studies showed that drivers most often selected the turn-by-turn with voice configuration.(28) Clearly, an ability to select a configuration will mitigate some of the safety concerns expressed for the route map without voice configuration. Even with these concerns, there were few serious near misses and no accidents. When external events occurred, drivers tended to react appropriately, and avoid hazards. While there are true 
performance differences with regard to the safe use of the different navigation configurations, the overall results show a great deal of adaptability by users.

\section{ISSUE 5: DO DRIVING PERFORMANCE, NAVIGATION PERFORMANCE, AND DRIVING SAFETY VARY AS A FUNCTION OF TRAVTEK EXPERIENCE?}

This section includes the measures that are used to assess differences between novice and experienced drivers. "Novice" drivers are defined as subjects with no previous TravTek system experience while performing an initial Camera Car Study. Again, the purpose of the Camera Car Study was to provide a detailed evaluation of driver performance and behavior while operating the TravTek system. "Experienced" drivers are defined as subjects that have used the system for their first camera car experimental drive and have used the system everyday for 6 weeks during the Local User Study. Note, however, that differences in driver experience were present since they had used the system for varying amounts of time during the 6 week test period. These differences are discussed in the navigation condition usage between experience levels section that follows.

\section{Navigation Condition Usage Between Experience Levels}

Between the time when local users performed their first and second test drives, data were collected for the number of times that the NAVIG button was used to activate the TravTek system, and the amount of time that was spent using each navigation condition. These data provide an idea of the amount of experience that the local users gained between their first and second experimental drives.

Overall, the combined time in use varied between subjects, with the minimum being 41 min and the maximum being $28 \mathrm{~h}$ (see table 11). The number of times the NAVIG button was operated also varied from 267 to 17 times.

Table 11. Time local users used the different navigation conditions.

\begin{tabular}{|c|c|c|c|c|c|c|c|c|c|c|c|c|}
\hline \multirow{2}{*}{$\begin{array}{l}\text { Subject } \\
\text { Id \# }\end{array}$} & \multirow[b]{2}{*}{ Gender } & \multirow{2}{*}{$\begin{array}{c}\text { Age } \\
\text { Groupd }\end{array}$} & \multirow[b]{2}{*}{ ount } & \multicolumn{2}{|c|}{$\begin{array}{l}\text { Time } \\
\text { TNoice }\end{array}$} & \multicolumn{2}{|l|}{$\begin{array}{r}\text { Time } \\
\text { ST/Voice }\end{array}$} & \multicolumn{2}{|l|}{ Time } & \multicolumn{2}{|c|}{ Time } & \multirow{2}{*}{$\begin{array}{l}\text { Combined } \\
\text { Time in Use } \\
\text { Total }\end{array}$} \\
\hline & & & & Mean & $\%$ & Mean & $\%$ & Mean & $\%$ & Mean & $\%$ & \\
\hline 11411 & Female & 35 to 45 & 230 & $6: 28$ & 47 & 5:30 & 38 & $1: 33$ & 11 & $: 38$ & 4 & $12: 35: 42$ \\
\hline 11451 & ale & 35 to 45 & 267 & $1: 00$ & 8 & $1: 04$ & 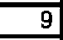 & $2: 51$ & 23 & $7: 05$ & 61 & $2: 55: 21$ \\
\hline 11481 & Male & 35 to 45 & 42 & $1: 38$ & 41 & $3: 10$ & 44 & $: 09$ & & $1: 05$ & 12 & $1: 32: 47$ \\
\hline 11511 & Female & 35 to 45 & 135 & $13: 44$ & 93 & $: 22$ & 1 & 51 & 6 & $: 14$ & 1 & $28: 51: 30$ \\
\hline 11541 & Male & 35 to 45 & 103 & $1: 17$ & 15 & $1: 36$ & 18 & $1: 13$ & 14 & $4: 45$ & 54 & $5: 31: 14$ \\
\hline 11601 & Male & $65+$ & 67 & $1: 47$ & 26 & $1: 33$ & 22 & $1: 47$ & 26 & $1: 46$ & 26 & $1: 55: 42$ \\
\hline & Female & $65+$ & 22 & $3: 15$ & 71 & $: 40$ & 15 & $: 30$ & 11 & $: 09$ & 3 & $2: 30: 48$ \\
\hline & ale & $65+$ & 17 & $9: 26$ & 77 & $1: 11$ & 10 & $1: 37$ & 13 & $: 00$ & $\overline{0}$ & 2:09:0 \\
\hline 11 & ie & $65+$ & 75 & $9: 22$ & 93 & $: 04$ & 1 & $: 40$ & $\overline{7}$ & $: 03$ & $\overline{0}$ & $10: 57: 0$ \\
\hline 11721 & Female & $65+$ & 19 & $1: 23$ & 19 & $1: 13$ & 17 & $1: 23$ & 19 & $3: 10$ & 45 & $41: 02$ \\
\hline 11751 & Female & $65+$ & 45 & $16: 51$ & 85 & $1: 28$ & 6 & $1: 43$ & 0 & $: 06$ & 1 & $10: 51: 31$ \\
\hline 16301 & Female & 35 to 45 & 164 & $1: 09$ & 13 & $6: 57$ & 82 & $: 10$ & 1 & $: 21$ & 4 & $16: 01: 4$ \\
\hline Average & & & 1186 & $1: 07: 20$ & 49 & $6: 48$ & & $14: 27$ & 12 & 19:2 & 18 & \\
\hline
\end{tabular}

Subjects were asked to use each of the turn-by-turn with voice, turn-by-turn without voice, route map with voice, and route map without voice navigation conditions at least once each day. Table 11 shows that the combined mean percentage of time that each navigation condition was used was not evenly distributed. The turn-by-turn with voice condition was used approximately half of the time that subjects spent gaining experience. Because the proportion of time that each navigation condition was used was not evenly distributed, it is assumed local users chose the display used based on preference, system defaults, or some 
other criteria. The majority of the time, most users chose to use one primary navigation condition, while only occasionally choosing to use other conditions.

\section{Measures of Performance for Driving Performance}

Two separate ANOVA's were conducted to assess the effects of system experience. Due to the repeated measure design of the experiment and the presence of some missing data (12 out of 252 data runs could not be analyzed for various reasons), the variables of interest could not be combined into a single ANOVA, since some of the error terms ran short of degrees of freedom. Consequently, two sets of two-way ANOVA's were conducted. The first set addressed experience by age, and the second set analyzed experience by navigation condition. Because both of the two-way interactions performed in these analyses are of interest to this study, this approach was taken despite the inherent type I error inflation. Therefore, readers are cautioned in how they interpret significant single results near the criterion value of $\mathrm{p}=0.05$.

The results of the ANOVA's performed to analyze driving performance are listed in tables 12 and 13. Table 12 addresses driver performance measures as a function of age and experience. Table 13 addresses driver performance as a function of experience and navigation condition. The measures found statistically significant between novice and experienced users include duration of glances to the navigation system and number of steering reversals corrected by travel time. Also found statistically significant were the interactions between experience and navigation condition for mean speed, number of brake applications corrected for travel time, and number of steering reversals corrected for travel time. Other driving performance variables that were analyzed, such as speed variance, steering wheel position variance, lateral and longitudinal acceleration, time spent scanning the roadway environment, and number of brake applications, did not show statistically significant differences as experience increased.

\section{Driver Eye Glance Behavior}

Figure 63 shows the mean duration of glances to the navigational aids. The experienced drivers glanced at the navigation system fewer times (4757 compared to 5578 glances) and for significantly shorter durations than novice drivers. Figure 64 shows the total combined number of glances for novice and experienced drivers.

Figure 65 shows the number of roadway-related glances that drivers performed by level of experience. Experienced drivers had a greater number of glances to the roadway environment than novice drivers.

\section{Mean Speed}

Figure 66 shows the significant interaction between experience and navigation condition for mean speed. Recall from previous sections that lower mean speed is indicative of greater attention demand. The turn-by-turn without voice, route map without voice, and paper map conditions showed increases in the mean speed with increased experience. The turnby-turn with voice, route map with voice and paper direction navigation conditions showed that with experience, there were decreases in the mean speed. The paper map condition had the largest difference between trials, with subjects driving faster on their second experimental drive. 
Table 12. ANOVA's for driver performance measures addressing driver age.

Duration of Glances to Navigation Aid

\begin{tabular}{|l|r|r|r|r|}
\hline Source & \multicolumn{1}{|c|}{ DF } & \multicolumn{1}{|c|}{ MS } & \multicolumn{1}{|c|}{ F } & P \\
\hline Local users' first drive \& Local users' second drive & 1 & 24.7107 & 13.80 & 0.0040 \\
\hline Age group & 1 & 0.0907 & 0.00 & 0.9487 \\
\hline $\begin{array}{l}\text { Local users' first drive \& Local users' second drive X Age } \\
\text { group }\end{array}$ & 1 & 0.5249 & 0.29 & 0.6011 \\
\hline Subjects (Age Group) & 10 & 20.8419 & & \\
\hline $\begin{array}{l}\text { Local users' first drive \& Local users' second drive X } \\
\text { Subjects (Age group) }\end{array}$ & 10 & 1.7908 & & \\
\hline
\end{tabular}

Duration of Roadway Related Glances

\begin{tabular}{|l|r|r|r|r|}
\hline Source & DF & \multicolumn{1}{|c|}{ MS } & F & P \\
\hline Local users' first drive \& Local users' second drive & 1 & 72.6285 & 1.12 & 0.3149 \\
\hline Age group & 1 & 1885.2536 & 9.37 & 0.0120 \\
\hline $\begin{array}{l}\text { Local users' first drive \& Local users' second drive X Age } \\
\text { group }\end{array}$ & 1 & 46.0461 & 0.71 & 0.4192 \\
\hline Subjects (Age Group) & 10 & 201.1478 & & \\
\hline $\begin{array}{l}\text { Local users' first drive \& Local users' second drive X } \\
\text { Subjects (Age group) }\end{array}$ & 10 & 64.8595 & & \\
\hline
\end{tabular}

\section{Variance in Lateral Acceleration}

\begin{tabular}{|c|c|c|c|c|}
\hline Source & DF & MS & $\mathrm{F}$ & $\mathrm{P}$ \\
\hline Local users' first drive \& Local users' second drive & 1 & 0.0000003 & 0.24 & 0.6363 \\
\hline Age group & 1 & 0.00015 & 2.16 & 0.1724 \\
\hline $\begin{array}{l}\text { Local users' first drive \& Local users' second drive X Age } \\
\text { group }\end{array}$ & 1 & 0.000000 & 0.00 & 0.9675 \\
\hline Subjects (Age Group) & 10 & 0.000007 & & \\
\hline $\begin{array}{l}\text { Local users' first drive \& Local users' second drive X } \\
\text { Subjects (Age group) }\end{array}$ & 10 & 0.000014 & & \\
\hline \multicolumn{5}{|l|}{ Variance in Steering Position } \\
\hline Source & $\mathrm{DF}$ & MS & $\mathrm{F}$ & $P$ \\
\hline Local users' first drive \& Local users' second drive & 1 & 167785.40 & 0.42 & 0.5299 \\
\hline Age group & 1 & 95146.11 & 0.23 & 0.6386 \\
\hline $\begin{array}{l}\text { Local users' first drive \& Local users' second drive X Age } \\
\text { group }\end{array}$ & 1 & 760840.83 & 1.92 & 0.1959 \\
\hline Subjects (Age Group) & 10 & 405760.80 & & \\
\hline $\begin{array}{l}\text { Local users' first drive \& Local users' second drive X } \\
\text { Subjects (Age group) }\end{array}$ & 10 & 396167.14 & & \\
\hline
\end{tabular}


Table 12. ANOVA's for driver performance measures addressing driver age (continued)

Variance in Longitudinal Acceleration

\begin{tabular}{|c|c|c|c|c|}
\hline Source & $\mathrm{DF}$ & MS & $\mathrm{F}$ & $\mathrm{P}$ \\
\hline Local users' first drive \& Local users' second drive & 1 & 0.0000073 & 3.85 & 0.0783 \\
\hline Age group & 1 & 0.0000078 & 4.09 & 0.0706 \\
\hline $\begin{array}{l}\text { Local users' first drive \& Local users' second drive X Age } \\
\text { group }\end{array}$ & 1 & 0.0000002 & 1.04 & 0.3323 \\
\hline Subjects (Age Group) & 10 & 0.0000190 & & \\
\hline $\begin{array}{l}\text { Local users' first drive \& Local users' second drive X } \\
\text { Subjects (Age group) }\end{array}$ & 10 & 0.0000019 & & \\
\hline
\end{tabular}

Mean Speed

\begin{tabular}{|l|r|r|r|r|}
\hline Source & DF & \multicolumn{1}{|c|}{ MS } & \multicolumn{1}{|c|}{ F } & P \\
\hline Local users' first drive \& Local users' second drive & 1 & 9.4355 & 1.28 & 0.2843 \\
\hline Age group & 1 & 275.5329 & 9.59 & 0.0113 \\
\hline $\begin{array}{l}\text { Local users' first drive \& Local users' second drive X Age } \\
\text { group }\end{array}$ & 1 & 0.3577 & 0.05 & 0.8301 \\
\hline Subjects (Age Group) & 10 & 28.7356 & & \\
\hline $\begin{array}{l}\text { Local users' first drive \& Local users' second drive X } \\
\text { Subjects (Age group) }\end{array}$ & 10 & 7.3715 & & \\
\hline
\end{tabular}

\section{Speed Variance}

\begin{tabular}{|c|c|c|c|c|}
\hline Source & DF & MS & $\mathrm{F}$ & $\mathrm{P}$ \\
\hline Local users' first drive \& Local users' second drive & 1 & 1 & 3177.6678 & 0.3853 \\
\hline Age group & 1 & 66589.4960 & 9.63 & 0.0112 \\
\hline $\begin{array}{l}\text { Local users' first drive \& Local users' second drive X Age } \\
\text { group }\end{array}$ & 1 & 62.8604 & 0.02 & 0.9009 \\
\hline Subjects (Age Group) & 10 & 69168.2053 & & \\
\hline $\begin{array}{l}\text { Local users' first drive \& Local users' second drive X } \\
\text { Subjects (Age group) }\end{array}$ & 10 & 38552.0209 & & \\
\hline \multicolumn{5}{|l|}{ Negative Longitudinal Acceration } \\
\hline Source & $\mathrm{DF}$ & MS & $\mathrm{F}$ & $P$ \\
\hline Local users' first drive \& Local users' second drive & 1 & 0.000043 & 0.80 & 0.3915 \\
\hline Age group & 1 & 0.000695 & 0.76 & 0.4031 \\
\hline $\begin{array}{l}\text { Local users' first drive \& Local users' second drive X Age } \\
\text { group }\end{array}$ & 1 & 0.000002 & 0.03 & 0.8662 \\
\hline Subjects (Age Group) & 10 & 0.000912 & & \\
\hline $\begin{array}{l}\text { Local users' first drive \& Local users' second drive X } \\
\text { Subjects (Age group) }\end{array}$ & 10 & 0.000054 & & \\
\hline
\end{tabular}


Table 12. ANOVA's for driver performance measures addressing driver age (continued)

Variance of Negative Longitudinal Accleration

\begin{tabular}{|l|r|r|r|r|}
\hline Source & DF & \multicolumn{1}{|c|}{ MS } & \multicolumn{1}{|c|}{ F } & P \\
\hline Local users' first drive \& Local users' second drive & 1 & 0.00000016 & 0.08 & 0.7835 \\
\hline Age group & 1 & 0.00001802 & 1.42 & 0.2603 \\
\hline $\begin{array}{l}\text { Local users' first drive \& Local users' second drive X Age } \\
\text { group }\end{array}$ & 1 & 0.00000006 & 0.31 & 0.5886 \\
\hline Subjects (Age Group) & 10 & 0.00012655 & & \\
\hline $\begin{array}{l}\text { Local users' first drive \& Local users' second drive X } \\
\text { Subjects (Age group) }\end{array}$ & 10 & 0.00000199 & & \\
\hline
\end{tabular}

Number of Brake Applications Corrected by Travel Time

Source

Local users' first drive \& Local users' second drive

Age group

Local users' first drive \& Local users' second drive X Age

group

Subjects (Age Group)

Local users' first drive \& Local users' second drive $\mathrm{X}$

Subjects (Age group)

Number of Steering Reversals Corrected by Travel Time

Source

Local users' first drive \& Local users' second drive

Age group

Local users' first drive \& Local users' second drive X Age

group

Subjects (Age Group)

Local users' first drive \& Local users' second drive $\mathrm{X}$

Subjects (Age group)

\begin{tabular}{r|r|r|r|}
\hline \multicolumn{1}{|l|}{ DF } & \multicolumn{1}{|c|}{ MS } & F & P \\
\hline 1 & 0.0022 & 1.11 & 0.3186 \\
\hline 1 & 0.0045 & 0.31 & 0.5884 \\
\hline 1 & 0.0001 & 0.57 & 0.4684 \\
\hline 10 & 0.0014 & \multicolumn{3}{|l|}{} \\
\hline
\end{tabular}

Time for Which the Brake is Presssed

Source

Local users' first drive \& Local users' second drive

Age group

Local users' first drive \& Local users' second drive X Age

group

Subjects (Age Group)

Local users' first drive \& Local users' second drive $\mathrm{X}$

Subjects (Age group)

\begin{tabular}{|r|r|r|r|}
\hline DF & MS & F & P \\
\hline 1 & 0.0090 & 8.66 & 0.0164 \\
\hline 1 & 0.0001 & 0.00 & 0.9659 \\
\hline 10 & 0.0040 & 3.86 & 0.0810 \\
\hline 10 & 0.0090 & & \\
& & \multicolumn{3}{|c|}{} \\
\hline
\end{tabular}

\begin{tabular}{|l|r|r|r|r|}
\hline & DF & \multicolumn{1}{|c|}{ MS } & F & P \\
\hline & 1 & 7.0882 & 0.00 & 0.9643 \\
\hline & 1 & 3020.9575 & 0.44 & 0.5230 \\
\hline & 1 & 650.7166 & 0.19 & 0.6694 \\
\hline & 10 & 6894.8189 & & \\
\hline
\end{tabular}


Table 13. ANOVA's for driver performance measures addressing navigation condition.

Duration of Glances to Navigation Aid

\begin{tabular}{|l|r|r|r|r|}
\hline Source & \multicolumn{1}{|c|}{$\mathrm{dF}$} & \multicolumn{1}{|c|}{ MS } & \multicolumn{1}{|c|}{ P } \\
\hline Local users' first drive \& Local users' second drive & 1 & 13.6550 & 28.08 & 0.0003 \\
\hline Navigation condition & 5 & 7.3694 & 7.75 & 0.0001 \\
\hline $\begin{array}{l}\text { Local users' first drive \& Local users' second drive X } \\
\text { Navigation condition }\end{array}$ & 5 & 1.3015 & 1.85 & 0.1210 \\
\hline $\begin{array}{l}\text { Local users' first drive \& Local users' second drive X } \\
\text { Subjects }\end{array}$ & 11 & 0.4863 & & \\
\hline Navigation condition X Subjects & 55 & 0.9503 & \\
\hline $\begin{array}{l}\text { Local users' first drive \& Local users' second drive X } \\
\text { Navigation condition X Subjects }\end{array}$ & 47 & 0.7028 & & \\
\hline
\end{tabular}

Duration of Roadway Related Glance

\begin{tabular}{|c|c|c|c|c|}
\hline Source & $\mathrm{dF}$ & MS & $\mathrm{F}$ & $\mathrm{P}$ \\
\hline Local users' first drive \& Local users' second drive & 1 & 58.9545 & 1.27 & 0.2845 \\
\hline Navigation condition & 5 & 226.9886 & 5.59 & 0.0003 \\
\hline $\begin{array}{l}\text { Local users' first drive \& Local users' second drive } \mathrm{X} \\
\text { Navigation condition }\end{array}$ & 5 & 20.5056 & 1.11 & 0.3660 \\
\hline $\begin{array}{l}\text { Local users' first drive \& Local users' second drive X } \\
\text { Subjects }\end{array}$ & 11 & 46.5784 & & \\
\hline Navigation condition X Subjects & 55 & 40.6245 & & \\
\hline $\begin{array}{l}\text { Local users' first drive \& Local users' second drive X } \\
\text { Navigation condition X Subjects }\end{array}$ & 47 & 865.2709 & & \\
\hline \multicolumn{5}{|l|}{ Variance in Lateral Acceleration } \\
\hline Source & $\mathrm{dF}$ & MS & $\mathrm{F}$ & $\mathrm{P}$ \\
\hline Local users' first drive \& Local users' second drive & 1 & 0.0000003 & 0.24 & 0.6364 \\
\hline Navigation condition & 5 & 0.0000016 & 1.59 & 0.1792 \\
\hline $\begin{array}{l}\text { Local users' first drive \& Local users' second drive } \mathrm{X} \\
\text { Navigation condition }\end{array}$ & 5 & 0.0000010 & 0.90 & 0.1792 \\
\hline $\begin{array}{l}\text { Local users' first drive \& Local users' second drive X } \\
\text { Subjects }\end{array}$ & 11 & 0.0000012 & & \\
\hline Navigation condition X Subjects & 55 & 0.0000010 & & \\
\hline $\begin{array}{l}\text { Local users' first drive \& Local users' second drive X } \\
\text { Navigation condition X Subjects }\end{array}$ & 49 & 0.0000011 & & \\
\hline
\end{tabular}


Table 13. ANOVA's for driver performance measures addressing navigation condition (continued).

\begin{tabular}{|c|c|c|c|c|}
\hline \multicolumn{5}{|l|}{ Variance in Steering Position } \\
\hline Source & $\mathrm{dF}$ & MS & $\mathrm{F}$ & $\mathrm{P}$ \\
\hline Local users' first drive \& Local users' second drive & 1 & 147609.79 & 0.35 & 0.5652 \\
\hline Navigation condition & 5 & 171433.96 & 2.05 & 0.0856 \\
\hline $\begin{array}{l}\text { Local users' first drive \& Local users' second drive X } \\
\text { Navigation condition }\end{array}$ & 5 & 126778.44 & 1.18 & 0.3325 \\
\hline $\begin{array}{l}\text { Local users' first drive \& Local users' second drive } \mathrm{X} \\
\text { Subjects }\end{array}$ & 11 & 419733.42 & & \\
\hline Navigation condition X Subjects & 55 & 83556.76 & & \\
\hline $\begin{array}{l}\text { Local users' first drive \& Local users' second drive X } \\
\text { Navigation condition X Subjects }\end{array}$ & 49 & 107433.90 & & \\
\hline \multicolumn{5}{|l|}{ Variance in Longitudinal Acceleration } \\
\hline Source & $\mathrm{dF}$ & MS & $\mathrm{F}$ & $\mathrm{P}$ \\
\hline Local users' first drive \& Local users' second drive & 1 & 0.0000064 & 3.21 & 0.1005 \\
\hline Navigation condition & 5 & 0.0000018 & 0.87 & 0.5045 \\
\hline $\begin{array}{l}\text { Local users' first drive \& Local users' second drive } \mathrm{X} \\
\text { Navigation condition }\end{array}$ & 5 & 0.0000014 & 1.10 & 0.3751 \\
\hline $\begin{array}{l}\text { Local users' first drive \& Local users' second drive X } \\
\text { Subjects }\end{array}$ & 11 & 0.0000019 & & \\
\hline Navigation condition X Subjects & 55 & 0.0000021 & & \\
\hline $\begin{array}{l}\text { Local users' first drive \& Local users' second drive X } \\
\text { Navigation condition X Subjects }\end{array}$ & 49 & 0.0000012 & & \\
\hline \multicolumn{5}{|l|}{ Mean Speed } \\
\hline Source & $\mathrm{dF}$ & MS & $\mathrm{F}$ & $\mathrm{P}$ \\
\hline Local users' first drive \& Local users' second drive & 1 & 9.0273 & 1.16 & 0.3040 \\
\hline Navigation condition & 5 & 10.0046 & 1.22 & 0.3106 \\
\hline $\begin{array}{l}\text { Local users' first drive \& Local users' second drive } \mathrm{X} \\
\text { Navigation condition }\end{array}$ & 5 & 14.9691 & 2.72 & 0.0304 \\
\hline $\begin{array}{l}\text { Local users' first drive \& Local users' second drive X } \\
\text { Subjects }\end{array}$ & 11 & 7.7661 & & \\
\hline Navigation condition $\mathrm{X}$ Subjects & 55 & 8.1775 & & \\
\hline $\begin{array}{l}\text { Local users' first drive \& Local users' second drive X } \\
\text { Navigation condition X Subjects }\end{array}$ & 49 & 5.5102 & & \\
\hline
\end{tabular}


Table 13. ANOVA's for driver performance measures addressing navigation condition (continued).

\begin{tabular}{|c|c|c|c|c|}
\hline \multicolumn{5}{|l|}{ Speed Variance } \\
\hline Source & $\mathrm{dF}$ & MS & $\mathrm{F}$ & $\mathrm{P}$ \\
\hline Local users' first drive \& Local users' second drive & 1 & 2698.25 & 0.74 & 0.4094 \\
\hline Navigation condition & 5 & 2480.02 & 1.11 & 0.3676 \\
\hline $\begin{array}{l}\text { Local users' first drive \& Local users' second drive } \mathrm{X} \\
\text { Navigation condition }\end{array}$ & 5 & 328.17 & 0.29 & 0.9160 \\
\hline $\begin{array}{l}\text { Local users' first drive \& Local users' second drive X } \\
\text { Subjects }\end{array}$ & 11 & 3667.93 & & \\
\hline Navigation condition X Subjects & 55 & 2241.29 & & \\
\hline $\begin{array}{l}\text { Local users' first drive \& Local users' second drive X } \\
\text { Navigation condition X Subjects }\end{array}$ & 49 & 1130.02 & & \\
\hline \multicolumn{5}{|l|}{ Negative Longitudinal Acceleration } \\
\hline Source & $\mathrm{dF}$ & MS & $\mathrm{F}$ & $\mathrm{P}$ \\
\hline Local users' first drive \& Local users' second drive & 1 & 0.000047 & 0.98 & 0.3433 \\
\hline Navigation condition & 5 & 0.000031 & 0.59 & 0.7079 \\
\hline $\begin{array}{l}\text { Local users' first drive \& Local users' second drive } \mathrm{X} \\
\text { Navigation condition }\end{array}$ & 5 & 0.000014 & 0.28 & 0.9238 \\
\hline $\begin{array}{l}\text { Local users' first drive \& Local users' second drive X } \\
\text { Subjects }\end{array}$ & 11 & 0.000048 & & \\
\hline Navigation condition X Subjects & 55 & 0.000053 & & \\
\hline $\begin{array}{l}\text { Local users' first drive \& Local users' second drive X } \\
\text { Navigation condition X Subjects }\end{array}$ & 49 & 0.000052 & & \\
\hline \multicolumn{5}{|l|}{ Variance in Negative Longitudinal Acceleration } \\
\hline Source & $\mathrm{dF}$ & MS & $\mathrm{F}$ & $\mathrm{P}$ \\
\hline Local users' first drive \& Local users' second drive & 1 & 0.00000002 & 0.01 & 0.9074 \\
\hline Navigation condition & 5 & 0.00000007 & 0.11 & 0.9890 \\
\hline $\begin{array}{l}\text { Local users' first drive \& Local users' second drive } \mathrm{X} \\
\text { Navigation condition }\end{array}$ & 5 & 0.00000061 & 0.90 & 0.4877 \\
\hline $\begin{array}{l}\text { Local users' first drive \& Local users' second drive X } \\
\text { Subjects }\end{array}$ & 11 & 0.00000164 & & \\
\hline Navigation condition X Subjects & 55 & 0.00000066 & & \\
\hline $\begin{array}{l}\text { Local users' first drive \& Local users' second drive X } \\
\text { Navigation condition X Subjects }\end{array}$ & 49 & 0.00000067 & & \\
\hline
\end{tabular}


Table 13. ANOVA's for driver performance measures addressing navigation condition (continued).

\begin{tabular}{|c|c|c|c|c|}
\hline \multicolumn{5}{|c|}{ Number of Brake Applications Corrected by Travel Time } \\
\hline Source & $\mathrm{dF}$ & MS & $\mathrm{F}$ & $P$ \\
\hline Local users' first drive \& Local users' second drive & 1 & 0.0003 & 1.33 & 0.2754 \\
\hline Navigation condition & 5 & 0.0001 & 1.37 & 0.2504 \\
\hline $\begin{array}{l}\text { Local users' first drive \& Local users' second drive } \mathrm{X} \\
\text { Navigation condition }\end{array}$ & 5 & 0.0002 & 3.86 & 0.0053 \\
\hline $\begin{array}{l}\text { Local users' first drive \& Local users' second drive X } \\
\text { Subjects }\end{array}$ & 11 & 0.0002 & & \\
\hline Navigation condition X Subjects & 55 & 0.0001 & & \\
\hline $\begin{array}{l}\text { Local users' first drive \& Local users' second drive X } \\
\text { Navigation condition X Subjects }\end{array}$ & 49 & 0.0017 & & \\
\hline \multicolumn{5}{|l|}{ Number of Steering Reversals Corrected by Travel Time } \\
\hline Source & $\mathrm{dF}$ & MS & $\mathrm{F}$ & $\mathrm{P}$ \\
\hline Local users' first drive \& Local users' second drive & 1 & 0.0082 & 5.72 & 0.0379 \\
\hline Navigation condition & 5 & 0.0011 & 1.83 & 0.1214 \\
\hline $\begin{array}{l}\text { Local users' first drive \& Local users' second drive } \mathrm{X} \\
\text { Navigation condition }\end{array}$ & 5 & 0.0027 & 4.47 & 0.0021 \\
\hline $\begin{array}{l}\text { Local users' first drive \& Local users' second drive X } \\
\text { Subjects }\end{array}$ & 11 & 0.0014 & & \\
\hline Navigation condition X Subjects & 55 & 0.0006 & & \\
\hline $\begin{array}{l}\text { Local users' first drive \& Local users' second drive X } \\
\text { Navigation condition X Subjects }\end{array}$ & 49 & 0.0006 & & \\
\hline \multicolumn{5}{|l|}{ Time for Which the Brake is Pressed } \\
\hline Source & $\mathrm{dF}$ & MS & $\mathrm{F}$ & $\mathrm{P}$ \\
\hline Local users' first drive \& Local users' second drive & 1 & 4.001 & 0.00 & 0.9715 \\
\hline Navigation condition & 5 & 12795.622 & 4.25 & 0.0024 \\
\hline $\begin{array}{l}\text { Local users' first drive \& Local users' second drive } \mathrm{X} \\
\text { Navigation condition }\end{array}$ & 5 & 18144.800 & 2.06 & 0.0880 \\
\hline $\begin{array}{l}\text { Local users' first drive \& Local users' second drive X } \\
\text { Subjects }\end{array}$ & 11 & 2982.761 & & \\
\hline Navigation condition X Subjects & 55 & 3011.107 & & \\
\hline $\begin{array}{l}\text { Local users' first drive \& Local users' second drive X } \\
\text { Navigation condition X Subjects }\end{array}$ & 49 & 1763.237 & & \\
\hline
\end{tabular}




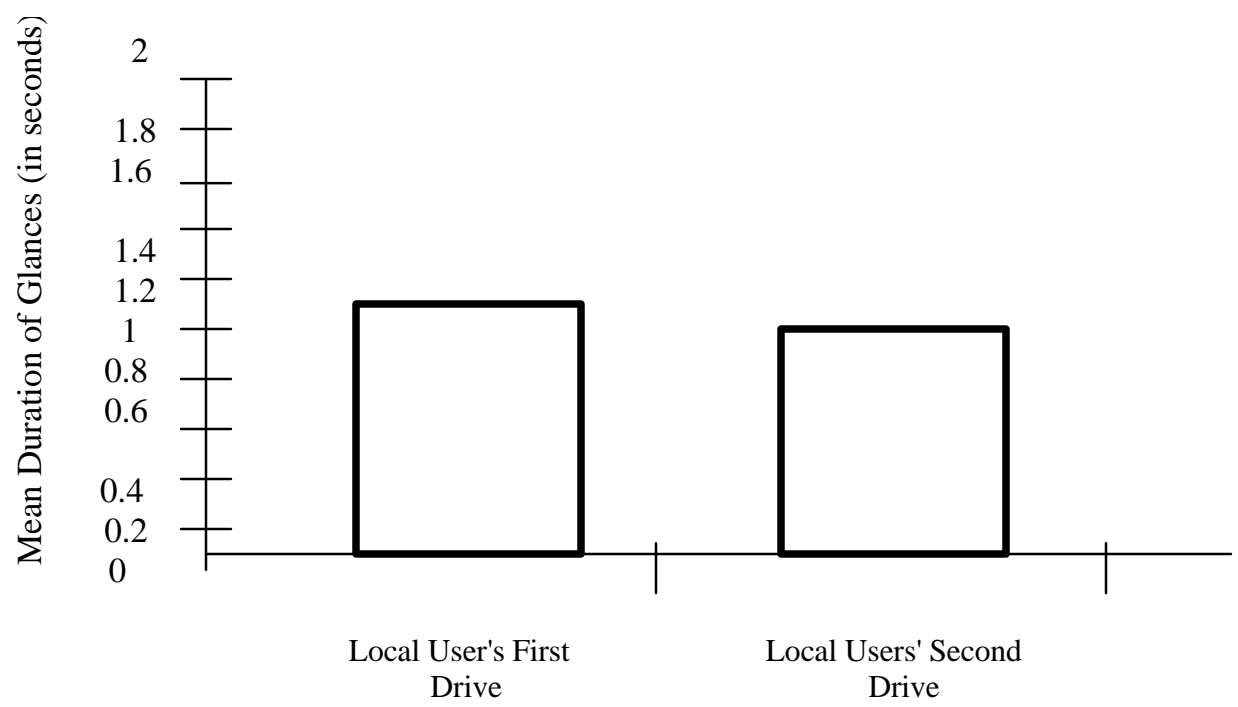

Figure 63. Duration of glances to the navigation information compared across local users' first and second drive.

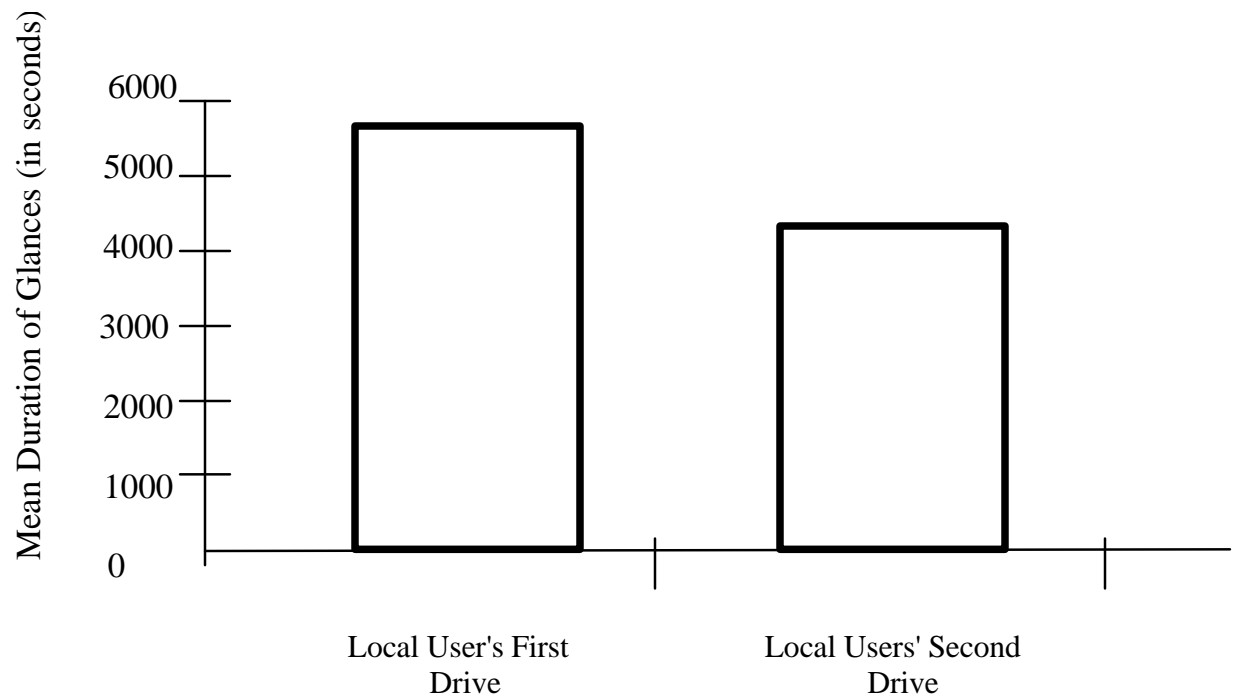

Figure 64. Total number of glances to the navigation information compared across local users' first and second drive.

There were relatively minor changes in mean speed with experience for all of the navigation conditions with the exception of the paper map condition. Given that subjects did not gain experience between runs with paper maps, it is unclear as to why this difference would have occurred. The in-vehicle experimenter noted that on the second drive, subjects planned their paper map route and constructed their hand-written directions more carefully. Therefore, it appears that this difference was due to a practice effect motivated in part by novice driver frustration with their initial map run. 


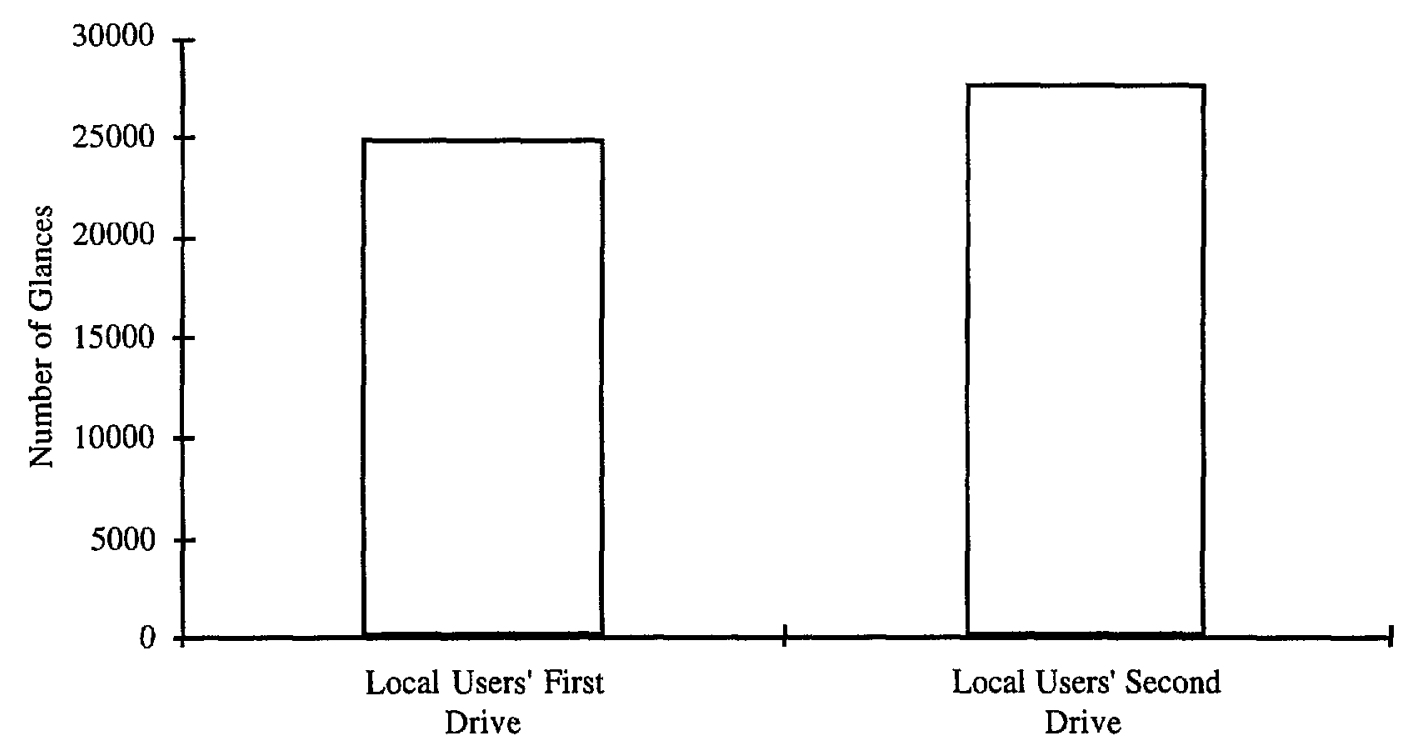

Figure 65. Total number of roadway glances compared across local users' first and second drive.

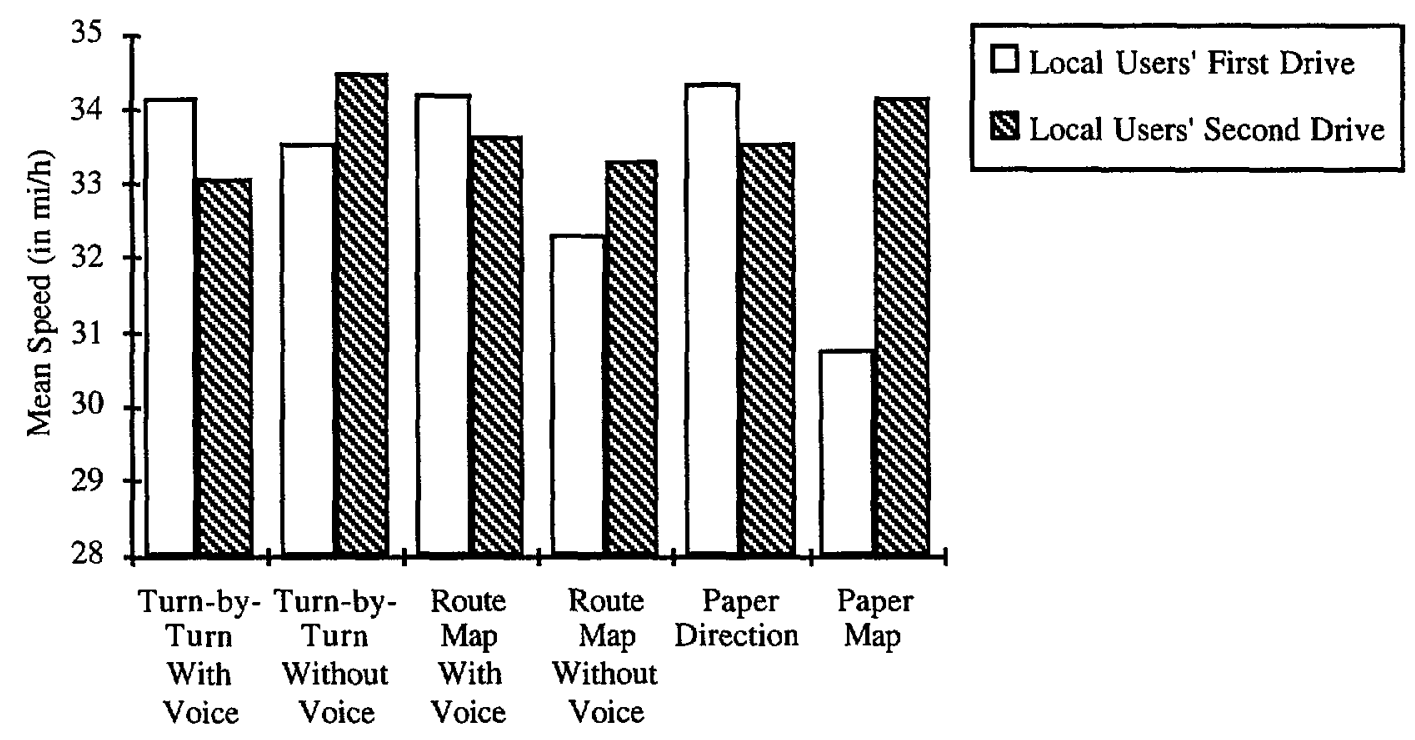

Figure 66. Mean speed for each navigation condition compared across local users' first and second drive. $1 \mathrm{mi} / \mathrm{h}=1.6 \mathrm{~km} / \mathrm{h}$

\section{Steering Reversals}

Figure 67 shows that drivers the number of steering reversals greater than six degrees decreased with increased experience. Steering reversals of this magnitude are indicative of required driver processing resources. The reduction in steering reversals is probably a result of drivers making fewer glances of shorter duration to the navigation system. Because drivers were able to direct their eyes to the roadway more often, they had to make fewer steering corrections to maintain their lane position. 


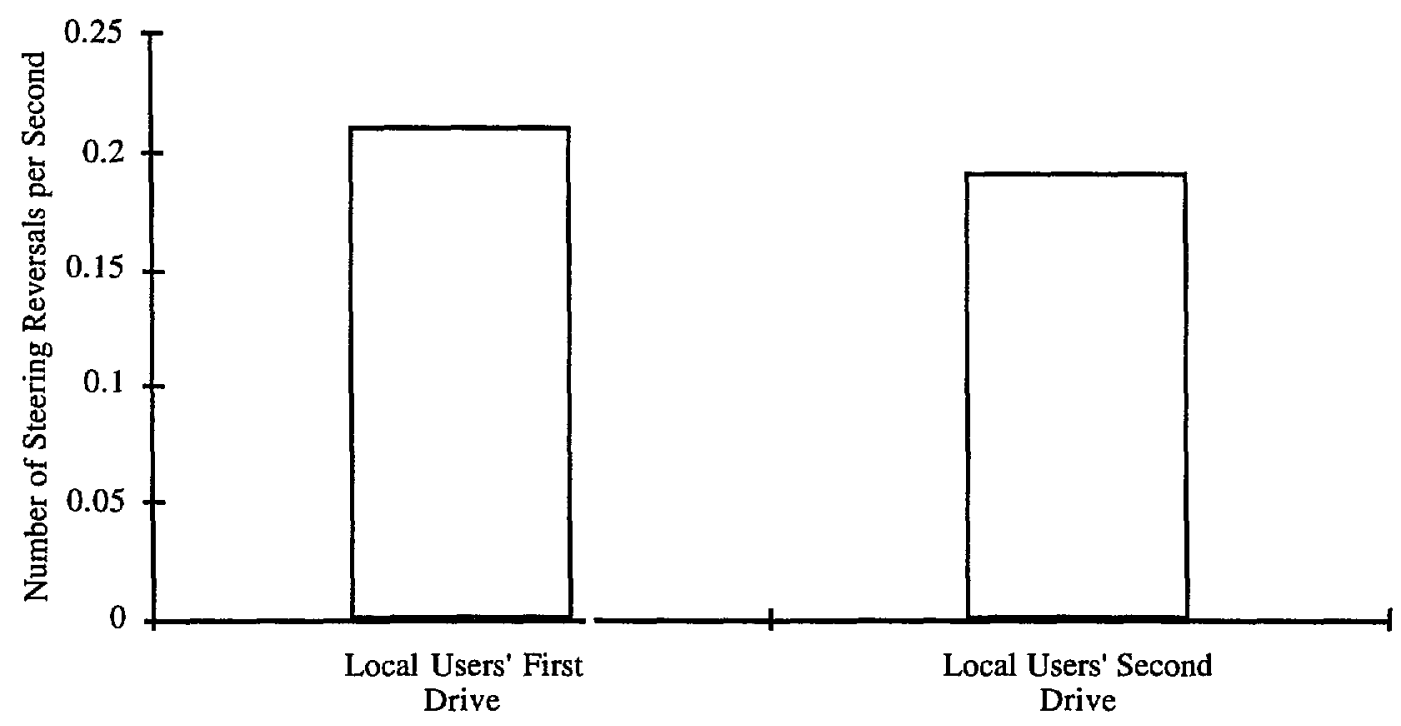

Figure 67. Number of steering reversals over 6 degrees shown per second; compared for local users' first and second drive.

Figure 68 shows the interaction between navigation condition and experience for number of steering reversals. The turn-by-turn with voice, turn-by-turn without voice, route map with voice, and paper map navigation conditions showed a decrease in the number of steering reversals made as experience increased. The route map without voice and paper direction conditions showed small increases in steering reversals as experience increased.

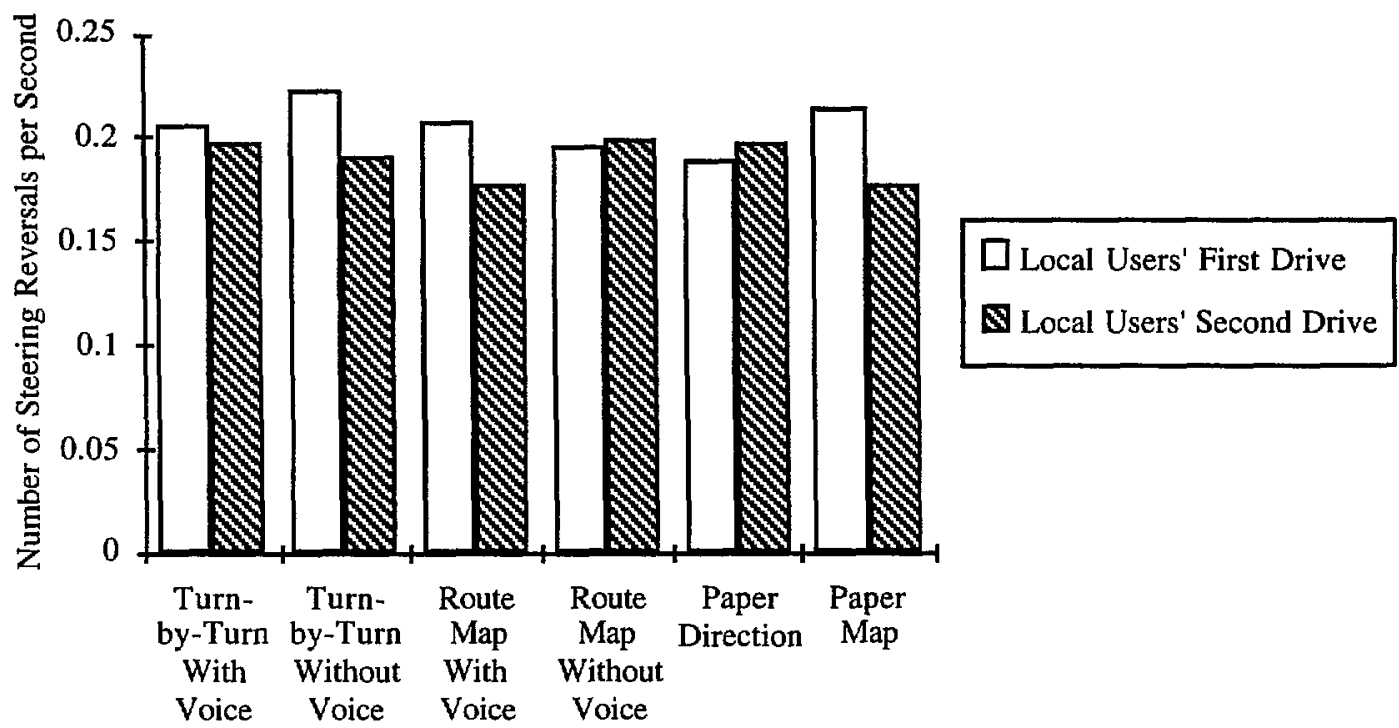

Figure 68. Number of steering reversals over 6 degrees shown per second; compared for each navigation condition and corrected for trip time. 
Figure 69 shows the number of lane deviations that occurred by experience level for each age group. In both age groups the number of lane deviations decreased after drivers had gained more system experience. Figure 70 shows the number of lane deviations that were made by experience level for each navigation condition. With the exception of the route map without voice condition increasing slightly, the number of lane deviations was reduced for each navigation condition as experience was increased. Note that for the experienced users, the number of lane deviations across conditions was largely equivalent. The exception was the paper map control condition, which had fewer lane deviations than the other five navigation conditions.

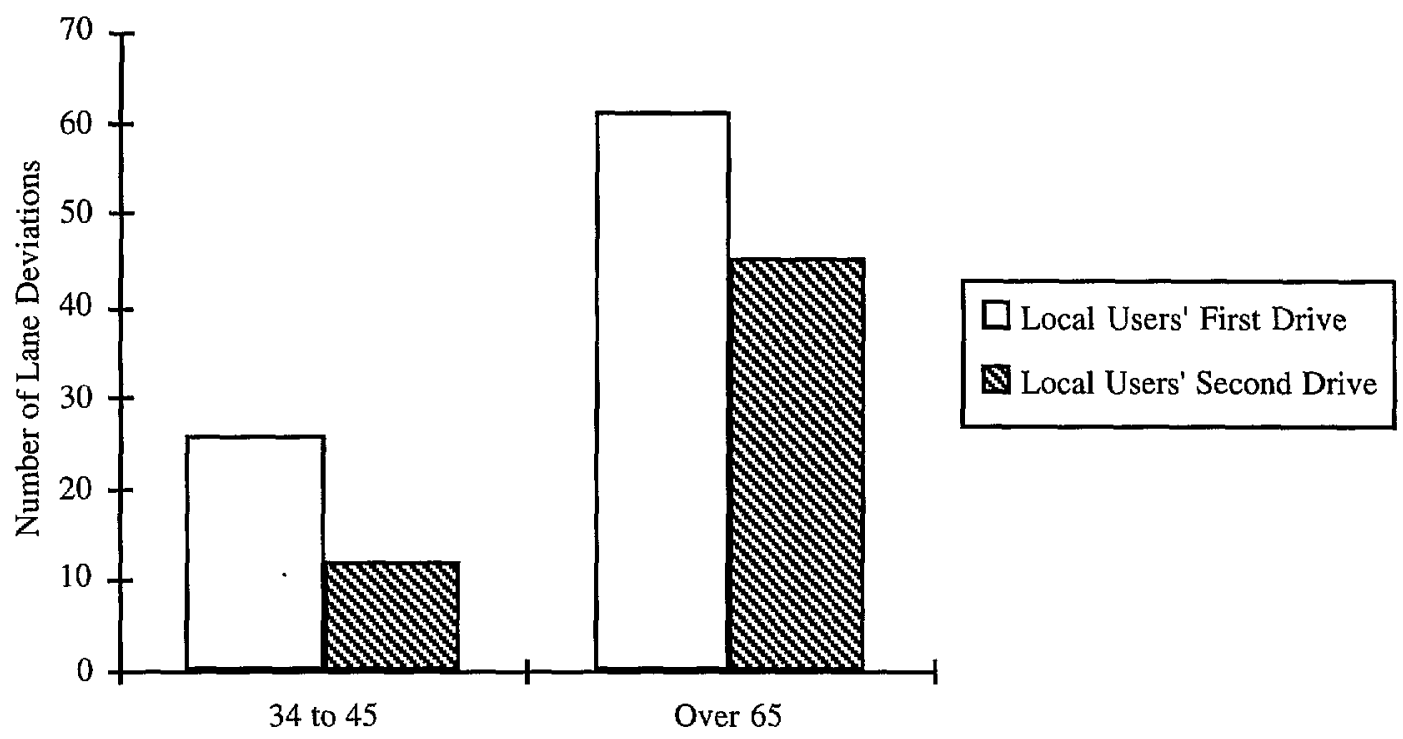

Figure 69. Number of lane deviations for each age group compared across local users' first and second drive.

Another noteworthy result is the relatively high number of lane deviations for novices in the route map with voice condition. Visitors had a higher number of lane deviations in the route map without voice conditions making the sum across users largely equivalent for route map with and without voice. The reason for this apparent difference between types of users and these conditions is not clear.

\section{Brake Applications}

The interaction between navigation condition and experience for number of brake applications corrected for travel time are shown in figure 71 below. The turn-by-turn without voice and paper map conditions showed a decrease in brake applications when drivers had more experience with the system. These conditions also showed higher mean speeds. Given the magnitude of the differences these results may have been due to factors other than experience or navigation condition, such as traffic. 


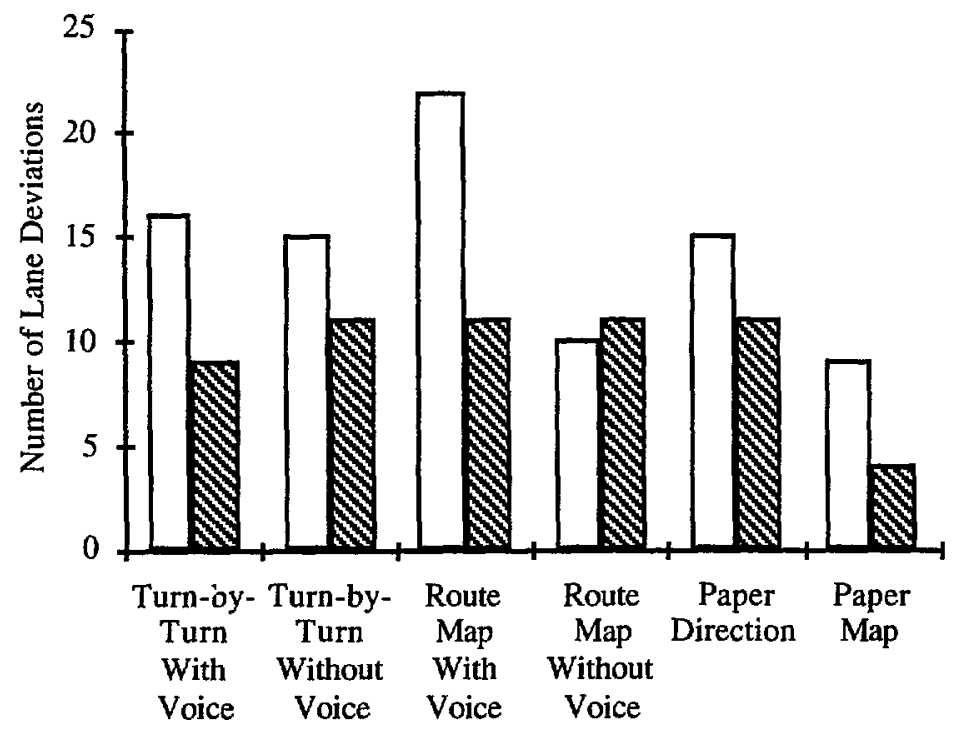

$\square$ Local Users' First Drive

N Local Users' Second Drive

Figure 70. Number of lane deviations for each navigation condition compared across local users' first and second drive.

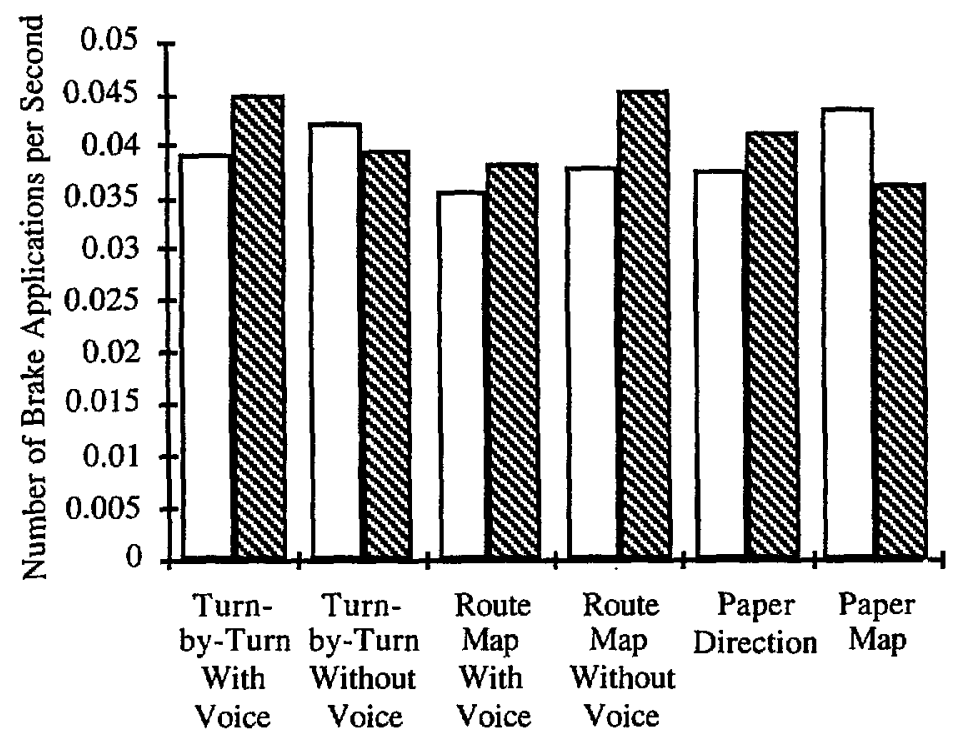

$\square$ Local Users' First Drive

Local Users' Second Drive

Figure 71. Number of brake applications shown per second; compared for each navigation condition and local users' first and second drive. Numbers are corrected for trip time.

\section{Performance Measure Discussion}

The measures of performance that were analyzed to determine the effects of experience on driving performance show that drivers made fewer glances of shorter duration to the navigation aids and drove at higher mean speeds for most navigation conditions after they gained TravTek system experience. In addition, the total number of glances to the roadway environment was greater for experienced drivers than for novice drivers. 
Experience reduced the number and duration of glances to the navigation system, indicating that experienced drivers developed strategies to get system information with less effort than novices. A second hypothesis raised in other studies, is that novice drivers, due to a novelty effect, devote much of their "spare visual capacity" to concentrating on a new and unique system.(12) Thus, experienced drivers look at the display less since the novelty of the system has presumably worn off. In reality, the effect of fewer and shorter glances to the display was probably a combination of these factors.

By making fewer and shorter glances to the system, drivers kept their eyes directed towards the roadway for a greater portion of time as they operated the vehicle. As a result, the amount of steering input required to maintain proper roadway position was reduced, This reduction in visual attention directed towards the navigation aids resulted in generally better driving. This is supported by the fact that experienced drivers made fewer lane deviations than novice drivers and performed more glances of an equivalent duration to roadway-related features.

\section{Measures of Performance for Navigation Performance}

The results of the ANOVA's conducted to analyze navigation performance are shown in tables 14 and 15 below. Time taken to plan the trip and total time taken to plan and drive to the destination were found to be statistically different at the p,0.05 level for contrasts between novice and experienced drivers. An interaction between navigation condition and level of experience was also found statistically significant for time the vehicle was stopped en route to the destination. Other variables, such as the number of stops, the amount of time the vehicle was stopped en route to the destination, and the time required to drive to the destination were not found to be statistically significant.

\section{Time Taken to Plan a Trip}

Figure 72 shows the time required to plan a trip. Experienced drivers took less time to plan their trips than novice drivers. This result indicates that, with experience, drivers became familiar with the layout and functions of the TravTek interface and were subsequently able to reduce their planning times.

\section{Total Time Taken to Plan and Drive to the Destination}

The total time taken to plan and drive to the destination, which includes both planning time and driving time, is shown in figure 73 below. Experienced drivers took less time to plan and drive to their destinations than novice drivers.

\section{Amount of Time the Vehicle Was Stopped While En Route to the Destination}

The interaction between experience and navigation condition for time stopped en route to the destination can be seen in figure 74 below. The route-map without voice navigation condition showed the largest reduction between novice and experienced drivers in the amount of time the vehicle was stopped. The remaining conditions showed relatively minor changes in planning time in both directions. 
Table 14. ANOVA's for navigation performance addressing age.

\begin{tabular}{|c|c|c|c|c|}
\hline \multicolumn{5}{|l|}{ Time Required to Plan a Route } \\
\hline Source & $\mathrm{dF}$ & MS & $\bar{F}$ & $\overline{\mathrm{P}}$ \\
\hline $\begin{array}{l}\text { Local users' first drive \& Local } \\
\text { users' second drive }\end{array}$ & 1 & 42394.943 & 7.99 & 0.0199 \\
\hline Age group & 1 & 88723.262 & 8.01 & 0.0178 \\
\hline $\begin{array}{l}\text { Local users' first drive \& Local } \\
\text { users' second drive x Age group }\end{array}$ & 1 & 6576.935 & 1.24 & 0.2945 \\
\hline Subjects (Age group) & 10 & 11072.026 & & \\
\hline $\begin{array}{l}\text { Local users' first drive \& Local } \\
\text { users' second drive x Subjects } \\
\text { (Age group) }\end{array}$ & 9 & 5308.498 & & \\
\hline \multicolumn{5}{|c|}{ Time Required to Drive to a Destination } \\
\hline Source & $\underline{\mathrm{df}}$ & $\underline{\mathrm{MS}}$ & $\underline{F}$ & $\underline{P}$ \\
\hline $\begin{array}{l}\text { Local users' first drive \& Local } \\
\text { users' second drive }\end{array}$ & 1 & 309081.271 & 2.95 & $0 . \overline{1202}$ \\
\hline Age group & 1 & 282862.877 & 1.28 & 0.2848 \\
\hline $\begin{array}{l}\text { Local users' first drive \& Local } \\
\text { users' second drive x Age group }\end{array}$ & 1 & 14793.615 & 0.14 & 0.7160 \\
\hline Subjects (Age group) & 10 & 221483.810 & & \\
\hline $\begin{array}{l}\text { Local users' first drive \& Local } \\
\text { users' second drive x Subjects } \\
\text { (Age group) }\end{array}$ & 9 & 104918.520 & & \\
\hline
\end{tabular}

Time Required to Plan and Drive to a Destination

\begin{tabular}{|c|c|c|c|c|}
\hline Source & $\mathrm{df}$ & MS & $\overline{\mathrm{F}}$ & \\
\hline $\begin{array}{l}\text { Local users' first drive \& Local } \\
\text { users' second drive }\end{array}$ & 1 & 580381.425 & $\overline{5.23}$ & $0 . \overline{0480}$ \\
\hline Age group & 1 & 688463.182 & 2.82 & 0.1238 \\
\hline $\begin{array}{l}\text { Local users' first drive \& Local } \\
\text { users' second drive x Age gooup }\end{array}$ & 1 & 41107.713 & .37 & 0.5579 \\
\hline Subjects (Age group) & 10 & 243743.950 & & \\
\hline $\begin{array}{l}\text { Local users' first drive \& Local } \\
\text { users' second drive x Subjects } \\
\text { (Age group) }\end{array}$ & 9 & 110992.610 & & \\
\hline \multicolumn{5}{|l|}{ Number of Stops } \\
\hline Source & $\underline{\mathrm{df}}$ & $\underline{\mathrm{MS}}$ & $\underline{F}$ & $\underline{P}$ \\
\hline $\begin{array}{l}\text { Local users' first drive \& Local } \\
\text { users' second drive }\end{array}$ & 1 & 70.2957 & 2.07 & $0 . \overline{1806}$ \\
\hline Age group & 1 & 0.5945 & 0.01 & 0.9076 \\
\hline $\begin{array}{l}\text { Local users' first drive \& Local } \\
\text { users' second drive x Age group }\end{array}$ & 1 & 3.9542 & 0.12 & 0.7399 \\
\hline Subjects (Age group) & 10 & 41.9406 & & \\
\hline $\begin{array}{l}\text { Local users' first drive \& Local } \\
\text { users second drive x Subjects } \\
\text { (Age group) }\end{array}$ & 10 & 33.9300 & & \\
\hline
\end{tabular}


Table 14. ANOVA's for navigation performance addressing age (continued).

\begin{tabular}{|c|c|c|c|c|}
\hline \multicolumn{5}{|l|}{ Mean Duration of Stops } \\
\hline Source & $\mathrm{dF}$ & $\overline{M S}$ & $\overline{\mathrm{F}}$ & $\overline{\mathrm{P}}$ \\
\hline $\begin{array}{l}\text { Local users' first drive \& Local } \\
\text { users' second drive }\end{array}$ & 1 & 11860.501 & 0.540 & 0.4779 \\
\hline Age group & 1 & 19661.237 & $\overline{0.720}$ & 0.4150 \\
\hline $\begin{array}{l}\text { Local users' first drive \& Local } \\
\text { users' second drive x Age group }\end{array}$ & 4 & 926.951 & 0.040 & 0.8408 \\
\hline Subjects (Age group) & 10 & 27187.746 & & \\
\hline $\begin{array}{l}\text { Local users' first drive \& Local } \\
\text { users' second drive x Subjects } \\
\text { (Age group) }\end{array}$ & & 21822.565 & & \\
\hline
\end{tabular}

Table 15. ANOVA's for navigation performance addressing navigation condition.

\begin{tabular}{|l|r|r|r|c|}
\hline \multicolumn{1}{|c|}{ Time to Plan a Trip } & df & \multicolumn{1}{|c|}{ MS } & \multicolumn{1}{|c|}{ Source } & $\underline{\mathrm{P}}$ \\
\hline $\begin{array}{l}\text { Local users' first drive \& Local } \\
\text { users' second drive }\end{array}$ & 1 & 33600.5096 & 6.84 & 0.0258 \\
\hline Navigation condition & 5 & 1026192.100 & 80.33 & 0.0001 \\
\hline $\begin{array}{l}\text { Local users' first drive \& Local users' } \\
\text { second drive x Navigation condition }\end{array}$ & 5 & 1686.4980 & 0.32 & 0.8970 \\
\hline $\begin{array}{l}\text { Local users' first drive \& Local users' } \\
\text { second drive x Subjects }\end{array}$ & 10 & 4910.5900 & & \\
\hline Navigation condition x Subjects & 55 & 12774.1300 & \\
\hline $\begin{array}{l}\text { Local users' first drive \& Local } \\
\text { users' second drive x Navigation } \\
\text { condition x }\end{array}$ & 46 & 5232.2800 & \\
\hline
\end{tabular}

Time Required to Drive to a Destination

\begin{tabular}{|c|c|c|c|c|}
\hline Source & $\mathrm{dF}$ & $\overline{M S}$ & $\overline{\mathrm{F}}$ & $\mathrm{P}$ \\
\hline $\begin{array}{l}\text { Local users' first drive \& Local } \\
\text { users' second drive }\end{array}$ & 1 & 300419.874 & 3.19 & 0.1046 \\
\hline Navigation condition & 5 & 224443.670 & 3.05 & 0.0167 \\
\hline $\begin{array}{l}\text { Local users' first drive \& Local users' } \\
\text { second drive x Navigation condition }\end{array}$ & 5 & 63615.043 & 0.76 & 0.5798 \\
\hline $\begin{array}{l}\text { Local users' first drive \& Local users' } \\
\text { second drive x Subjects }\end{array}$ & 10 & 94300.720 & & \\
\hline Navigation condition $\mathrm{x}$ Subjects & 55 & 73470.140 & & \\
\hline $\begin{array}{l}\text { Local users' first drive \& Local } \\
\text { users' second drive x Navigation } \\
\text { condition x Subjects }\end{array}$ & 461 & 83168.930 & & \\
\hline
\end{tabular}


Table 15. ANOVA's for navigation performance addressing navigation condition (continued).

\begin{tabular}{|c|c|c|c|c|}
\hline Source & $\mathrm{dF}$ & $\mathrm{MS}$ & $\bar{F}$ & $\bar{P}$ \\
\hline $\begin{array}{l}\text { Local users' first drive \& Local } \\
\text { users' second drive }\end{array}$ & & 534927.165 & 5.60 & 0.0395 \\
\hline Navigation condition & 5 & 1852229.550 & 19.78 & 0.0001 \\
\hline $\begin{array}{l}\text { Local users' first drive \& Local users' } \\
\text { second drive } x \text { Navigation condition }\end{array}$ & 5 & 67701.742 & 0.78 & 0.5727 \\
\hline $\begin{array}{l}\text { Local users' first drive \& Local users' } \\
\text { second drive } x \text { Subjects }\end{array}$ & 10 & 95527.790 & & \\
\hline Navigation condition $\mathrm{x}$ Subjects & 55 & 93635.440 & & \\
\hline $\begin{array}{l}\text { Lncal users' first drive \& Local } \\
\text { users' second drive x Navigation } \\
\text { condition x Subiects }\end{array}$ & 46 & 87345.050 & & \\
\hline \multicolumn{5}{|l|}{ Number of Stops } \\
\hline Source & $\underline{\mathrm{df}}$ & MS & $\underline{E}$ & $\underline{\mathrm{P}}$ \\
\hline $\begin{array}{l}\text { Local users' first drive \& Local users' } \\
\text { second drive }\end{array}$ & 1 & 92.8934 & 2.81 & $0 . \overline{1215}$ \\
\hline Navigation condition & 5 & 70.8603 & 5.22 & 0.0005 \\
\hline $\begin{array}{l}\text { Local users' first drive \& Local users' } \\
\text { second drive x Navigation condition }\end{array}$ & 5 & 17.6249 & 0.89 & 0.4973 \\
\hline $\begin{array}{l}\text { Local users' first drive \& Local users' } \\
\text { second drive x Subjects }\end{array}$ & 11 & 32.9997 & & \\
\hline Navigation condition $\mathrm{x}$ Subjects & 55 & 13.5827 & & \\
\hline $\begin{array}{l}\text { Navigation condition x Local users' } \\
\text { first drive \& Local users' second } \\
\text { drive x Subjects }\end{array}$ & 47 & 19.8735 & & \\
\hline \multicolumn{5}{|l|}{ Mean Duration of Stops } \\
\hline Source & $\mathrm{dF}$ & $\overline{\mathrm{MS}}$ & $\bar{F}$ & $\overline{\mathrm{P}}$ \\
\hline $\begin{array}{l}\text { Local users' first drive \& Local users' } \\
\text { second drive }\end{array}$ & 1 & 20756.56 & 0.91 & 0.3617 \\
\hline Navigation condition & 5 & 60355.56 & 3.62 & 0.0067 \\
\hline $\begin{array}{l}\text { Local users' first drive \& Local users' } \\
\text { second drive x Navigation condition }\end{array}$ & 5 & 44544.11 & 3.97 & 0.0043 \\
\hline $\begin{array}{l}\text { Local users' first drive \& Local users' } \\
\text { second drive x Subjects }\end{array}$ & 11 & 22912.68 & & \\
\hline Navigation condition $\mathrm{x}$ Subjects & 55 & 16671.42 & & \\
\hline $\begin{array}{l}\text { Navigation condition x Local users' } \\
\text { first drive \& Local users' second } \\
\text { drive x Subjects }\end{array}$ & 47 & 11231.13 & & \\
\hline
\end{tabular}

Navigation Condition Main Effects

With the exception of number of steering reversals, brake applications, and the amount of time the vehicle was stopped en route to the destination discussed in the previous section, 
there were no other statistically significant interactions between navigation condition andhe level of local user experience. However, the navigation condition significantly affected the time taken to plan the trip, total time taken to plan and drive to a destination, and time taken to drive to the destination. Similar results were indicated when the data were combined for the novice local users and visitors as well as other data combinations. Therefore, to avoid redundancy, these results will not be described in detail in this section. A complete discussion of these results may be found in the driving task intrusion issue of his report.

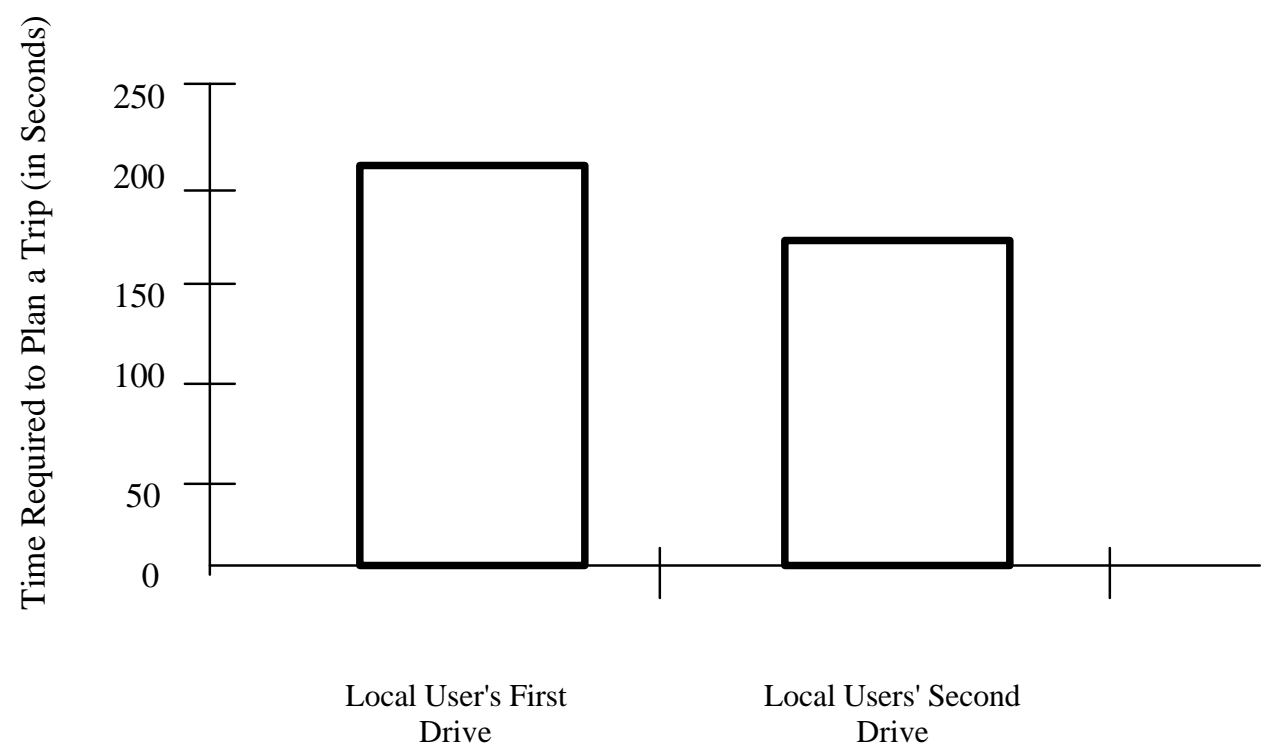

Figure 72. Time required to plan a trip compared across local users' first and second drive.

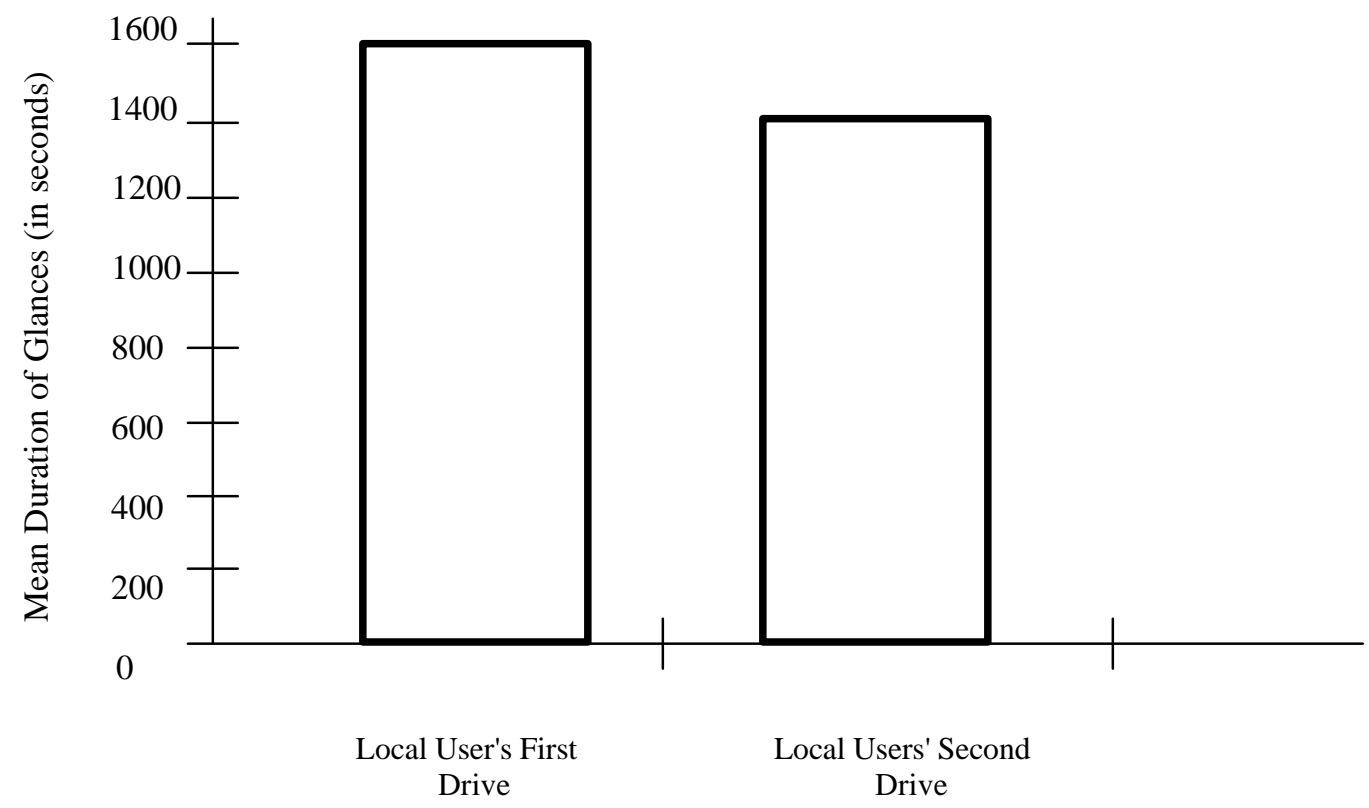

Figure 73. Total time required to plan and drive to the destination compared across local users' first and second drive. 


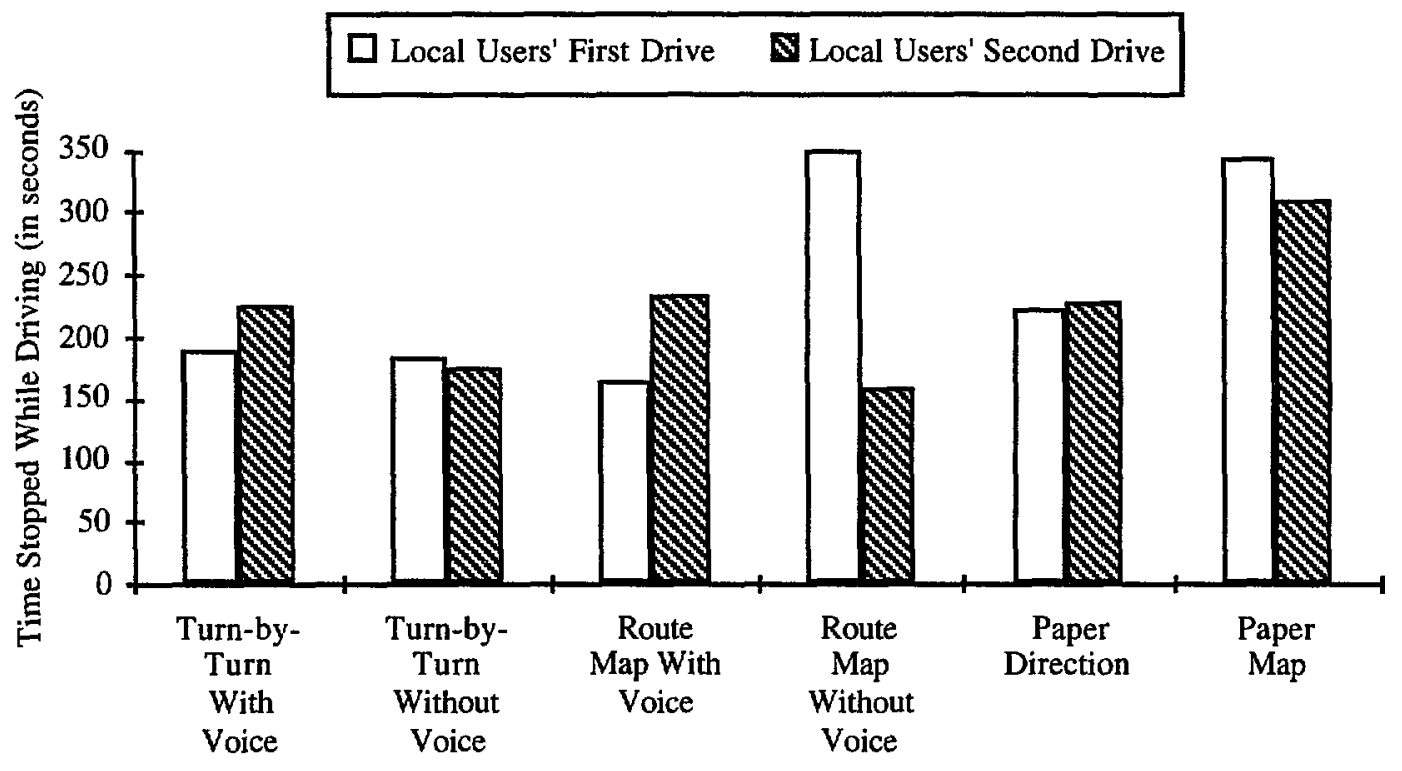

Figure 74. Amount of time the vehicle was stopned while en route to the destination; compared for each navigation condition and local users' first and second drives.

\section{Number of Navigation-Related Errors}

The number of navigation errors committed by novice and experienced drivers is shown in figure 75. Experienced drivers were lost and off route less often and had fewer missed turns. However, with increased experience, there was an increase in the number of indecisions about turn correctness. The reduction in number of missed turns and increase in indecisions about turn correctness between novice and experienced indicates that as drivers began to trust the TravTek navigation information, they made fewer wrong turns. Also, as experience increased, drivers were perhaps indecisive about the turn but trusted the navigation information that TravTek was providing and made the turn anyway. This resulted in the fewer lost and off-route navigation errors.

\section{Discussion For Navigation Performance}

After gaining TravTek system and navigational aid experience, drivers took less time to plan and reach their destinations. This indicates that as drivers became familiar with the system, they also became more proficient at using the navigation system.

The measures of performance for this section indicate that drivers improved navigation performance and spent less time planning trips after gaining system experience. The amount of time that is required to plan a trip is an indicator of how much difficulty the drivers had using the TravTek navigation interface. Another positive indicator of improved navigation performance is the reduction in navigation errors that occurred as experience increased. The more time a driver is lost, off route, or making incorrect turns, the less effective the system is at attaining the goal of improved navigation performance.

Subjects also showed some improvement using the paper map and paper direction list navigation conditions. For the paper map condition, the subjects were able to create their own lists of directions to use while navigating to a destination. After being exposed to the difficulties of navigating with the paper map in the first experimental drive, subjects created 
better lists of directions to aid them in their navigation tasks. This would account for the improvement in this navigation condition even though there was no additional practice using it.

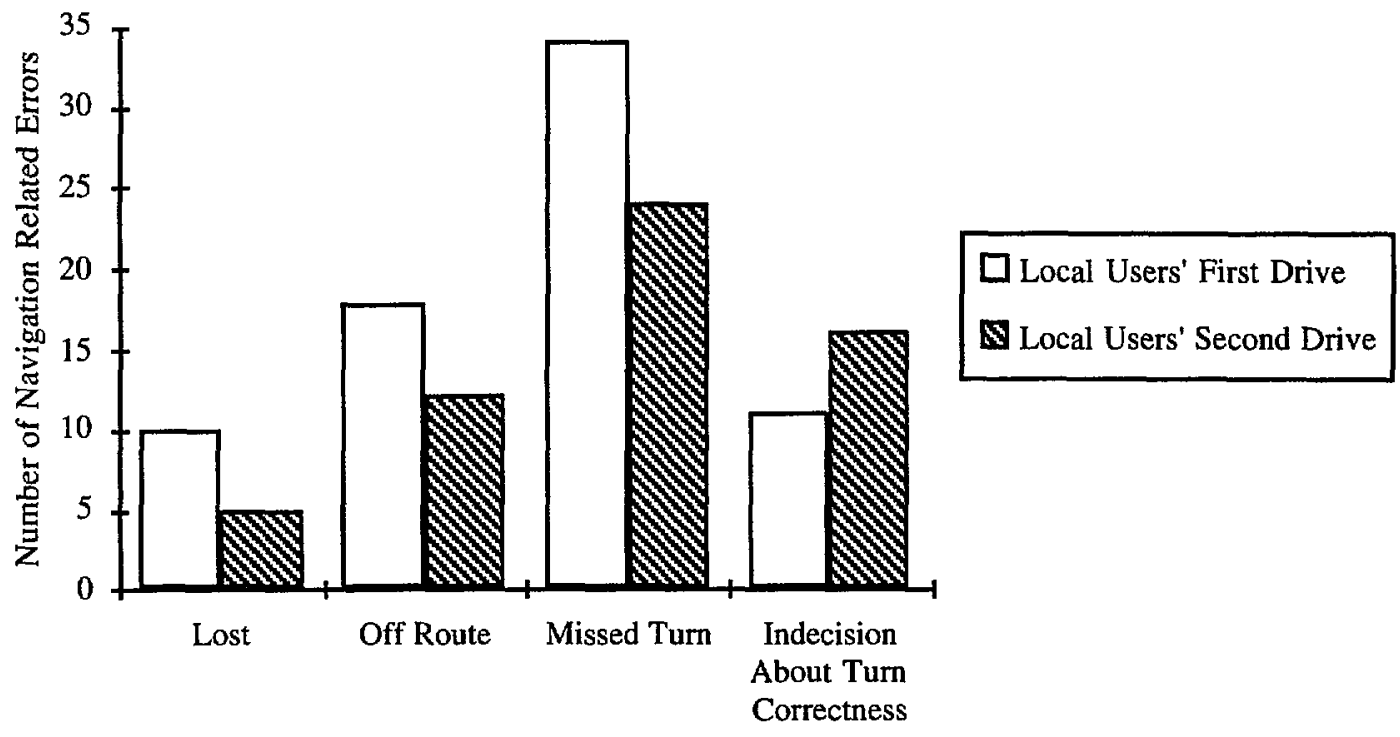

Figure 75. Number of navigation-related errors by type compared across local users' first and second drive.

Experienced drivers also had fewer "serious" navigation-related errors (i.e., being lost and off route) than novice users. This finding further supports that drivers developed a more effective navigation strategy with experience.

\section{Additional Measures of Performance Indicative of Ease of Use}

The results of the ANOVA's that were specifically conducted to analyze usability for user experience are shown in tables 16 and 17 . Note that some of the usability-related variables have also been analyzed in the driving task intrusion section. The main effect of user experience differences measured by subjective workload (shown below), are not statistically different at the $p<0.05$ level. However, there were differences found for the interaction between navigation condition and experience for subjective ratings of time stress, visual effort, and combined workload.

\section{Workload}

Figure 76 shows the mean ratings of time stress that local users assigned to the six navigation conditions after their first and second experimental drives. Figure 77 shows the ratings of visual workload for the same navigation conditions. Figure 78 shows the combined ratings of time stress, visual effort, and psychological stress. For all three variables, the ratings assigned to the turn-by-turn with voice, turn-by-turn without voice, and route map with voice did not change much as experience increased. The mean ratings for route map without voice, paper directions, and paper map conditions increased for experienced drivers. 
Table 16. ANOVA's for performance indicative of ease of use addressing age.

\begin{tabular}{|c|c|c|c|c|}
\hline \multicolumn{5}{|l|}{ Workload Time Stress } \\
\hline Source & $\underline{\mathrm{df}}$ & MS & $\underline{F}$ & $\underline{p}$ \\
\hline $\begin{array}{l}\text { Local users' first drive \& Local } \\
\text { users' second drive }\end{array}$ & 1 & 0.8818 & $\frac{1}{0.75}$ & $\frac{1}{0.4089}$ \\
\hline Age group & 1 & 0.0014 & 0.00 & 0.9806 \\
\hline $\begin{array}{l}\text { Local users' first drive \& Local } \\
\text { users' second drive x Age group }\end{array}$ & 1 & 0.0105 & 0.01 & 0.9267 \\
\hline Subjects (Age group) & 10 & 2.2929 & & \\
\hline $\begin{array}{l}\text { Local users' first drive \& Local } \\
\text { users second drive } x \text { Subjects } \\
\text { (Age group) }\end{array}$ & 9 & 1.1752 & & \\
\hline \multicolumn{5}{|l|}{ Workload Visual Effort } \\
\hline Source & $\underline{\mathrm{df}}$ & $\underline{\text { MS }}$ & $\underline{F}$ & $\underline{\underline{\mathrm{P}}}$ \\
\hline $\begin{array}{l}\text { Local users' first drive \& Local } \\
\text { users' second drive }\end{array}$ & 1 & 0.4581 & 0.53 & $0 . \overline{4863}$ \\
\hline Age group & 1 & 1.3131 & 0.32 & 0.5867 \\
\hline $\begin{array}{l}\text { Local users' first drive \& Local } \\
\text { users' second drive x Age group }\end{array}$ & 1 & 0.0088 & 0.01 & 0.9222 \\
\hline Subjects (Age group) & 10 & 4.1619 & & \\
\hline $\begin{array}{l}\text { Local users' first drive \& Local } \\
\text { users' second drive x Subjects } \\
\text { (Age group) }\end{array}$ & 9 & 0.8692 & & \\
\hline \multicolumn{5}{|l|}{ Workload Psychological Stress } \\
\hline Source & $\underline{\mathrm{df}}$ & $\underline{\underline{\mathrm{MS}}}$ & $\underline{\underline{F}}$ & $\underline{\mathrm{P}}$ \\
\hline $\begin{array}{l}\text { Local users' first drive \& Local } \\
\text { users' second drive }\end{array}$ & 1 & 1.1077 & $\overline{0.33}$ & $0 . \overline{5808}$ \\
\hline Age group & 1 & 0.5116 & 0.11 & 0.7421 \\
\hline $\begin{array}{l}\text { Local users' first drive \& Local } \\
\text { users' second drive x Age group }\end{array}$ & 1 & 0.3828 & 0.11 & 0.7440 \\
\hline Subjects (Age group) & 10 & 4.4683 & & \\
\hline $\begin{array}{l}\text { Local users' first drive \& Local } \\
\text { users' second drive x Subjects } \\
\text { (Age group) }\end{array}$ & 9 & 3.3756 & & \\
\hline \multicolumn{5}{|l|}{ Combined Workload Rating } \\
\hline$\underline{\text { Source }}$ & $\mathrm{df}$ & MS & $\bar{F}$ & $\mathrm{P}$ \\
\hline $\begin{array}{l}\text { Local users' first drive \& Local } \\
\text { users' second drive }\end{array}$ & \begin{tabular}{ll|l}
0 & 1
\end{tabular} & 0.8022 & 0.57 & 0.4710 \\
\hline Age group & 1 & 0.0275 & 0.01 & 0.9249 \\
\hline $\begin{array}{l}\text { Local users' first drive \& Local } \\
\text { users' second drive x Age group }\end{array}$ & & 0.0218 & 0.0 & 0.9040 \\
\hline Subjects (Age group) & & 2.9359 & & \\
\hline $\begin{array}{l}\text { Local users' first drive \& Local } \\
\text { users' second drive x Subjects } \\
\text { (Age group) }\end{array}$ & 9 & 1.4168 & & \\
\hline
\end{tabular}


Table 17. ANOVA's for performance indicative of ease of use addressing navigation condition.

\begin{tabular}{|c|c|c|c|c|}
\hline \multicolumn{5}{|l|}{ Workload Time Stress } \\
\hline$\underline{\text { Source }}$ & $\mathrm{dF}$ & $\mathrm{MS}$ & $\mathrm{F}$ & $\mathrm{P}$ \\
\hline $\begin{array}{l}\text { Local users' first drive \& Local users' } \\
\text { second drive }\end{array}$ & 1 & 1.2803 & 1.13 & 0.3124 \\
\hline Navigation condition & 5 & 3.1794 & 5.33 & 0.0005 \\
\hline $\begin{array}{l}\text { Local users' first drive \& Local users' } \\
\text { second drive x Navigation condition }\end{array}$ & 5 & 0.70001 & 2.57 & 0.0379 \\
\hline $\begin{array}{l}\text { Local users' first drive \& Local users' } \\
\text { second drive x Subjects }\end{array}$ & 10 & 1.1311 & & \\
\hline Navigation condition $\mathrm{x}$ Subjects & 55 & 0.5964 & & \\
\hline $\begin{array}{l}\text { Local users' first drive \& Local users' } \\
\text { second drive x Navigation condition x } \\
\text { Subjects }\end{array}$ & 50 & 0.2720 & & \\
\hline \multicolumn{5}{|l|}{ Workload Visual Effort } \\
\hline Source & $\mathrm{df}$ & $\underline{\underline{\mathrm{MS}}}$ & $\underline{F}$ & $\underline{\mathbf{P}}$ \\
\hline $\begin{array}{l}\text { Local users' first drive \& Local users' } \\
\text { second drive }\end{array}$ & 1 & 0.8000 & 1.00 & $\overline{0 . \overline{3414}}$ \\
\hline Navigation condition & 5 & 7.9469 & 10.92 & 0.0001 \\
\hline $\begin{array}{l}\text { Local users' first drive \& Local users' } \\
\text { second drive x Navigation condition }\end{array}$ & 5 & 0.7814 & 2.50 & 0.0423 \\
\hline $\begin{array}{l}\text { Local users' first drive \& Local users' } \\
\text { second drive x Subjects }\end{array}$ & 10 & 0.8018 & & \\
\hline Navigation condition $\mathrm{x}$ Subjects & 55 & 0.7185 & & \\
\hline $\begin{array}{l}\text { Local users' first drive \& Local users' } \\
\text { second drive x Navigation condition x } \\
\text { Subjects }\end{array}$ & 50 & 0.3120 & & \\
\hline \multicolumn{5}{|l|}{ Workload Psychological Stress } \\
\hline Source & $\underline{\mathrm{df}}$ & $\underline{\mathrm{MS}}$ & $\underline{F}$ & $\underline{\underline{P}}$ \\
\hline $\begin{array}{l}\text { Local users' first drive \& Local users' } \\
\text { second drive }\end{array}$ & 1 & 1.6972 & $\overline{0.51}$ & $0 . \overline{4911}$ \\
\hline Navigation condition & 5 & 4.6964 & 6.27 & 0.0001 \\
\hline $\begin{array}{l}\text { Local users' first drive \& Local users' } \\
\text { second drive } x \text { Navigation condition }\end{array}$ & 5 & 0.6615 & 1.87 & 0.1163 \\
\hline $\begin{array}{l}\text { Local users' first drive \& Local users" } \\
\text { second drive x Subjects }\end{array}$ & 10 & 3.3226 & & \\
\hline Navigation condition $\mathrm{x}$ Subjects & 55 & 0.7484 & & \\
\hline $\begin{array}{l}\text { Local users' first drive \& Local users' } \\
\text { second drive x Navigation condition x } \\
\text { Subjects }\end{array}$ & 501 & $0.3537 \mathrm{I}$ & & \\
\hline
\end{tabular}


Table 17. ANOVA's for performance indicative of ease of use addressing navigation condition (continued).

\begin{tabular}{|c|c|c|c|c|}
\hline \multicolumn{5}{|l|}{ Combined Workload } \\
\hline Source & $\mathrm{dF}$ & MS & $\mathrm{F}$ & $\mathrm{P}$ \\
\hline $\begin{array}{l}\text { Local users' first drive \& Local users' } \\
\text { second drive }\end{array}$ & 1 & 1.2776 & 0.90 & 0.3652 \\
\hline Navigation condition & 5 & 5.0776 & $\overline{8.46}$ & $\overline{0.0001}$ \\
\hline $\begin{array}{l}\text { Local users' first drive \& Local users' } \\
\text { second drive x Navigation condition }\end{array}$ & 5 & 0.6034 & 2.52 & 0.0412 \\
\hline $\begin{array}{l}\text { Local users' first drive \& Local users' } \\
\text { second drive x Subjects }\end{array}$ & 10 & 1.4197 & & \\
\hline Navigation condition x Subjects & 55 & 0.6003 & & \\
\hline $\begin{array}{l}\text { Local users' first drive \& Local users' } \\
\text { second drive x Navigation condition } x \\
\text { Subiects }\end{array}$ & 50 & 0.2394 & & \\
\hline
\end{tabular}

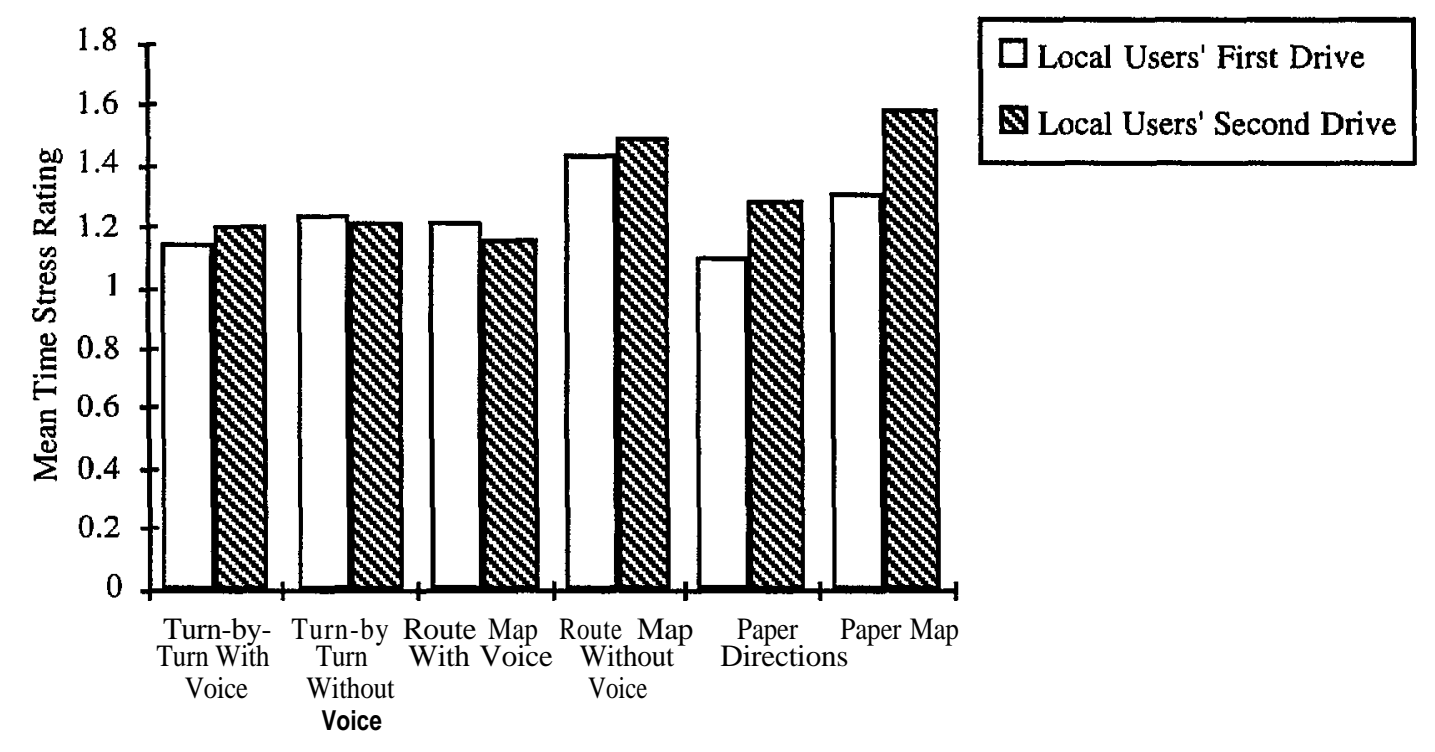

Figure 76. Subjective workload ratings of time stress for each navigation condition compared across local users' first and second drive.

A possible hypothesis for this finding is that before gaining experience, subjects had limited experience with which to rate the navigation conditions relative to one another. After gaining experience, subjects rated the turn-by-turn with voice, turn-by-turn without voice, and route map with voice navigation displays roughly the same in terms of workload. However, based on the ease of using those display conditions, subjects may have felt that the route map without voice, paper directions, and paper map navigation displays caused a higher level of workload. 


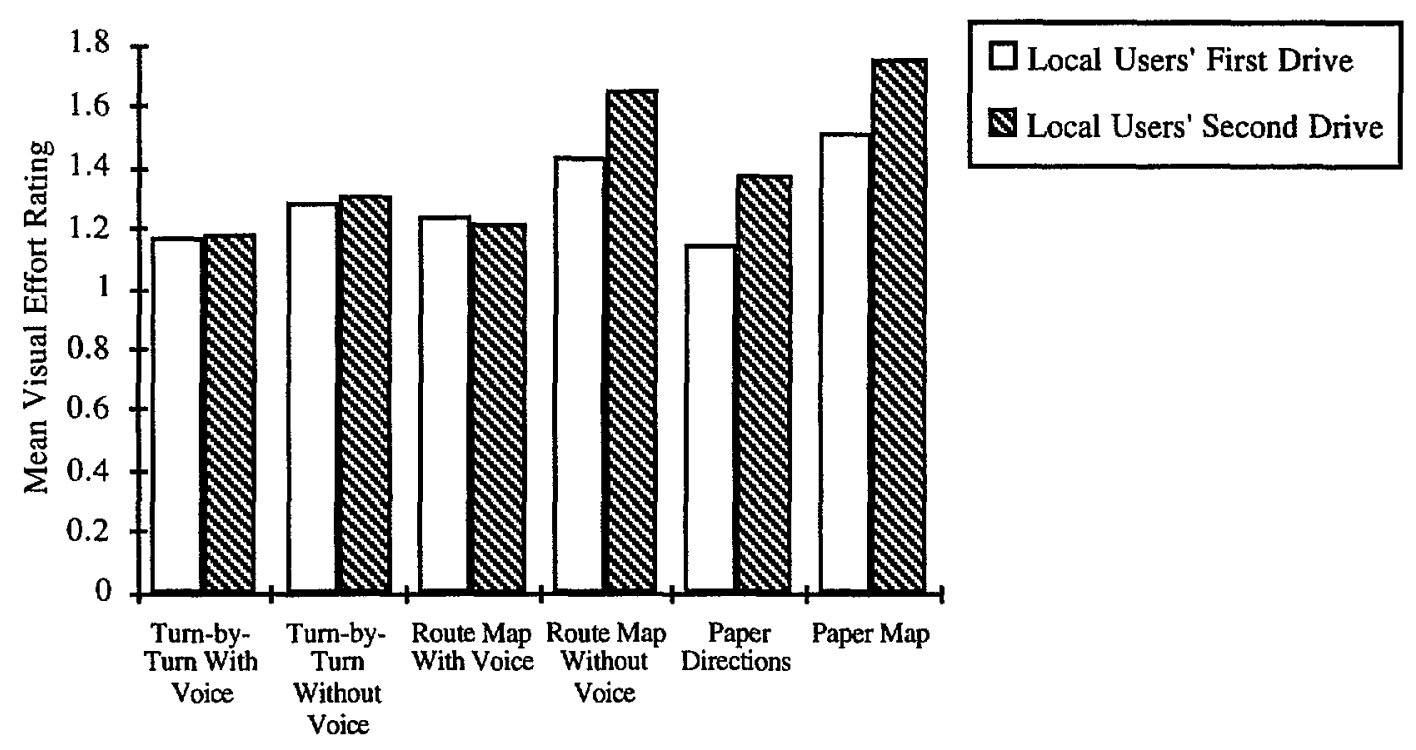

Figure 77. Subjective workload ratings of visual effort for local users' first and second drive.

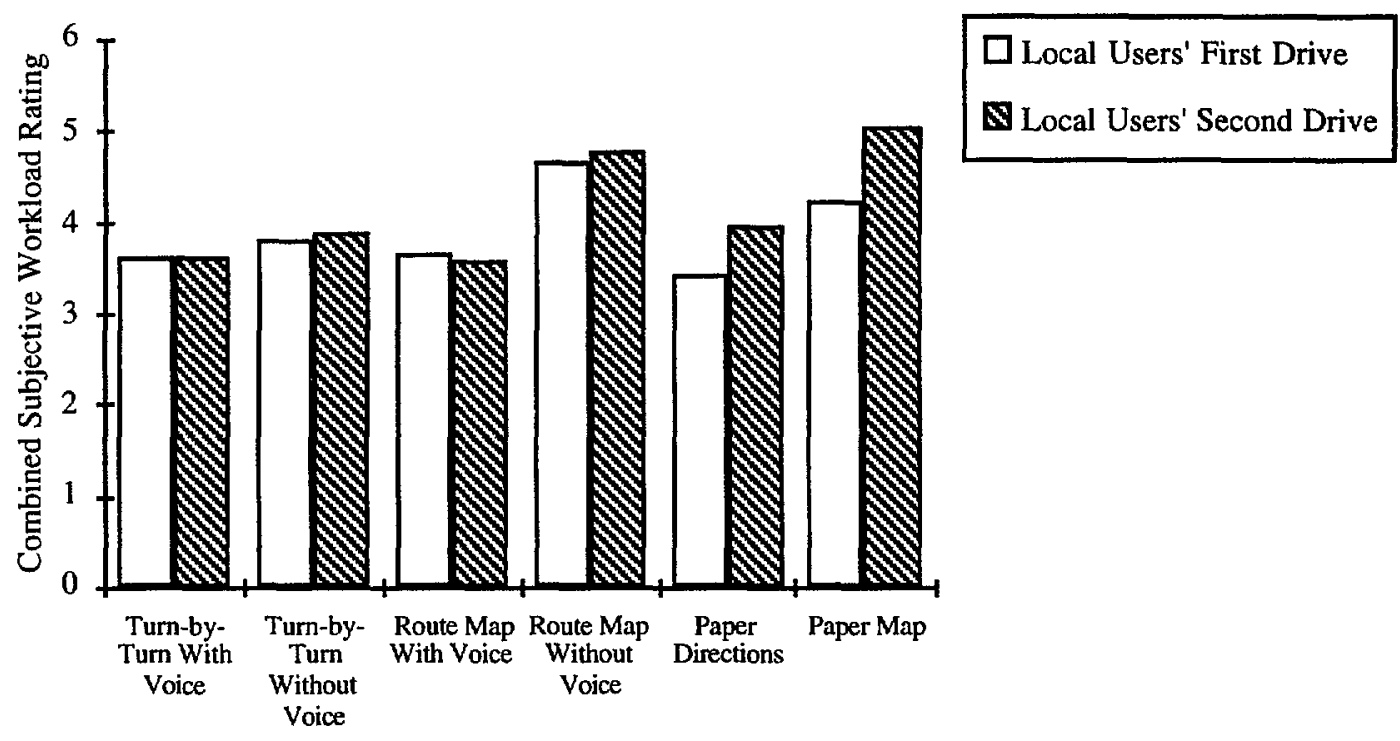

Figure 78. Total of all three subjective workload rating categories for each navigation condition compared across local users' first and second drive.

\section{Location of Glances}

The location of drivers glances were analyzed using a link analysis for novice and experienced users for each navigation condition. The results indicate that, overall, experience had little affect on the percentage of glances to various locations. 
The percentage of glances devoted to looking at each location for the turn-by-turn without voice navigation condition is shown in figures 79 and 80. Figure 79 shows the data for novice drivers and figure 80 shows the data for experienced drivers. Small differences exist between novice and experienced drivers for percentage of glances devoted to the navigation display. Novice drivers directed 24 percent of their glances to the navigation display as compared to 19 percent for experienced drivers.

The route map with voice navigation condition also showed small differences in percentages of glances directed toward the navigation display between novice and experienced drivers.

The data for the route map with voice navigation condition are shown in figures 81 and 82 below. The novice drivers (see figure 81 ) directed 17 percent of their glances to the navigation display compared to 12 percent for experienced drivers (see figure 82).

\section{Discussion For Usability}

The subjective ratings of workload did not show main effect differences as experience increased. However, the workload ratings for the route map without voice, paper direction, and paper map conditions did show an increase as experience increased, which resulted in an interaction between experience and navigation condition. One possible explanation for this is that drivers performed their first drive and made subjective ratings without having much experience with the TravTek navigation conditions and, therefore, did not have a subjective "baseline" for the amount of workload required by all the conditions. Perhaps once drivers became aware of the lower workload required by three TravTek navigation conditions which provided some form of turn-by-turn information, they tended to give higher relative ratings to the route map without voice, paper map and paper directions conditions.

As discussed previously, the measure of performance for glance duration decreased as driver experience with the system increased. The total number of glances to the navigation aids also decreased as system experience increased. Fewer glances of shorter duration to the system indicate that drivers were learning to use the system more efficiently and were extracting information from the navigation displays in fewer glances of shorter duration. The reduced number and duration of glances indicate that the drivers found the system easier to use as they gained more experience.

The location of glance link analysis showed that the percentage of time that drivers spent looking at the navigation display was reduced with experience for the turn-by-turn without voice and route map with voice conditions. This shows that as drivers gained experience using these two types of navigation displays, the percentage of time that was spent looking at the displays decreased. This indicates that learning and experience have a greater positive effect on these types of navigation conditions than other types of displays, in terms of the amount of time that a driver must attend to the display. It may also be the case that the "novelty" wore off with experience, and that drivers devoted spare visual capacity to other locations. Overall, the percentage of time spent looking at TravTek navigation conditions did decrease slightly with experience. Conversely, the paper direction and paper map conditions showed slight increases in percentage of glance time as experience increased. This result may be indicative of the lack of novelty and/or practice gained from using the control navigation aids. 


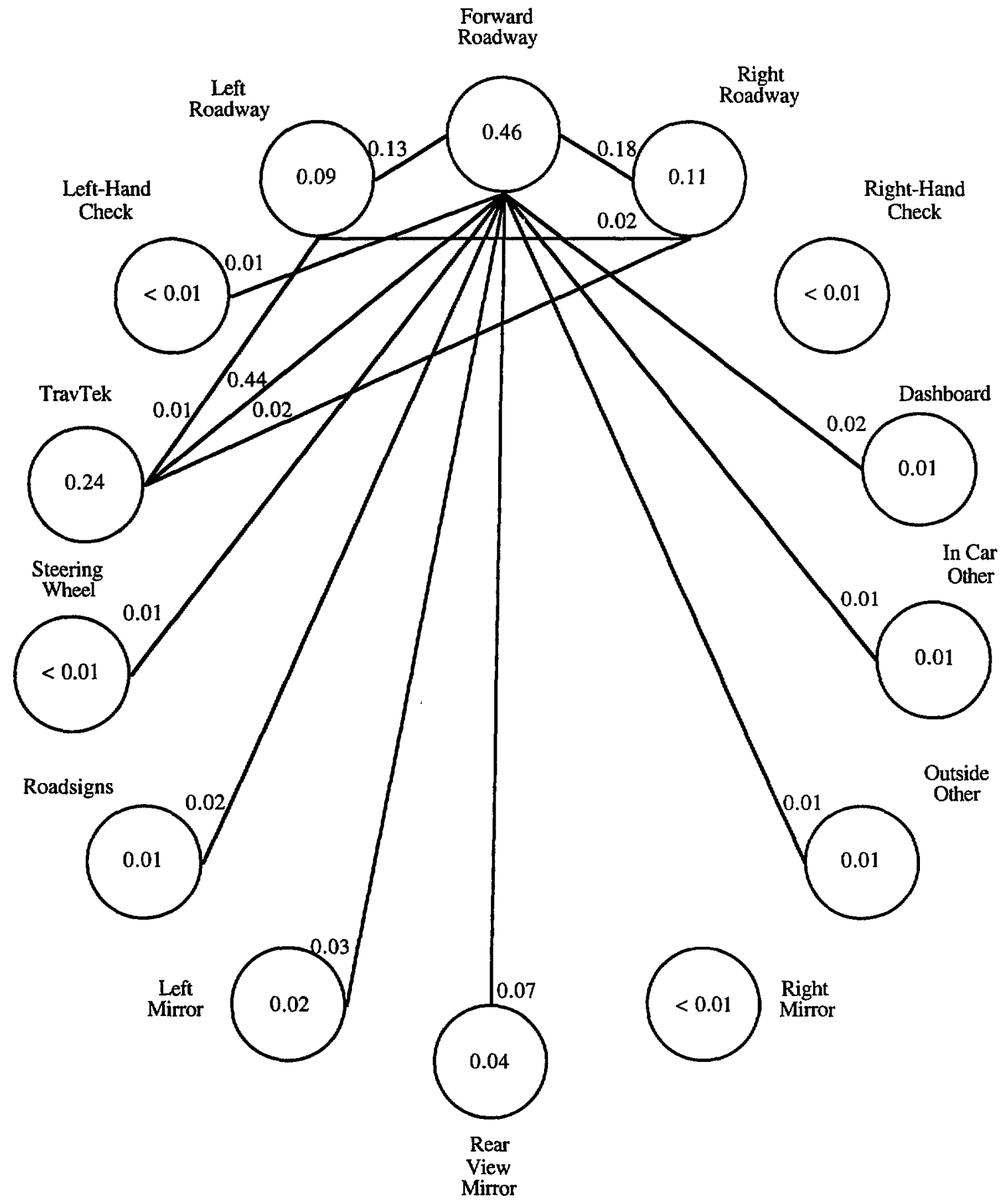

Figure 79. Turn-by-turn without voice link diagram for local users' first drive. 


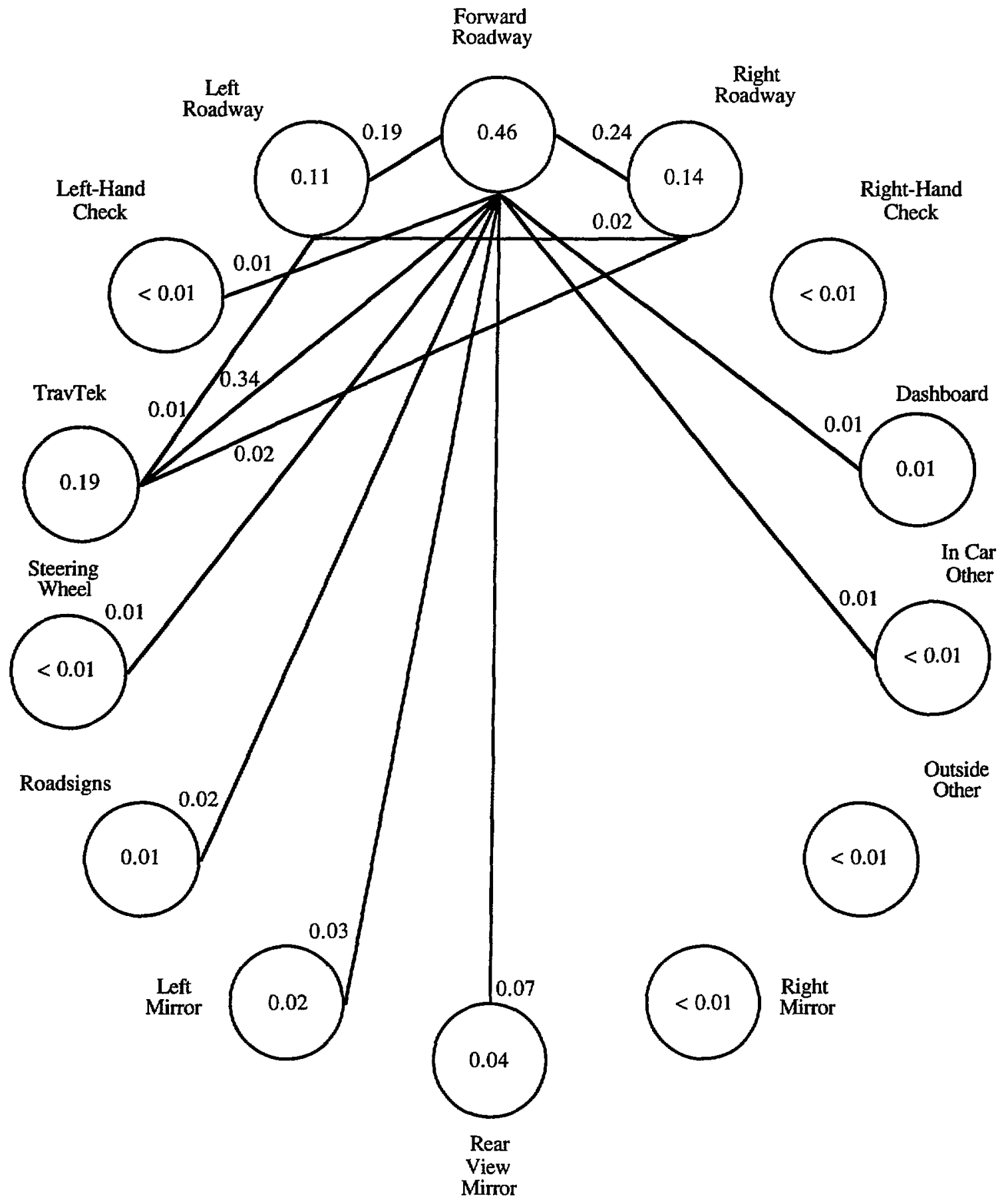

Figure 80. Turn-by-turn without voice link diagram for local users' second drive. 


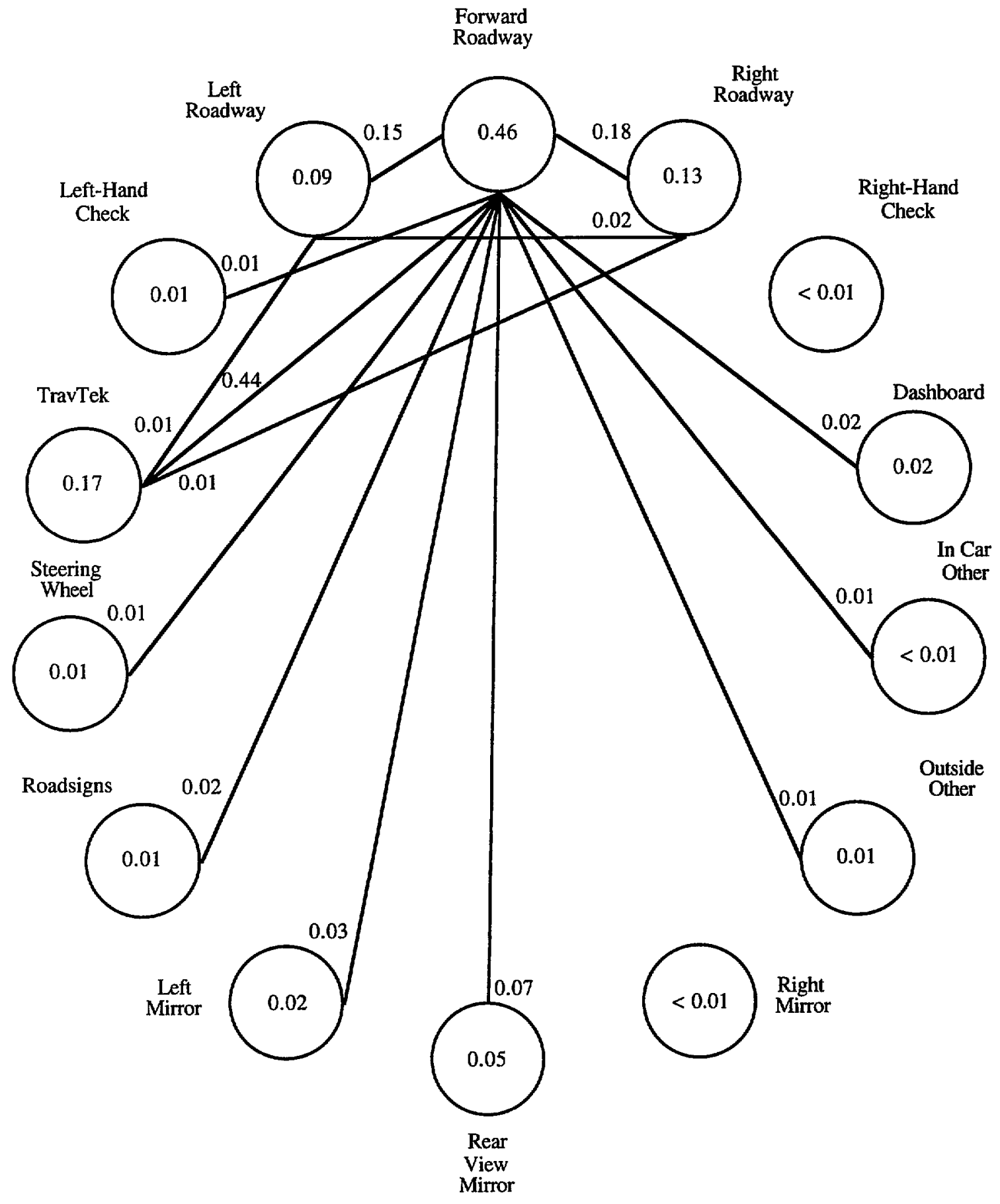

Figure 81. Route map with voice link diagram for local users' first drive. 


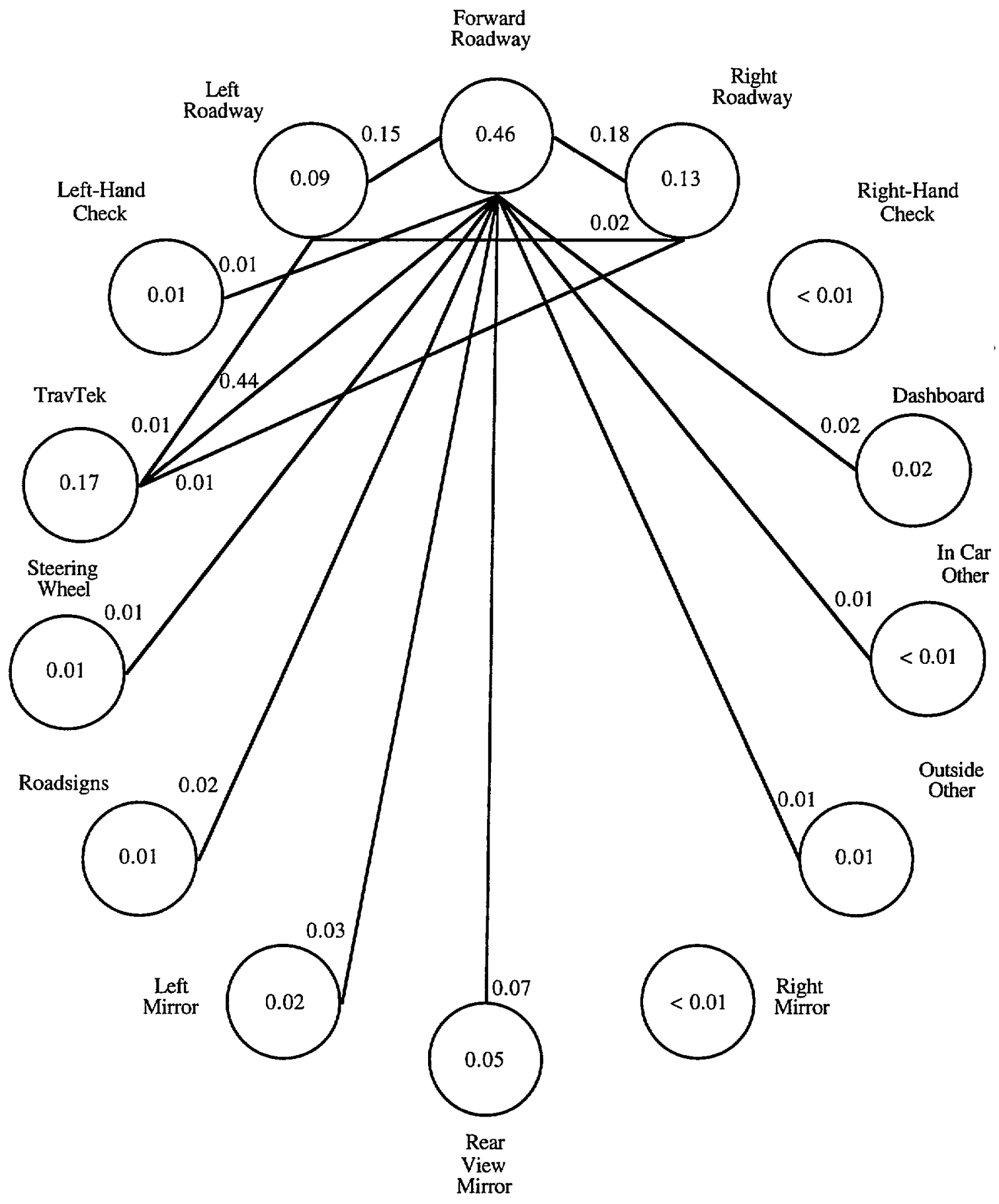

Figure 82. Route map with voice link diagram for local users' second drive.

\section{Measures of Performance for Near Misses and Unsafe Acts}

Safety measures were analyzed to determine the effects of increased experience. The measures that were reviewed included the number of safety-related errors, and number of glances greater than $2.5 \mathrm{~s}$ performed during the experimental drives. All safety analyses performed for this section excluded the minor severity data and the data for driver error with no obstacle present as described in the safety section. 


\section{Number of Safety-Related Errors}

Figure 83 shows the number of safety-related errors by age group and experience. The number of safety-related errors decreased as both age groups gained experience. This is an important finding because the actual number of safety-related errors was reduced to nearly one half for each age group.

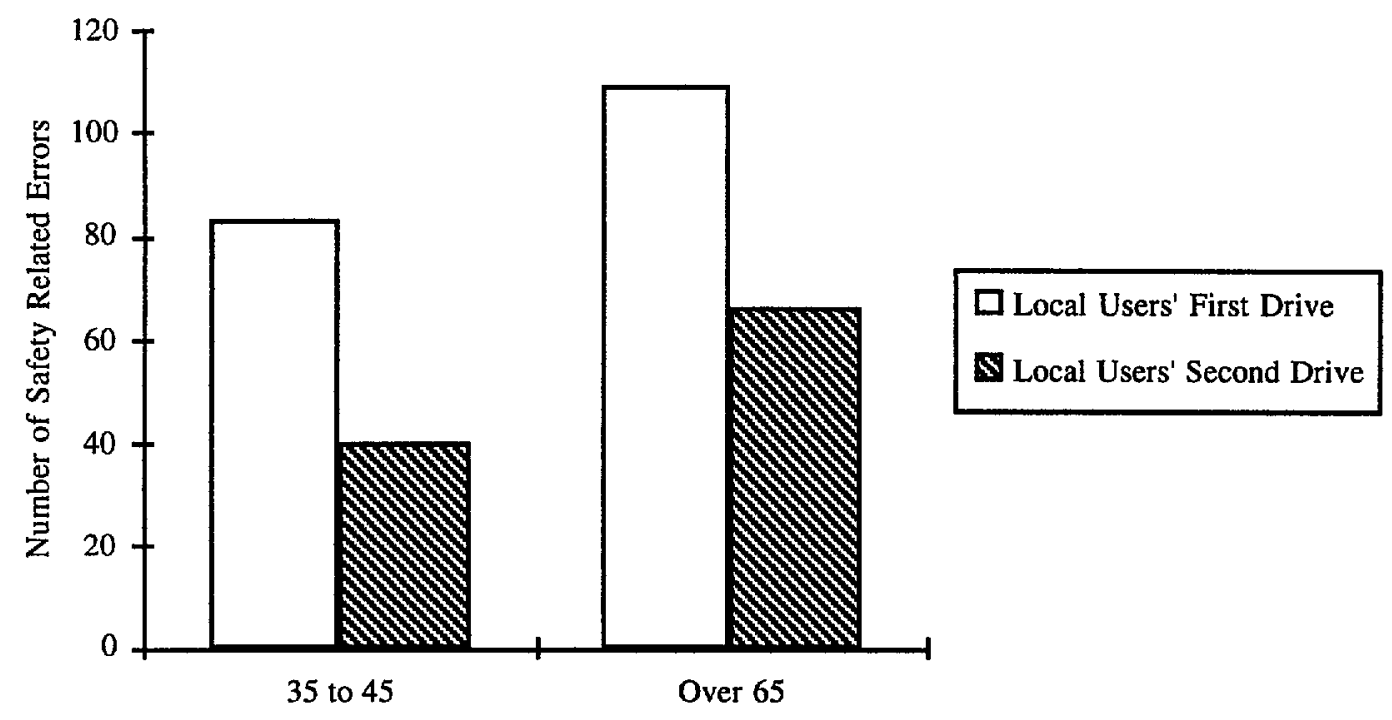

Figure 83. Number of safety-related errors compared across age and local users' first and second drive.

Figure 84 shows that for each navigation condition, the total number of combined safetyrelated errors declined with experience. This shows that safety-related errors were systematically reduced for each TravTek navigation condition. This indicates that with experience, drivers are able to develop strategies for using the TravTek system that improve safe driving practices.

\section{Number of Navigation Glances Greater Than $2.5 \mathrm{~s}$}

Figure 85 shows the number of navigation aid glances greater than $2.5 \mathrm{~s}$ performed during the local users novice and experienced experimental drives. Except in the case of paper directions which showed a small increase, experienced drivers had fewer glances greater than $2.5 \mathrm{~s}$ than novice drivers for all navigation conditions.

\section{Discussion of Near Misses and Unsafe Acts}

The performance measures showed that as experience increased, fewer near-miss and unsafe acts occurred. The number of lane deviations decreased as experience increased. A decrease in the number of lane deviations reduced the potential for the vehicle to collide with opposing traffic or with obstructions along the side of the roadway. As discussed previously, the result of increased experience was the reduction of the amount of visual attention required by the TravTek navigation system. The results for local user experience also support this finding since experienced local users had more roadway glances. 


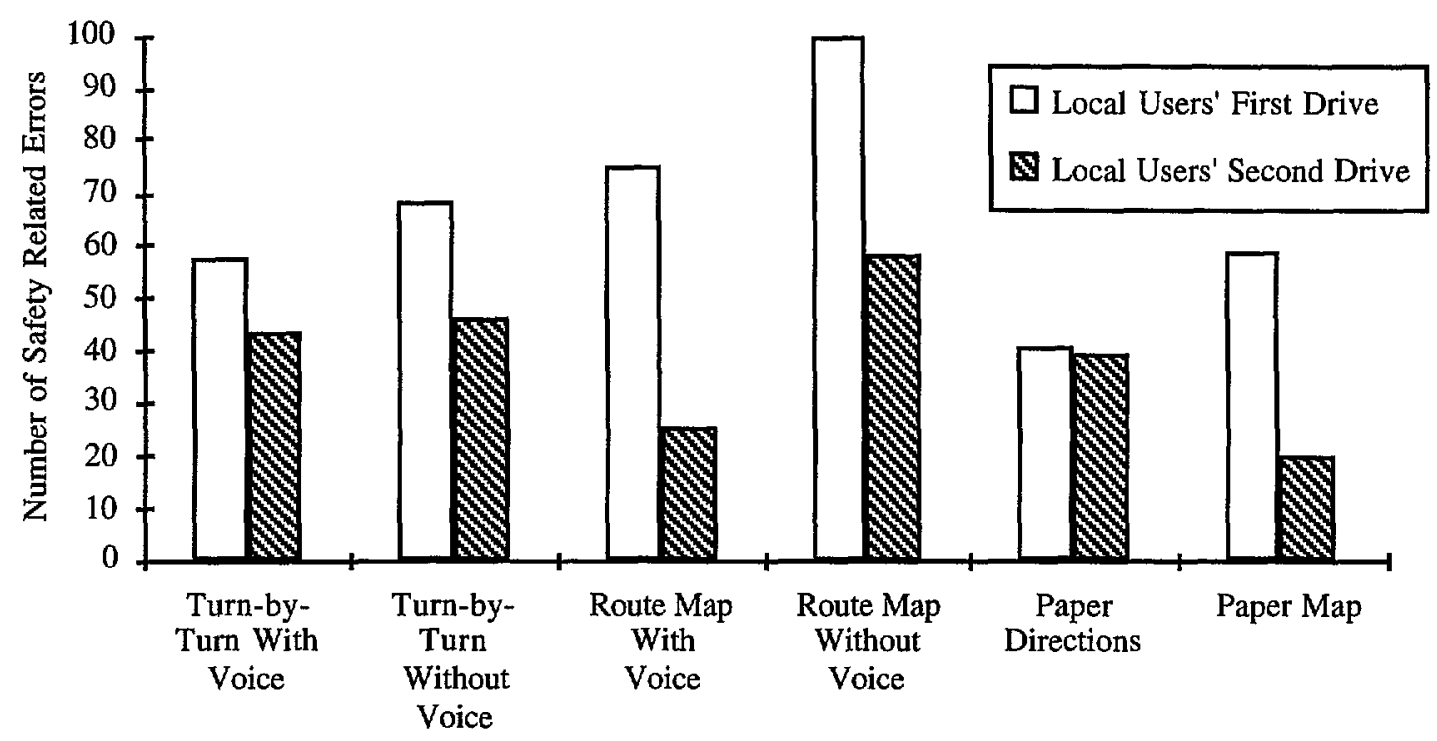

Figure 84. Number of safety-related errors for each navigation condition compared across local users' first and second drive.

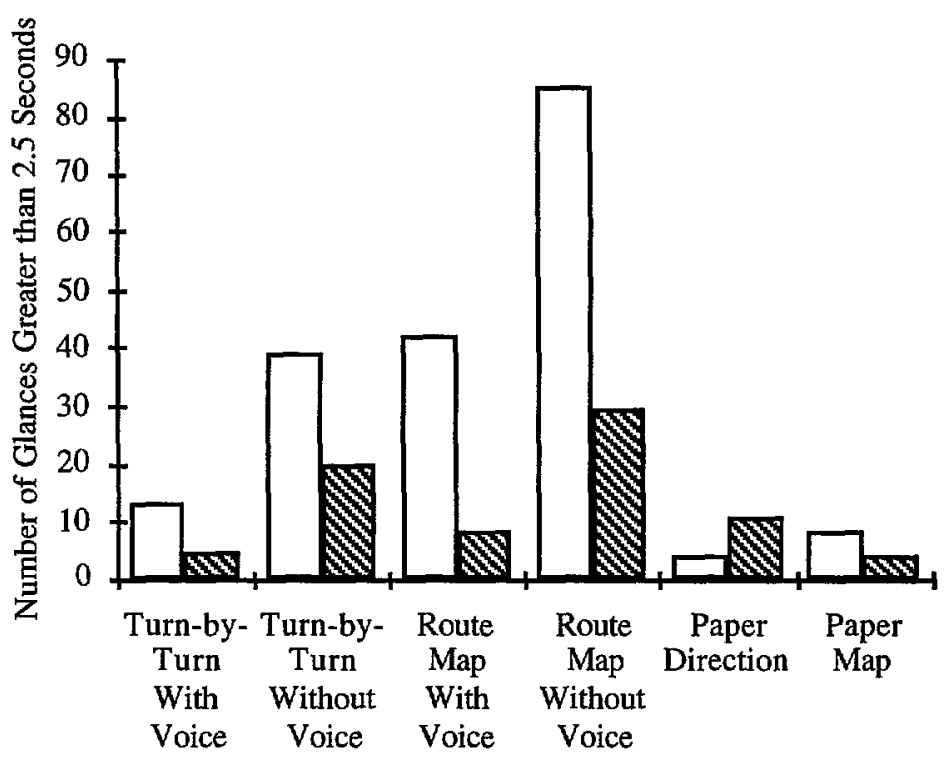

Local Users' First Drive

Local Users' Second Drive

Figure 85. Number of glances greater than $2.5 \mathrm{~s}$ to the navigation display compared across local users' first and second drive.

The results also indicate that as drivers became more familiar with the TravTek system, they committed fewer safety errors overall. The total number of safety-related errors decreased for each individual navigation condition with experience. This could be a result of decreased attention to a navigation aid as drivers developed glance strategies and expectations for the required frequency of glances to the system. It could also be due to a reduction in system novelty for experienced users. In any event, it is clear that safety improved with user experience. 
The number of navigation glances with durations longer than $2.5 \mathrm{~s}$ was reduced as experience was increased. Glances longer than $2.5 \mathrm{~s}$ are indicative of high attention demand. The reduction in longer glances might be due to drivers being able to extract information more efficiently from TravTek and adopt effective strategies for scanning the roadway and navigation displays.

Ultimately, these results support previous conclusions that the more time that the driver has free to scan the roadway environment, the safer the driving task will be. This finding was supported by the safety findings where experienced drivers had fewer safety-related errors. This was particularly true of the route map without voice conditions, which had many more safety-related errors for novice users, but a greatly reduced and comparable number of safety-related errors for experienced local users.

\section{ISSUE 6: DO DRIVING PERFORMANCE, NAVIGATION PERFORMANCE, AND DRIVING SAFETY VARY AS A FUNCTION OF AGE?}

Past research has shown that there are performance, behavioral, and accident rate differences between age groups. To address this in the context of the TravTek system, this issue evaluates the performance of three age groups of visitors (16 to 18,35 to 45 , and $65+$ ) to the Orlando area. Measures obtained in this study are indicative of driving performance, navigation performance, ease of use, and safety.

Two-way ANOVA's were conducted to determine differences between age groups as well as any navigation condition by age group interactions. Since navigation condition main effects have been reported in other sections of this report for combined groups of visitors and local user novices, any navigation condition main effects discovered in the ANOVA's will not be discussed in this section.

\section{Measures of Performance for Driving Performance}

Measures of performance to assess differences in driving performance across age conditions include navigation aid glances, roadway glances, variance in lateral and longitudinal acceleration, steering position variance, velocity mean and variance, number of steering reversals, and brake activation durations.

The ANOVA tables for the driving performance variables are shown in table 18 . Significant differences for age groups were found at the $p<0.05$ level for variance in lateral acceleration, variance in longitudinal acceleration, mean speed, negative longitudinal acceleration, variance in negative longitudinal acceleration and number of large steering reversals. A significant interaction was also found between age and navigation condition for the duration of glances to the navigation aid.

\section{Driver Eye Glance Behavior}

Figure 86 shows the mean duration of navigation aid glances by age group and navigation condition. The oldest subjects (65+) had the longest duration of glances to the navigation aids with the exception of the route map without voice condition, The middle age group (35 to 45) had the shortest glance durations for the paper map and paper direction list conditions. The youngest (16 to 18) subjects had the shortest duration of glances for three of the four TravTek conditions. Note that the youngest and oldest drivers apparently had longer glances to the control conditions relative to several of the TravTek conditions. 
Table 2 1. ANOVA's for driving performance measures addressing driver type and age (continued).

\begin{tabular}{|c|c|c|c|c|}
\hline \multicolumn{5}{|l|}{ Negative Longitudinal Acceleration } \\
\hline Source & $\mathrm{dF}$ & MS & $\mathrm{F}$ & $\mathrm{P}$ \\
\hline Local users' first drive \& Visitors & 1 & 0.00141239 & 3.75 & 0.0671 \\
\hline Age group & 1 & 0.00176797 & 4.69 & 0.0425 \\
\hline Local users' first drive \& Visitors X Age group & 1 & 0.00028397 & 0.75 & 0.3955 \\
\hline Error & 20 & 0.00037665 & & \\
\hline \multicolumn{5}{|l|}{ Variance in Negative Longitudinal Acceleration } \\
\hline Source & $\mathrm{dF}$ & MS & $\mathrm{F}$ & $\mathrm{P}$ \\
\hline Local users' first drive \& Visitors & 1 & 0.00001399 & 2.17 & 0.1565 \\
\hline Age group & 1 & 0.00001844 & 2.86 & 0.1064 \\
\hline Local users' first drive \& Visitors X Age group & 1 & 0.00000068 & 0.10 & 0.7493 \\
\hline Error & 20 & 0.00000645 & & \\
\hline \multicolumn{5}{|c|}{ Number of Brake Applications Corrected by Travel Time } \\
\hline Source & $\mathrm{dF}$ & MS & $\mathrm{F}$ & $\mathrm{P}$ \\
\hline Local users' first drive \& Visitors & 1 & 0.00002145 & 0.07 & 0.8008 \\
\hline Age group & 1 & 0.00066887 & 2.04 & 0.1692 \\
\hline Local users' first drive \& Visitors X Age group & 1 & 0.001033 & 0.03 & 0.8609 \\
\hline Error & 19 & 0.00032744 & & \\
\hline \multicolumn{5}{|c|}{ Number of Steering Reversals Corrected by Travel Time } \\
\hline Source & $\mathrm{dF}$ & $\mathrm{MS}$ & $\mathrm{F}$ & $\mathrm{P}$ \\
\hline Local users' first drive \& Visitors & 1 & 0.01149263 & 2.24 & 0.1509 \\
\hline Age group & 1 & 0.02262839 & 4.41 & 0.0493 \\
\hline Local users' first drive \& Visitors X Age group & 1 & 0.01090937 & 2.13 & 0.1611 \\
\hline Error & 19 & 0.00513102 & & \\
\hline \multicolumn{5}{|l|}{ Time for Which the Brake is Pressed } \\
\hline Source & $\mathrm{dF}$ & MS & $\mathrm{F}$ & $\mathrm{P}$ \\
\hline Local users' first drive \& Visitors & 1 & 4348.33710 & 0.63 & 0.4383 \\
\hline Age group & 1 & 12102.4426 & 1.74 & 0.2023 \\
\hline Local users' first drive \& Visitors X Age group & 1 & 2861.23236 & 0.41 & 0.5284 \\
\hline Error & 19 & 6938.036 & & \\
\hline
\end{tabular}


Table 2 1. ANOVA's for driving performance measures addressing driver type and age (continued).

Duration of Glances to Navigation Aid

Source

Local users' first drive \& Visitors

Navigation condition

Local users' first drive \& Visitors X Navigation condition

Subject (Local users' first drive \& Visitors)

Navigation condition X Subject (Local users' first drive \&

Visitors)

\section{Duration of Roadway Related Glances}

Source

Local users' first drive \& Visitors

Navigation condition

Local users' first drive \& Visitors X Navigation condition

Subject (Local users' first drive \& Visitors)

Navigation condition X Subject (Local users' first drive \&

Visitors)

\begin{tabular}{|r|r|r|r|r|}
\hline & \multicolumn{1}{|c|}{$\mathrm{dF}$} & \multicolumn{1}{|c|}{$\mathrm{MS}$} & $\mathrm{F}$ & $\mathrm{P}$ \\
\hline & 1 & 6.0803 & 0.52 & 0.4792 \\
\hline & 5 & 28.1903 & 9.74 & 0.0001 \\
\hline & 5 & 17.9217 & 6.19 & 0.0001 \\
\hline & 102 & 11.7331 & \multicolumn{3}{|l}{} \\
\hline
\end{tabular}

Variance in Lateral Acceleration

\begin{tabular}{|c|c|c|c|c|}
\hline Source & $\mathrm{dF}$ & MS & $\mathrm{F}$ & $\mathrm{P}$ \\
\hline Local users' first drive \& Visitors & 1 & 0.000018 & 4.25 & 0.0514 \\
\hline Navigation condition & 5 & 0.000001 & 1.13 & 0.3492 \\
\hline Local users' first drive \& Visitors X Navigation condition & 5 & 0.000001 & 1.07 & 0.3816 \\
\hline Subject (Local users' first drive \& Visitors) & 22 & 0.000004 & & \\
\hline $\begin{array}{l}\text { Navigation condition X Subject (Local users' first drive \& } \\
\text { Visitors) }\end{array}$ & 102 & 0.000001 & & \\
\hline
\end{tabular}

Variance in Steering Position

\begin{tabular}{|c|c|c|c|c|}
\hline Source & $\mathrm{dF}$ & MS & $\mathrm{F}$ & $\mathrm{P}$ \\
\hline Local users' first drive \& Visitors & 1 & 377109.725 & 0.64 & 0.4306 \\
\hline Navigation condition & 5 & 79003.82 & 0.78 & 0.5672 \\
\hline Local users' first drive \& Visitors X Navigation condition & 5 & 180568.42 & 1.78 & 0.1234 \\
\hline Subject (Local users' first drive \& Visitors) & 22 & 584827.7 & & \\
\hline $\begin{array}{l}\text { Navigation condition X Subject (Local users' first drive \& } \\
\text { Visitors) }\end{array}$ & 104 & 101454.0 & & \\
\hline
\end{tabular}


Table 22. ANOVA's for driving performance measures addressing navigation condition and driver type.

Variance in Longitudinal Acceleration

Source

Local users' first drive \& Visitors

Navigation condition

Local users' first drive \& Visitors X Navigation condition

Subject (Local users' first drive \& Visitors)

Navigation condition X Subject (Local users' first drive \&

Visitors)

\section{Mean Speed}

Source

Local users' first drive \& Visitors

Navigation condition

Local users' first drive \& Visitors X Navigation condition

Subject (Local users' first drive \& Visitors)

Navigation condition X Subject (Local users' first drive \&

Visitors)

\begin{tabular}{|r|r|l|r|r|}
\hline & $\mathrm{dF}$ & $\mathrm{MS}$ & $\mathrm{F}$ & $\mathrm{P}$ \\
\hline & 1 & 0.000010 & 0.65 & 0.4299 \\
\hline & 5 & 0.000004 & 3.13 & 0.0114 \\
\hline 5 & 0.000001 & 0.92 & 0.4688 \\
\hline & 22 & 0.000016 & \multicolumn{3}{|c|}{} \\
\hline & 104 & 0.000001 & \multicolumn{3}{|c|}{} \\
\hline
\end{tabular}

\section{Speed Variance}

\begin{tabular}{|c|c|c|c|c|}
\hline Source & $\mathrm{dF}$ & MS & $\mathrm{F}$ & $\mathrm{P}$ \\
\hline Local users' first drive \& Visitors & 1 & 6542.8839 & 0.91 & 0.3502 \\
\hline Navigation condition & 5 & 3324.1443 & 1.81 & 0.1174 \\
\hline Local users' first drive \& Visitors X Navigation condition & 5 & 176.4982 & 0.10 & 0.9926 \\
\hline Subject (Local users' first drive \& Visitors) & 22 & 7182.76 & & \\
\hline $\begin{array}{l}\text { Navigation condition X Subject (Local users' first drive \& } \\
\text { Visitors) }\end{array}$ & 104 & 1837.05 & & \\
\hline \multicolumn{5}{|l|}{ Negative Longitudinal Acceleration } \\
\hline Source & $\mathrm{dF}$ & MS & $\mathrm{F}$ & $\mathrm{P}$ \\
\hline Local users' first drive \& Visitors & 1 & 0.001421 & 3.15 & 0.896 \\
\hline Navigation condition & 5 & 0.000032 & 0.82 & 0.5406 \\
\hline Local users' first drive \& Visitors X Navigation condition & 5 & 0.000027 & 0.68 & 0.6381 \\
\hline Subject (Local users' first drive \& Visitors) & 22 & 0.00045 & & \\
\hline $\begin{array}{l}\text { Navigation condition X Subject (Local users' first drive \& } \\
\text { Visitors) }\end{array}$ & 104 & 0.000040 & & \\
\hline
\end{tabular}


Table 22. ANOVA's for driving performance measures addressing navigation condition and driver type (continued).

Variance in Negative Longitudinal Acceleration

Source

Local users' first drive \& Visitors

Navigation condition

Local users' first drive \& Visitors X Navigation condition

Subject (Local users' first drive \& Visitors)

Navigation condition X Subject (Local users' first drive \&

Visitors)

\begin{tabular}{|c|c|c|c|}
\hline $\mathrm{dF}$ & MS & $\mathrm{F}$ & $\mathrm{P}$ \\
\hline 1 & 0.00001413 & 2.05 & 0.1666 \\
\hline 5 & 0.00000012 & 0.19 & 0.9640 \\
\hline 5 & 0.00000014 & 0.23 & 0.9485 \\
\hline 22 & 0.0000069 & & \\
\hline 104 & 0.000062 & & \\
\hline
\end{tabular}

\section{Number of Brake Applications Corrected by Travel Time}

Source

Local users' first drive \& Visitors

Navigation condition

Local users' first drive \& Visitors X Navigation condition

Subject (Local users' first drive \& Visitors)

Navigation condition X Subject (Local users' first drive \&

Visitors)

\begin{tabular}{|c|c|c|c|}
\hline $\mathrm{dF}$ & MS & $\mathrm{F}$ & $\mathrm{P}$ \\
\hline 1 & 0.00004027 & 0.12 & 0.7328 \\
\hline 5 & 0.00006553 & 1.36 & 0.2466 \\
\hline 5 & 0.00006183 & 1.28 & 0.2779 \\
\hline 21 & 0.00033648 & & \\
\hline 101 & 0.00004826 & & \\
\hline
\end{tabular}

Number of Steering Reversals Corrected by Travel Time

\begin{tabular}{|c|c|c|c|c|}
\hline Source & $\mathrm{dF}$ & MS & $\mathrm{F}$ & $\mathrm{P}$ \\
\hline Local users' first drive \& Visitors & 1 & 0.01155617 & 1.82 & 0.1919 \\
\hline Navigation condition & 5 & 0.00190767 & 2.85 & 0.0188 \\
\hline Local users' first drive \& Visitors X Navigation condition & 5 & 0.00096716 & 1.45 & 0.2143 \\
\hline Subject (Local users' first drive \& Visitors) & 21 & 0.00635476 & & \\
\hline $\begin{array}{l}\text { Navigation condition X Subject (Local users' first drive \& } \\
\text { Visitors) }\end{array}$ & 101 & 0.00066880 & & \\
\hline
\end{tabular}

Time for Which the Brake is Pressed

\begin{tabular}{|c|c|c|c|c|}
\hline Source & $\mathrm{dF}$ & MS & $\mathrm{F}$ & $\mathrm{P}$ \\
\hline Local users' first drive \& Visitors & 1 & 4402.0129 & 0.62 & 0.4414 \\
\hline Navigation condition & 5 & 19470.4572 & 6.27 & 0.0001 \\
\hline Local users' first drive \& Visitors X Navigation condition & 5 & 2016.3384 & 0.65 & 0.6630 \\
\hline Subject (Local users' first drive \& Visitors) & 22 & 7148.313 & & \\
\hline $\begin{array}{l}\text { Navigation condition X Subject (Local users' first drive \& } \\
\text { Visitors) }\end{array}$ & 101 & 3107.504 & & \\
\hline
\end{tabular}




\section{Driver Eye Glance Behavior}

The number of navigation-aid glances for visitor and local user first drive appears in figurd 21. As shown, visitors had a larger number of glances to the navigation aids overall, thanthe local users. This result is intuitive in that visitors probably glanced more at thenavigation aids because they were unfamiliar with the area and needed more currentlocation and route information.

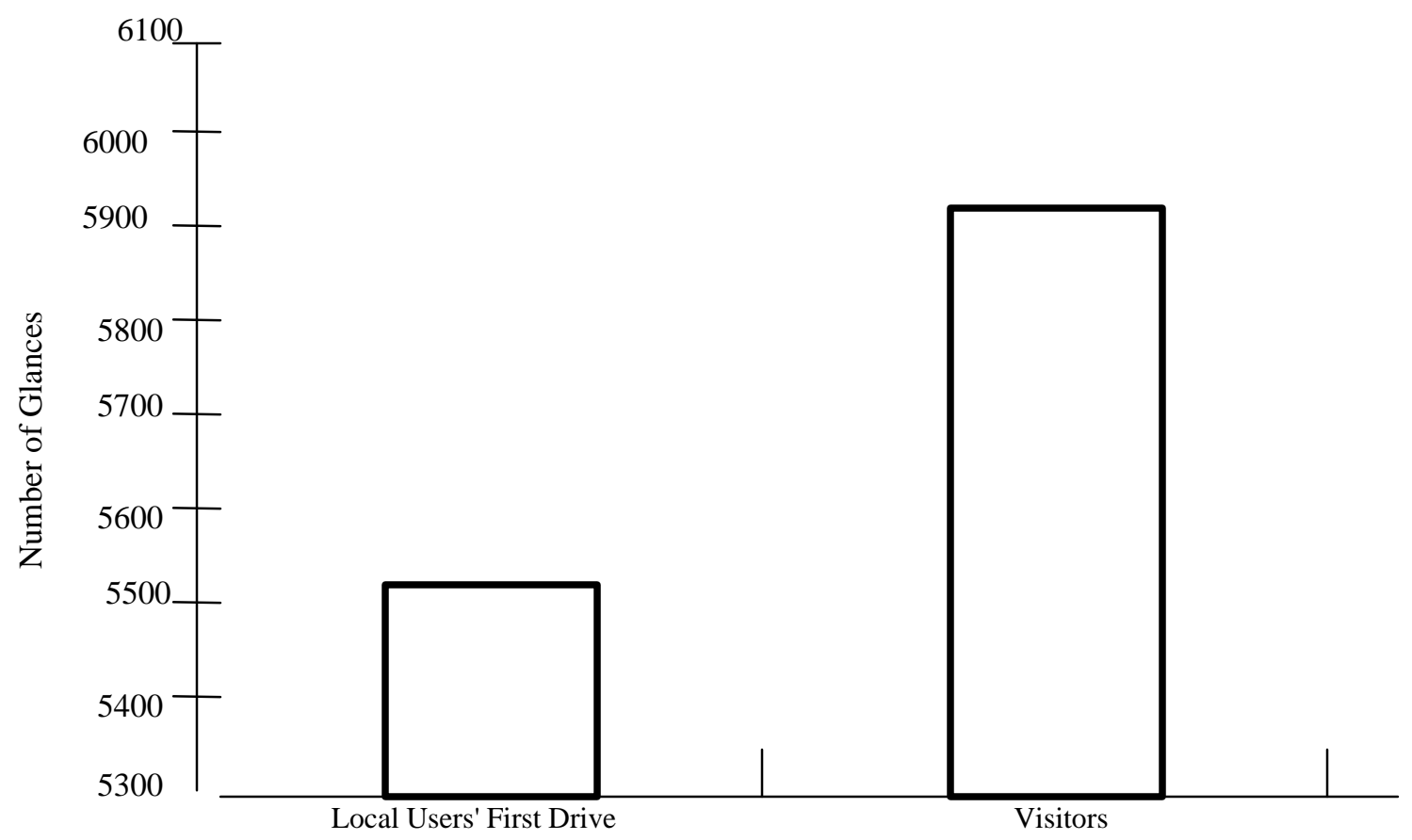

Figure 121. Number of glances to the navigation display for drivers of differing area familiarity.

Figure 122 shows that visitors also had a higher number of glances to roadway-relatedfeatures when compared to the local users. These results show that visitors had shorter glances to more locations than the local users, presumably because they were less familiawith the Orlando area.

A breakdown of the duration and number of glances to the navigation aids by visitor/localuser and navigation condition appear in figures 123 and 124. Note that the visitors hadlonger and fewer glances to the route map without voice condition. It is possible that the visitors found this display more difficult to use due to the overall high workload required by navigating in an unfamiliar location with a navigation aid that also demanded high workload.

\section{Variance in Lateral Acceleration}

As shown in figure 125, the novice local users had a significantly higher variance in lateralccelerations than the visitors. This measure is probably indicative of driver "caution" or"driving aggressiveness,"as opposed to driving performance per se. 


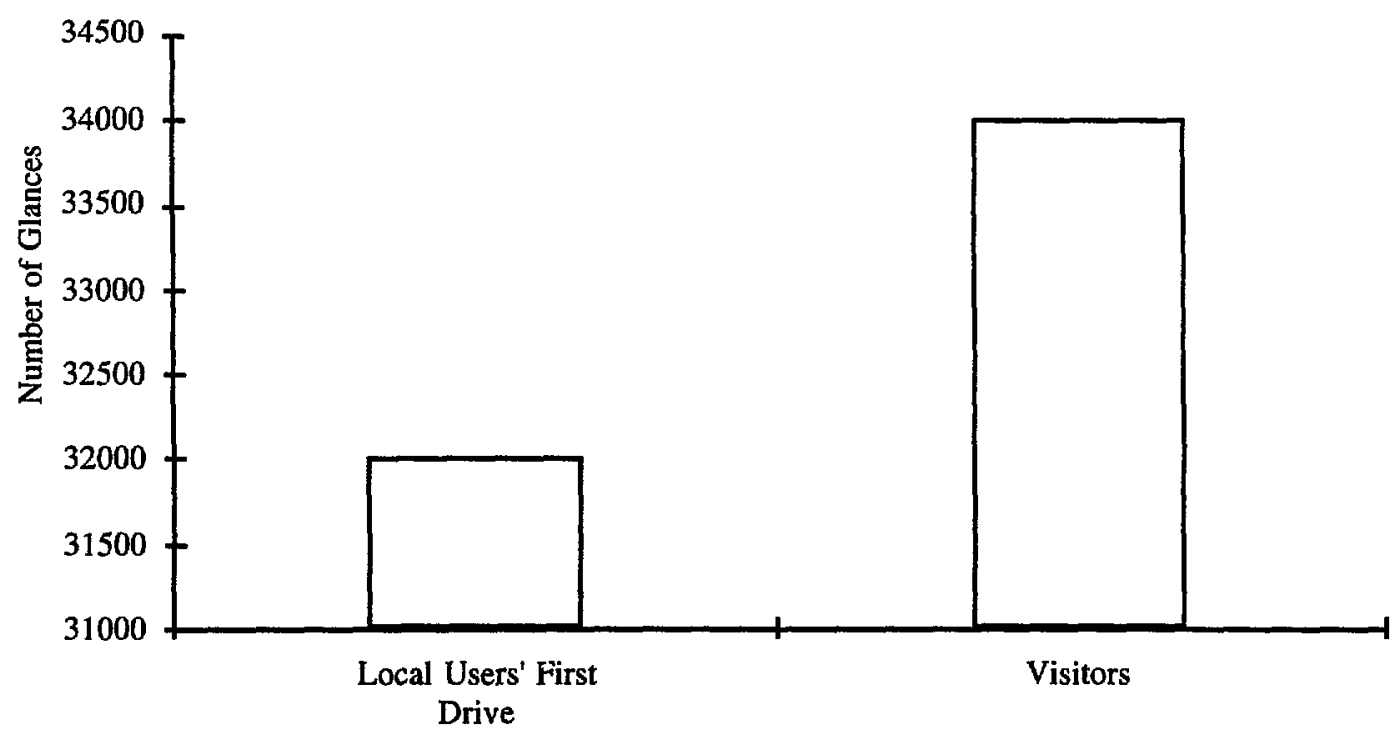

Figure 122. Number of glances to the roadway for drivers of differing area familiarity.

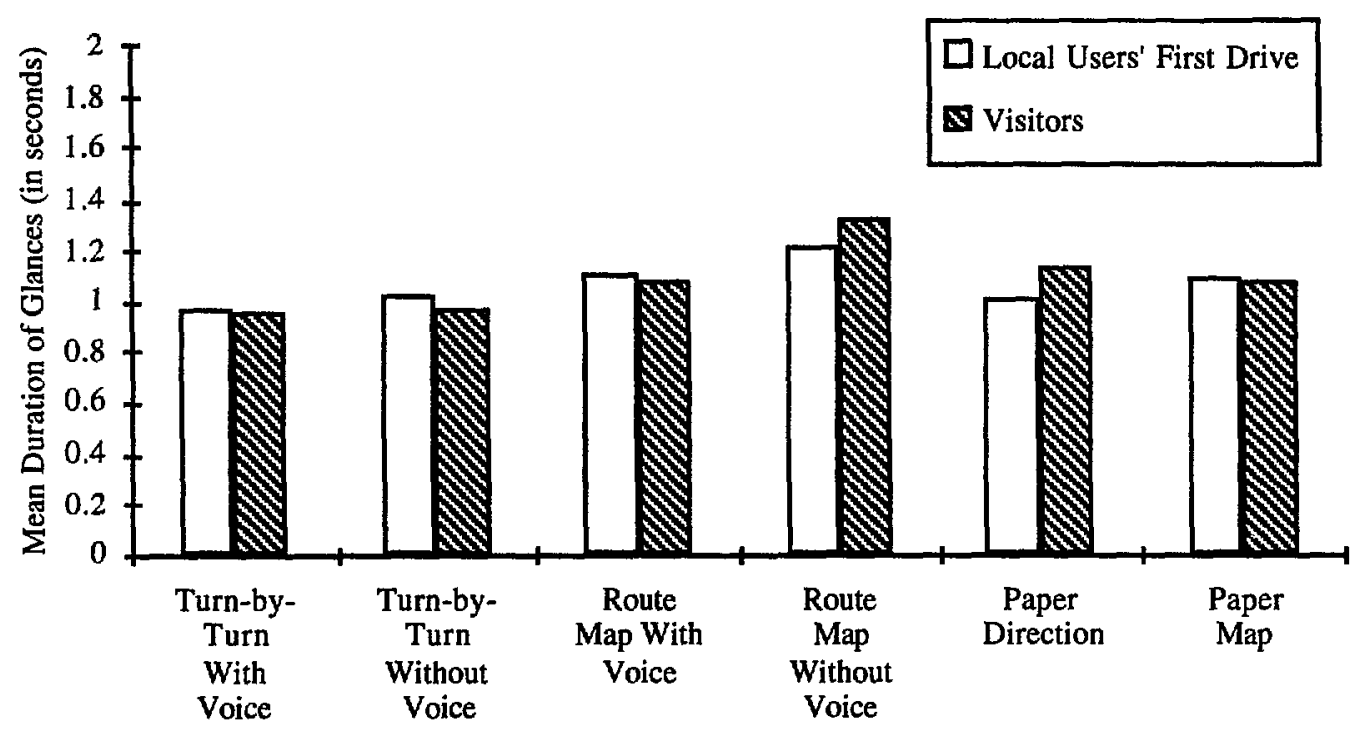

Figure 123. Duration of glances to the navigation display for each navigation condition compared across drivers of differing area familiarity.

\section{Performance Measure Discussion}

Although the results were sparse in comparing the driving performance between novice local users and visitors, they were somewhat surprising. It was hypothesized that because novice local users were more familiar with the driving area, they would have better or less erratic driving performance than the visitors. In actuality, the opposite appeared to be true. The driving performance measures showed that the visitors drove more cautiously than the novice local users. 


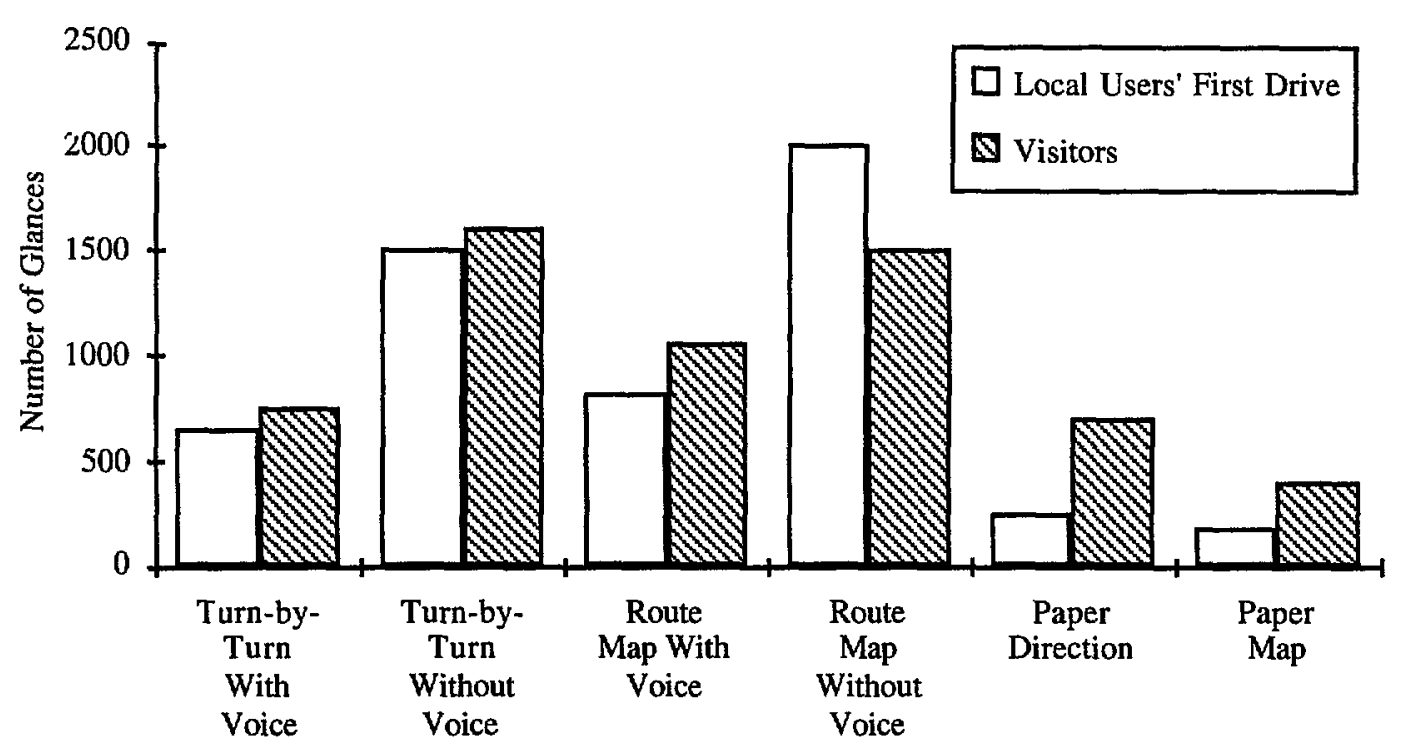

Figure 124. Number of glances to navigation display for each navigation condition compared across drivers of differing area familiarity.

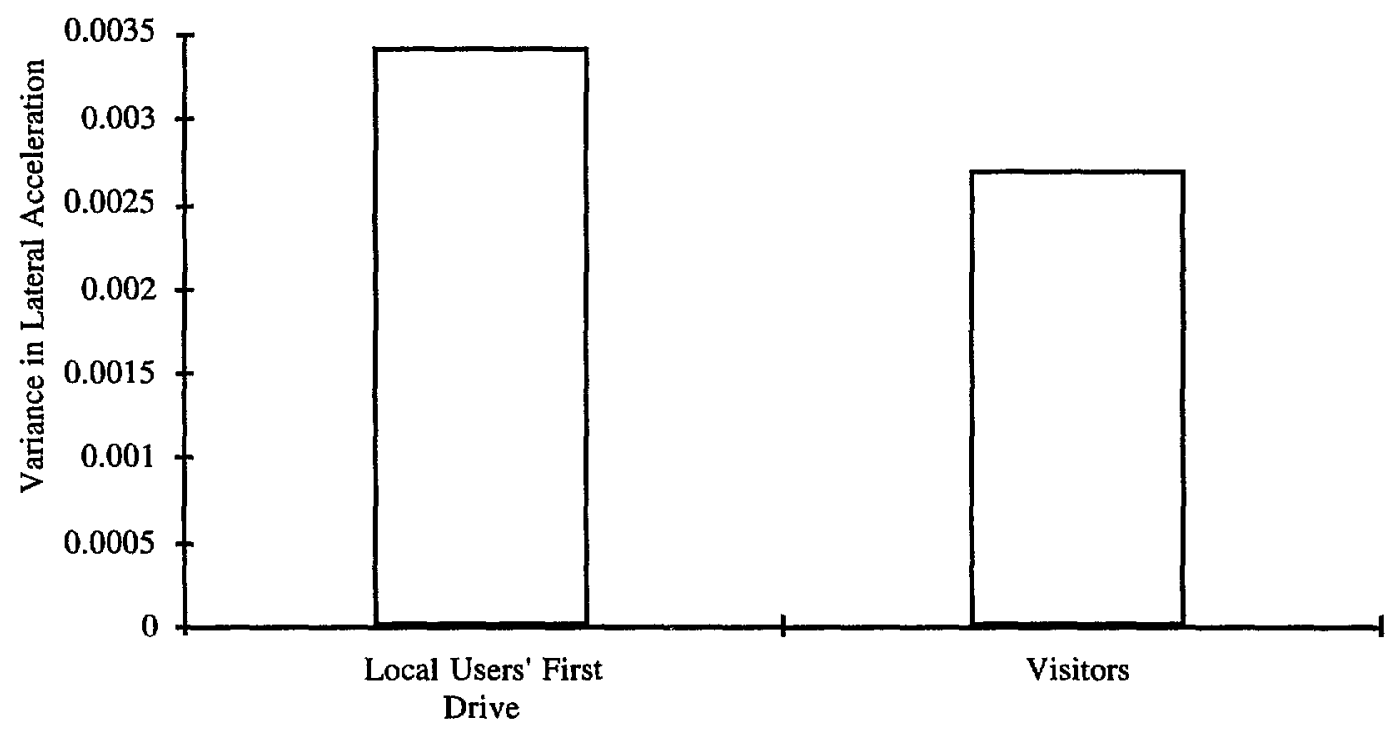

Figure 125. Variance in lateral acceleration for drivers of differing area familiarity.

There was a difference between the two area familiarity groups' number of glances to the navigation aids. Novice local users had substantially fewer glances of approximately the same duration. When considering that novice local users had less visual attention demand to the navigation aids overall, these results together with the driving performance results support the theory that the local users drove less cautiously than the visitors. 


\section{Measures of Performance for Navigation Performance}

The driver navigation performance ANOVA's for area familiarity by age are shown in table 23 and for familiarity by navigation condition in table 24 . As shown in the tables, no driver type main effect was significant for any of the dependent variables for either analysis. In one case, the time that was required to plan a route - an area familiarity by navigation condition interaction - was significant at the $\mathrm{p}<0.05$ level.

Table 23. ANOVA's for age and drivers of differing area familiarity.

\section{Time Required to Plan a Route}

Source

Local users' first drive \& Visitors

Age group

Local users' first drive \& Visitors X Age group

Error

\begin{tabular}{|l|r|r|r|r|}
\hline & \multicolumn{1}{|c|}{$\mathrm{dF}$} & \multicolumn{1}{|c|}{ MS } & \multicolumn{1}{c|}{$\mathrm{F}$} & \multicolumn{1}{c|}{$\mathrm{P}$} \\
\hline & 1 & 12670.888 & 3.33 & 0.0837 \\
\hline & 1 & 313719.696 & 10.19 & 0.0048 \\
\hline & 1 & 133839.193 & 4.35 & 0.0508 \\
\hline
\end{tabular}

Time Required to Drive to a Destination

Source

Local users' first drive \& Visitors

Age group

Local users' first drive \& Visitors X Age group

Error

$\mathrm{dF}$

\begin{tabular}{r|r|r|r|}
\multicolumn{1}{|c|}{ MF } & M & P \\
\hline 1 & 20862.4368 & 0.11 & 0.7460 \\
\hline 1 & 441870.183 & 2.29 & 0.1469 \\
\hline 1 & 121980.477 & 0.63 & 0.4366 \\
\hline 19 & 193155.72 & \multicolumn{3}{|l}{} \\
\hline
\end{tabular}

Time Required to Plan and Drive to a Destination

Source

Local users' first drive \& Visitors

Age group

Local users' first drive \& Visitors X Age group

Error

$\mathrm{dF}$

\begin{tabular}{|r|r|r|r|r|}
\hline \multicolumn{1}{|c|}{$\mathrm{dF}$} & \multicolumn{1}{c|}{ MS } & \multicolumn{1}{c|}{$\mathrm{F}$} & $\mathrm{P}$ \\
\hline 1 & 30953.3429 & 0.15 & 0.7011 \\
\hline 1 & 1500354.89 & 7.36 & 0.0138 \\
\hline 1 & 511434.911 & 2.51 & 0.1294 \\
\hline 19 & 203878.60 & \multicolumn{3}{|c|}{} \\
\hline
\end{tabular}

Number of Stops During Drive to a Destination

Source

Local users' first drive \& Visitors

Age group

Local users' first drive \& Visitors X Age group

Error

\section{Mean Duration of Stops}

\begin{tabular}{|l|r|r|r|r|}
\hline Source & \multicolumn{1}{|c|}{ MF } & \multicolumn{1}{|c|}{ MS } & \multicolumn{1}{c|}{ P } \\
\hline Local users' first drive \& Visitors & 1 & 2964.03710 & 0.80 & 0.7771 \\
\hline Age group & 1 & 53634.5041 & 1.49 & 0.2364 \\
\hline Local users' first drive \& Visitors X Age group & 1 & 3629.85169 & 0.10 & 0.7541 \\
\hline Error & 20 & 35996.591 & \multicolumn{3}{|l}{} \\
\hline
\end{tabular}


Table 24. ANOVA's for navigation condition and drivers of differing area familiarity.

\begin{tabular}{|c|c|c|c|c|}
\hline \multicolumn{5}{|l|}{ Time Required to Plan a Trip } \\
\hline Source & $\mathrm{dF}$ & MS & $\mathrm{F}$ & $\mathrm{P}$ \\
\hline Local users' first drive \& Visitors & 1 & 104613.54 & 2.04 & 0.1678 \\
\hline Navigation condition & 5 & 1853171.74 & 70.39 & 0.0001 \\
\hline Navigation condition X Local users' first drive \& Visitors & 5 & 107847.81 & 4.10 & 0.0020 \\
\hline Subjects (Local users' first drive \& Visitors) & 21 & 51263.72 & & \\
\hline $\begin{array}{l}\text { Navigation condition X Subjects (Local users' first drive \& } \\
\text { Visitors }\end{array}$ & 101 & 26327.28 & & \\
\hline \multicolumn{5}{|l|}{ Time Required to Drive to a Destination } \\
\hline Source & $\mathrm{dF}$ & MS & $\mathrm{F}$ & $\bar{P}$ \\
\hline Local users' first drive \& Visitors & 1 & 21829.63 & 0.11 & 0.7469 \\
\hline Navigation condition & 5 & 329840.96 & 3.83 & 0.0032 \\
\hline Navigation condition X Local users' first drive \& Visitors & 5 & 77462.38 & 0.90 & 0.4839 \\
\hline Subjects (Local users' first drive \& Visitors) & 21 & 204158.82 & & \\
\hline $\begin{array}{l}\text { Navigation condition X Subjects (Local users' first drive \& } \\
\text { Visitors }\end{array}$ & 101 & 86017.83 & & \\
\hline \multicolumn{5}{|l|}{ Time Required to Plan and Drive to a Destination } \\
\hline Source & $\mathrm{dF}$ & MS & $\mathrm{F}$ & $\mathrm{P}$ \\
\hline Local users' first drive \& Visitors & 1 & 30850.47 & 0.11 & 0.7458 \\
\hline Navigation condition & 5 & 3423852.40 & 29.85 & 0.0001 \\
\hline Navigation condition X Local users' first drive \& Visitors & 5 & 170534.42 & 1.49 & 0.2008 \\
\hline Subjects (Local users' first drive \& Visitors) & 21 & 285940.90 & & \\
\hline $\begin{array}{l}\text { Navigation condition X Subjects (Local users' first drive \& } \\
\text { Visitors }\end{array}$ & 101 & 114714.30 & & \\
\hline \multicolumn{5}{|l|}{ Number of Stops During Drive to a Destination } \\
\hline Source & $\mathrm{dF}$ & MS & $\mathrm{F}$ & $\mathrm{P}$ \\
\hline Local users' first drive \& Visitors & 1 & 8.63272095 & 0.19 & 0.6664 \\
\hline Navigation condition & 5 & 78.010019 & 3.96 & 0.0025 \\
\hline Navigation condition X Local users' first drive \& Visitors & 5 & 11.7312512 & 0.59 & 0.7039 \\
\hline Subjects (Local users' first drive \& Visitors) & 22 & 45.22412 & & \\
\hline $\begin{array}{l}\text { Navigation condition X Subjects (Local users' first drive \& } \\
\text { Visitors }\end{array}$ & 104 & 19.71898 & & \\
\hline
\end{tabular}


Table 24. ANOVA's for navigation condition and drivers of differing area familiarity (continued).

\begin{tabular}{|c|c|c|c|c|}
\hline Source & $\underline{\mathrm{df}}$ & $\underline{\mathrm{MS}}$ & $\underline{F}$ & $\underline{\mathbf{P}}$ \\
\hline Local users' first drive \& Visitors & 1 & 4616.83985 & 0.12 & 0.7279 \\
\hline Navigation condition & 5 & 127024.766 & 8.11 & 0.0001 \\
\hline $\begin{array}{l}\text { Navigation condition x Local users } \\
\text { first drive \& Visitors }\end{array}$ & 5 & 22408.473 & 1.43 & 0.2192 \\
\hline $\begin{array}{l}\text { Subjects (Local users' first drive \& } \\
\text { Visitors) }\end{array}$ & 22 & 37176.74 & & \\
\hline $\begin{array}{l}\text { Navigation condition x Subjects } \\
\text { (Local users' first drive \& Visitors) }\end{array}$ & 104 & 15656.66 & & \\
\hline
\end{tabular}

Time Required to Plan a Trip.

Figure 126 shows the interaction for the amount of time required to plan a trip. As can be seen, the largest difference appears for the paper map control condition. There appears to be very little difference for the route map configurations, as well as the turn-by-turn without voice condition. The turn-by-turn with voice display reveals that visitors took somewhat longer to plan their trip than the local users' did on their first drive. The difference results are probably indicative of area familiarity.

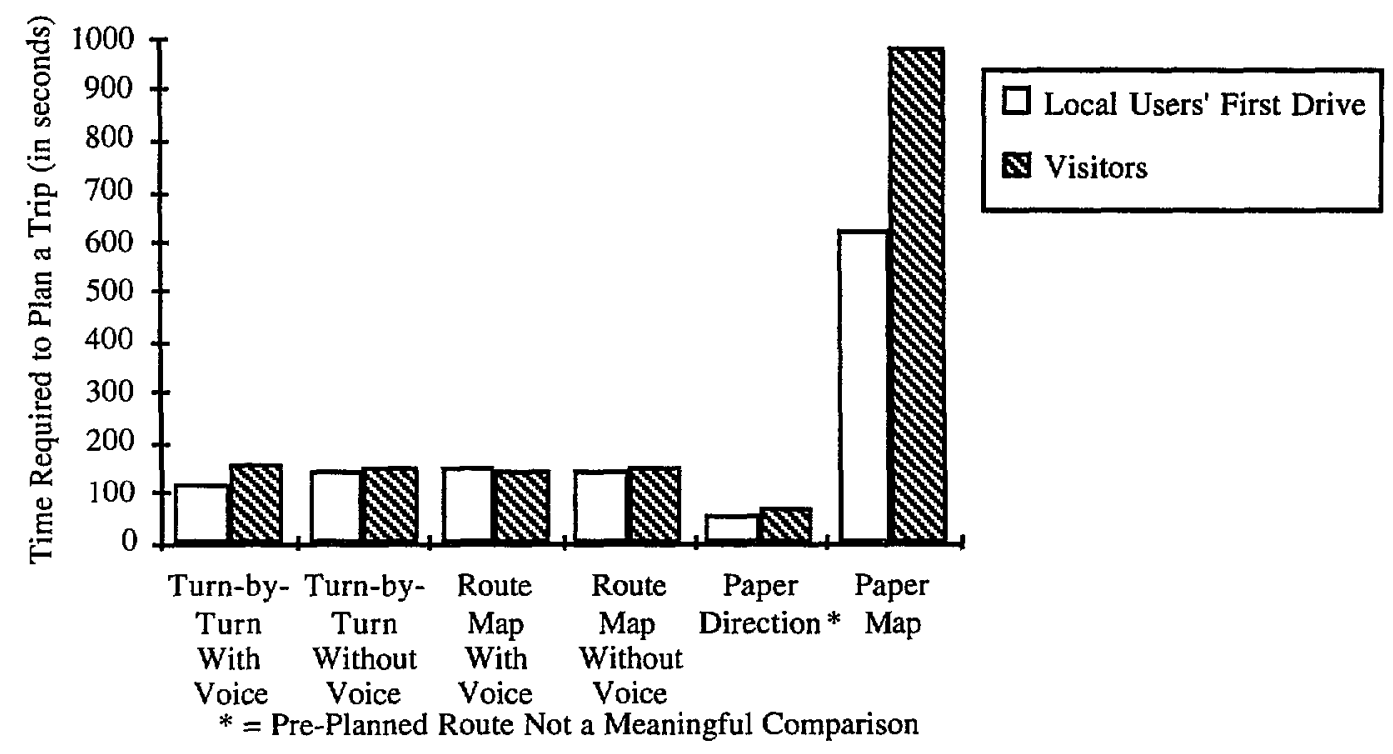

Figure 126. Amount of time required to plan a trip shown for each navigation condition compared across drivers of differing area familiarity.

\section{Number of Navigation-Related Errors.}

Figure 127 shows the number of navigation-related errors broken down by visitor and local user first drive. Overall, the number of errors is equivalent. Surprisingly, the local users were lost and off route a greater number of time than the visitors. This difference may have been due to local user anticipation of the route due to area familiarity. That is, local users 
paid less attention to the details of the route since they generally knew where they were going. This hypothesis is supported by the navigation-aid eye glance data.

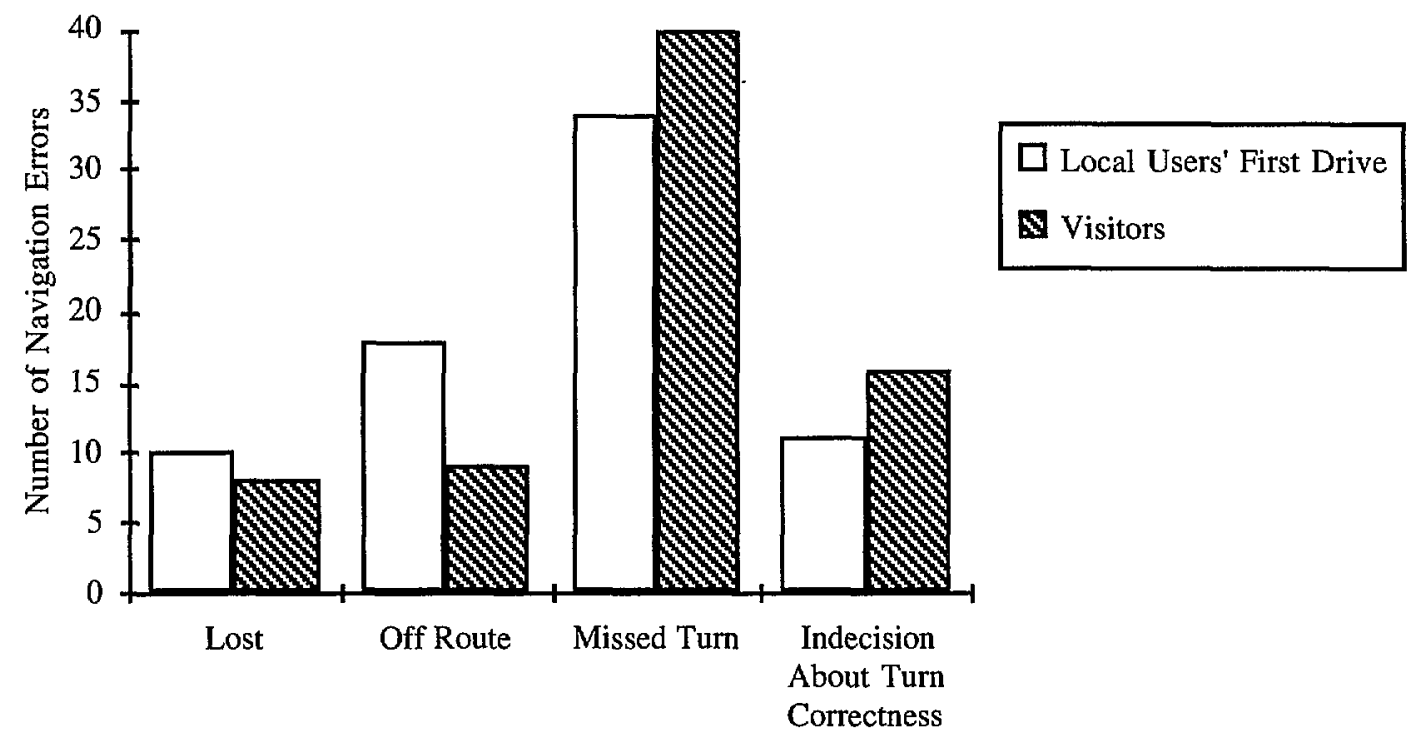

Figure 127. Number of navigation-related errors by type compared across drivers of differing area familiarity.

The results for the number of navigation-related incidents by each navigation condition reveal that, overall, visitors and novice local users' had the same number of errors (see figures 128 and 129). When the incidents are broken down by type, the results show that visitors had a higher number of missed turns, and indecision about turn correctness for turn-by-turn with voice. Neither group got lost using this condition. For the turn-by-turn without voice navigation condition, novice local users had three incidents of being off route; whereas the visitors had none. Visitors were more indecisive about turn correctness than novice local users. The number of incidents for route map with voice revealed novice local users had more missed turns, less times off route, and less indecision about turn correctness. Visitors also got lost three times, while novice local users did not get lost at all. For the navigation condition of route map without voice, novice local users had one more missed turn than did the visitors. The paper directions navigation control condition revealed that visitors had more missed turns. The paper map control conditions found that visitors had a higher number of missed turns than local users' first drive, but they had fewer times off route and being lost.

\section{Discussion For Navigation Performance}

There were no main effect differences found for driver type area familiarity navigation performance. Drivers of both groups took roughly the same amount of time to plan and to drive to a destination. There was a significant difference for the interaction between driver type and navigation condition, which indicates that visitors had more difficulty using the paper map, although this result is not supported by the other navigation performance measures. One theory might be that visitors had a harder time orienting themselves using the map than did novice local users. Novice local users may have known which streets ran north, south, east or west or local landmarks to help in using the map or guiding them to the destination. 


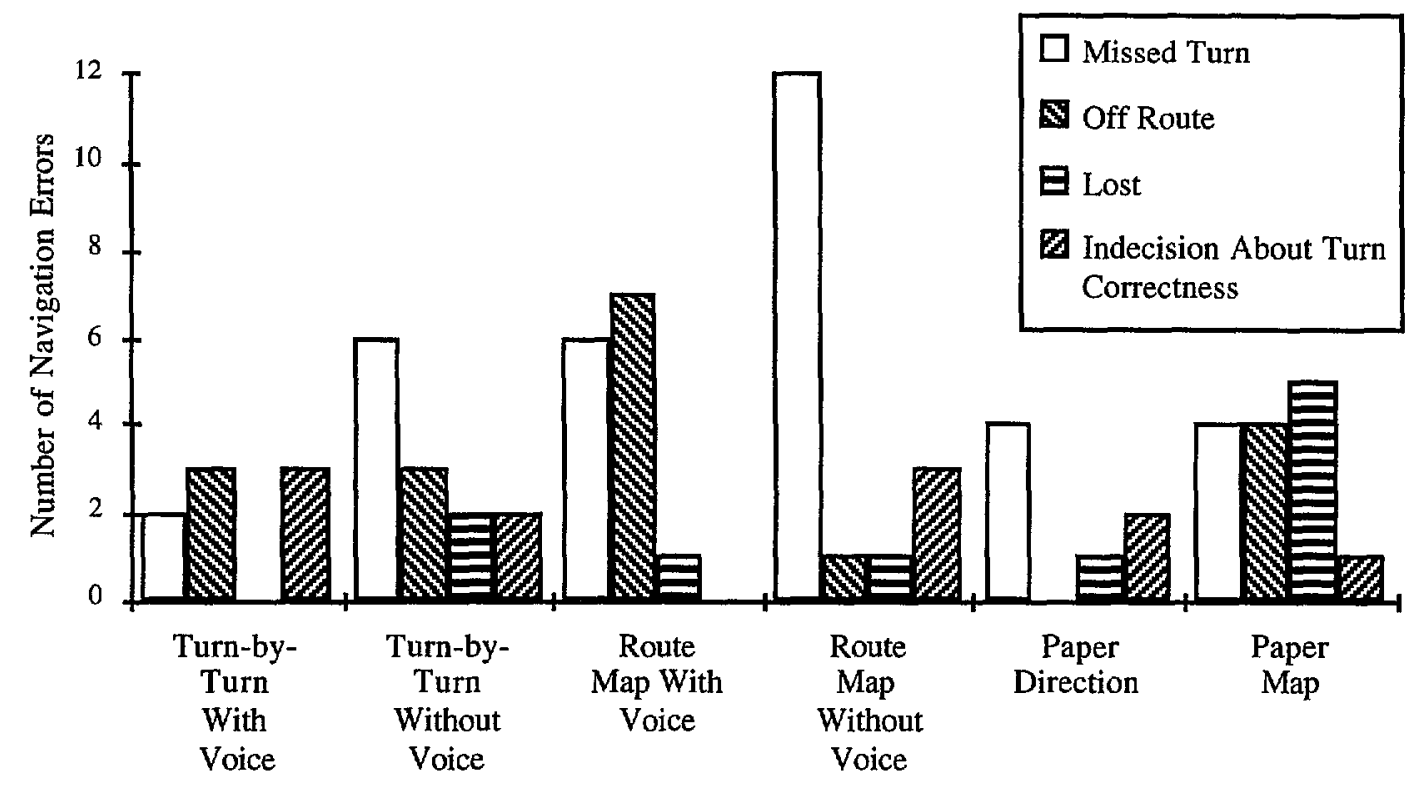

Figure 128. Number of navigation-related errors by type committed on local users' first drive, compared across each navigation condition.

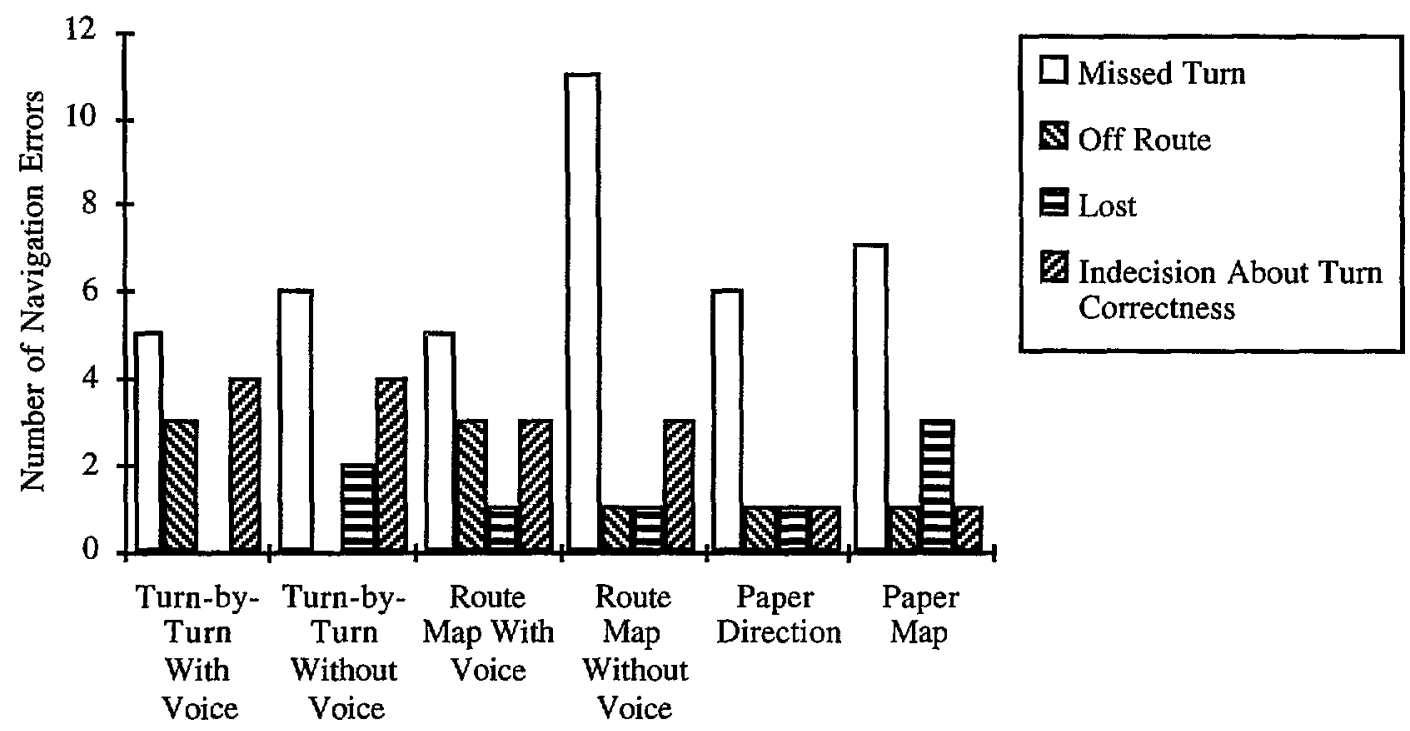

Figure 129. Number of navigation-related errors by type committed by visitors, compared across each navigation condition.

It is interesting to note that the local users were lost and off-route more than the visitors, which might indicate that they were relying on a greater number of external cues and landmarks to navigate. This is consistent with the eye glance findings which indicate that local users looked at the navigation aids less. Perhaps the local users were relying upon this knowledge to take alternative routes not planned by the system. If this was the strategy, the in-vehicle experimenter was unaware of it. 


\section{Additional Measures of Performance Indicative of Ease of Use}

The ANOVA tables for the subjective measure of mental workload are shown in tables 25 and 26. As shown, no differences in workload were present for area familiarity at the $\mathrm{p}<0.05$ level.

Table 25. Workload ANOVA's for area familiarity by age.

\section{Time Stress}

Source

Local users' first drive \& Visitors

Age group

Local users' first drive \& Visitors X Age group

Error

\begin{tabular}{|l|l}
\hline & $\mathrm{dF}$ \\
\hline & \\
\hline & \\
\hline
\end{tabular}

\begin{tabular}{|r|r|r|r|} 
& \multicolumn{1}{|c|}{ MS } & $\mathrm{F}$ & $\mathrm{P}$ \\
\hline 1 & 1.63922425 & 0.59 & 0.4531 \\
\hline 1 & 0.11896685 & 0.04 & 0.8388 \\
\hline 1 & 0.13504572 & 0.05 & 0.8284 \\
\hline 20 & 2.7991109 & \multicolumn{3}{|c|}{} \\
\hline
\end{tabular}

Visual Workload

Source

Local users' first drive \& Visitors

Age group

Local users' first drive \& Visitors X Age group

Error

Psychological Workload

Source

Local users' first drive \& Visitors

Age group

Local users' first drive \& Visitors X Age group

Error

$\mathrm{dF}$

$\mathrm{dF}$

\begin{tabular}{|r|r|r|l|} 
& \multicolumn{1}{|c|}{ MS } & F & P \\
\hline 1 & 11.6756527 & 1.94 & 0.1786 \\
\hline 1 & 0.30465835 & 0.05 & 0.8241 \\
\hline 1 & 0.26163258 & 0.04 & 0.8368 \\
\hline 20 & 6.009040 & & \\
\hline
\end{tabular}

\section{Combined Workload}

\begin{tabular}{|c|c|c|c|c|}
\hline Source & $\mathrm{dF}$ & MS & $\mathrm{F}$ & $\mathrm{P}$ \\
\hline Local users' first drive \& Visitors X Age group & 1 & 0.01748958 & 0.00 & 0.9461 \\
\hline
\end{tabular}

\section{Measures of Performance for Near Misses and Unsafe Acts}

\section{Total Number Of Safety-Related Errors.}

Figure 130 shows the total number of safety-related errors for area familiarity. Local users of both age groups had more safety-related errors than visitors. 
Table 24. ANOVA's for navigation condition and drivers of differing area familiarity.

Time Required to Plan a Trip

Source

Local users' first drive \& Visitors

Navigation condition

Local users' first drive \& Visitors X Navigation condition

Subjects (Local users' first drive \& Visitors)

Navigation condition X Subjects (Local users' first drive \&

Visitors

Time Required to Drive to a Destination

Source

Local users' first drive \& Visitors

Navigation condition

Local users' first drive \& Visitors X Navigation condition

Subjects (Local users' first drive \& Visitors)

Navigation condition X Subjects (Local users' first drive \&

Visitors

$\mathrm{dF}$

\begin{tabular}{|c|c|c|c|}
\hline & MS & $\mathrm{F}$ & $\mathrm{P}$ \\
\hline 1 & 6.1105 & 1.76 & 0.1978 \\
\hline 5 & 3.2084 & 6.40 & 0.0001 \\
\hline 5 & 0.8959 & 1.79 & 0.1212 \\
\hline 22 & 3.4646 & & \\
\hline 109 & 0.5009 & & \\
\hline
\end{tabular}

Time Required to Plan and Drive to a Destination

Source

Local users' first drive \& Visitors

Navigation condition

Local users' first drive \& Visitors X Navigation condition

Subjects (Local users' first drive \& Visitors)

Navigation condition X Subjects (Local users' first drive \&

Visitors

Number of Stops During Drive to a Destination

Source

Local users' first drive \& Visitors

Navigation condition

Navigation condition X Local users' first drive \& Visitors

Subjects (Local users' first drive \& Visitors)

Navigation condition X Subjects (Local users' first drive \&

Visitors
$\mathrm{dF}$

109

2.6387

0.4707

\begin{tabular}{|r|r|r|r|r|}
\hline & $\mathrm{dF}$ & \multicolumn{1}{|c|}{ MS } & $\mathrm{F}$ & $\mathrm{P}$ \\
\hline & 1 & 1.6476 & 0.62 & 0.4379 \\
\hline & 5 & 2.2727 & 4.83 & 0.0005 \\
\hline & 5 & 0.9695 & 2.06 & 0.0760 \\
\hline 22 & 2.6387 & \multicolumn{3}{|c|}{} \\
\hline & 109 & 0.4707 & \multicolumn{3}{|c|}{} \\
\hline
\end{tabular}

\begin{tabular}{|c|c|c|c|c|}
\hline & $\mathrm{dF}$ & MS & $\mathrm{F}$ & $\overline{\mathrm{P}}$ \\
\hline & 1 & 11.8589 & 2.17 & 0.156 \\
\hline & 5 & 5.5063 & 7.83 & 0.0001 \\
\hline dition & 5 & 1.1756 & 1.67 & 0.1476 \\
\hline & 22 & 5.4576 & & \\
\hline Irive \& & 109 & 0.7034 & & \\
\hline
\end{tabular}

(n)

dF 1

\begin{tabular}{r|r|r|l|} 
& \multicolumn{1}{|c|}{ MS } & \multicolumn{1}{|c|}{ F } & P \\
\hline 1 & 7.2609 & 1.45 & 0.2421 \\
\hline 5 & 257180 & 4.07 & 0.0020 \\
\hline 5 & 1.0101 & \multicolumn{3}{|l}{} \\
\hline 109 & 5.0245 & \multicolumn{3}{|l}{} \\
\hline
\end{tabular}




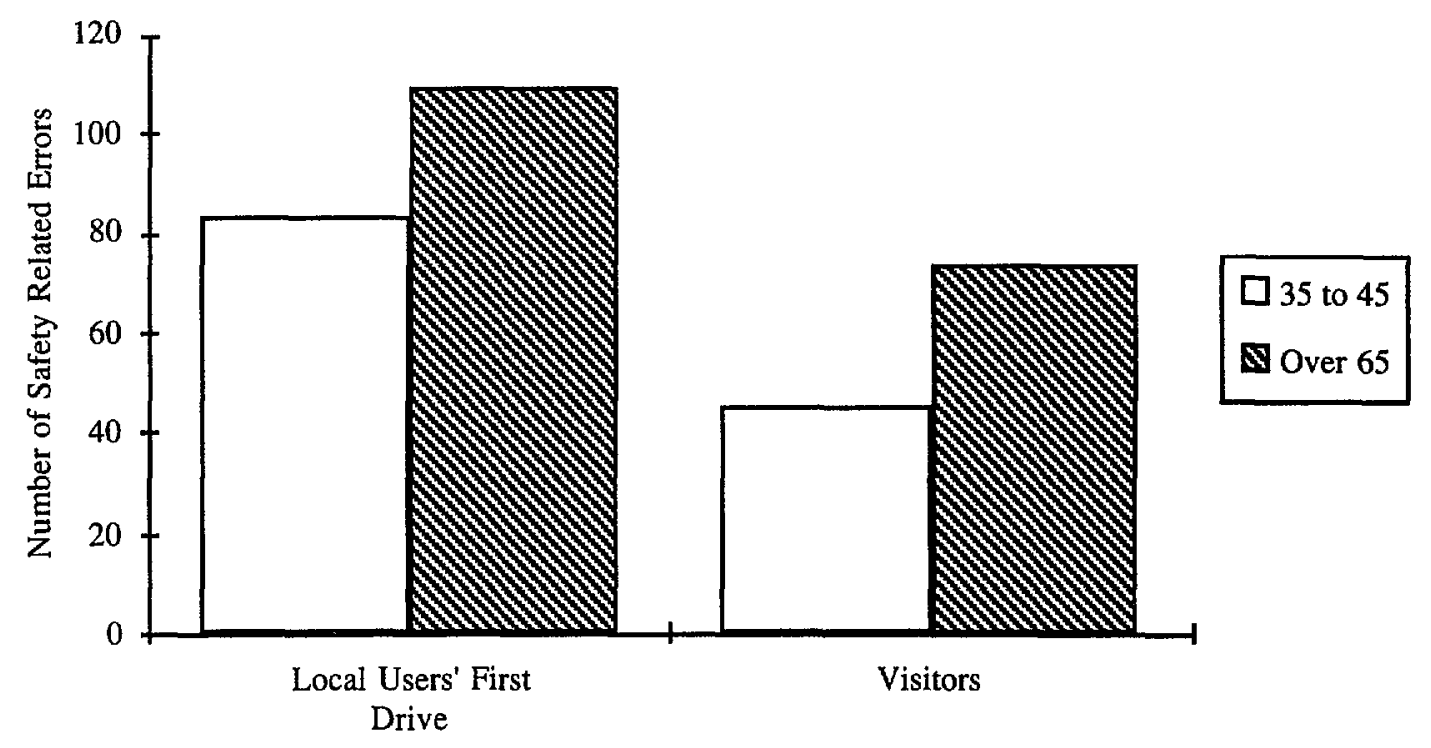

Figure 130. Total number of safety-related errors compared across drivers of differing area familiarity and age.

Number and Duration of Lane Excursions.

Figure 131 shows the number of lane deviations broken out by driver type and age. The local users had a greater number of lane deviations as did the older age group. However, even though the older drivers drove more cautiously in terms of speed than younger drivers, they still had more lane deviations. Overall, the visitors had fewer lane deviations, presumably due to more cautious behavior. This hypothesis is also supported by the driving performance measures.

The number of lane deviations for the interaction of display condition by area familiarity is shown in figure 132. Except for route map without voice and paper map, novice local users had more lane deviations than visitors for all navigation conditions. It is interesting to note, that the visitors exhibited a greater number of lane deviations in the conditions which have been shown in other issues sections to have the highest levels of workload.

\section{Discussion of Safety-Related Errors for Area Familiarity}

The total number of safety-related errors and lane deviations for both age groups reveals that novice local users had more errors than visitors. These finding are counter-intuitive given that the visitors had more navigation aid glances than the local users. This supports the previously discussed theory that in general, novice local users drove less cautiously than the visitors.

\section{General Discussion of Area Familiarity}

Navigation performance results showed that despite the fact that visitors made more and longer glances to the navigation conditions, they were lost or off route less than local users. Visitors took longer to plan a route to a destination. This was especially true for the paper map control condition. It is apparent that the local users felt they knew where they were 
going in general; whereas visitors were less sure of the area and planned in more detail and followed navigation aid directions more carefully.

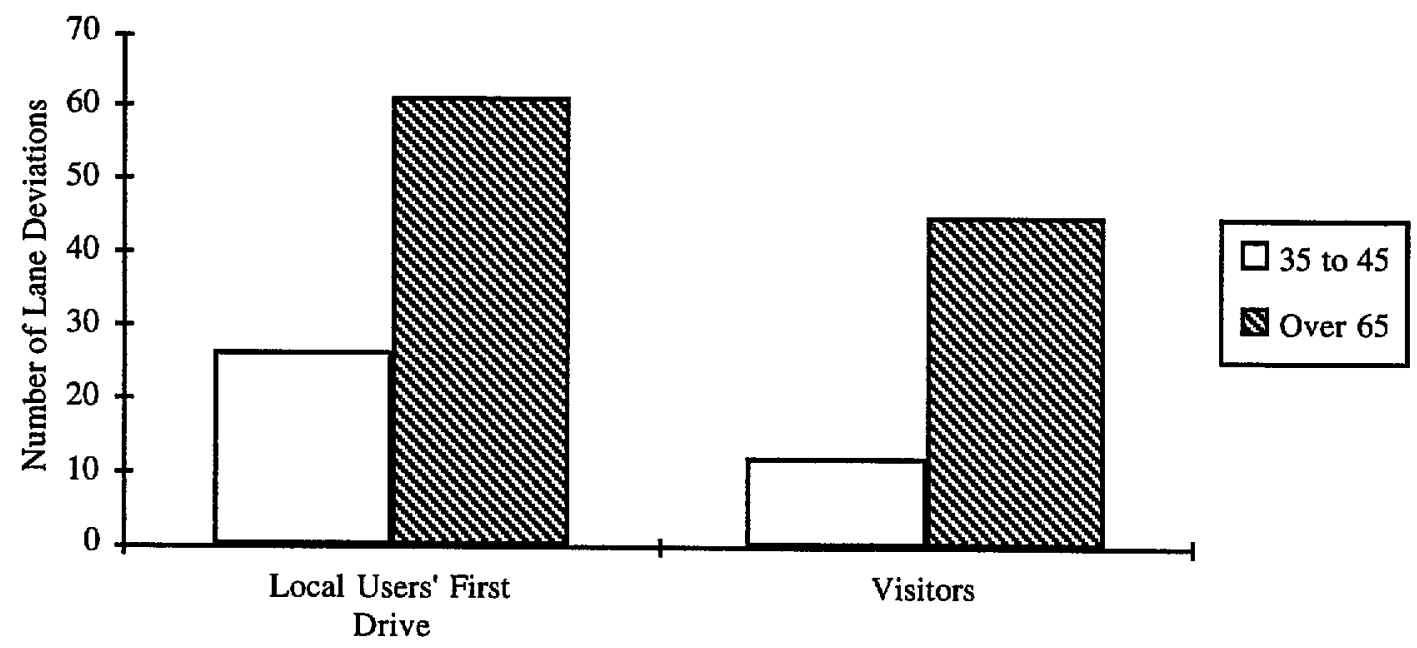

Figure 131. Number of lane deviations compared across drivers of differing area familiarity and age.

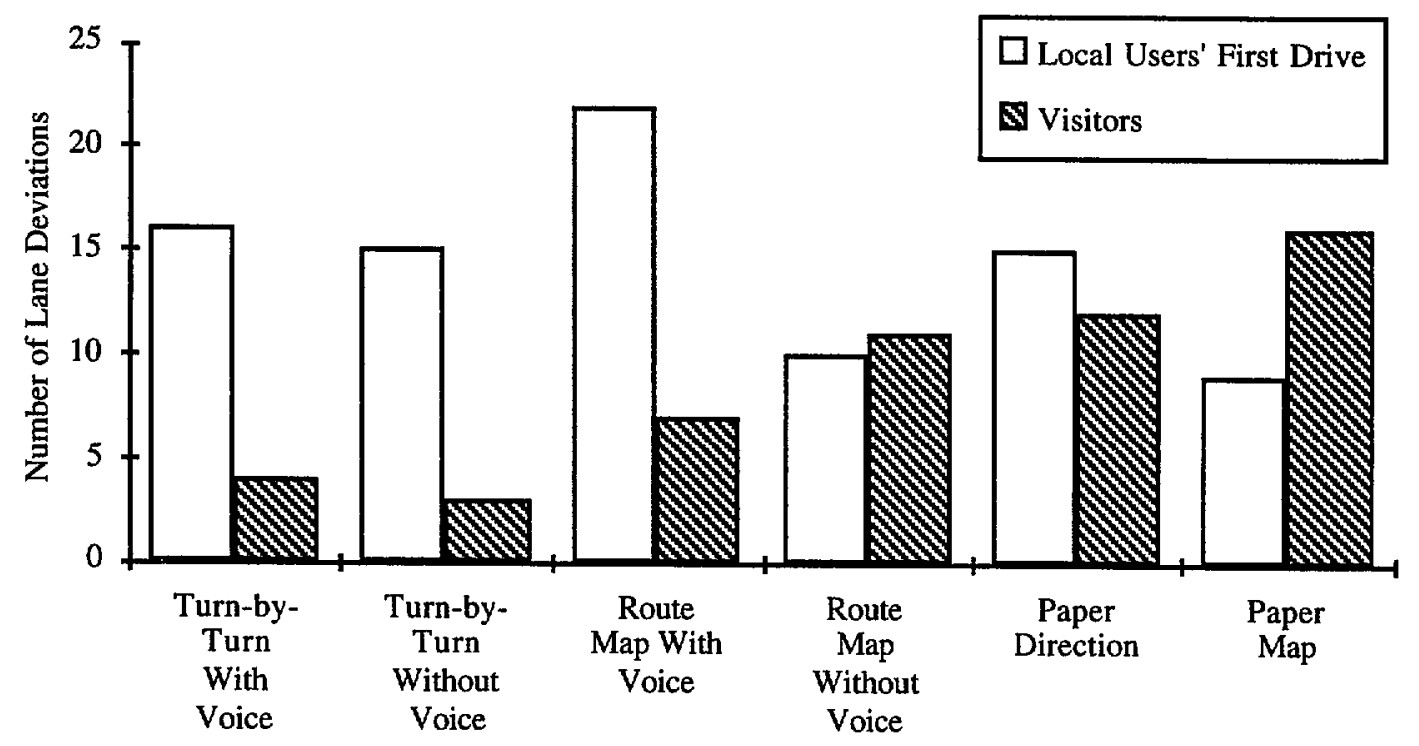

Figure 132. Number of lane deviations for each navigation condition compared across drivers of differing area familiarity and age.

Overall, the safety findings reveal that local users drove less cautiously than the visitors. This hypothesis is supported by findings that local users looked at, and depended less upon, the navigation aids but had poorer driving performance while using the system. Local users had a higher lateral acceleration variance and more lane deviations than visitors. 
Local users may have exercised less caution in their driving behavior because they were more familiar with the area and, therefore, more confident while driving.

These results indicate that the visitors were more cautious in route planning, following, and driving than the local users. 